Western University

Scholarship@Western

Digitized Theses

Digitized Special Collections

1967

\title{
The Photochemical Beckmann Rearrangement
}

Takao Tabata

Follow this and additional works at: https://ir.lib.uwo.ca/digitizedtheses

\section{Recommended Citation}

Tabata, Takao, "The Photochemical Beckmann Rearrangement" (1967). Digitized Theses. 365.

https://ir.lib.uwo.ca/digitizedtheses/365

This Dissertation is brought to you for free and open access by the Digitized Special Collections at Scholarship@Western. It has been accepted for inclusion in Digitized Theses by an authorized administrator of Scholarship@Western. For more information, please contact tadam@uwo.ca,

wlswadmin@uwo.ca. 
The author of this thesis has granted The University of Western Ontario a non-exclusive license to reproduce and distribute copies of this thesis to users of Western Libraries. Copyright remains with the author.

Electronic theses and dissertations available in The University of Western Ontario's institutional repository (Scholarship@Western) are solely for the purpose of private study and research. They may not be copied or reproduced, except as permitted by copyright laws, without written authority of the copyright owner. Any commercial use or publication is strictly prohibited.

The original copyright license attesting to these terms and signed by the author of this thesis may be found in the original print version of the thesis, held by Western Libraries.

The thesis approval page signed by the examining committee may also be found in the original print version of the thesis held in Western Libraries.

Please contact Western Libraries for further information:

E-mail: libadmin@uwo.ca

Telephone: (519) 661-2111 Ext. 84796

Web site: http://www.lib.uwo.ca/ 


\author{
by \\ Takao Tabata \\ Department of Chem1stry
}

Submitted in partial fulfillment

of the requirements for the degree of

Doctor of Phllosophy

Faculty of Graduate Studies

The University of Western Ontario

London, Canada

May, 1967

(C) Takao Tabata 1967 


\section{ABSTRACT}

The investication was directed towards elucidating the mechanism of the Photochemical Beckmann Rearrangement of benzaldoximes to the respective amides. Intramolecularity of the reaction was established by conducting a cross-over experiment in the presence of labelled benzaldoxime $18_{0}$ and p-tolualdoxime. Furthermore, spectroscopic and chemical evidence for the intermediacy of 3-p-anisyloxazirane upon irradiation of p-anisaldoxime is presented.

In this photochemical transformation, the necessity of a protonic source for a facile reaction of benzaldoxime to benzamide was demonstrated by irradiating the oxime at $290 \mathrm{~m} \mu$ in cyclohexane. The quantum efficiency in the presence of acetic acid was considerably greater than in its absence. It was further established that triplet sensitized reaction at $334 \mathrm{~m} \mu$, using triphenylene, led to benzamide with a very low quantum yield. Without acetic acid, no amide was formed and only geometrical isomerization was observed. Spectroscopic evidence for the occurrence of a triplet energy transfer from efficient triplet donors (benzophenone, triphenylene, and biacetyl) to benzaldoxime in both rigid and fluid media is also given. Quantum yields of geometrical isomerization in both directions were determined and it was found that the geometrical isomerization was much more efficient than the 
structural isomerization. The photostationary states (PSS) were also determined by direct and sensitized irradiations. On the basis of the results, the states in which structural and geometrical isomerizations occurred were assigned as singlet and triplet, respectively. A triplet-triplet annihilation process is postulated to account for the isolation of benzamide under triplet-sensitized reaction. The triplet energy level of benzaldoxime is estimated to be between 49 and $62 \mathrm{k} . \mathrm{cal}$. The effect of radiant energy on the quantum yield of the rearrangement was as also examined. Of the three wavelengths used, 210, 253.7 and $290 \mathrm{mp}$, the efficiency is highest at 290mp. Close scrutiny of the ultraviolet spectrum of benzaldoxime indicated that the initial excitation is of a $\pi x^{*}$ type rather than of a $m^{*}$ type. The 0-0 singlet level is assigned to the absorption at $291.5 \mathrm{~m} \mu$ in cyclohexane for ofbenzaldoxime.

The effects of substituents, solvents, acids and oxygen on the rearrangement were also investigated at $290 \mathrm{~m} \mu$. The highest quantum yield of the benzaldoximes substituted with para groups $\left(\mathrm{CH}_{3} \mathrm{O}, \mathrm{CH}_{3}, \mathrm{H}, \mathrm{F}, \mathrm{Cl}\right.$, and $\left.\mathrm{Br}\right)$ was observed for the unsubstituted oxime. No correlation of the quantum yields of benzamides with the Hammett sigma parameter was obtained. Furthermore, no correlation of the quantum efficiency with Reichardt's solvent-ionizing parameter was observed. The reaction was also found to be sensitive to the concentration as well as to the nature of the acetic acids. Oxygen, 
v

rather than quenching the geometrical 18omerization was found to quench the rearrangement. The effects of substituents, solvents, acids and oxygen are adduced by considering their influence in both the excited singlet state of the benzaldoxime and in the electronic ground state of the intermediates, 3-phengloxaziranes. On the basis of the results so far accumulated, a possible mechanism of the Photochemical Beckmann Rearrangement is proposed. 


\section{ACKNOWLEDGEMENT T}

The author would like to express his appreciation to Professor P. de Mayo for suggesting this problem and for his advice, encouragement, and guidance during the course of this research.

The author wishes to thank Dr. D. Samuel of the Welzman Institute of science for his advice on the synthesis of 1sotopically labelled compounds, Professor E. Schmitz of Deutsche Akademle der Wissenschaften zu Berlin for making avallable the procedure for the synthesis of 3-phenyloxazirane, and Dr. D.R. Arnold of Union Carblde, Tarrytown, USA for furnishing the data on the triplet energy of various ketones. The technical advice of Dr. R.W. Y1p of the National Research Council of Canada is also recognized.

His appreciation is extended to Mr. R. Zirk for his assistance in measuring the ultraviolet spectra, to $\mathrm{Mr}$. J. Charlton for taking the flash photolysis spectra and to $\mathrm{Mr}$. J. Wasson, Miss. J. O'Dell, and Mr. G. Jenkinson for reading parts of the manuscript. Special thanks are also extended to Mr. C. Halls and. Mrs. K. MacPherson for reading the manuscript and to the Iibrary staffs of the University of Western Ontario Science Ilbrary for their co-operation. 
The support of the National Research Counc1l of Canada throingh grants to Professor de Mayo and through the Ontario Greduate Fellowship to the author is gratefully acknorledged. 
ABSTRACT

ACKNOWLEDGEMEN TS

IIST OF TABLES.

IIST OF FIGURES.

GENERAI INTRODUCTION

IN TRODUC TION

RESULTS AND DISCUSSION.

A. Molecularity of the Photochemical Beckmann Rearrangement.

B. Intervention of an Intermediate............ 36

C. Multiplicity of States.................... 49

I. Quantum Yield of Benzamide by Sensitization 49

II. Quantum Efficiency of Geometrical

Isomerlzation........................

III.Photostationary States or Equilibrium Mixtures by Direct and Sensitized

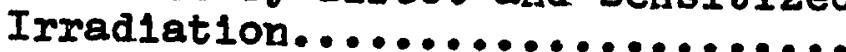

IV. Spectroscopic Evidence fos Triplet Energy Transfer from a Sensitizer to Benzaidoximes 62

v. Effect of Oxygen on the Structural and Geometrical Isomerization of Benzaldoximes. 64

VI. Direct and Sensitized Irradiation........ 74

VII.C1s-Trans Isomerization................ 98

VIII.Quenching by Oxygen................ 110

D. Effects of Wavelengths.................. 117

E. Effects of Substituents, Solvents, and Acids. 132 
I. Effects of Substituents on the Freited Singlet state of Benzaldozimes......... 134

II. Efrects of Solvents and Aclds on the Exclted Singlet State of Benzaldoxime... 155

III. Effects of Substituents, Solvents, and Acids on the Flectronic Ground State of the Intermediate.

F. Summary

EXP ERTMEN TAL

A. Materials.

I. Preparation of Benzaldoximes, p-substituted Benzaldoximes, and p-Methoxyacetophenone Oxime

II. Preparation of Amides. 192

III. Preparation of Aromatic and Ketonic Sensitizers

IV. Purification of Solvents.

V. Organic Acids Selected for Irradiation......... 194

VI. Materials Selected for Phosphorescence Studies

VII. Materials selected for Irradiation of pAnlsaldoxime at Low Temperatures.

B. The Measurements of Quantum Yields.

I. Apparatus Set-Up and I1ght Sources............

II. Cells

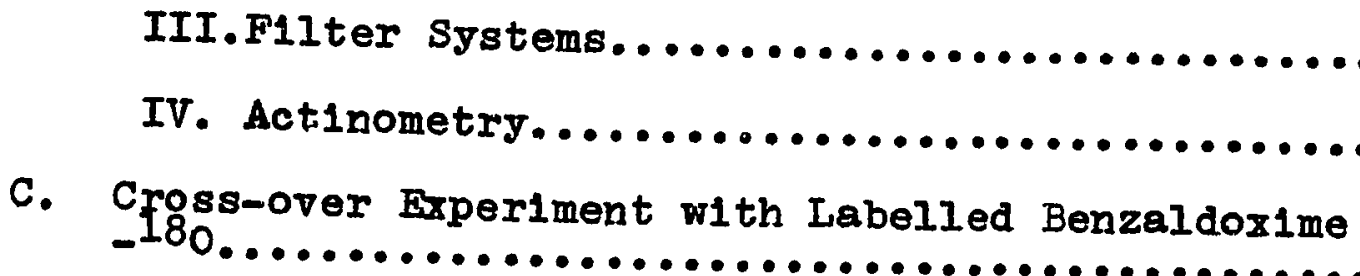

I. Preparation of ${ }^{18} 0$-Labelled Potassium

Hydroxylamine D1 sulfonate.

II. Hydrolja1s of ${ }^{18}$ o-Labelled Potassium

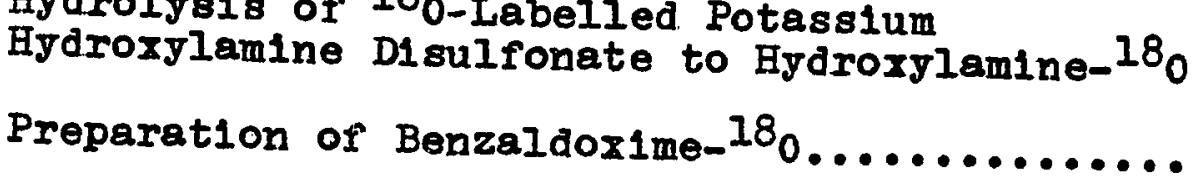


IV. Irradiation of a Mixture of Benzaldoxime- ${ }^{18_{0}}$

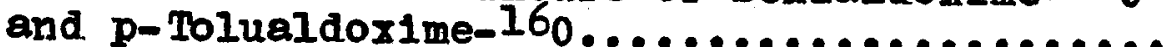

D. Irradiation of $\propto-p-A n 18 a I d o x i m e$ at Low Temperature. 214

I. Optical Absorption Cells................ 214

II. Light Sources........................ 215

III. Irradiation in a Fluid Medium and. Spectrophotometry: General Procedure..............

IV. Irradiation in a Rigid Matrix at $77^{\circ} \mathrm{K}$

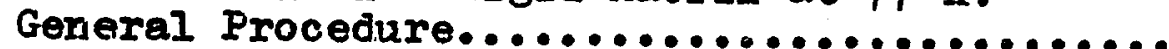

E. Separation of $\alpha$ and $\beta$-Benzaldoximes by Thin Layer Chromatography: Quantitat1ve Analyses..............

I. Method I: Measurement of Spot Area...........

II. Method II: Measurement by Direct U.V. Spectrophotometry...............

III. Extraction of $\alpha$ and $\beta$-Benzaldoximes from the Silica Gel Using a 4:1 Cyolohezane-n-Propanol

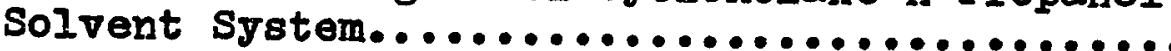

F. Sensitized Irradiations........................ 224

I. Aprotic Reaction Condition in Dioxane........ 224

II. Protonic Reaction Conditions in Cyclohexane.. 227

III. "Prep Scale": Sensitized Reaction of Benzaldoxime under Protonic Reaction Conditions if Cyclohexane...................

G. Quantum Yields of $\alpha$-Benzaldoxime $\left(\phi_{\beta \alpha}\right)$ and $\beta-$

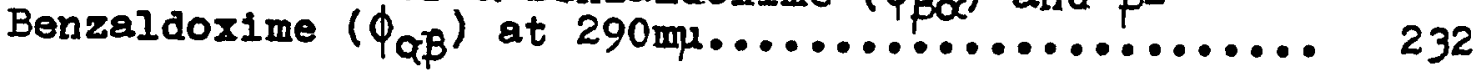

I. In $n-$ Hexane......................... 232

II. In Dioxane.......................... 235

III. In Cyclohexane...................... 236

IV. Analyt1cal Procedures.................. 237

H. Cis-Trans Isomerization: Photostationary States.... 243

I. Direct Irradiation...................... 243

II. Sensitized Irradiation.................. 245 
I. Anission Spectrophotometry: Quenching of Phosphorescence or Sensitizers by of and $\beta$-Benzaldoximes at $770_{\mathrm{K}}$

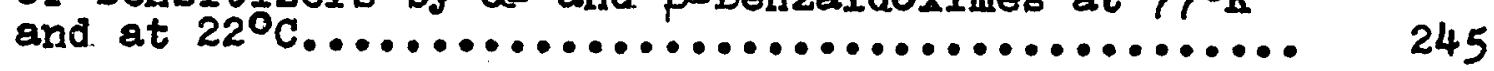

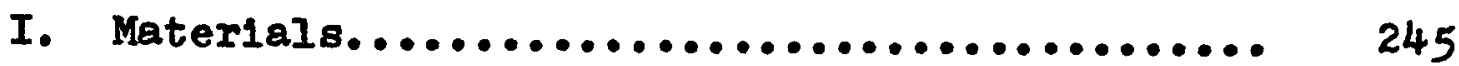

II. Instrumental and Procedure................ 246

J. Quantum Y1eld Measurements of Benzamide at 210mp,

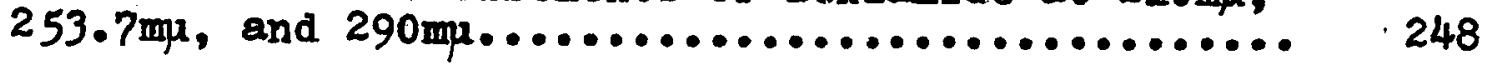

I. Direct Irradiation...................... 248

II. An Estimation of the Quantum Yield of Potassium Ferrioxalate Solution at $210 \mathrm{m \mu} \ldots . . .251$

K. Substituent Effects......................... 257

I. Quantum Yields of p-Substituted Benzamides... 257

II. Infrared Spectrophotometry: Calibration of p-Substituted Benzamides................... 259

III.Large Scale Irradiation of p-Fluorobenzaldoxime 259

IV. Large Scale Irradiation of p-Anisaldoxime.... 260

v. The Rearrengement of p-Methoxyacetophenone

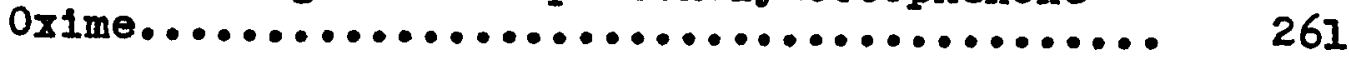

I. Solvent Effects: Quantum Yleld Measurements of Benzamide.................................... 264

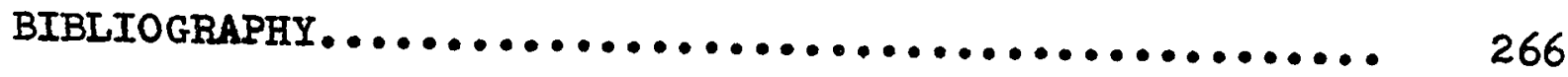

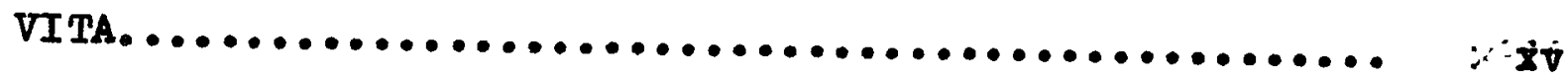




\section{LIST OF TABLES}

I. Irradiation of $\alpha-p-A n i s a l d o x i m e$ in a Rigid Matrid at $77^{\circ} \mathrm{K} . \ldots \ldots \ldots \ldots \ldots \ldots \ldots \ldots \ldots \ldots \ldots \ldots \ldots \ldots \ldots$

II. Quantum Yields of Benzam1de $\left(\phi_{B_{z}-S}\right)_{\lambda_{1}}$ by Sensitization at $290 \mathrm{mp}$ and $334 \mathrm{mp} \ldots \ldots \ldots \ldots \ldots \ldots . . \ldots 5$

III. Effect of Wavelengths on the Bearrangement of Benzaldoxime to Benzamide in Cyclohexane by

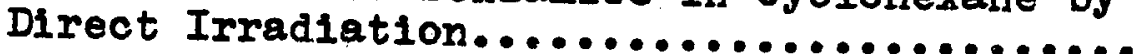

IV. Quantum Yields of Geometrical Isomerization of

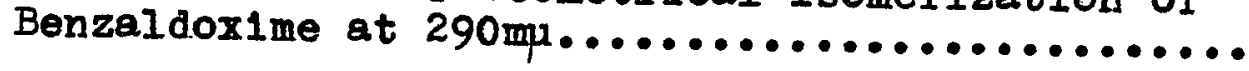

V. Photostationary States (PSS) of of and $\beta$-Benzaldoximes in Dloxane by Direct and Sensitized Irradiation.

VI.

VII.

VIII.

Effect of Oxygen On the Rearrangement of Benzaldoxime to Benzamide in Cyclohexane at $290 \mathrm{mp} . . . . .$. .

Energies of the First Excited Singlet ( SOOO $_{\mathrm{O}}$ ) and the Iriplet $\left(E_{\text {To-o }}\right.$ ) States of Sensitizers........ 78

Ultraviolet Spectral Data of p-Substituted

Benzaldoximes, Benzam1des, and a Formanilide in Cyclohexane.

IX. Brfect of Substituents on the Rearrangement of $p-$ Substituted Benzaldoximes to Amides in the presence of Acetic Acid (0.73M) in Cyclohexane at

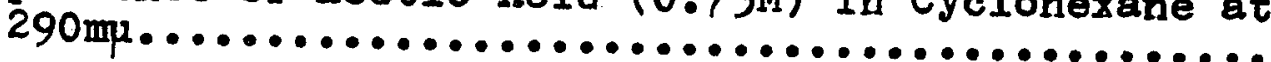

X: Quantum Yields of Rearrangement of p-Methoxyacetophenone ox1me to N-p-An1sylacetamide (I) NMethyi-p-Anisamide (II), and p-Anisamide (III) in Cycloherane at $290 \mathrm{mp} . \ldots \ldots \ldots \ldots \ldots \ldots \ldots \ldots \ldots \ldots . \ldots 140$

XI. Effect of Aprotic Solvents on the Rearrangement of Benzaldoxime to Benzamide at $290 \mathrm{mp}$.............. 164

XII. Effect of Protonic Solvents on the Rearrangement of Benzaldoxime to Benzamide at 290mp........... 165

XIII. Relative Intensity of the Monochromatic Light at $210 \mathrm{mp}$ and $250 \mathrm{mp}$ Measured by a Gelvanometer....... 256

XIV. Quantum Yield of Potassium Ferrloxalate Actinometer Solution at $210 \mathrm{mp} \ldots \ldots \ldots \ldots \ldots \ldots \ldots \ldots 256$ 
Figure

LIST OF FIGURES

1. Schematic ijagram of the Apparatus set up for

Irradiation at Low Temperatures.

2. Low Temperature Irradiation of of-p-Anisaldoxime at $290 \mathrm{mp}$ (Unmixed solution in $n$-hexane)

3. Low Temperature Irradiation of $\alpha$-p-An1saldoxime at $290 \mathrm{mp}$ (Unmixed solution in n-hexane).

4. Low Temperature 0 Irradiation of $\alpha$-p-Anisaldorime at

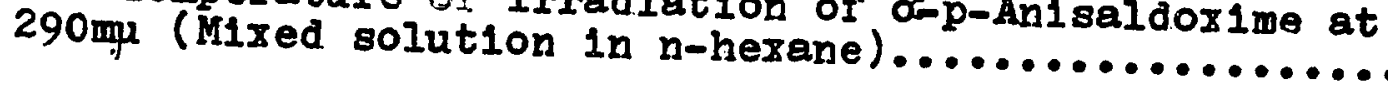

5. Ultraviolet Spectra of $\alpha$ and $\beta$-p-An1saldox1mes, $p-$ Anisamide, N-p-Methoxyformanilide, and 2-Ethyl-3-

6. Photostationary state of Benzaldoxime by Direct Irradiation at $253.7 \mathrm{mp}$ in Dioxane.

7. Photostationary State of Benzaldoxime by Sensitized Irradiation with Triphenylene in Dioxane............ 70 8. Quenching of Phosphorescence of Benzophenone at $77^{\circ} \mathrm{K}$

9. Quenching of Phosphorescence of Triphenglene at $77^{\circ} \mathrm{K}$

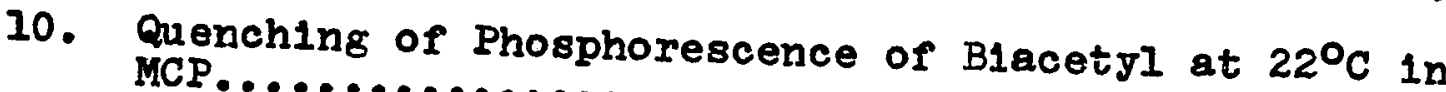
11. Absorption and Emission Spectra of Biacetyl in

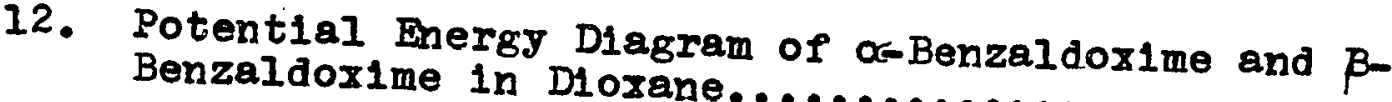

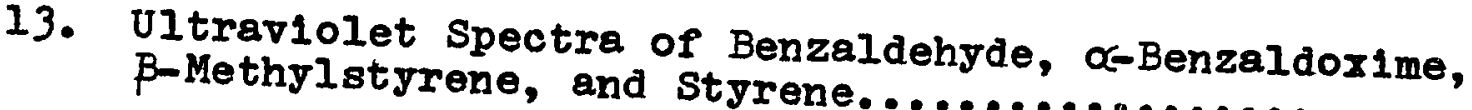

14. Effect of Substituents on the Rearrangement of $p$ Substituted Benzaldoximes to Amldes in the Presence of Acetic Ac1d $(0.73 \mathrm{M})$ in Cyclohexane at $290 \mathrm{mp} \ldots \ldots \ldots$..... 141

15. UItrav1olet Spectra of p-Subst1tuted- $\alpha$-Benzaldoximes

16. Effect of Solvents on the Rearrangement of Benzald-

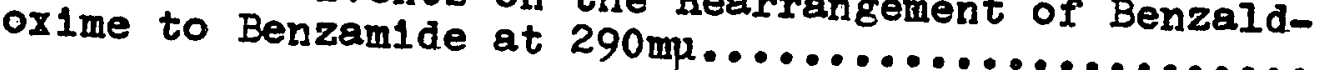


17. Effect of Ac1d (A) on the Rearrangement of Benzaldoxime to Benzamide at $290 \mathrm{mp}$ in Cyclohexane.............. 167

18. Ultraviolet Spectra of o-Benzaldoxime under Varlous Conditions...................................... 168

19. Schemat1c Diagram of the Split-Beam Irradiation Apparatus..................................... 199

20. The Irradiation Block............................... 199

21. The Irradiation Cell used in the Determination of Quantum Yields.................................. 200

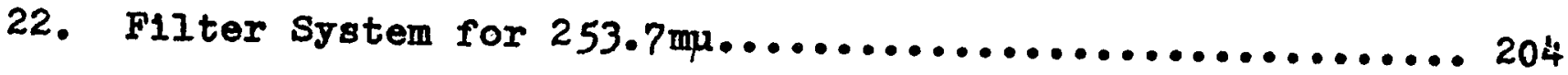

23. Filter System for the Isolation of the $334 \mathrm{mp}$ line from Osram HBO-200 W.Mereury.Lamp........................ 205

24. Calibration Curve of the Ferrioxalate Actinometer Solution............................ Actlnometer 


\section{GENERAL INTRODUCTION}

The reactions investigated include a large variety of processes, some of which are analogous to the more familiar base- and acid-catalyzed reactions. One of the latter is the Beckmann rearrangement. The work described in this thesis has been directed towards the elucidation of the mechanism of the photochemical Beckmann rearrangement of benzaldoximes to their respective amides.

The general topic of photochemical rearrangements of organic molecules in the condensed phase has not been reviewed since $1963(1,2)$ and no general classification of all the reactions has been attempted. For background information, processes related to the present investigation are reviewed. Attention is confined to photochemically induced conversions. involving the cleavage of a group or groups followed by an apparent intramolecular migration. No attempt has been made to include all known photochemical transformations. Rearrangements in which the migration of a group has not been established are excluded (3-6), as are the reactions initiated by cycloaddition $(8,9)$, valence tautomerism $(10)$, and transformation of 2,5-cyclohexadienones (11), santonins $(1,2,12)$, and cyclic enones $(2,13)$.

Those transformations reviewed are arbitrarily 
classified as a) the Photo Fries rearrangement and related reactions, b) Rearrangement of 3-membered ring systems, c) Hotenoatom Reariaingement... A brief review of the normal acid-catalyzed Beckmann rearrangement in the electronic ground state is included to permit a comparison with the photochemical reaction.

a) the Photo Fries Rearrangement and Related Reactions

The general class of photochemical rearrangements represented by Scheme $I$ is now well documented. $Y$ represents the migrating group containing a

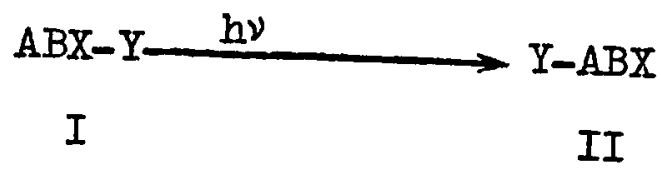

\section{Scheme I}

chromophore capable of undergoing excitation and $A B X$ is a triad which is capable of stabilizing a free radical intermediate by resonance delocalization. $Y$ is known to undergo a 1,3 or a vinylogous 1,5-shift when two termination sites are available. The most common chromophoric group of $Y$ is a carbonyl moiety. Excitation by absorption of light in the adcessible region of the $n \pi *$ electronic transition band, results in an efficient dissociation between a carbonyl group and an adjacent atom by a Norrish Type-I process. (The $n x *$ transition is associated with the promotion of non-bonded electrons in the carbonyl function to a higher $\pi^{*}$ molecular orbital). This type of photochemical cleavage is known for 


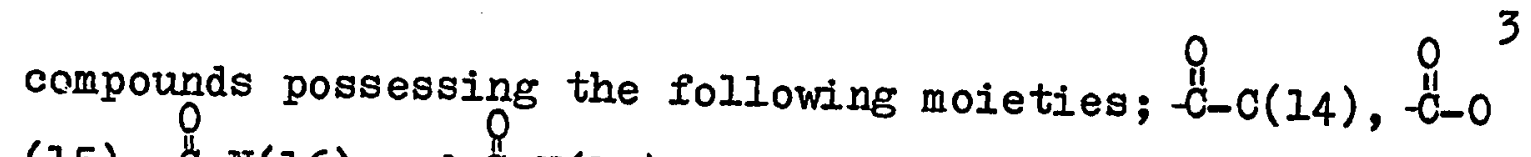
(15), $-\mathbb{C}-N(16)$ and $-S_{-j}-N(18)$. The reaction finds its application in the Photo Fries rearrangement of aryl carboxylates (15) and related reactions of enol esters(20), aryl ethers(19), carboxylic anilides(16) and arenesulphonanilides(18).
\end{abstract}

Apart from the synthetic utility of the Photo Fries reaction (Reaction'), for example, as in the preparation of a key benzophenone intermediate $\underline{2}$ for the synthesis of chlorogreiseofulvin 3 ( $15 \mathrm{e}$ ), attempts have been made to elucidate the mechenism of the reaction represented by scheme $I$, and the system most extensively studied has been that of the aryl esters.

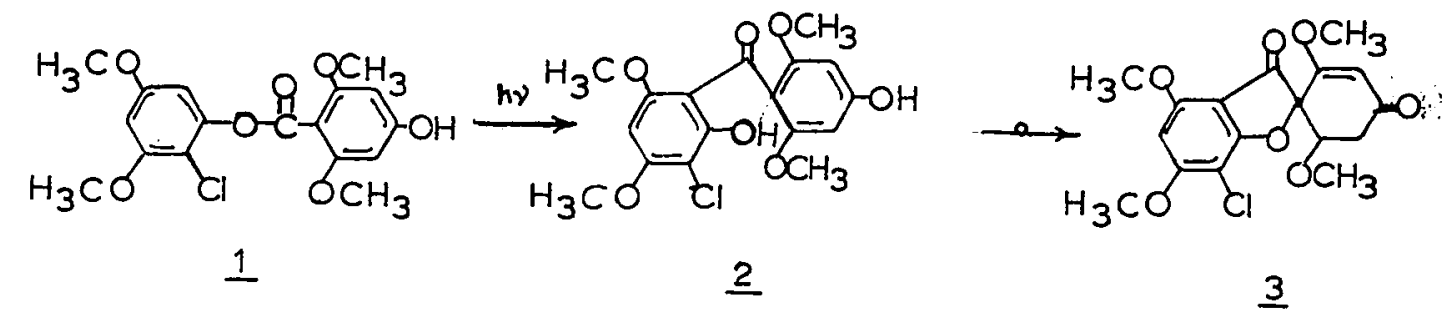

\title{
Reaction I
}

Although no direct eviderse bearing on the photcexcitation of esters is available, and the multiplicity of the states responsible for the rearrangement has not been defined, a mechanism has been proposed for aryl esters which seems generally applicable for system I (Scheme I). From their studies of $p$ t-butylphenylbenzoates, Kobsa (15a) and co-workers postulated that the rearrangement occurred by a facile homolytic cleavage 
of the starting material 4 into a free radical pair $\underline{5}$ followed by a intramolecular recombination within a solvent cage to afford the corresponding ortho hydroxybenzophenone $I$ as represented below in Reaction 2.

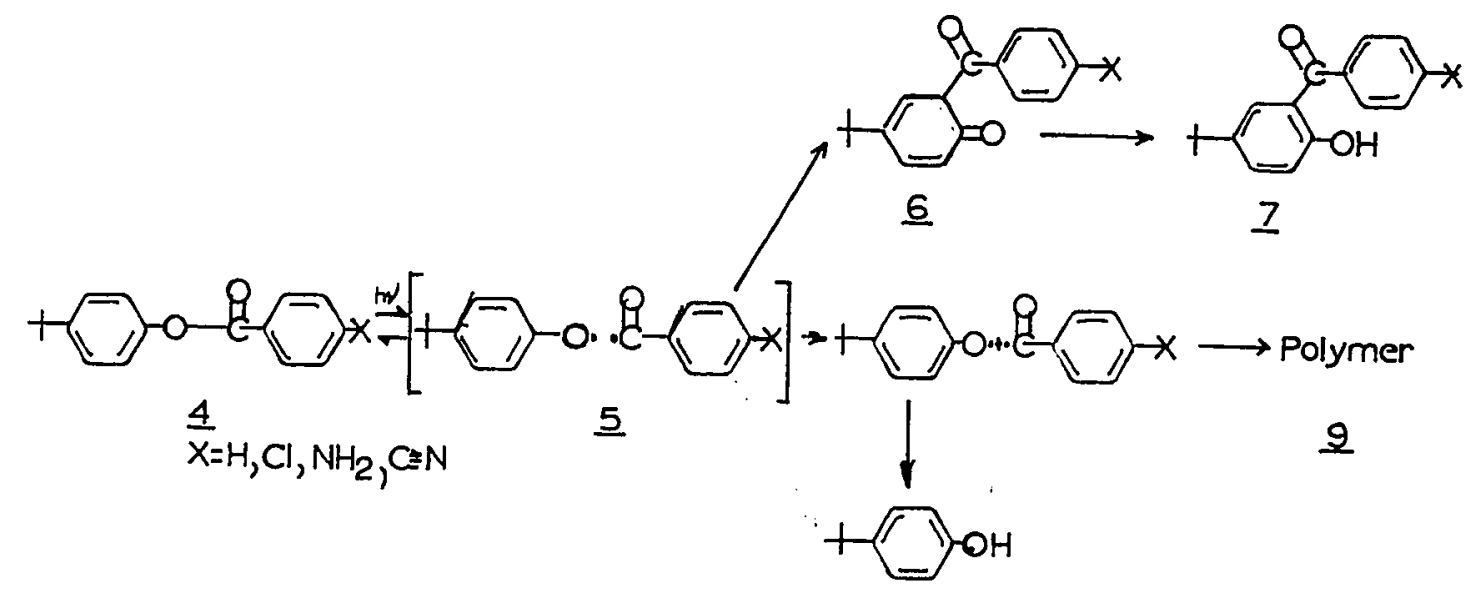

음

Reaction 2

Since Kobsa's first proposal on the mode of rearrangement, results of subsequent investigations by other workers $(18,20)$ tend to support his general scheme. Intramolecularity of the reaction has been established by conducting crossover experiments. An exception, however, has been reported by Elad and co-workers(16a) in the anilide series. Supporting evidence for the intervention of a radical pair intermediate is given by the observation of a slight increase in the quantum efficiency of the reaction when the benzoyl moiety of p-t-butylphenylbenzoate 4 is substituted with an electronwithdrawing group. Stabilization of the migrating acyl portion by an electron-withdrawing group in the transition 
state or the intermediate 5 has been suggested as the main facter responsible for the enhanced quantum efficlency.

Very recently, hovever, Coppinger and Bell (2l) have contested the original proposal of Kobsa from their results for the Photo Fries reaction of phenyl 3,5-di-t-butyl-4hydroxybenzoates. Instead of a free radical type cleavagerecombination mechanism, an intramolecular rearrangement proceeding directly from an exc1ted singlet $\pi \pi^{*}$ state has been suggested.

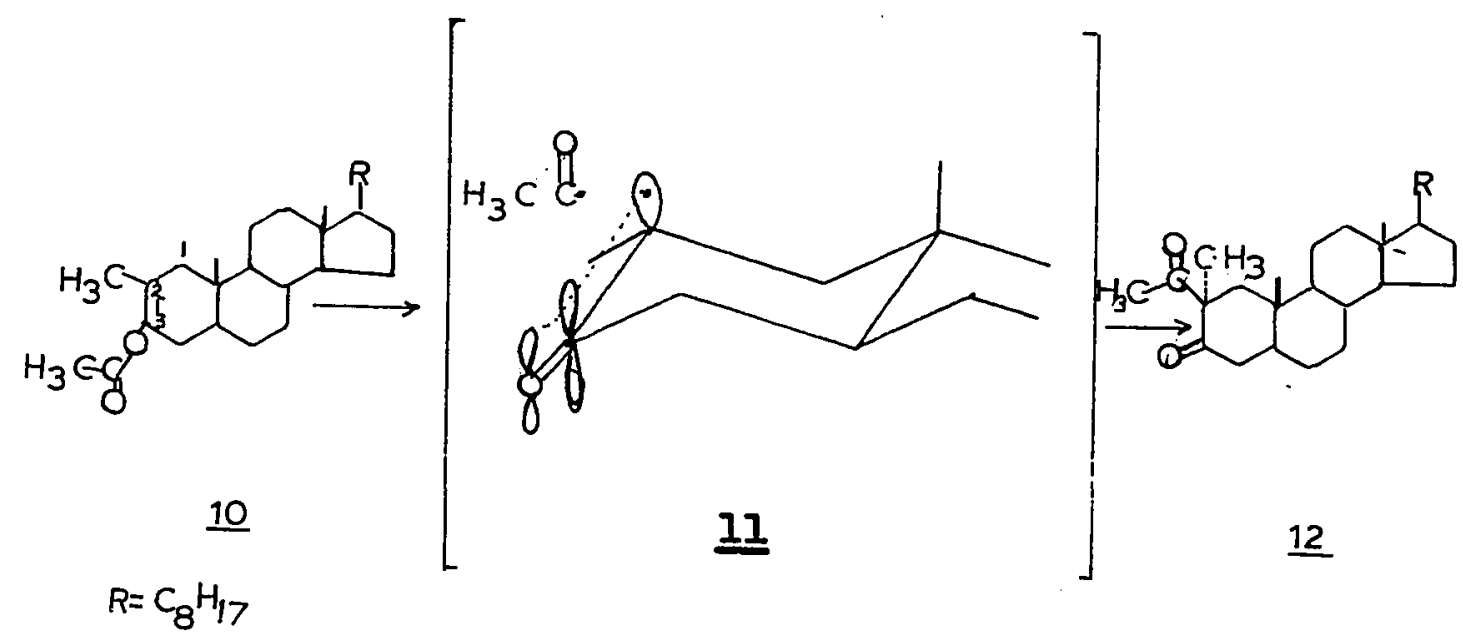

Reaction 3

The reaction, in addition to being intramolecular, seems to be stereoelectronically controlled in one instance. For example, a thermodynamically less stable 1somer, 2- $\alpha$-methyl2-B-aceto-cholestan-3-one 12, was 180lated from the irradiation of 2-methyl-cholest-2-en-3-ol acetate 10. Feldkimel-Gorodetsky and co-workers (20c) have suggested that the orbital occupled by a single electron on $\mathrm{C}_{2}$ could be stabilized by 
the interaction of the exocyclic carbonyl bond if the orbital of $\mathrm{C}_{2}$ were oriented in the axial position, presumably in the intermediate or transition state 11 .

b) the Rearrangement of 3-Membered Ring Systems

Another type of photochemically induced rearrangement is that involving the migration of hydrogen, alkyl, aryl and acyl groups in cyclopropanes and epoxides. For most cases where the reaction has been demonstrated to be of a photochemical nature, the mechanism invoked has been essentially that of a very rapid intramolecular movement of a group via the intervention of a free radical intermediate.

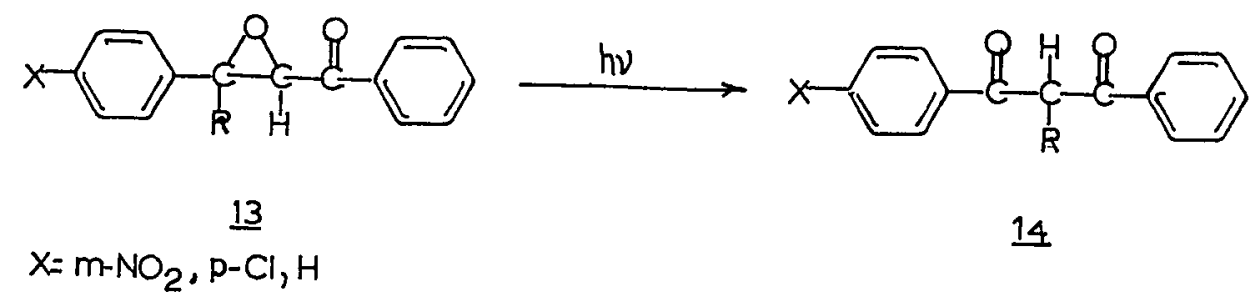

Scheme II

One of the earliest examples was reported in 1918 by Bodforss (25), who discovered the preferential migration of hydrogen over phenyl upon irradiative conversion of 1,3-diphenyl- $\alpha$-epoxyketone $13,(R=X=H)$ to 1,3 -diphenyl- $\alpha, \beta-$ diketone $14(R=X=H)$. Since then, investigations have been 
extended to a large number of $\alpha, \beta$-epoxyketones by Zimmerman (22), Reusch (23), and Jeger (24). Recent studies have revealed that photolysis of $\alpha, \beta$-epoxyketones containing an alkyl group bonded to a carbonyl function yield predominantly $\beta$-diketones, whereas those having a phenyl group and a $\gamma$ hydrogen atom suitably orientated for hydrogen abstraction yield $\alpha$-hydroxyketones. In contrast to trans-dypnone oxide $15 \mathrm{a}$ and dimethylacrylacetophenone oxide 15b, which yield the respective $\alpha$-hydroxy ketones, 1,3-diphenyl-3-butene-2-ol-1one and 1-phenyl-3-methyl-3-butene-2-ol-1-one (22), irradiation of 3,4-epoxy-4-pheny 1-2-pentanone 16a and 3,4epoxy-4-methyl-2-pentanone $16 \mathrm{~b}$ afford the corresponding $\beta$ diketone derivatives $\underline{17 a}$ (22) and $\underline{17 b}$ (23). The former rearrangement, involving a 1,2 shift, has provided examples of

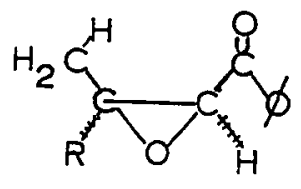

15 $a \cdot R=\varnothing$

b. $\mathrm{R}=\mathrm{CH}_{3}$<smiles>[R]C12CC(C)(C)CC(=O)C1(I)O2</smiles>

182. $R=\varnothing$

b $\mathrm{R}=\mathrm{CH}_{3}$

$\phi=\theta$<smiles>[R]C1(C)O[C@H]1C(C)=O</smiles>

16a. $R=\varnothing$

b. $\mathrm{R}=\mathrm{CH}_{3}$<smiles>[R]O[C@H]1C[C@]2(C)CC(=O)[C@H]1C2</smiles>

19a. $R=\varnothing$

b. $\mathrm{R}=\mathrm{CH}_{3}$ $\stackrel{\text { hN }}{\longrightarrow}$<smiles>[R]C(=O)C(C)C(C)=O</smiles>

17a. $R=\varnothing$

b. $\mathrm{R}=\mathrm{CH}_{3}$

$19 c$ 
relative migratory aptitudes different from those normally encountered for free radical processes. Reusch and coworkars (23) have also isolated 2-benzoyl-4,4-dimethylcyclopentanone 19a from $18 \mathrm{a}$ and 2-acetyl-4,4-dimethylcyclopentanone $19 \mathrm{~b}$ and 2,5,5-trimethylcyclohexan-1,3-dione 19c from isophorone oxide $18 \mathrm{~b}$.

The photoisomerization of 16 and 18 may be provisionally described by two main consecutive steps; first a cleavage of the C-O bond of the epoxide followed by a 1,2-shift of hydrogen or alkyl group to the $\alpha$-position. The first step of the photoreaction is analogous to the photochemically induced fission processes followed by the extrusion of electronegative substituents from the $\alpha$-position (or from a vinylogous position) of a carbonyl group (26-29). To account for the rearrangements, Zimmerman and co-workers (22,32) have interpreted the results as evidence supporting their description of the photochemical reaction as a consequence of $n \pi *$ excited state in ketones. Accordingly, of the alternative primary processes which can lead to either a heterolytic or homolytic dissociation of a C-O bond, they favour the latter process. Moreover, from the occurrence of a C-O fission, they have concluded that the carbonyl carbon of the excited state has an odd electron capability, suggestive of an electron rich carbon. The rearrangement has also been considered to be consistent with a free radical rearrangement, by analogy with the preferential extrusion of methyl over 
phenyl from a cumyl radical to yield acetophenone and a methyl radical (33). The mechanistic scheme proposed by Zimmerman is the following:

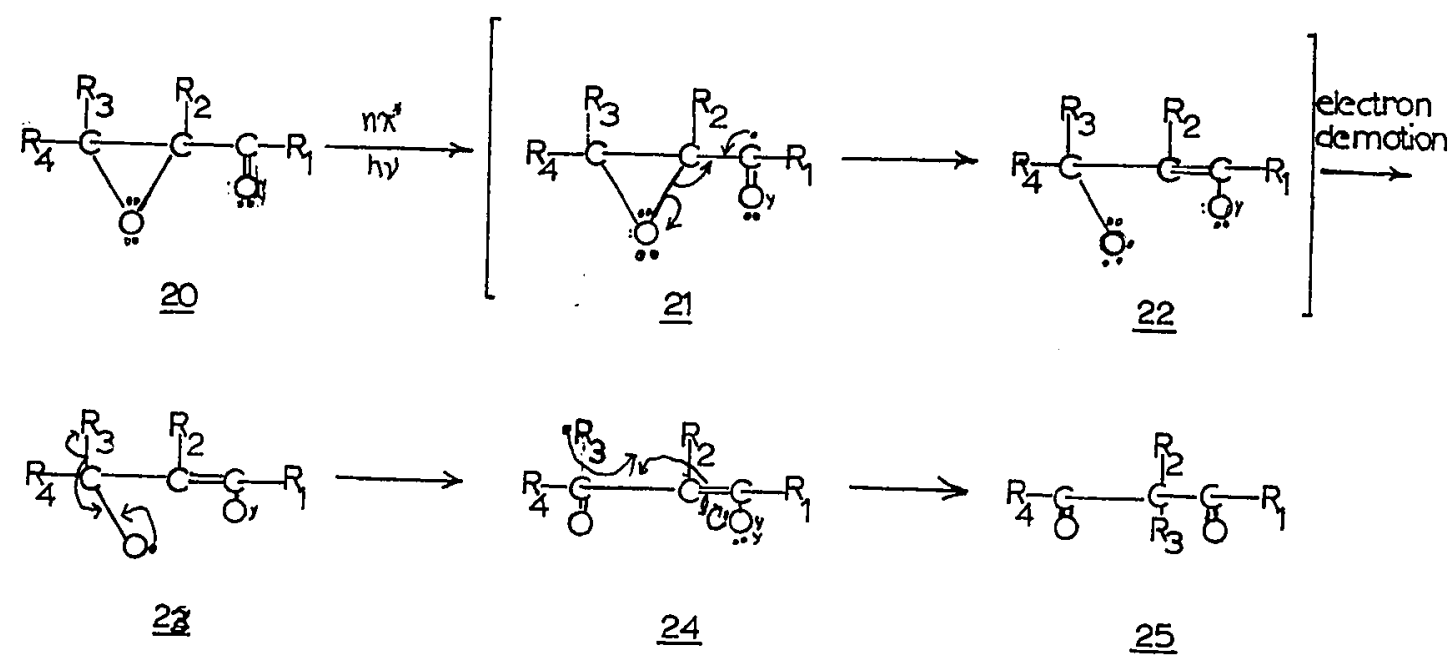

\section{Reaction 5}

Although the scheme above does account for the overall reaction in the aliphatic series, the attainment of stereot: specific reaction from analogous compounds of both saturated and unsaturated 3-oxo-4,5-epoxy-10ß -methyl steroids cannot be entirely accommodated by Reaction 5 without assuming a considerable amount of constraint in the possible transition states or intermediates. To demonstrate this point, Jeger $(36,37)$ has shown that photolysis of 4-methyl-4,5-oxido-17 $\beta$ acetoxy-androstan-3-one $\underline{26 \mathrm{a}}$ and $26 \mathrm{~b}$, rearrange. to $\beta$-diketones $\underline{27 \mathrm{a}}$ and $27 \mathrm{~b}$, differing only in their configuration at $\mathrm{C}_{4}$. 
When irradiated, $1 \alpha$-and $1 \beta-2$-oxido- $17 \beta$-acetoxy-5 $\beta$-androstan-3-one 30 are also stereospecifically converted to 33 and 34 , respectively.

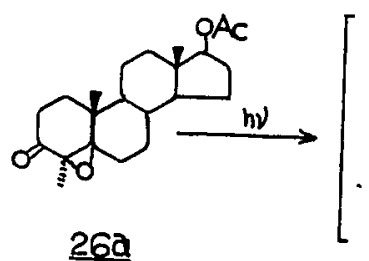

26a

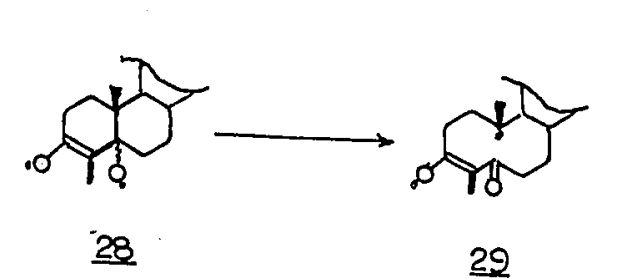

22

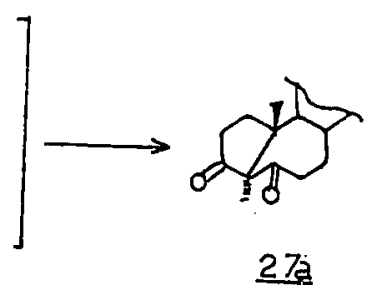

27

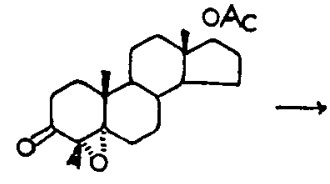

$26 b$

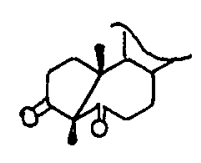

$27 b$ $\neq$

272

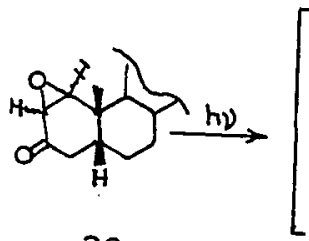

30

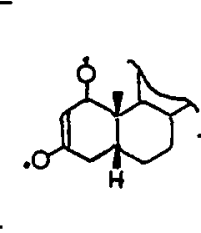

31

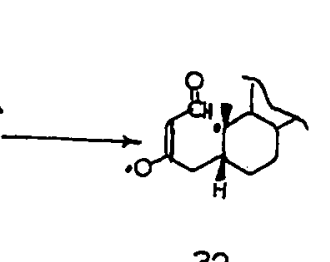

32

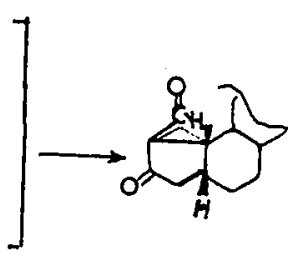

33<smiles>CC12CC3CC(CC(C3)C1CC(=O)O)C2</smiles>

34

\section{Reaction 6}

In the foregoing examples, 26a, 26b, and 30, if the rearrangement is to be adequately defined by Zimmerman's mechanistic scheme, one must assume that the configuration at $C_{10}$ is retained in $\underline{29}$ and 32 despite their radical 
character during the entire course of the rearrangement, or envisage the whole transformation as a concerted process without relaxation of the radical centers to a planar configuration.

The recently discovered light-effected isomerization of cyclopropanes and propenes in which an apparent migration of hydrogen, alkyl, aryl and acyl groups is observed, suggests that these reactions might be a common phenomenon with diradical intermediate initiated photochemically. When illuminated in solution, trans-12-diphenylcyclopropane 3 ' afforded predominantly cis-1,2-diphenylcyclopropane 32 and both cis- and trans-1,3-diphenylpropene 33 and 34 (39a). The genesis of these propenes from 31 and 32 , and $i$ ts reversion to the starting materials, involved an apparent 1,2-migration of a hydrogen atom, as observed in the photochemical isomerization of o,B-epoxy-ketones. Analogous reactions involving a 1,2hydrogen shift have also been observed for styryl epoxides (39d), and both 1,2 and 1,4 migrations have been detected with 1,2-indenoxides (39d) and 1,2-dimethyl-3-phenylcyclopropane (39e). Stilbene oxides do not undergo similar reactions (5I).

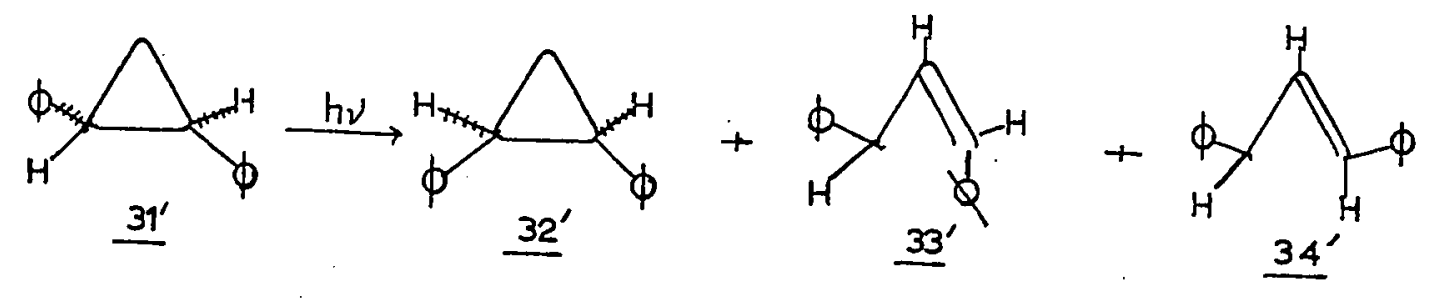


Further investigations in this area have been extended to include reactions of methyl (39c), phenyl (39c), and benzoyl (42) groups. In the rearrangement of the last group, viz., 41 to $42 a$, the migration of benzoyl rather than phenyl was shown to occur by isotopic labelling of the $\alpha$ carbon and one of phenyl groups in 41 . To account for both structural and geometricalisomerization of propenes and cyclopropanes, Griffin (39) has proposed, as the steps in the mechanism, the formation of diradical intermediates, followed by a rapid recombination of radical centers, or rearrangement by a facile intramolecular migration of a group. Evidence in support of this view has been provided by schuster and Krull $(40,41)$ who demonstrated that exposure to light of spiro-(2,5)-octa-4,7-diene-6-one-1,1,2,2-d 443 afforded, as one of the reaction products, a compound consistent with structure 45 in which the deuterium content was essentially retained.

In contrast to the ground state chemistry of free radicals, in which there is an apparent lack of authenticated hydrogen or alkyl migration $(33,34,35)$, these results obtained from irradiation of cyclopropanes and propenes are of current interest. Moreover, that these rearrangements are of a photochemical nature has been demonstrated by the complete absence of 1,3-diphenylpropenes $33^{\prime}$ and $34^{\prime}$ under conditions in which geometric isomerization of 1,2-diphenylcyclopropane is thermally induced (43). 

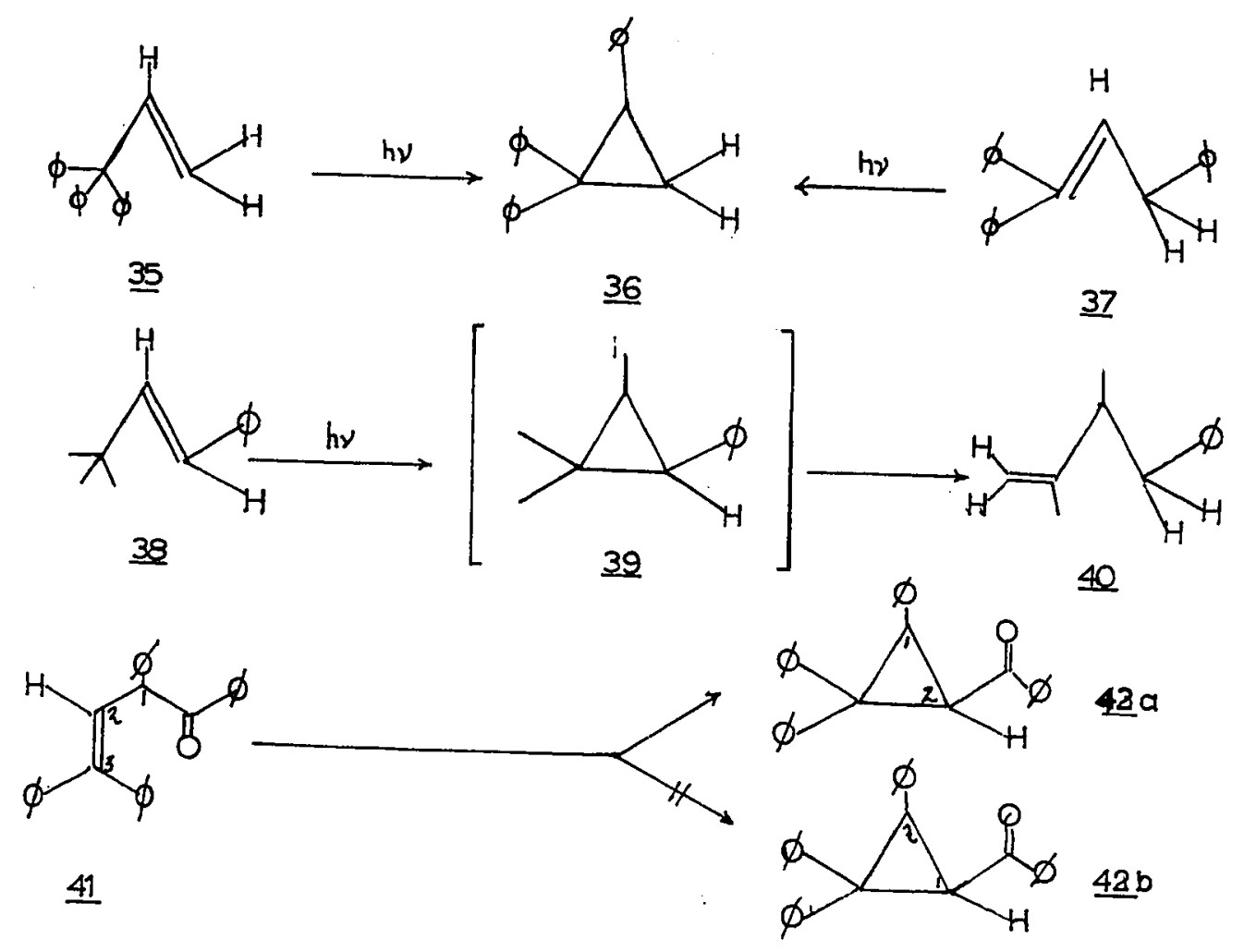

\section{Reaction 8}

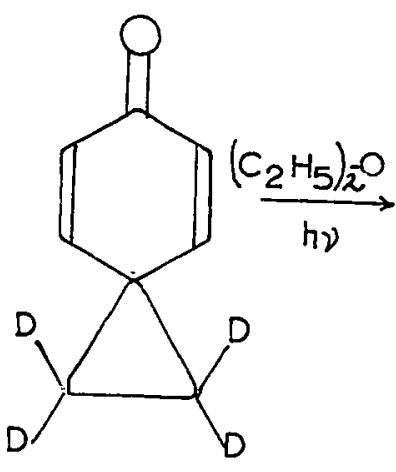

43

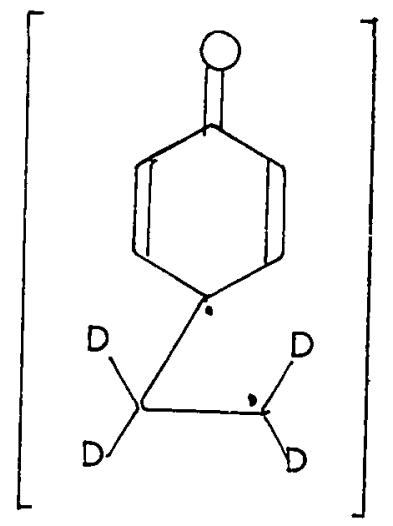

44

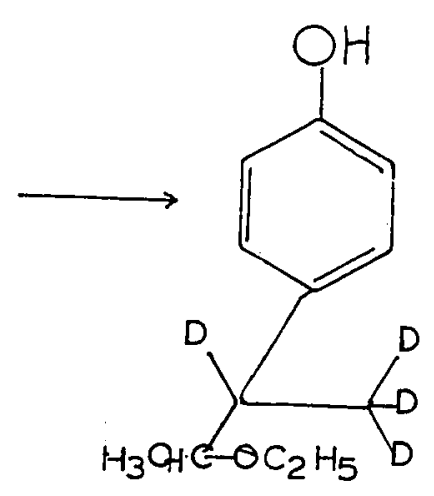

45 
The explanation for these photochemical isomerizations, in which the formation of diradicals is postulated, is not without precedent. Pitts and Norman (44) invoked such a mechanism to explain the conversion of acetylcyclopropane to 2-penten-4-one in the vapour phase. It remains to be demonstrated, however, that diradicals are really involved as intermediates in these reactions. Reports are available in the literature of unsuccessful attempts to trap the possible diradical intermediates of 1,2-dibenzoylcyclopropane and I-benzoyl-2-phenylcyclopropane under conditions of light induced geometrical isomerization $(49,50)$. In the vapour phase irradiation studies, oxygen has been found to be an ineffective bimolecular quencher of the mercury triplet sensitized reaction of 1 -butene to methylcyclopropane (47). It is well known that reactions in which free radicals or molecules, having sufficiently long life in their triplet states, are usually sensitive to the influence of oxygen.

Presently, no general assignment of the multiplicity of states responsible for the isomerization can be made. In the vapour phase, apparent 1,2 shifts of hydrogen have been shown to occur upon sensitization by mercury and benzene $(46,47,48 a)$. The workers who have made these observations regard the excited, rearranging species as being in the triplet state. However, in the condensed phase, sensitization of 1,2-diphenylcyclopropane by triplet donors has led 
exclusively to geometrical isomerization (45). In comparison to the vapour phase reaction, Griffin favours the assignment of a singlet state for the intermediate in the conversion of I,2-dimethyl-3-phenyl- cyclopropane 46 to 1-pheny 1-2-methylbut -I-ene 47 and 3-methyl-4-phenyl-but-l-ene 48 (39c,f), based on the fact that quenching by piperylene, which is an efficient triplet quencher, was without effect on the rearrangement.

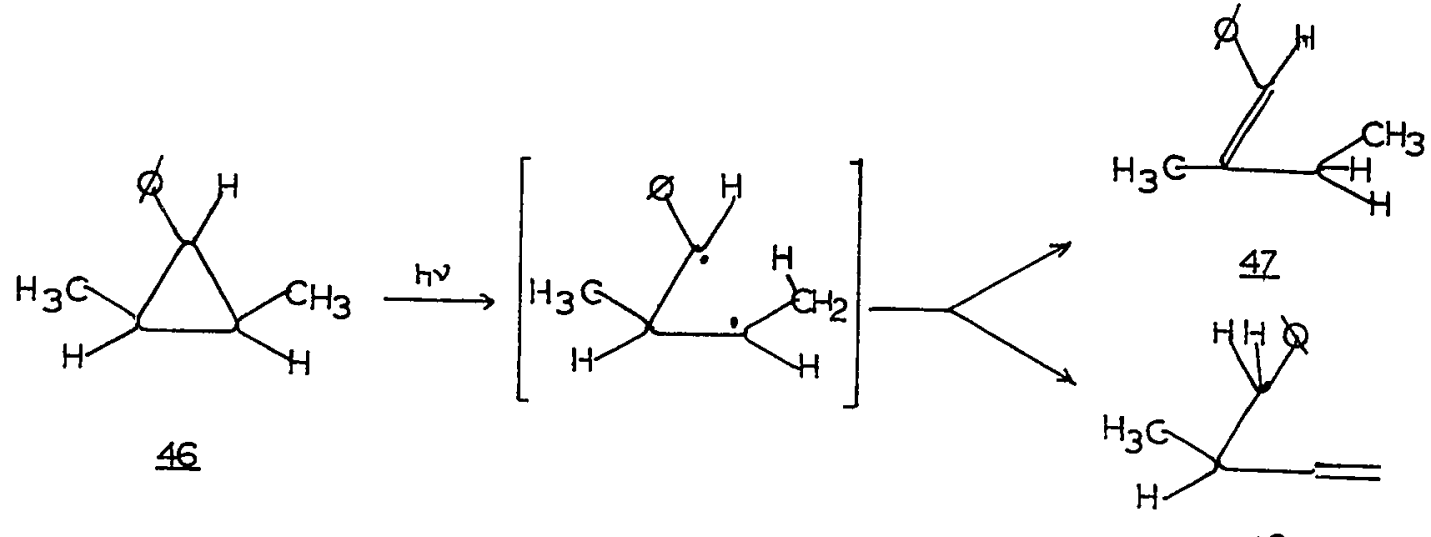

$\underline{48}$

\section{Reaction 10}

\section{c) Heteroatom Rearrangement}

In contrast to the previous class of photochemical transformations, numerous publications have appeared on photochemical reactions involving an apparent migration of a heteroatom. This category of reactions may be represented by Scheme III. Since it bears directly on the present investigation, it is reviewed extensively. 
Organic molecular system III (Scheme III) and its vinylogs have been found to be generally susceptible to photochemical rearrangement with an over-all migration of $C$ from $B$ to $A$.

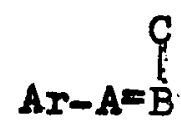

III

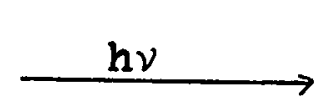

Scheme III
$\stackrel{C}{\perp-A-B}$

IV

Included in this classification are the transformations of azoxybenzene $\left(A=N, B=\mathbb{N}-C, C=0^{-}\right)$to hydroxyazobenzene (52-59), oxythiobenzoyl chloride $\left(\mathrm{A}=\mathrm{C}-\mathrm{Cl}, \mathrm{B}=\stackrel{+}{\mathrm{S}}, \mathrm{C}=0^{-}\right.$) to benzoyl chloride $(60-62)$, N-oxides $\left(A=C, B=\stackrel{+}{N}, C=0^{-}\right)$to amides (64-88), methyl isamate $\left(A=C, B=N^{+}, C=N^{*}\right)$ to a quinazoline derivative (63), and oximes ( $A=C, B=N=C, C=O H)$ to amides $(89,90)$. It is the last group which is of interest in the present research.

Since the first report of the sensitivity of $\alpha$-azoxynaphthalene to light in 1901 by Walker, the investigations of light-induced transformation of azoxy systems have been directed mainly towards determining the structures of the products. In 1925, Cunningham correctly assigned the 
structure to the photochemical product from $\alpha$-azoxynaphthalene as 2-hydroxyazonaphthalene (55-57), rather than as 4hydroxyazonaphthalene which had been previously assigned by Braundish and Furst in 1912 (53). In 1903, Knipscheer (54) established that azoxybenzene could be converted to 2-hydroxyazobenzene, and this conclusion was later confirmed by Cunningham (55-57), who subsequently studied a large number of azoxybenzenes. In 1954, Badger and Buttery $(58,59)$ reinvestigated the work of Cunningham, and, in addition to confirming the structures of the irradiation products from azonaphthalenes, they were able to establish that the oxygen atom initially attached to nitrogen apparently migrates to the ortho position of the non-adjacent aromatic ring. Compounds selected for this demonstration were, 4bromoezoxybenzene 49 and the $\beta$-isomer 51 and $\alpha, 2-p h e n y 1-$ azoxynaphthalene 53 and its $\beta$-isomer 54. Products from 49 and 51 were bromohydroxyazobenzenes 50 and 22 , respectively.

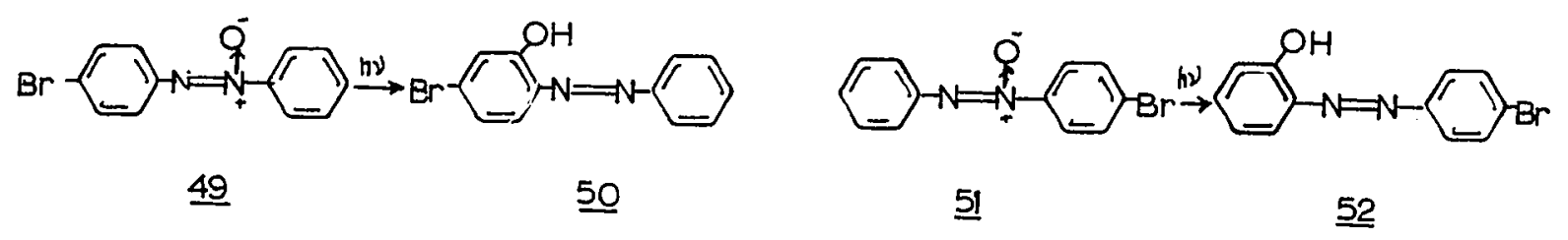

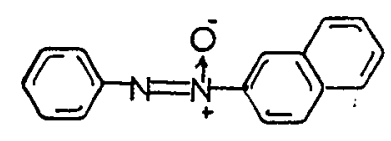

$\underline{53}$

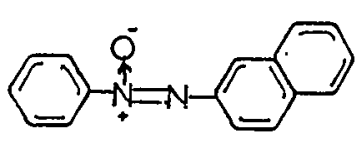

$\underline{54}$

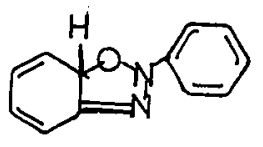

$\underline{55}$ 
The exclusive formation of ortho hydroxyazo derivatives was interpreted to be indicative of an intramolecular rearrangement of oxygen from the nitrogen atom to the ortho position of the non-adjacent aromatic nucleus due to favourable spatial orientation. A possible transient species 55, has been suggested (ref. 1,p. 393, 58b).

Less extensively studied reactions of system III are the photochemical rearrangement of s-oxides (such as oxythiobenzoyl chlorides 56) reported by King and Durst (60, 6I), and that of 2-methoxynaphthalene-1-thiocarbaldehyde-Soxide $56 \mathrm{~b}$ reported by Strating and co-workers (62). When irradiated, $56 \mathrm{a}$ is converted to $\underline{5 \mathrm{a} a}$, and $\underline{56 \mathrm{~b}}$ to $\underline{58 \mathrm{~b}}$. If these photochemical conversions are entirely analogous to the rearrangement of nitrones, and are intramolecular, the reaction may be represented in the following manner:

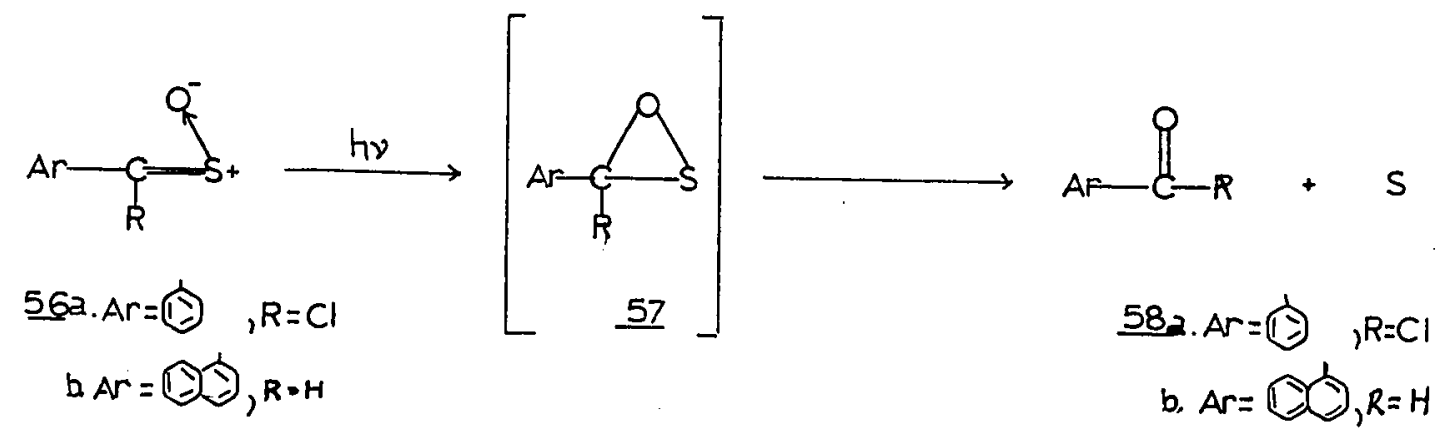

Reaction 12

Recently, deMayo and Ryan (63) have discovered an 
analogous reaction. When methyl isamate 59 is irradiated, the product is a quinazoline derivative 61 . The intermediacy of a diaziridine 60 has been suggested.

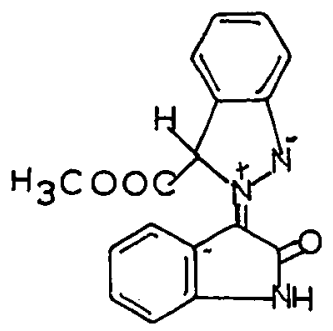

$\underline{59}$

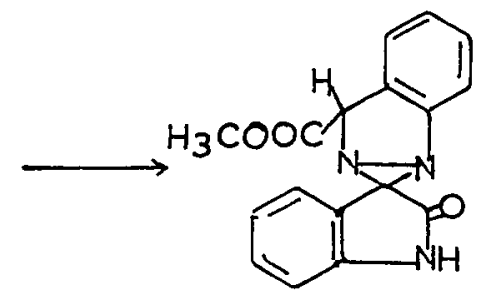

$\underline{60}$<smiles>CC(=O)C1C=NC2=NC(=O)c3ccccc3NC2=N1</smiles>

61

\section{Reaction 13}

The most extensive investigation of system III (Scheme III) has been confined to nitrones and their vinylogs. In 1910, Alessandri (64) first reported the isolation of benzanilide and hydrolysis products upon irradiation of $N$, a-diphenylnitrone. Since then, numerous examples have appeared in the literature (64-81). Irradiation of a nitrone 62 , gives an oxazirane 63 by internal addition, which subsequently rearranges to amides $\underline{64 \mathrm{a}}$ and $\underline{64 \mathrm{~b}} \mathrm{de-}$ pending upon the stability of the oxazirane intermediate $63(66-72)$. Oxazirane was considered by early workers $(67,70,71)$ as a possible intermediate and this was later confirmed in 1958 by Splitter and Calvin (67) who were able to make a direct comparison of the irradiation products 
from the nitrones with oxaziranes prepared by peracid oxidation of imines. The chemical preparation of oxaziranes<smiles></smiles>

Reaction 14

has become known largely through the efforts of Krohnke (71), Horner (79) and Emmons (77-78) in 1957. In 1959, Todd and co-workers (74a) also prepared a stable oxazirane 66 photochemically from 5,5-dimethyl-pyrrolidine-N-oxide $\underline{65}$ and chemically from 5,5-dimethyl-pyrrolidine 67 .<smiles>CC1(C)CCC=[N+]1[O-]</smiles>

65

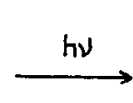<smiles>Cc1cc(Br)cc(C)c1/C=[N+](/C)[O-]</smiles>

$\underline{68}$

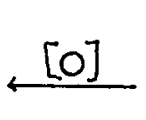

65<smiles>CC1(C)CCC=N1</smiles>

67<smiles>[CH-]C</smiles><smiles>Cc1cc(Br)cc(C)c1C1ON1C</smiles>

69 
Recently, Jerslev $(75,76)$ using $x$-ray techniques has determined the structure of 2-methyl-3-(4-bromo-2, 6-dimethylphenyl) oxazirane 69, obtained by irradiation of the corresponding nitrone 68 .

Vinylogous extension of the photochemical rearrangement of nitrones has included the reactions of isoquinoxaline (82), purine-N-oxide $(83,84)$, isoquinoline-N-oxides $(85 \mathrm{a}, \mathrm{c})$, quinoline-N-oxides $(85,86)$, and pyridine-N-oxides (87). The earliest investigation in this series was reported in 1953 by Landquist and Stacey (82a) who isolated 2-quinoxalinol 71 from irradiation of isoquinoxaline-l-oxide 70 . Kaneko and co-workers ( $82 \mathrm{~b}$ ) recently concluded that an oxazirane is involved in the reaction and in one instance. it has been isolated.<smiles>[O-][n+]1ccnc2ccccc21</smiles>

70

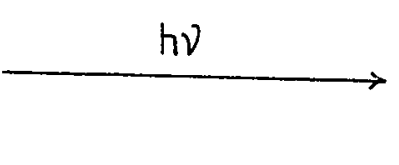

Reaction 16

Within the last five years, there has been a renewed interest in this area of research, as exemplified by the application of the reaction to other systems related to that of quinoxaline-l-oxide. Brown and co-workers (83) isolated an oxygen-transfer product, 2-hydroxyadenine $73 \mathrm{a}$ when 
adenine-l-oxide $72 a$ was irradiated. Similarly, isoguanosin 73b was isolated from adenosine-l-oxide 72b (84).

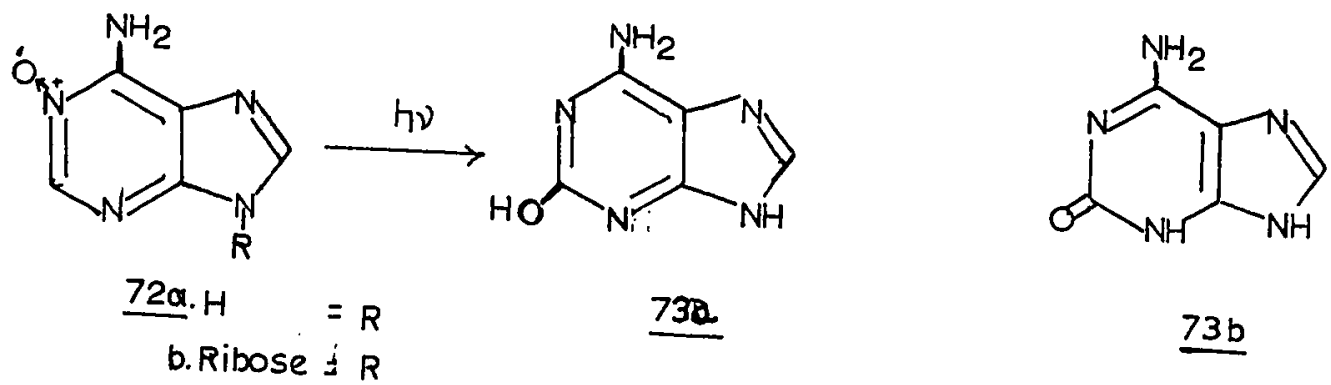

Reaction 17

On the other hand, quinoline-N-oxides and isoquinoline2-oxides have been actively investigated $(85,86)$. Except for 2-substituted quinoline-N-oxides, the products obtained from quinoline-N-oxides are the expected 2-carbostyril derivatives, arising from the thermal rearrangement of the initially formed oxaziranes (85a). Under suitable conditions, the intervention of oxaziranes has been demonstrated (85). A few examples are given in Reaction 18.<smiles>[R]c1cc(C=NC)[n+]([O-])c2ccccc12</smiles><smiles>[R9]OC</smiles>
$\mathrm{bR}=\mathrm{OCH}_{3}$

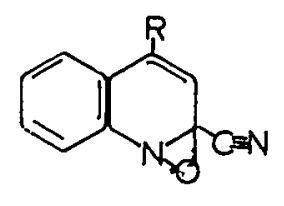<smiles>[R]c1cc2ccccc2c(C#N)[n+]1[O-]</smiles><smiles></smiles><smiles>COC(C)C(C)Cl</smiles>
$\underline{77} \mathrm{R}=\mathrm{H}, \mathrm{CH}_{3}$ 
In addition to the fact that the solvent influences the rate of reaction and the nature of products, startling effects are caused by substituents situated at the carbon adjacent to the nitrogen. 3-Cyano-4-methoxyquinoline-N-oxide 74b, when irradiated, rearranges to 2-cyano-3-hydroxy-4-methylquitioline 78b A pathway involving two successive 1,2 shifts has been suggested by Ishikawa et al (85). This is due to the thermal rearrangement of oxazirane $750\left(\mathrm{R}=\mathrm{CH}_{3}\right)$. Oxazirane $75 a\left(\mathrm{~B}=\mathrm{CH}_{3}\right)$ actually rearranges thermally to $28 \mathrm{a}\left(\mathrm{R}=\mathrm{CH}_{3}\right)$.

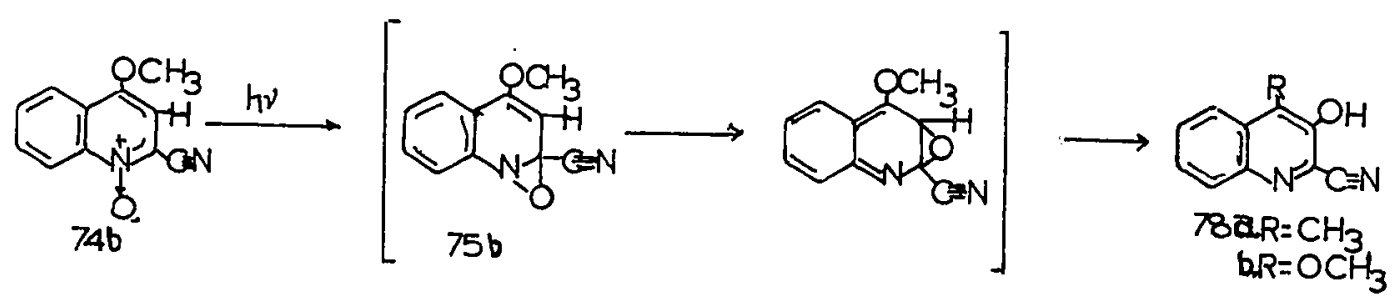

Reaction 19

Furthermore, the substitution of the quinoline-N-oxide with a methyl group on carbon-2 alters the course of reaction to the extent that at least five different products are obtained by irradiating 79 in methanol ( $85 \mathrm{a}, \mathrm{e}, 86 \mathrm{c}, \mathrm{d}$, $87 b)$. The reaction products are given below in Reaction 20. The formation of these products can also be rationalized in terms of the intermediacy of an oxazirane.

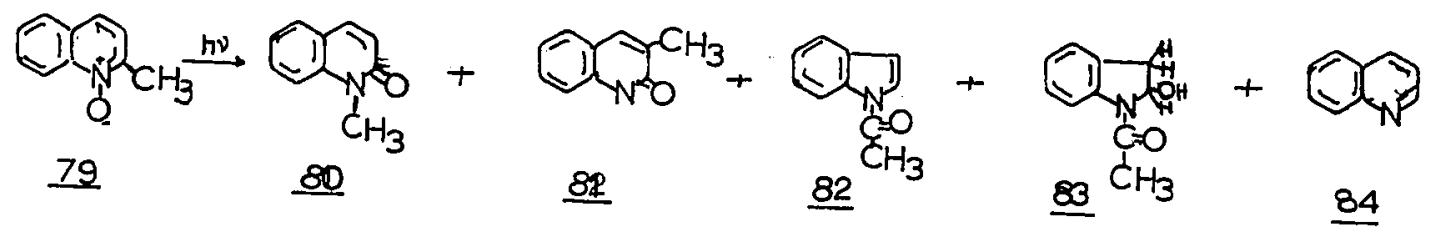


In the absence of the second ring of the quinoline-Noxide, the reaction appears to follow another pathway. In addition to the expected $\alpha$-pyridone 88 a Streith and Sigwalt (87a) have isolated 2-formyl pyrroles 89 which they suggest arise from ar oxazepine 87, and $i$ ts valence tautomers $\underline{8 \mathrm{a}}$ and $\underline{88 \mathrm{~b}}$, as depicted in Reaction 21 .

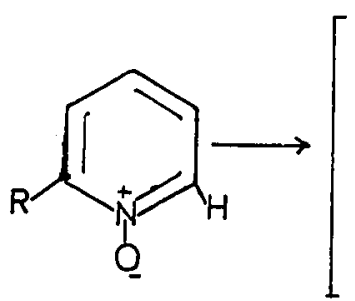

85. $\mathrm{R}=\mathrm{H}_{1} \mathrm{CH}_{3}$<smiles>[R]C1=CC=CC2ON12</smiles>

86

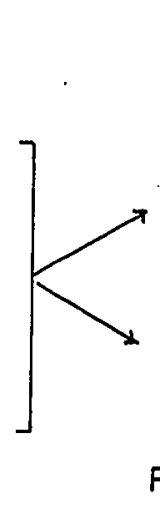

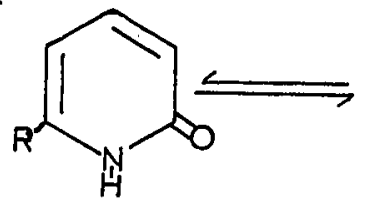

889

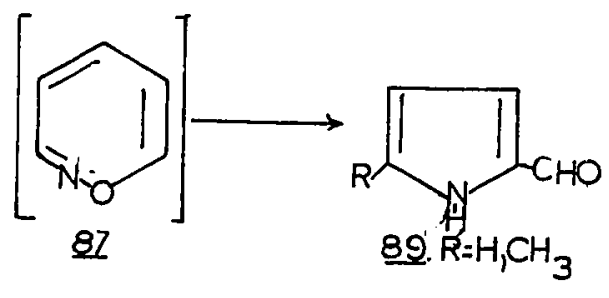<smiles>c1ccncc1</smiles>

836

\section{Reaction 21}

Recently Kaneko (85d) and Buchardt (86e) have suggested that an oxazepine could intervene in the over-all conversion of some of the quinoline-N-oxides. The latter author* then actually claims that oxazepines are formed as stable products.

Although there have been numerous reports of photochemical rearrangements of system II (Scheme III), littie attention has been directed towards defining the mechanism of the reactions. When studies of benzaldoximes aimed at elucidating the mechanism of the photochemical Beckmann rearrangement. were initiated in our laboratories, no

*After the thesis was written, C. Kaneko, S. Yamada, I. Yokoe and M. Ishikawa (Tetrahedran Ietters, 1833 (1967) 
detailed work on the mechanism of System III had been reported except for that of Hata and Tanaka. (88). They had conducted some research on the vapour phase photolysis of pyridine-N-oxides. Their work has had considerable relevance to the light-effected reaction of quinoline-N-oxides and pyridine-N-oxides in the condensed phase subsequently reported by Buchardt (86), Ishikawa and Kaneko (85), and Streith (87).

Hata and Tanaka ( $88 \mathrm{c}$ ) reported that the photochemistry of pyridine-N-oxide and that of 3-picoline-N-oxide are very similar. The products of the photolysis are the corresponding pyridines as a result of deoxygenation, and gases (hydrogen, oxygen, and carbon dioxide) formed by decomposition. The reaction is not wavelength dependent. From their results, they interpreted the chemical processes of these $\mathbb{N}$-oxides in terms of $n \pi^{*}$ or $\pi \pi^{*}$ electronic transition states, and assumed that the excited state in its triplets dissociated into pyridine and an oxygen atom. On the other hand, the photolysis product of 2-picoline-N-oxide is wavelength dependent, in that irradiation only in the $\pi \pi^{*}$ region of the electronic transition band gives similar reactions. Irradiation in the $n \pi^{*}$ region, instead of affording 2-picoline, yields 2-pyridylmethanol, retaining its oxygen atom. The formation of 2-pyridylmethanol has been suggested to be a consequence of the population of the triplet state followed by an intramolecular rearrangement. The $n \pi *$ transition of the electronic state from which re- 
arrangement ocours in the high wavelength region of the ultraviolet spectrum asid has been attributed to the trans1610n of a non-bonded electron on the oxygen atom to the higher $\pi^{*}$ molecular orbital. The $\pi \pi^{*}$ band 18 assoclated with the transition of the $\pi$ electron to higher $\pi^{*}$ orbitals.

The first detailed investigation of system III (Scheme III) In the liquid phase was reported by Tanaka and ooporkers $(68,69)$ who studied the chemical rearrangement of $\alpha, N$-diphenglnitrone $20 a$ and $\alpha$-cyano- $\alpha$-phenyl-N-phenylnitrone $20 \mathrm{~b}$. In both instances, the primary product of the photochemical reaction is the corresponding oxazirane 21. The oxazirane 21 a subsequently rearranges thermally to the benzanilide 23. The primary reaction has been found to be Insensitive to oxygen and temperature but noticeably sensitive to protonic solvents. On the basis of these results, the rearrangement process has been interpreted in terms of a rap1d intramolecular movement of the oxygen atom to the $\alpha$ carbon upon excitation to the singlet state. The significant change in the dipoles of the N-O bonds and the appreciable increase in the free valency of the $\alpha$-carbon atom upon excitation to the singlet state are regarded as the driving forces of the rearrangement. Bxperimental evidence for the assignment of the singlet multiplicity of the excited state has been supported by the fact that, whereas direct irradlation of $20 \mathrm{~b}$ leads to the formation of an oxazirane $21 \mathrm{~b}$, sensitized 1rradiation with efficient triplets donors initiates 
only geometrical isomerization.

As will become clear, the rearrangement of benzaldoximes possesses some of the characteristics of the nitrone transformation but differences are nevertheless apparent.

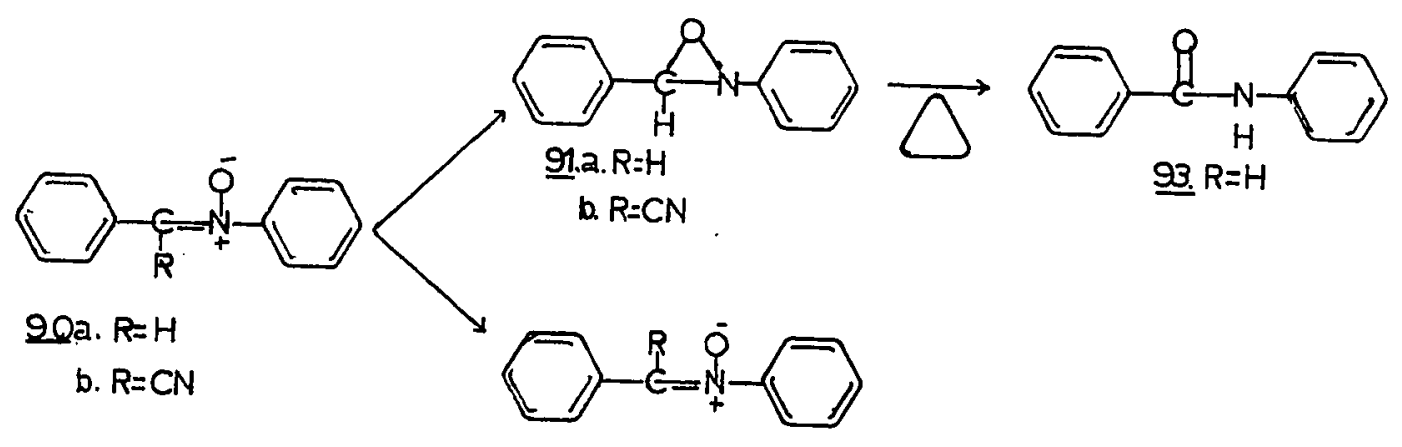

92. $\mathrm{RCN}$

\section{Reaction 22}

Because this thesis is concerned mainly with the lightinduced Beckmann rearrangement, a brief discussion of the mechanism of the reaction in the electronic ground state is warranted. Since its discovery in 1886 by H. Beckmann (9I), numerous publications on the subject have appeared, indicating the sustained interest of many workers in elucidating the mechanism(94). Recent reviews on its scope and limitation, as well as on the mechanism, have been presented by Heldt (92) and Smith (93). The Beckmanr. Rearrangement is an acidcatalyzed transformation of oximes to their corresponding amides in a highly stereospecific way. From the accumulated 
knowledge about the reaction, a formal reaction scheme can be presented in the following manner, taking as a typical example, a protonic acidbatalyzed reaction:

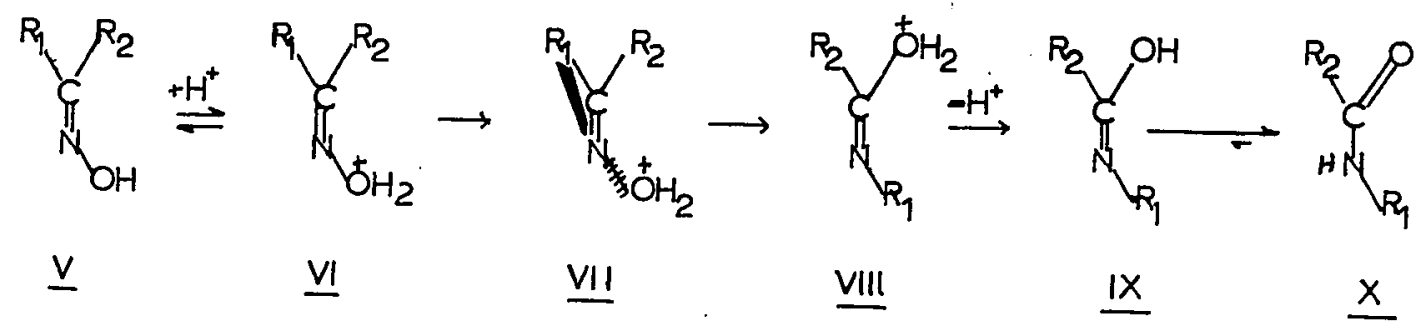

Scheme IV

In this scheme, $R_{1}$ and $K_{2}$ can represent hydrogen, alkyl or aryl groups. Since the esters of the oximes also undergo an extremely facile rearrangement, $\mathrm{OH}_{2}^{+}$can be replaced by the conjugate base of a strong acid (OA). The cleavage of $O A$, VII $\longrightarrow$ VIII, can be envisaged as an internal displacement of a conjugate base. This process might be termed as "ionization by displacement". There are sufficient data available to indicate that VII is a highly energetic species with a substantial charge separation (91-93). It may be a true intermediate or a transition state. The mechanism of the reaction consists essentially of the formation of an electron deficient nitrogen by the partial ionization of the $\mathrm{O} N$ bond of the oxime, with a concerted intramolecular migration of a group anti or trans to the departing moiety ( $\mathrm{OH}_{2}^{+}$or $\mathrm{OA}$ ), by 
heterolytic cleavage (93-96). Pertinent to the present study of the photochemical rearrangement is the molecularity of the normal Beckmann rearrangement in the electronic ground state. There is evidence that the over-all conversion involves an intermolecular migration of the oxygen atom. This has been provided by Brodskii (97) who demonstrated that $18_{0}$ is incorporated into benzanilide when benzophenone oxime rearranges under the Beckmann reaction conditions in the presence of ${ }^{18} \mathrm{O}$ enriched water. Furthermore, the results of cross-over experiments, conducted by Smith (ref. 92, p.45) with benzophenone oxime picryl ether 24 and $p$, p-dimethylbenzophenone-3-methylpicryl ether 25, give supporting evidence for the intermolecularity of the over-all reaction. of further interest in connection with the present work is the dependence of the course of the reaction on electronic effects. According to the mechanism outlined in scheme IV, the rearrangement should be consistent with diagnostic tests for the intervention of a charge separated species. Invariably, this has been confirmed experimentally. The rate of reaction of oxime esters increases with increasing stability of the conjugate base $(O A)$. A negative rho value has been obtained from a Hammettplot for compounds containing various substituents on the migrating group. Furthermore, the rate reaction has been correlated with the ionizing power of the solvents, $\nabla 12$. the reaction is facilitated by polar solvents $(92,93)$. 


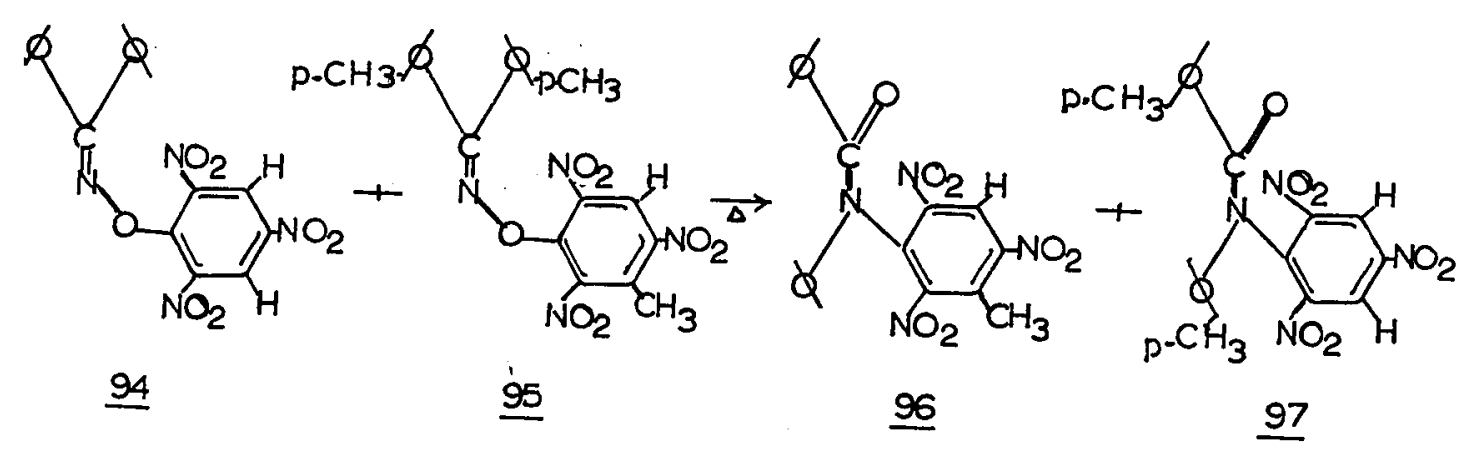

Reaction 23 


\section{INTRODUCTION}

In 1963, Amin and da:Mayo (89) found that benzaldoximes 98 are transformed photochemically into the corresponding benzamides 29, with an apparent 1,2 migration of the oxygen atom on nitrogen to the adjacent carbonyl carbon, as observed in the normal acid-catalyzed rearrangement of benzaldoxime $(92,93)$. At thet time, only a preliminary account of this reaction had been given without elaboration of the mechanism.

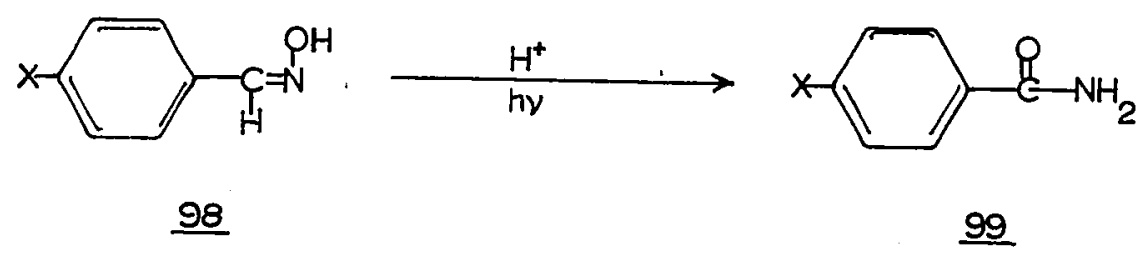

Reaction 24

The only quantitative result available at. that time, was the yield of the reaction obtained by irradiation of the oximes with unfiltered light in a quartz immersion well. Therefore, the mechanism of the reaction had to be inter. preted with reservation. Furthermore, it had also been reported that only certain substituted benzaldoximes rearrange e.g. $98\left(\mathrm{X}=\mathrm{H}, \mathrm{CH}_{3}, \mathrm{CH}_{3} \mathrm{O}, \mathrm{CI}\right)$, and that those con- 
taining electronegative groups, such as $\mathrm{F}, \mathrm{C} \equiv \mathrm{N}$, and $\mathrm{NO}_{2}$ ' failed to yield amides. In addition, attempts to isolate amides from the corresponding arylketoximes in our laboratories had been unsuccessful. Also, with regard to substituent effects, a decision could not be reached as to whether the electronic influence modified the nature of the excited states or whether it affected the nature of the intermediate. Although an oxazirane intermediate was considered probable, no experimental evidence was available. Moreover, the multiplicity of the states responsible for the transformation required identification and the molecularity of the over-all reaction remained to be established.

The work reported here includes the investigation of the effects of solvents, substituents, oxygen and acids upon the quantum efficiency of the rearrangement. Dependence of the quantum yield of the rearrangement upon the wavelength of the exciting source and upon the sensitization by donor molecules, capable of transfering energy from their triplet state, was also studied. In addition, a cross-over experiment was conducted to establish the molecularity of the over-all reaction. As a further entry into this area of research, the problem was extended to include acetophenone oxime since the results from the investigation of the benzaldoximes indicated that similar transformations should be applicable to aromatic ketoximes. Spectroscopic evidence for the transfer of triplet energy from sensitizers to benzaldoxime is also presented. 
Some aspects of the geometrical syn-anti or cis-trans isomerization were investigated since this photochemical process proved to be the more efficient photochemical process than the rearrangement to the corresponding benzamide. The synthetic utility (119-112, 150, 151) of the irradiative conversion of oximes into their geometrical isomers has been recognized since 1903 when Ciamician and Silber (119) first observed the isomerization for aldoximes. Although half a century has elapsed, absolutely no reports of investigations directed towards the elucidation of the mechanism have appeared. In this research, stationary states by both direct and sensitized irradiation, as well as the quantum efficiency of geometrical isomerization in both directions were determined.

Based upon the experimental results, a plausible description of the photochemical Beckmann rearrangement, in accordance with the present photochemical theories, is given. 
RESULTS AND DISCUSSION

A. Molecularity of the Photochemical Beckmann Rearrangement

A cross-over experiment was designed to establish the overall molecularity of the photochemical conversion of benzaldoxime to benzamide. Irradiation with unfiltered light from a mercury lamp of an equimolar concentration of isotopically labelled benzaldoxime- ${ }^{18}$ O ( 1.54 atom per cent excess) and p-tolualdoxime yielded the two corresponding benzamides. The ${ }^{18} 0$ contents of benzamide and p-toluamide were 1.53 and 0.034 atom per cent excess, respectively. The synthesis of benzaldoxime- ${ }^{18} 0$ and the separation of the two amides are given in the experimental section.

The retention of ${ }^{18} 0$ content in benzamide, to the extent of $99 \%$ of the amount present in the starting material when both benzaldoxime- ${ }^{18} 0$ and $p$-tolualdoxime were irradiated together in acetic acid, is a clear indication that the overall photochemical Beckmann Rearrangement of benzaldoxime is intramolecular. Therefore, a description of the mechanistic pathways of the reaction must be devoid of any steps involving complete fragmentation of oxygen from any part of the benzaldoxime nucleus. Had there been any dismuption of the oxygen atom at any stage and of the dissociated groups being sufficiently long-lived then, one might have expected to find cross-over products, in which an appreciable amount of the 
180 content present in benzaldoxime- ${ }^{18} 0$ would have been

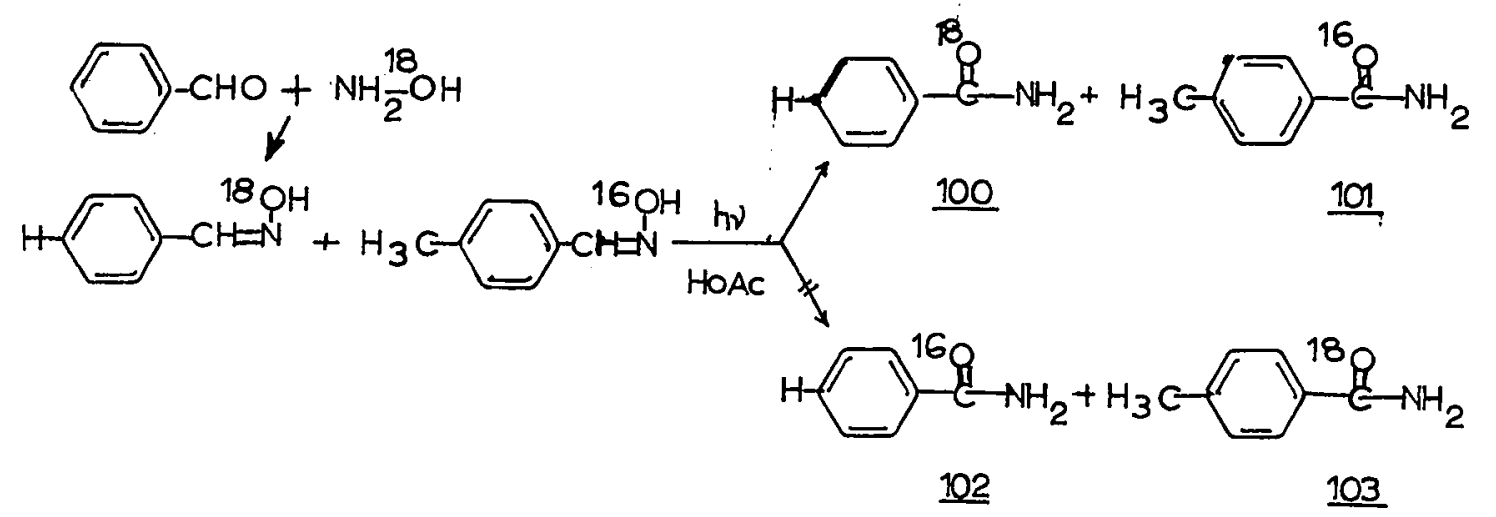

Reaction 25

incorporated into p-toluamide 103 with a corresponding dilution of the ${ }^{18} 0$ content in benzamide 102 .

In contrast to the light induced rearrangement, the normal acidcatalyzed reaction has been demonstrated to be intermolecular. This difference in the modes of conversion of benzaldoximes may be an indication that the photochemical pathway excludes any consequence of a prior internal conversion of the excited electronic energy to the higher vibrational levels of the ground state. Had the degradation of the initially absorbed radiant energy by the oxime been such that the same activated state of the normal acid catalyzed reaction was attained, one might have expected to observe similar results from both the photochemical and the normal acid-catalyzed reaction in the ground state. It is, 
therefore, probable that the photochemically induced conversion of the oxime is free of an imine intermediate, and that the transfer of the oxygen atom occurs directly in its electronically excited state in the analogous way postulated by Hata $(88 b)$ and Tanaka $(68,69)$ for the rearrangement of N-oxides.

It had been reported earlier (83) that, whereas benzaldoxime rearranges to benzamide upon irradiation, the corresponding acetate is unreactive. Chapman (98) states that exposure of benzophenone oxime picryl ether in benzene solution to the light of a mercury vapour lamp caused some decomposition but no rearrangement. This finding, in part, lends support to the view that an initially excited molecule of benzaldoxime acetate is not internally converted to a "hot" molecule in the ground state to initiate the normal Beckmann rearrangement. It is well documented that esters of oximes undergo a more facile transformation than the oximes themselves, and it has been suggested that one of the driving forces of the normal rearrangement might be the formation of an ester $(92,93)$.

\section{B. Intervention of an Intermediate}

It has been speculated previously by Amin and de:Mayo (89) that the conversion of benzaldoximes to benzamides could possibly involve the intermediacy of an oxazirane as 
has been observed in the light induced conversion of nitrones to amides. In fact, in several instances, such intervention of an oxazirane from a nitrone has been demonstrated by Splitter and Calvin $(67 \mathrm{~b}, \mathrm{c})$, Tanaka (68), and Ishikawa (85). The results of the cross-over experiment clearly established the intramolecularity of the light induced conversion of benzaldoxime to benzamide and also suggested that an oxazirane was a possible precursor of benzamide. Evidence is now presented for the intermediacy of an oxazirane.

The general set-up of the apparatus for low temperature irradiation in a fluid solution is schematically represented in Fig. 1. The technique employed was essentially that described by Hirschberg and Fisher (100). $\alpha$-p-Anisaldoxime was irradiated in $n$-hexane and the reaction was followed spectrophotometrically. The spectra, shown in figures 2,3 and 4 for the oxime, were recorded under two different sets of experimental conditions; in one, the photolyte was unmixed and in the other, the solution was mixed by bubbling a slow stream of nitrogen through it. Exciting light at $290 \mathrm{mp}$ was transmitted through parallel quartz windows of $1.0 \mathrm{~cm}$ in diameter.

Those spectra taken in cyclohexane and pertinent to the irradiation at low temperature are also recorded. Fig. 5 shows the ultraviolet spectra of $\alpha$-and $\beta$-p-anisaldoxime, 
p-anisamide and $\mathbb{N}$-p-methoxyphenylformanilide in cyclohexane and 2-ethyl-3-p-methoxyphenyloxazirane in ethanol. The last spectrum of the oxazirane is reproduced from the paper published by Splitter and Calvin (67c).

Thin layer chromotography (TIC) showed that the residue from irradiated solutions of p-anisaldoxime $\left(<-50^{\circ} \mathrm{C}\right)$, which had been allowed to stand at room temperature, contained both p-anisamide and p-methoxyformanilde. It was also observed that prolonged irradiation of the oxime usually results in diminished intensity of the absorption at $232 \mathrm{~m} \mu$ in the ultraviolet spectrum.

In view of the fact that oxaziranes, which do not possess any alkyl or aryl substituents on the nitrogen atom, are extremely unstable thermally and susceptible to acidic cleavage (II0-III), the logical choice of experimental conditions was to irradiate a benzaldoxime in a neutral solvent at a very low temperature. Under these conditions the extent of the reaction could be followed spectrophotometrically or the trapping of intermediate with suitable reagents could be attempted. Although attempts by Emmons (III) to prepare 3-phenyloxazirane by peracid oxidation of the corresponding imine had been unsuccessful, recently, Schmitz (110a) has reported that derivatives of it can be prepared by treatment of 3-phenyloxazirane with isocyanates or acyl halides. 
The compound selected for irradiation at low temperature was $\alpha$-p-anisaldoxime. This oxime was especially suitable for study since the ultraviolet spectrum of 2-ethyl-3-pmethoxyphenyloxazirane could serve as a guide to the type of spectrum which might be expected in the event that the corresponding 3-p-methoxyphenyloxazirane was formed (see curve 5, Fig. 5). The highest intensity of absorption of the model compound, 2-ethyl-3-p-methoxyphenyloxazirane, appears at $231-213 \mathrm{~m} \mu$ with a molar extinction coefficient of 13,800, suitably positioned in the region of the minima of both the starting materials ( $\alpha$ - and $\beta$-p-anisaldoximes) and the products ( $p$-anisamide and p-methoxyformanilide).

$$
\begin{aligned}
& \text { The results of irradiation of an unmixed solution of } \\
& \alpha \text {-p-anisaldoxime (see curve } 3 \text {, Fig. 2) at }-87 \text { to }-88^{\circ} \mathrm{C} \text { is }
\end{aligned}
$$
highly suggestive of the intermediacy of the corresponding oxazirane. A well-defined maximum appears at 231-232m the same position as that observed in the ultraviolet spectrum of the model compound. Moreover, this region of the spectrum has a minimum at $245 \mathrm{~m} \mu$. The appearance of the maximum at $232 \mathrm{~m} \mu$ is, therefore, a consequence of the photoconversion of p-anisaldoxime to another compound that is neither p-anisamide nor p-methoxyformanilide. The maxima in the ultraviolet spectra of both amides appear at 250 to $252 \mathrm{~m} \mu$ $(\epsilon=11,500-12,400)$. The positions of the maxima are essentially unaffected upon cooling. 
An estimate of the extent of conversion of $p-\alpha-$ anisaldoxime to oxazirane is possible from the spectroscopic data using equation 1 or 2 (107) is the following assumptions are made: a) the Beer-Iambert law is obeyed at the wavelengths and concentrations employed, b) the molar extinction coefficients are temperature independent, and c) the reaction mixture contains only the starting material, $\alpha_{-}$and B-p-anisaldoximes and the corresponding oxazirane. Both equations $I$ and 2 apply to a situation which is equivalent to that in which an oxazirane and only one of the isomeric oximes need to be considered since $\epsilon_{Q}=\epsilon_{\alpha}=\epsilon_{\beta}$ at an isosbestic point.

Eq. 1) $\quad\left(\frac{O D_{q}}{O D_{0}} \lambda_{i}=\quad Q+(I-Q) \frac{\epsilon_{Z}}{\left(\frac{\epsilon_{Q}}{\epsilon_{i}} \lambda_{i}\right.} \quad\right.$ or

Eq. 2) $Q=\left[\frac{O D}{O D} \lambda_{i}-\frac{Z}{Q} \lambda i\right]\left[\frac{Q}{Q-Z}\right] \lambda_{i}$ where

$\left(O D_{t}\right)_{i}=$ final optical density of the solution at wavelength $\lambda_{i}$

$\left(O D_{0}\right)_{\lambda}=$ initial optical density of the solution at wavelength $\lambda_{i}$

Q = mole fraction of anisaldoxime at wavelength $\lambda_{i}$

$(I-Q)=$ mole fraction of oxazirane 
$\left(\epsilon_{Q}\right)=$ molar extinction coefficient of p-anisaldoxime $\lambda_{i}$ at wavelength $\lambda_{i}$ at a isosbestic point $\left(\epsilon_{\alpha}\right.$ $\alpha$-anisaldoxime $\left.=\epsilon_{\text {Banisaldoxime }}\right) \lambda_{i}$

$\left(\epsilon_{Z}\right) \lambda_{i}=$ molar extinction coefficient of the oxazirane

Referring to curve 3 (Fig. 2), the mole fraction of the oxime present in the path of the measuring spectroscopic beam was estimated as $Q=0.5$. This might have been expected since this corresponds to the change in intensity of the absorption at $270 \mathrm{~m} \mu$ by $45 \%$. The values $\left(\epsilon_{Q}\right)_{270}=20,400$, $\left(\epsilon_{Z}\right)_{270}=2000$, and $\left(\frac{O D_{t}}{O D}\right)_{270}=0.55$ were used to calculate

$Q=0.5$. (The assumption is made that the molar extinction coefficients of both $\alpha$-and $\beta$-p-anisaldoxime, $\epsilon_{\alpha}$ and $\epsilon_{\beta}$ at $270 \mathrm{~m} \mu$ at $-78^{\circ} \mathrm{C}$ are the same as those at $264 \mathrm{mp}$ taken at room temperature).

Equation $I$ is also applied to calculate the value $\left(\frac{O D_{t}}{O D_{0}}\right) 232^{\circ}$. The calculated value for it at $232 \mathrm{~m} \mu$ is $3.8 \mathrm{using}$ the values $\left(\epsilon_{Q}\right)_{232}=2200$ and $\left(\epsilon_{Z}\right)_{232}=13,800$. The observed value is 2.9 at $232 \mathrm{~m} \mu$. The discrepancy will depend largely on the suitable choice of $\left(\epsilon_{Q}\right)_{232}=$ at $232 \mathrm{~m} \mu$. In the above calculation of $\left(\frac{O D_{t}}{O D_{0}}\right)$, the value of $\epsilon_{Q}=\epsilon_{\alpha}$ taken at room temperature, excluding the possible contribution 
of $\epsilon_{B}$, was applied. Since an 1sosbestic point of the $\alpha-$ and B-isomeric oximes does not occur at $232 \mathrm{mu}$, equation 1 will not rigidly apply. A correction, however, is made by considering the relative amounts of the two oxime 1somers which were est1mated to be in the ratio of $1: 1 \%$. Trie calculated value of $\left(\frac{{ }^{2} D_{0}}{2}\right)_{232}$ then diminishes from 3.8 to 3.2 . Due to the uncertainty of the effect of decreasing temperature on the UV spectrum of $\beta$-p-anisaldoxime, the calculated increase in the intensity of absorption at $232 \mathrm{mp}$ is of the right order of magnitude for an intermediate having the UV spectrum of 3-p-methoxyphenyloxaz1rape.

Although an obvious reduction by $45 \%$ in the intensity of absorption at 270 mi 18 observed in curve 3 (F1g. 2), the change in the intensity of the spectrum taken at room temperature (curve 1) at $264 \mathrm{mp}$ is only $12 \%$ in curve 4. However, this is not unexpected in view of the experimental condttions under which the solution of $\propto-p$-anisaldorime was irradiated, namely, by light at $290 \mathrm{mp}$ through optical windows of $1.0 \mathrm{~cm}$ in diameter and at a temperature close to the freezing point of $\mathrm{n}$-hexane $\left(-95^{\circ} \mathrm{C}\right)$. At this temperature, mixing of the solution would be expected to be extremely inefficient. Thorough mixing rould not be expected unt1l higher temperatures are attained.

* TIC analys1s of p-anisaldoximes wh1ch had been 1 rradiated at $77^{\circ} \mathrm{K}$ in a rigld medium appeared to indicate equal amounts of $\propto$ and $\beta$-oximes, based upon visual estimation. 
Thus at the temperature of the experiment, the spectrum observed (curve 3, Fig. 2) could be that of a solution contained in a volume of about $0.8 \mathrm{~cm}^{3}$ as a result of a beam of light traversing through the solution of optical path length of $1.0 \mathrm{~cm}$ and of $1.0 \mathrm{~cm}$ in diameter. If this were the case

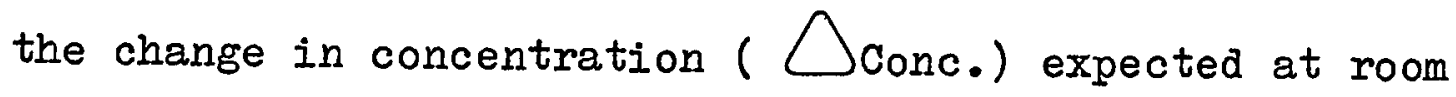
temperature $\left(20^{\circ} \mathrm{C}\right)$ is given by equation 3.

Eq. 3) (Sconc. $)=\frac{1}{2} \times \frac{1}{3} \times c_{0} \approx 1 \times 10^{-5} \mathrm{M}$ where $C_{0}$ is the concentration of $\alpha$-p-anisaldoxime at $20^{\circ} \mathrm{C}$ before irradiation (calculated from the observed optical density and from the previously determined molar extinction coefficient, $\left.\epsilon_{\alpha}=20,4000\right)$. The factor $\frac{1}{2}$ accounts for the amount of p-anisaldoxime relative to the amount of oxazirane, calculated to be in the ratio of $1: 1$ in $0.8 \mathrm{~cm}^{3}$ at $-87^{\circ} \mathrm{C}$. The factor $\frac{1}{3}$ compensates for the decreased volume of $n$-hexane at $-75^{\circ} \mathrm{C}$ estimated from the data of Edujee (112). Therefore, the change in concentration of about $1 \times 10^{-5} \mathrm{M}$ corresponds to a change in the intensity of absorption at $264 \mathrm{~m} \mu$ of $13 \%$, or to the calculated value of $\left(\frac{O D_{t}}{O D_{0}}\right)_{264}=0.88$, (using the value

of $\left(\epsilon_{Q}\right)_{264}=20,400,\left(\epsilon_{Z}\right)_{264}=2000$, and $\left.Q=0.87\right)$ for the reaction mixture when spectra were recorded at room temperature. The observed change in the absorbance was $12 \%$ or $\left(\frac{O D_{t}}{O D_{0}}\right)_{264}=0.88$, in good agreement with the calculated values. 
Ultraviolet spectra presented in Fig.3 show features simllar to those of Fig. 2. A comparison of curve 2 (Fig. 3), which is a spectrum of an unmixed solution at $-70^{\circ} \mathrm{C}$, with curve 3 (Fig. 3), a spectrum of a solution which had been warmed from $-70^{\circ}$ to $+15^{\circ} \mathrm{C}$ and recooled to $-58^{\circ} \mathrm{C}$, shows that the intensity of absorption of the latter solution at $270 \mathrm{~m} \mu$ is larger. This comparison shows that unstirred solutions are not homogeneous, and thus the assumption that the spectrum recorded at $-78^{\circ} \mathrm{C}$ (Fig. 2) is that of a small volume of the irradiated photolyte is probably valid. Fig. 4 illustrates spectra of solutions that have been mixed during exposure to light. It is invariably observed that a maximum appears near $232 \mathrm{~m} \mu$ with a decrease in the absorption maximum at $270 \mathrm{~m} \mu$. The fact that the ratios $\left(\frac{O D_{t}}{O D_{0}}\right) 232$ at both $-68^{\circ}$ and $+20^{\circ} \mathrm{C}$ were about the same $(2.6-2.7)$ is highly suggestive that the intermediate formed is stable for a short period, even at room temperature: P-Methoxyphenyloxazirane, if formed, would not be expected to decompose instantaneously because 3-phenyloxazirane can be prepared in an alkaline aqueous solution from benzaldehyde and hydroxylamine-0-sulfonic acid and isolated as derivatives of benzoyl chloride and phenyl isocyanate $(110 c, d)$.

Aside from securing spectroscopic evidence for the intervention of an oxazirane, one piece of chemical evidence is provided. Although attempts to trap the intermediate from the reaction mixture with phenyl isocyanate were unsuccessful, 
it was demonstrated that the photolyte of $\alpha$-p-anisaldoxime which had been irradiated at $77^{\circ} \mathrm{K}$ in a rigid medium (methylcyclohexane and n-pentane, 4:1 or MCP) with an exciting source at $290 \mathrm{~m} \mu$ contained a substance which possessed an active oxygen. This was shown when a saturated solution of potassium iodide, in isopropanol, was added to the photolyte. The photolyte had been intermittently agitated at $-117^{\circ} \mathrm{C}$ during the exposure. There was an immediate reaction and a dark purple colour, characteristic of iodine developed. This colour persisted at $77^{\circ} \mathrm{K}$ for several hours until the photolyte was warmed to room temperature. The unexposed sample remained colourless on subjection to the same treatment. Furthermore, the results given in Table I show that treatment with potassium iodide reduces the yield of amides by about one half. The difference in the yield indicates that the intermediate being oxidized is responsible for the formation of the amides (see Reaction 26). Therefore, although the test does not conclusively establish the presence of an oxazirane, the evidence so far provided, is consistent with the formation of 3-methoxyphenyloxazirane. Most oxaziranes are known to be highly reactive with reducing agents such as potassium iodide due to the presence of an active oxygen in the three-membered heterocyclic ring. In fact, the reaction of potassium iodide with oxaziranes is the basis of the iodometric procedure for assessing their purity. The reactions have been examined preparatively and form a basis for the reduction of oxaziranes to imines according to 
Reaction $26(110,121)$.

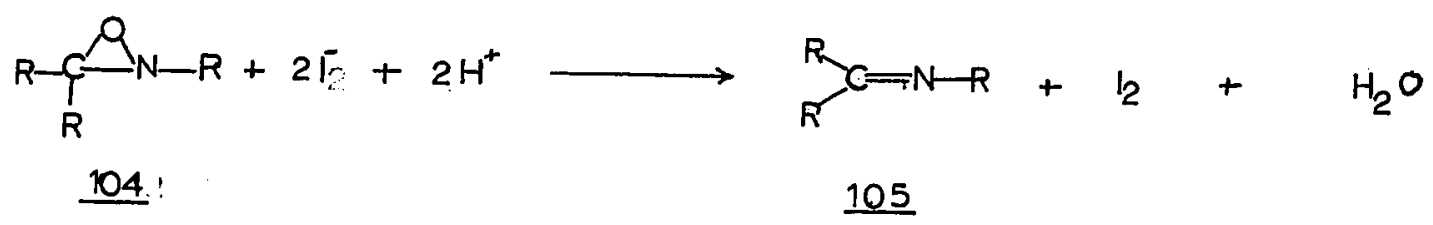

Reaction 26

Attempts to trap the oxazirane with phenyl isocyanate were unsuccessful (see Reaction 27): lthough p-anisamide and $p$-methoxyformanilide were isolated under all experimental conditions. However, one cannot totally exclude the possibility that the originally trapped oxazirane 106 ( $\mathrm{X}=$ $\left.\mathrm{CH}_{3} \mathrm{O}\right)$ formed in small amounts isomerizes to $108\left(\mathrm{X}=\mathrm{CH}_{3} \mathrm{O}\right)$, which is the same product as that obtained from the original starting material. Oxaziranes are sensitive to thermal decomposition and acids. Had the rearrangement of the oxazirane adduct to the urethan occurred spontaneously or on the silicic acid of the TIC plates, one could not detect the "trapped" adduct. The residues from the photolyte were always spotted and eluted at room temperature, and under these conditions transformation of 106 to $108\left(\mathrm{X}=\mathrm{CH}_{3} \mathrm{O}\right)$ might have occurred (see Reaction 27). Schmitz (110c) has reported that reaction $106 \rightarrow \underline{108}(\mathrm{X}=\mathrm{H})$ does occur very smoothly when $106(X=H)$ is warmed in benzene. The urethan $108(X=H)$ is more stable than $106(X=H)$. 


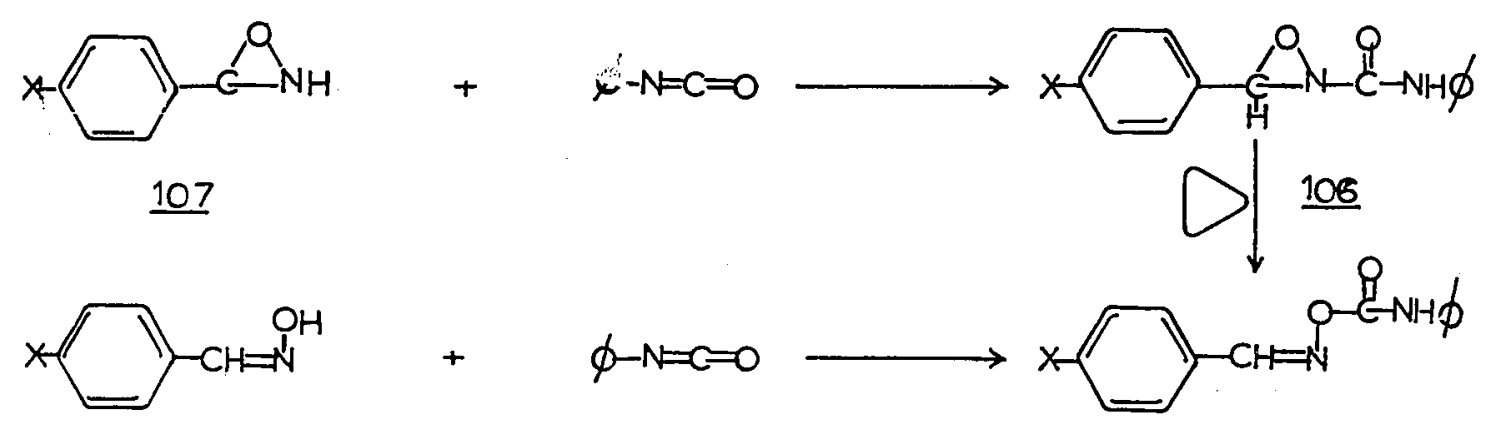

98

108

Reaction 27

If the reaction of potassium iodide with the photolyte of $\alpha-p$-anisaldoxime does, in fact, indicate the presence of 3-p-methoxyphenyloxazirane and reaction occurs according to Reaction 26, since both p-anisamide and p-methoxyformanilide were isolated from the reaction mixture, the amides must be present before potassium iodide was added. The amount isolated could have been formed from the corresponding 3-pmethoxyoxaziranes by a secondary photochemical reaction. Since the 3-phenyloxazirane exhibits a weak absorption band from 250 to $310 \mathrm{mp}$ in the ultraviolet spectrum, absorption of the exciting light at $290 \mathrm{~m} \mu$ can occur. (See Fig. 5). It is pointed out that the photolytes had been warmed to $-117^{\circ} \mathrm{C}$ from $77^{\circ} \mathrm{K}$ periodically to mix the solution in an attempt to renew the concentration of the oximes at the interface of the irradiation ceJl and the rigid solution. Perhaps the interval between mixing was not sufficiently long to prevent depletion 
of the oximes at the interface and, thus, to prevent secondary photochemical reaction of the intermediate. Invariably, under all experimental conditions, irradiation of $\alpha$-p-anisaldoxime at $77^{\circ} \mathrm{K}$ afforded the two amides which were identified by comparing the UV, TLC, and VPC analytical results with those of authentic samples. The results from the treatment of the photolyte with potassium iodide indicated that amides must have been present before the addition of the reducing agent. Moreover, since both the amides are present even after the addition of phenyl isocyanate, the amides must have also been formed before phenyl isocyanate was added to the photolyte. In fluid solutions at higher temperatures the immobility of the solvent and the oxime molecules would be expected to be less critical, especially at room temperature.

Oxaziranes have been reported to be sensitive to light in both rigid and fluid media $(67 \mathrm{~b}, 68,74 \mathrm{~b}, 85 \mathrm{~d}, 113,114)$. Splitter and Calvin (67c) have reported that 2-phenyl-3-pdimethylaminophenyloxazirane appears to rearrange, upon exposure to light in benzene, to 4-dimethylamino-N-phenylbenzamide $(67 \mathrm{c})$. Tanaka and co-workers (68) have found that 2 , 3-diphenyloxazirane was also unstable to light in cyclohexane. In one instance, Padwa (114a) has demonstrated that 2,3-diphenyl-3-benzoyloxazixane can be photochemically transformed to $N, N$-dibenzoylanilide. 
The secondary reaction of $\alpha-p-a n i z a l d o x 1 m e$ due to absorption of light by an oxazirane intermediate, in a rigid matrix, is highly probable in view of the present results and the report that 3-phenyloxazirane can be prepared "In situ" (1100). Furthermore, the fact that irradiation of $\propto-\mathrm{p}-$ anisaldoxime leads to both geometrical and stmuctural 1somerizations at room temperature and at $77^{\circ} \mathrm{K}$, suggests that reactions of the benzaldoximes probably occur by the same processes and are initiated from the same exc1ted state (or states) at all temperatures so far studied.

\section{Maliplicity of States}

The molecularity of the photochemical Beckmann rearrangement being established as intramolecular and evidence for the intermediacy of an oxazirane being provided, quantitative data were then accumulated in order to determine the efficienoy of the reaction and to define the multiplicity of the states responsible for both the structural and geometrical 1somerizations of benzaldoxime.

\section{Suantum Yield Measurement by Sensitization}

Quantum ylelds of benzamide by sensitization were obtained under conditions in which the donor molecules in their triplet states are capable of transferring energy to the acceptor molecules (benzaldoximes) in their ground states. This process was first established by observing the change in the relative amounts of o-benzaldoxime and $\beta$ 


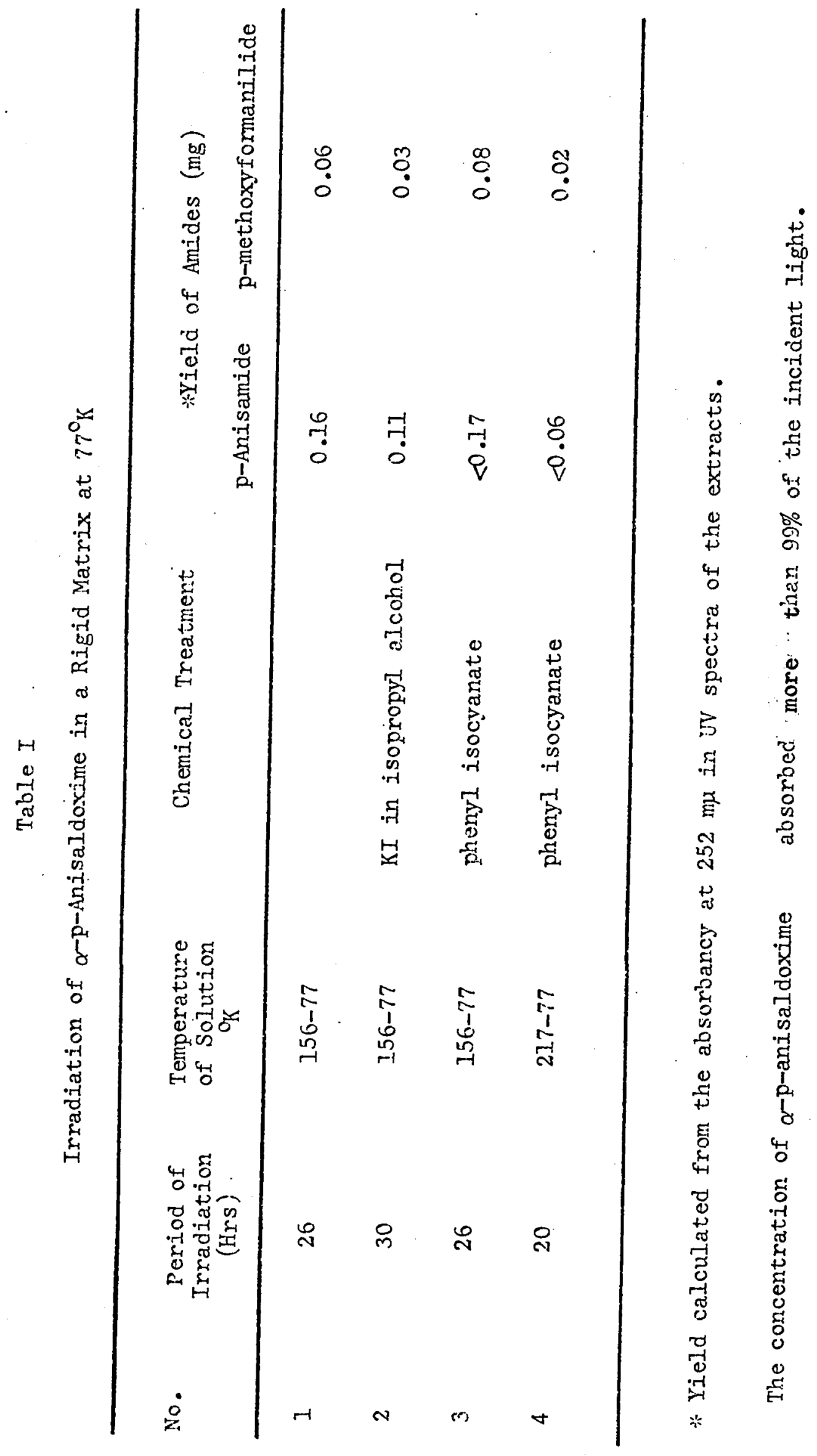




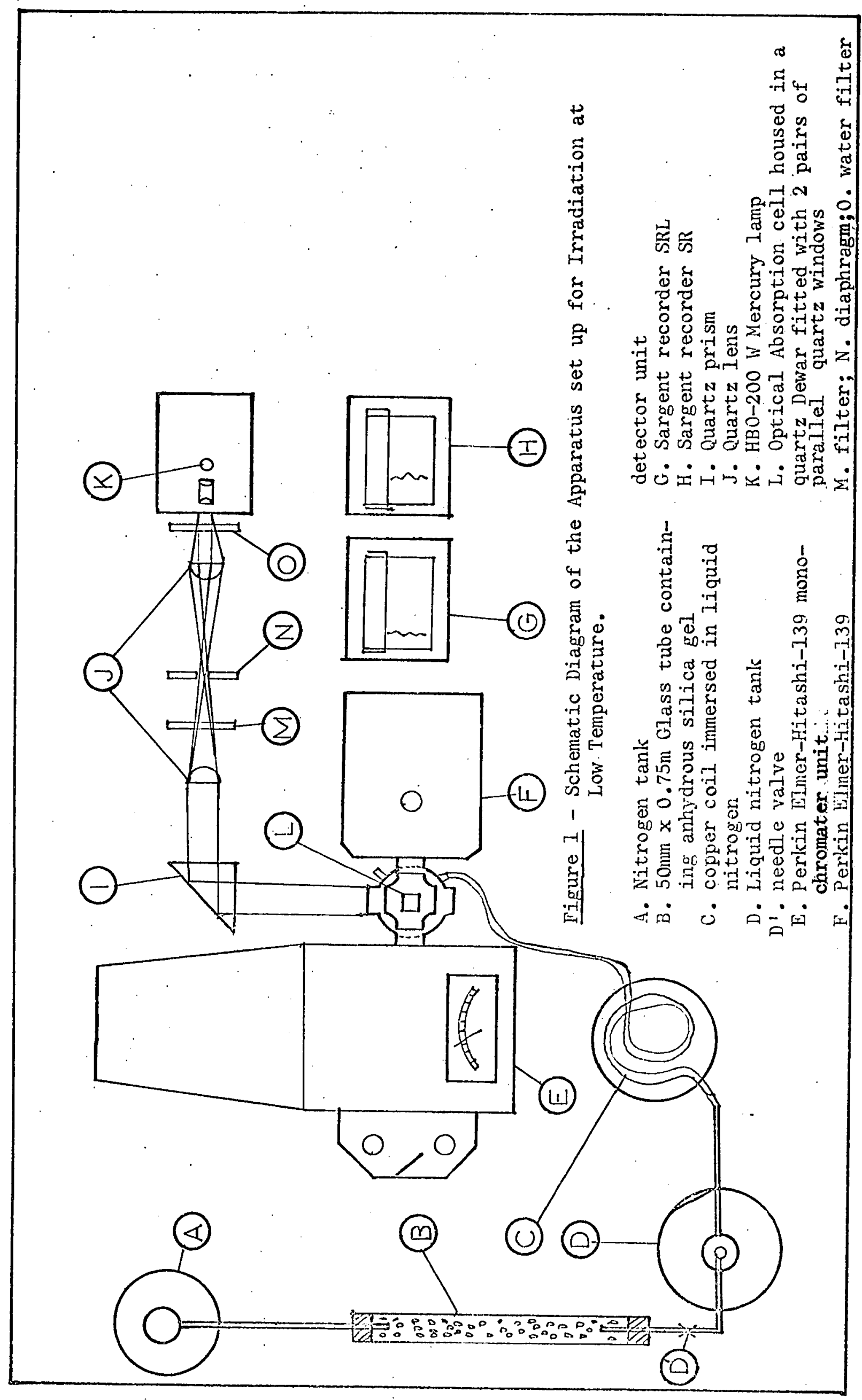


52

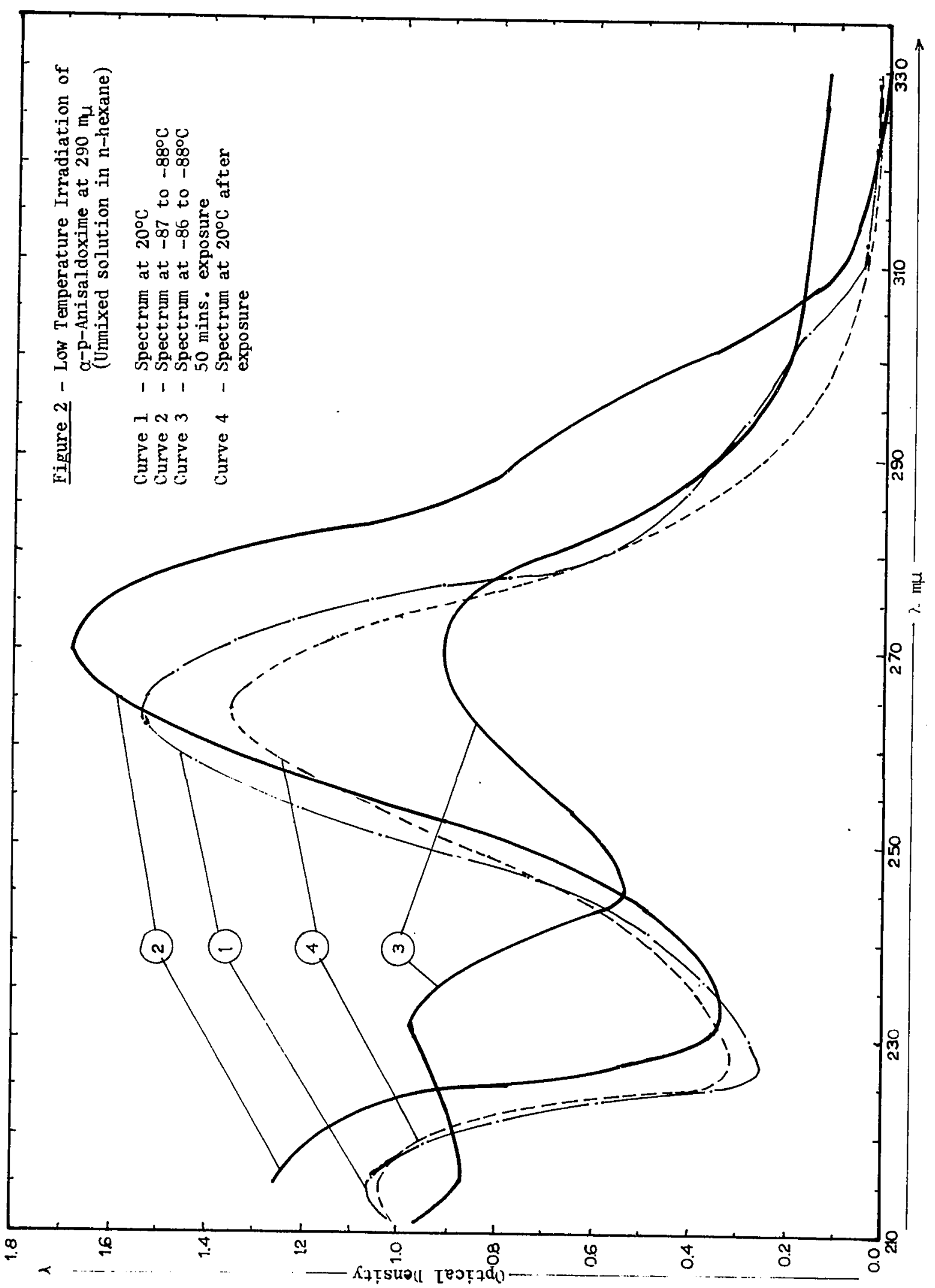




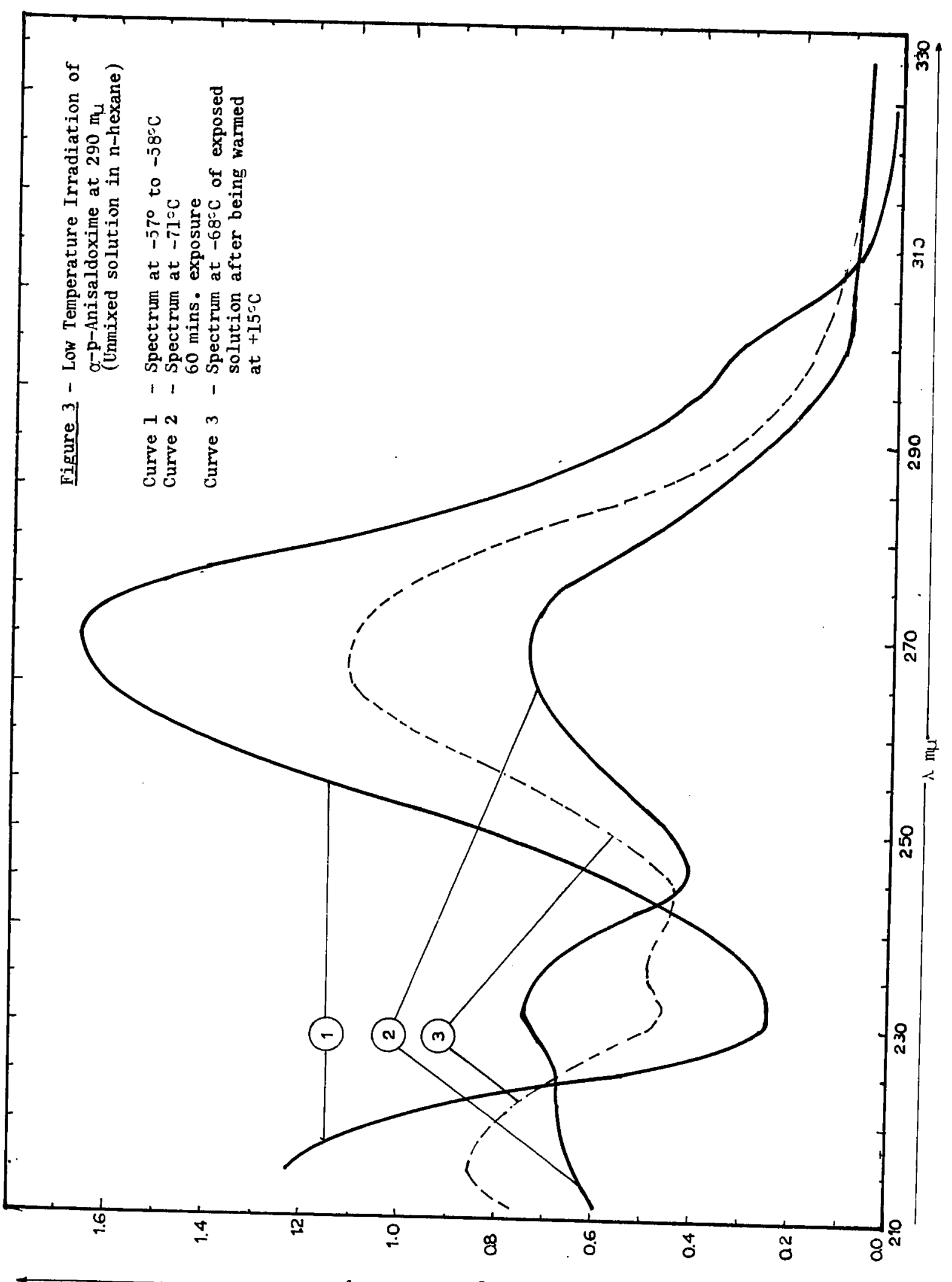




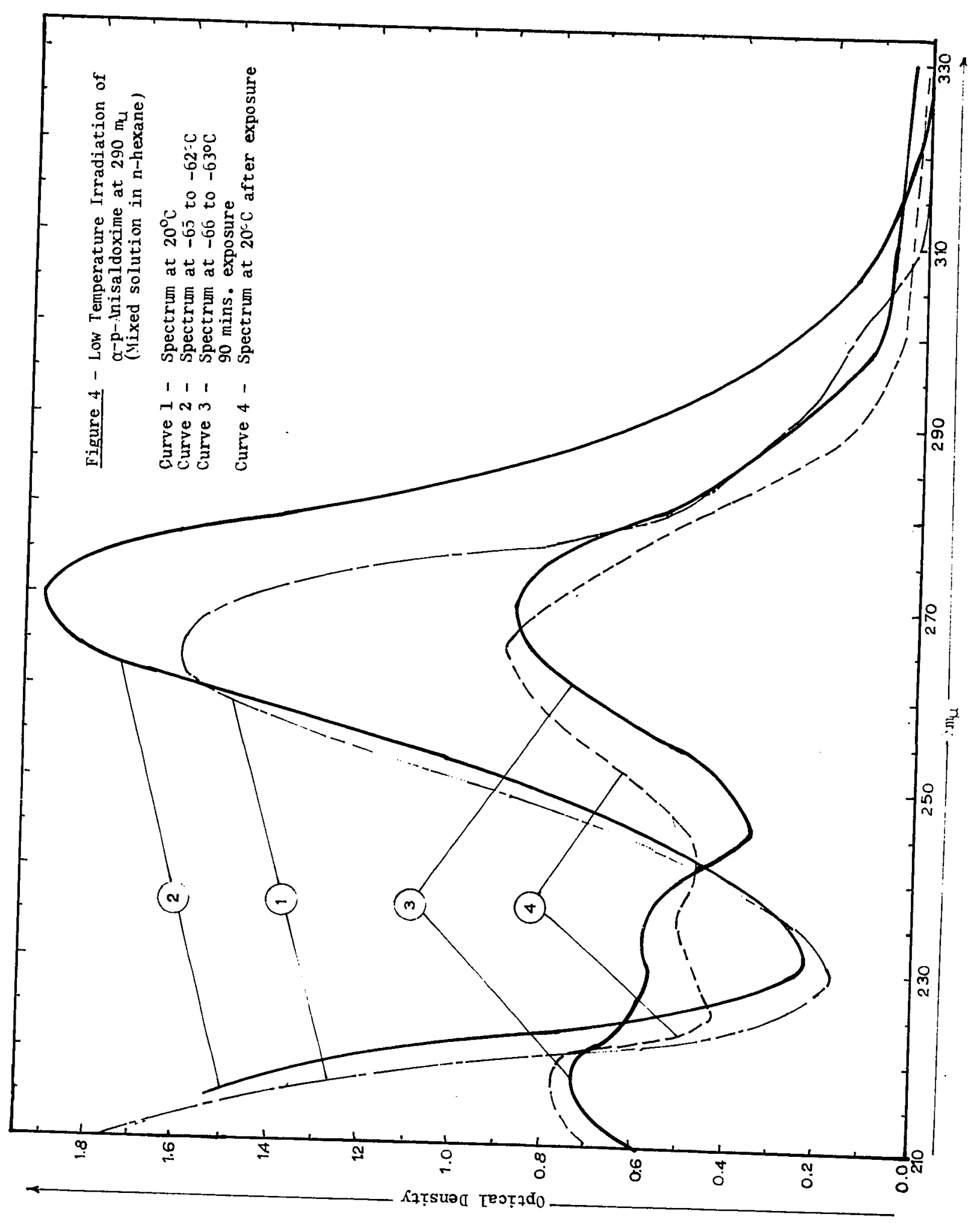




\section{5.}

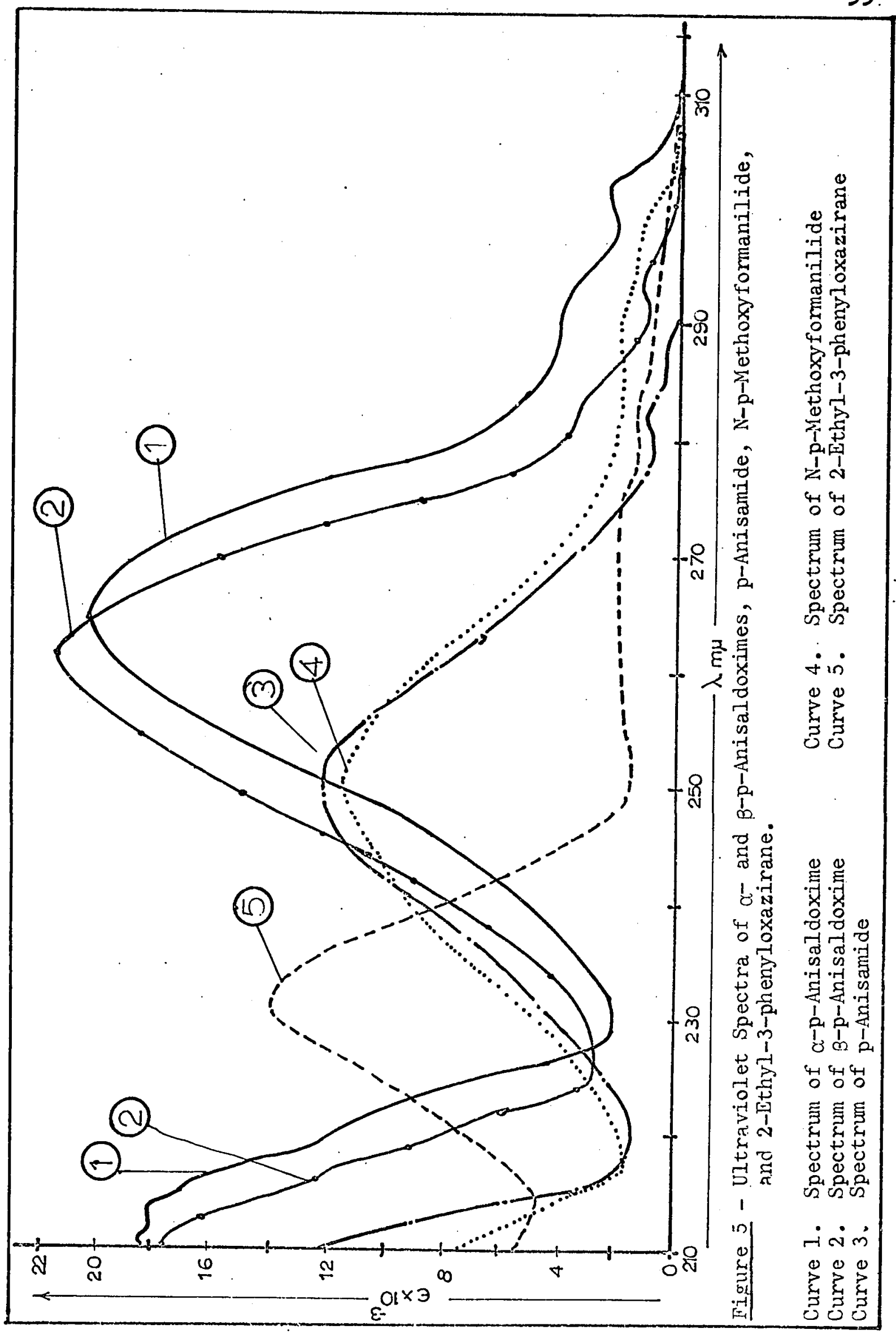


benzaldoxime under the experimental conditions of the investigation. The quantum efficiencies of the rearrangement under aprotic and protonic reaction conditions were compared with the quantum efficiencies of the transformation obtained by direct irradiation of $290 \mathrm{~m} \mu$ under similar conditions. The results obtained by sensitization in the presence and absence of acids are recorded in Table II and those obtained by direct irradiation at $290 \mathrm{~m} \mu$ are given in Table III. The quantum yields of benzamide by sensitization, $\left(\phi_{\mathrm{BZ}-\mathrm{S}}\right) \lambda_{i}$, listed in Table. II are calculated on the assumption that energy transfer from the triplet donor molecules to benzaldoxime is $100 \%$ efficient after allowing for the quantum efficiency of the intersystem crossing ( $\phi_{\text {ISC }}$ ) of the sensitizers. Triphenylene, phenanthrene and acetone* were selected as sensitizers for quantitative measurements. Anthracene was also used but no quantitative results are recorded.

The quantum yields $\left(\phi_{\mathrm{BZ}-\mathrm{S}}\right)_{334}$ by sensitization at $334 \mathrm{~m} \mu$ are less than 0.001 under aprotic conditions. The only photochemical reaction observed is the sensitized geometrical isomerization of $\alpha$-benzaldoxime to $\beta$-benzaldoxime. Absolutely no benzamide was detected by TIC or by spectroscopic methods. In contrast, the quantum yields $\left(\phi_{\mathrm{sZ}}\right)_{290}$ determined by direct irradiation at $290 \mathrm{mp}$ in cyclohexane or dioxane are $\approx 0.002$. Under protonic conditions, for example, in the presence of acetic acid with triphenylene

*I. $0 \mathrm{~cm}$ pathlength of acetone absorbed more than $98 \%$ of the incident light at $290 \mathrm{~mm}$. No benzamide was formed and only a rapid geometrical isomerization 
however, $\left(\phi_{\mathrm{BZ}-\mathrm{S}}\right)_{334}$ is 0.002 . This value is comparable to that obtained by direct irradiation of benzaldoxime in the absence of acetic acid (see Table III). These yields however, are smaller, by a factor of 13, than that determined by direct irradiation at $290 \mathrm{~m} \mu$ under similar reaction conditions.

In the presence of acetic acid, geometrical isomerization by sensitization again occurred. The gradual increase in the concentration of the $\beta$-form was observed as the $\alpha$-isomer was irradiated, using triphenylene as the donor molecule. In the presence of acetic acid and trifluoraotic acid (100:1) the $\alpha$-isomer was highly favoured over the $\beta$-benzaldoxime.

That benzamide was the rearrangement product of photochemical sensitization was confirmed as follows: a) an unexposed solution of triphenylene, benzaldoxime, and acetic acid ( 100 equivalents) was shown to be completely free of benzamide by TIC and IR analysis. b) Irradiation of only benzaldoxime and acetic acid did not afford any benzamide. c) Moreover, illumination of triphenylene and acetic acid alone did not lead to any adduct exhibiting significant absorption in the carbonyl region $\left(1676 \mathrm{~cm}^{-1}\right)$. There is absolutely no interference at this frequency by any of the photolytes serving as control solutions. 
II Quantum Efficiency of Geometrical Isomerization of Benzaldoximes at $290 \mathrm{mp}$

The photochemical efficiency of geometrical isomerization of $\alpha$ - and $\beta$-benzaldoximes were determined at $290 \mathrm{~m} \mu$ at room temperature. The quantum yield of $\beta$-benzaldoxime from the $\alpha$-isomer ( $\phi_{\alpha \beta}$ ) was determined in $n$-hexane, dioxane, and cyclohexane. The value for the reverse process $\left(\phi_{b \alpha}\right)$ was measured in dioxane. The results are recorded in Table IV.

The analytical techniques utilized were the TLC-U.V. spectrophotometry method and the difference method of absorption spectrophotometry. The latter method was used for the determination of $\phi_{\alpha \beta}$ in cyclohexane.

$\phi_{* 3}$ in dioxane at $290 \mathrm{mp}$ was $0.398 \pm 0.013$, slightly larger than the value obtained in n-hexane. Failure to adequately degas the sample did not appear to affect the quantum yield $\phi_{\alpha \beta}$ at all. Instead of degassing the solvent by bubbling a slow stream of nitrogen through the solution before irradiation, oxygen, instead, was bubbled through the solution for 1 hour. The solvent was not treated with alumina* After passage of oxygen for 2 hours, no obvious oxygen-catalyzed geometrical isomerization was observed, and neither were any obvious side products detectable when irradiation had been carried on in dioxane within this period of 2 hours. 
$\phi_{B a}$ determined by only two runs was 0.349+0.024. After correcting for the light absorbed by the $\alpha$-isomer, the quantum yield was 0.38 . The larger error compared to $\phi_{\alpha \beta}$ was mainly due to the $\alpha$-isomer that was present as contaminant. Consequently, a large "blank" correction was required. The sum of the quantum yields $\left(\phi_{\alpha \beta}+\phi_{\beta \alpha}\right)$ was 0.78 in dioxane. The percentage conversion of $\alpha$ to $\beta$ isomer was $6-7 \%$ in $n$-hexane and dioxane and that of the reverse process in dioxane was $5-6 \%$.

The amount of light that would have been absorbed by the $\beta$-form can be estimated from the amount of conversion of $\beta$ - isomer occurring upon irradiation of $\alpha$-benzaldoxime, assuming that the Beer-Iambert Law is applicable at high concentration. Since the total absorbance at $290 \mathrm{~m} \mu$ during exposure will be due to the contribution of both the $\alpha$ - and $\beta$-isomers, the relative amounts absorbed are equal to the ratio of the optical densities of the $\alpha$ - and $\beta$-isomers. The difference in the molar extinction coefficient of $\alpha$ benzaldoxime is greater than that of the $\beta$-isomer by a factor of approximately 2. By taking the average of the final and the initial concentrations of the two isomers as the effective concentration absorbing light during irradiation, only about $2 \%$ of the light would have been absorbed by the $\beta$-form. On the other hand, the absorption of light by the $\alpha$-isomer during irradiation would be expected to be greater. A $7 \%$ conversion of $\beta$ - to $\alpha$-benzaldoxime would correspond to approximately $7 \%$ of the light absorbed by the $\alpha$-isomer. 
Correction for this was made for the $\phi_{\beta \alpha}$ that is quoted here.

II Photostationary States (PSS) or Equilibrium Mixtures by Direct and Sensitized Irradiation

The photostationary states $\left(\frac{\beta_{\infty}}{\alpha_{\infty}}\right)$ which were estimated by direct and sensitized irradiation in dioxane are given in Table $V . \quad\left(\frac{B_{\infty}}{\alpha_{\infty}}\right)_{i}$ was determined by direct irradiation using unfiltered light from a high pressure mercury lamp and by using the $253.7 \mathrm{~m} \mu$ Iine ( $97 \%$ pure) from a low pressure mercury lamp. The photostationary state (PSS) was attained by sensitization with ketonic compounds such as p-hydroxyacetophenone and Michler's ketone, and with aromatic sensitizers such as triphenylene and phenanthrene. Ketonic and aromatic sensitizers were excited at $340 \mathrm{~m} \mu$ and $334 \mathrm{~m} \mu$, respectively. Typical curves exhibiting the establishment of the photostationary state (PSS) conditions are given in Fig. 6 and 7. The analytical technique applied was the TICSpectroscopic method (see experimental). The solvent selected for the experiment was dioxane since both $\alpha$-and $\beta$-benzaldoximes were soluble in it. Preliminary experiments had been conducted in several solvents before dioxane was selected. In neat acetic acid, using benzophenone, no sensitized isomerization was observed. This was probably due to the rapid acid catalyzed reaction which was highly in favour of the $\alpha$-isomer. Comparative phosphorescence measurements on the solution containing benzophenone only and that 
containing benzophenone and acetic acid did not indicate any obvious amount of quenching at $77^{\circ} \mathrm{K}$ in a solvent mixture of ethanol, isopentane, and ethyl ether $(5: 5: 2)$ (EPA). No isomerization was observed in neat acetic acid using benzophenone; since, however, it had been demonstrated that triphenylene and 100 equivalents of acetic acid, could effect isomerization, reservation therefore, must be employed in interpreting any unobserved geometrical isomerization in the presence of large amounts of acetic acid.

Attempts to extend the studies to several sensitizers were, with a few exceptions, unsuccessful. Those suitable for quantitative determination of PSS's were triphenylene, phenanthrene, Michler's ketone, and p-hydroxyacetophenone. For the aromatic hydrocarbon sensitizers and for p-hydroxyacetophenone, the concentrations of the $\alpha$ - and the $\beta$-forms were selected near to those at the PSS's, and then the solutions were irradiated. Ketonic sensitizers generally introduced considerable difficulties into the quantitative determination of the two oxime isomers, since either the sensitizers or the irradiation products interfered with the analyses. Acetophenone, $\alpha$-acetonaphthalene, $\alpha$-naphthaldehyde, benzaldehyde, anthraquinone and benzil all caused geometrical isomerization, but were unsuitable for quantitative determination of $\alpha$ - and $\beta$-benzaldoximes.

Pertinent spectral data of $\alpha$ - and $\beta$-benzaldoximes in dioxane are recorded. The ratios of molar extinction co- 
efficients of $\alpha$ - and $\beta$-isomers $\left(\frac{\epsilon_{\alpha}}{\epsilon_{\beta}}\right)_{i}$ were $1.2,1.2,2.9$, and 1.3 at $253.7,289 \mathrm{~m} \mu, 297$, and $302 \mathrm{~m} \mu$ respectively. The 0-0 energy splittings from the ground states of the first excited singlet states of the $\alpha$ - and $\beta$-isomers were $292 \mathrm{~m} / \mathrm{h}$ $(98.2 \mathrm{k.cal})$ and $290 \mathrm{mp}(99.5 \mathrm{k.cal}$.$) , respectively. The$ difference in the energies of these two isomers was only $1.3 \mathrm{k.cal}$. The relative intensities of the Osram HBO-200 watt mercury $\operatorname{lamp}$ at $289 \mathrm{~m} \mu, 297 \mathrm{~m} \mu$ and $302 \mathrm{~m} \mu$ were $4 \%, 9 \%$ and $13 \%$, respectively.

IV Spectroscopic Evidence for Triplet Energy Transfer from a Sensitizer to Benzaldoximes

The effect of benzaldoxime on the emission spectra of triphenylene and benzophenone at $77^{\circ} \mathrm{K}$ and on biacetyl at room temperature was measured with a phosphorimeter.

$\alpha$-Benzaldoxime in a 5:1 excess over the donor, benzophenone, proved to be a very efficient quencher of the phosphorescence emission. Fig. 8 shows the relative intensities of phosphorescence emitted by benzophenone at $77^{\circ} \mathrm{K}$ and by a solution containing the same concentration of benzophenone and added $\alpha$-benzaldoxime. The comparison clearly indicated that the intensity in the latter instance was considerably reduced. For an $\alpha$-benzaldoxime concentration of $0.83 \mathrm{M}$, or 28-fold excess, emission was completely quenched. The reduction in the blue emission was also observable. A 
similar quenching effect by $\beta$-benzaldoxime on the phosphorescence of triphenylene in the presence of acetic acid was observed (Fig. 9). A slight reduction in the intensity was noticed spectrophotometrically when the concentration of $\beta$-benzaldoxime was $0.014 \mathrm{M}$, an excess of $1.3: 1$. Even when the oxime concentration was $0.07 \mathrm{M}$, a slight reduction in the intensity was observed. Acetic acid had no apparent effect on the phosphorescent emission of triphenylene or benzophenone.

Exactly similar quenching of phosphorescence of biacetyl was observed in a fluid medium at $22^{\circ} \mathrm{C}$. The total quenching of emission was rather impressive in the presence of $0.099 \mathrm{M}$ of $\alpha$-benzaldoxime, an excess $1.6: 1$. The region of recorded emission was from $440.7 \mathrm{~m} \mu$ to $579.1 \mathrm{~m} \mu$ (Fig. 10). At both $77^{\circ} \mathrm{K}$ and $22^{\circ} \mathrm{C}$, the maximum intensity was between 520 and $525 \mathrm{m \mu}$. At $22^{\circ} \mathrm{C}$, the maximum was at $520.3 \mathrm{~m} \mu$ and at $77^{\circ} \mathrm{K}$, it was at $524.0 \mathrm{~m} \mu$, calibrated against mercury emission lines. (The sharp lines shown in all the spectra were calibrating lines emitted from a pen lamp). In the absence of any quencher the spectra of a thoroughly degassed solution were well defined, but in the presence of air or $\alpha$-benzaldoxime, there was an obvious reduction of the intensity at 520.0m effect on the emission spectrum of biacetyl. When air had been introduced into the de-aerated cell, and then degassed again, the phosphorescent emission could be restored. It 
was observed that the intensity of emission diminished as the solution was warmed from $77^{\circ} \mathrm{K}$ to $22^{\circ} \mathrm{C}$.

Attempts to restore the emission spectrum of a solution containing $0.099 \mathrm{M}$ of the $\alpha$-isomer by vigorous degassing were totally unsuccessful. Total quenching of emission occurred five minutes after the solution was warmed up from $77^{\circ} \mathrm{K}$ before room temperature was reached. It is pertinent to note that, only phosphorescence is totally quenched, leaving only residual fluorescent emission at about $490 \mathrm{~m} \mu$. The result of the quenching process was visibly discernible at $22^{\circ} \mathrm{C}$. When oxygen or benzaldoxime had been admitted into a de-aerated solution, the solution became clear, whereas in their absence the solution was cloudy and light yellow.

$\checkmark$ Effect of Oxygen on the Structural and Geometrical Isomerization of Benzaldoxime

In Table IV, and in Table VI, the effects of oxygen on the quantum yields of rearrangement $\left(\phi_{\mathrm{BZ}}\right)_{290}$ and geometrical isomerization $\left(\phi_{\alpha \beta}\right)$ are recorded. oxygen had virtually no effect on $\left(\phi_{\alpha \beta}\right)_{290}\left(\phi_{B Z}\right) 290$ was reduced from 0.025 to 0.008 in the presence of oxygen. All irradiations were conducted at $290 \mathrm{~m} \mu$ in the presence of acetic acid. 


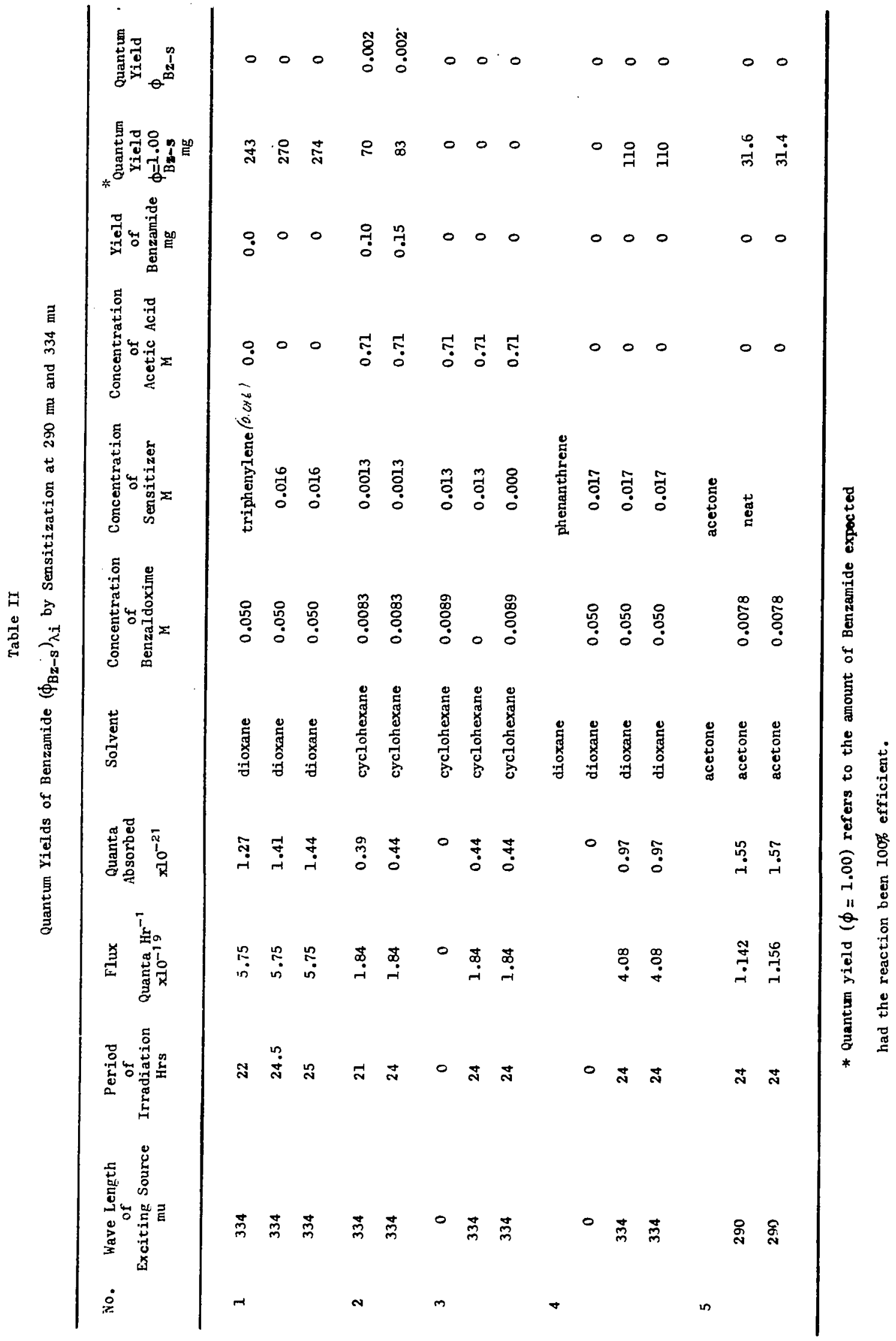


Tablo III

Effect of Wave Lengtha on the Rearrangenent of Benseldoxime

to Benamide in Cyclohexene by Diroct Irradiation

\begin{tabular}{|c|c|c|c|c|c|c|c|c|c|}
\hline No. & $\begin{array}{l}\text { Wave Length } \\
\text { of } \\
\text { Exciting Sourco } \\
\text { m }\end{array}$ & $\begin{array}{c}\text { Period } \\
\text { of } \\
\text { Irradintion } \\
\text { Hrs }\end{array}$ & $\begin{array}{l}\text { Quanta } \\
\text { Absarbed } \\
x 10^{-20}\end{array}$ & $\begin{array}{c}\text { Concentration } \\
\text { of } \\
\text { Benzeldoxdme } \\
M\end{array}$ & $\begin{array}{c}\text { Concentration } \\
\text { of } \\
\text { Acld } \\
M\end{array}$ & $\begin{array}{c}\text { Yield } \\
\text { of } \\
\text { Benzamido } \\
\text { mg }\end{array}$ & $\begin{array}{c}\text { Quantiun } \\
\text { Yiold } \\
\phi_{\mathrm{Bz}=1.00} \\
\mathrm{gig}\end{array}$ & $\begin{array}{c}\text { Quantum } \\
\text { Yleld } \\
\phi_{\mathrm{Bz}}\end{array}$ & $\begin{array}{c}\text { Quantum } \\
\text { Y1eld } \\
\oint_{\mathrm{Bz}} \\
\text { Average } \\
\text { ir }\end{array}$ \\
\hline \multirow[t]{2}{*}{1} & 290 & 21 & 1.69 & 0.0076 & Acetle Aeld & 0.051 & 34.1 & 0.002 & 0.002 \\
\hline & 290 & 21 & 3.18 & 0.0076 & 0.000 & 0.090 & 64.2 & 0.002 & \\
\hline \multirow[t]{2}{*}{2} & 290 & 19 & 3.13 & 0.0071 & 0.0071 & 0.395 & 63.0 & 0.006 & 0.006 \\
\hline & 290 & 29 & 2.92 & 0.0071 & 0.0071 & 0.362 & 58.8 & 0.006 & \\
\hline \multirow[t]{3}{*}{3} & 290 & 20 & 1.12 & 0.0078 & 0.071 & 0.202 & 23.6 & 0.009 & \\
\hline & 290 & 20 & 1.27 & 0.0073 & 0.071 & 0.222 & 25.6 & 0.009 & 0.009 \\
\hline & 290 & 20 & 1.23 & 0.0079 & 0.071 & 0.217 & 24.8 & 0.009 & \\
\hline \multirow[t]{2}{*}{4} & 290 & 21 & 1.22 & 0.0079 & 0.71 & 0.655 & 24.6 & 0.027 & $0.026 \pm 0.001$ \\
\hline & 290 & 16 & 0.90 & 0.0079 & 0.71 & 0.343 & 18.2 & 0.025 & \\
\hline \multirow[t]{2}{*}{5} & 290 & 13 & 1.07 & 0.0073 & 17.3 (neat) & 1.20 & 26.8 & 0.058 & 0.058 \\
\hline & 290 & 12 & 0.87 & 0.0073 & 27.3 (neat) & 1.02 & 17.5 & 0.058 & \\
\hline \multirow[t]{2}{*}{6} & 253.7 & 25 & 0.594 & 0.0071 & 0 & 0.005 & 11.8 & $<0.001$ & $<0.001$ \\
\hline & 253.7 & $25-1 / 2$ & 0.601 & 0.0071 & 0 & 0.005 & 11.9 & $<0.001$ & 0 \\
\hline \multirow[t]{2}{*}{7} & 253.7 & 27 & 0.587 & 0.0071 & 0.0071 & 0.043 & 11.8 & 0.004 & 0.004 \\
\hline & 253.7 & 24 & 0.587 & 0.0071 & 0.0071 & 0.038 & 11.4 & 0.004 & \\
\hline \multirow[t]{3}{*}{8} & 253.7 & 23 & 1.23 & 0.028 & 17.3 (noat) & 0.21 & 24.7 & 0.010 & \\
\hline & 253.7 & 20 & 0.82 & 0.055 & & 0.16 & 16.3 & 0.010 & 0.010 \\
\hline & 253.7 & 20 & 1.18 & 0.055 & & 0.21 & 20.6 & 0.010 & \\
\hline \multirow[t]{3}{*}{9} & 210 & 20 & 1.35 & 0.011 & 0 & 0 & 27.1 & 0 & 0 \\
\hline & 210 & 20 & 1.28 & 0.0070 & 0.0071 & 0.017 & 26.6 & 0.001 & 0.001 \\
\hline & 210 & 20 & 1.23 & 0.0070 & 0.71 & 0.052 & 26.3 & 0.002 & 0.002 \\
\hline \multirow[t]{3}{*}{10} & 290 & & & & $\begin{array}{l}\text { Trifluoroacetic } \\
\text { acid }\end{array}$ & & & & \\
\hline & 290 & 13 & 1.08 & 0.0073 & 0.0071 & 0.750 & 21.8 & 0.035 & $0.034 \pm 0.001$ \\
\hline & 290 & 11 & 1.15 & 0.0073 & & 0.750 & 23.2 & 0.033 & \\
\hline \multirow[t]{3}{*}{11} & 290 & 11 & 1.06 & 0.0073 & 0.071 & 1.02 & 21.2 & 0.045 & \\
\hline & 290 & 11 & 1.19 & 0.0073 & 0.071 & 1.01 & 23.9 & 0.045 & 0.045 \\
\hline & 290 & 11 & 0.93 & 0.0073 & 0.071 & 0.86 & 18.8 & 0.046 & \\
\hline
\end{tabular}

* Errors quoted are standard devlation 


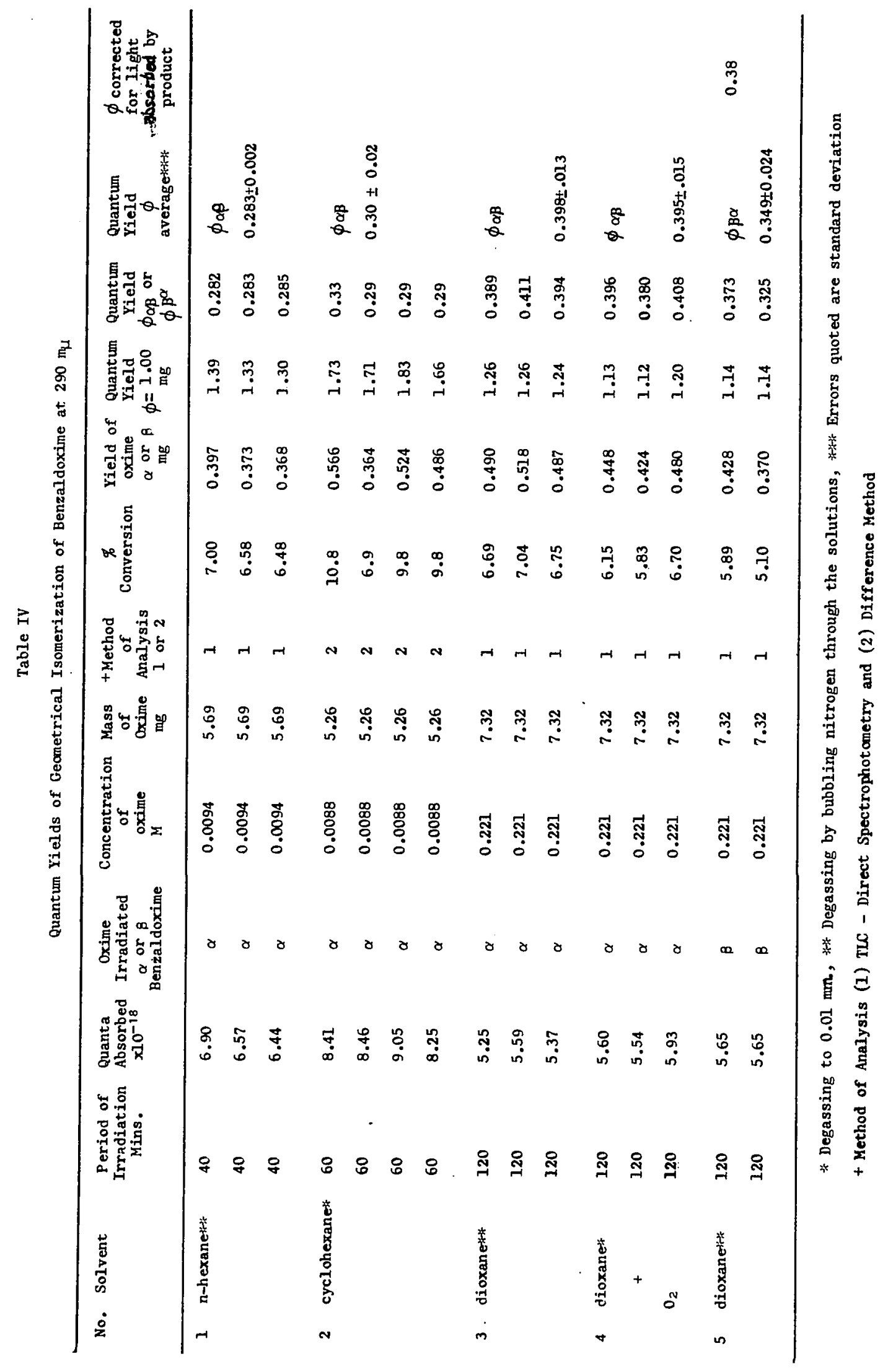




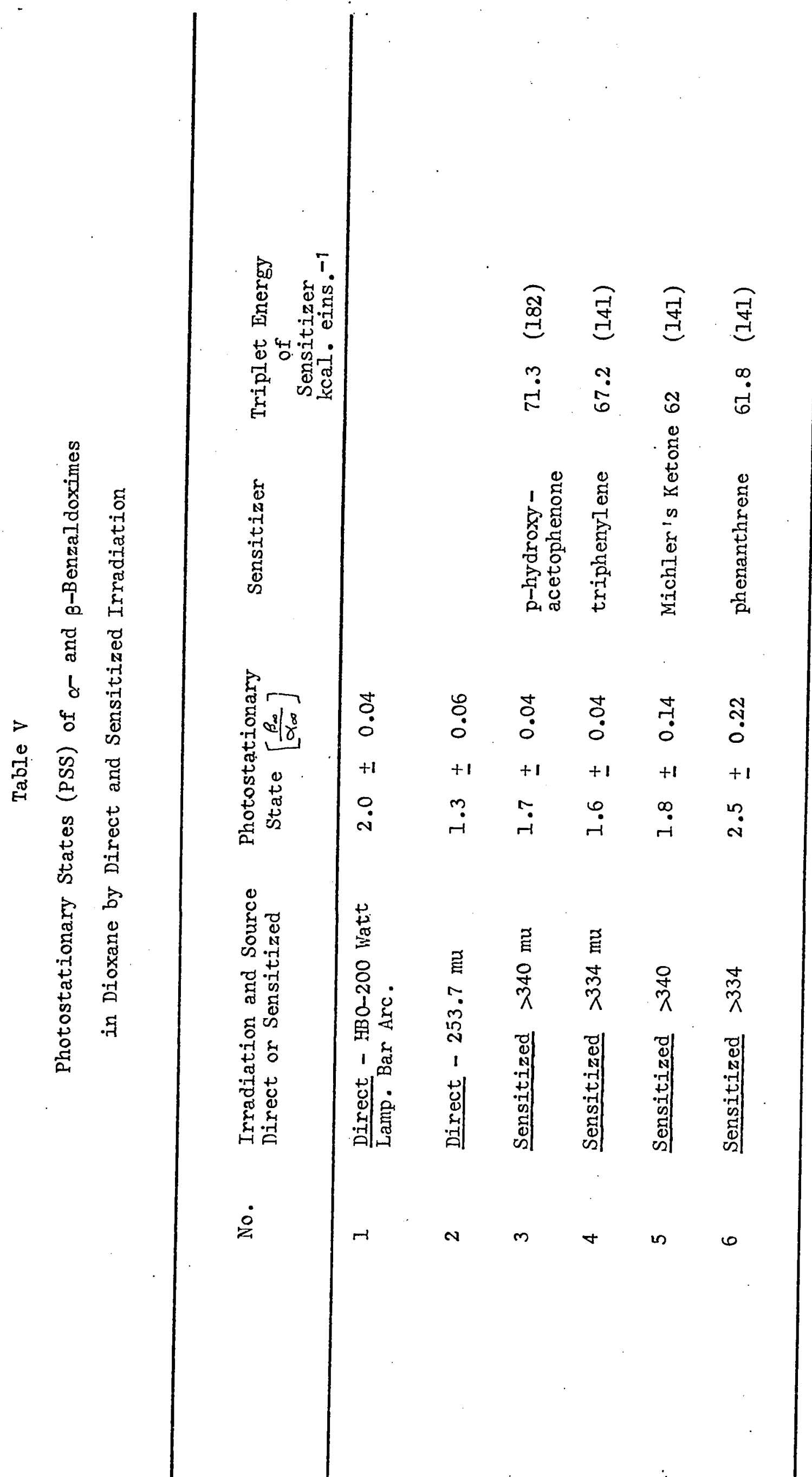




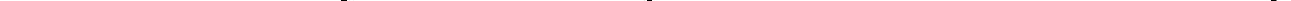




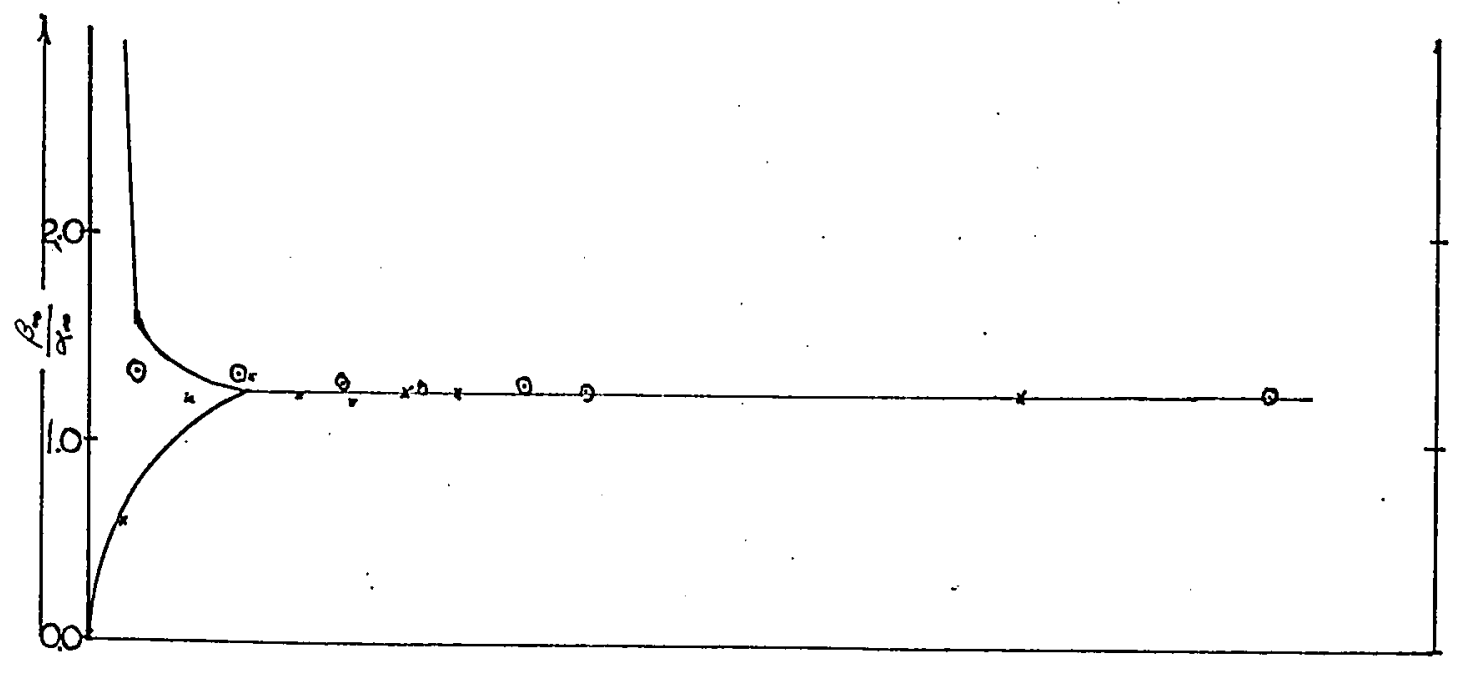

HrS.

Figure 6 - Photostationary State of Benzaldoxime by Direct Irradiation at $253.7 \mathrm{~m}_{\mu}$ in Dioxane.

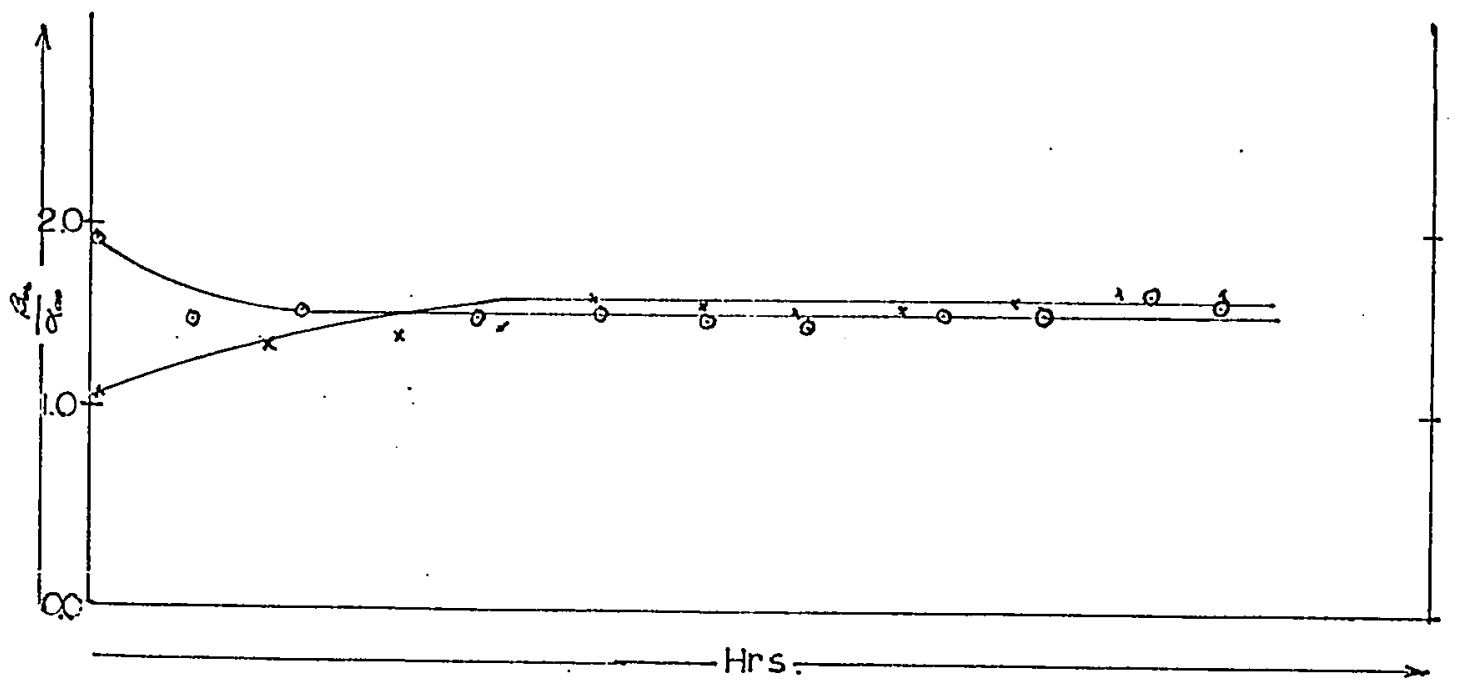

Figure 7 - Photostationary State of Benzaldoxime by Sensitized Irradiation wi.th Triphenylene in Dioxane. 


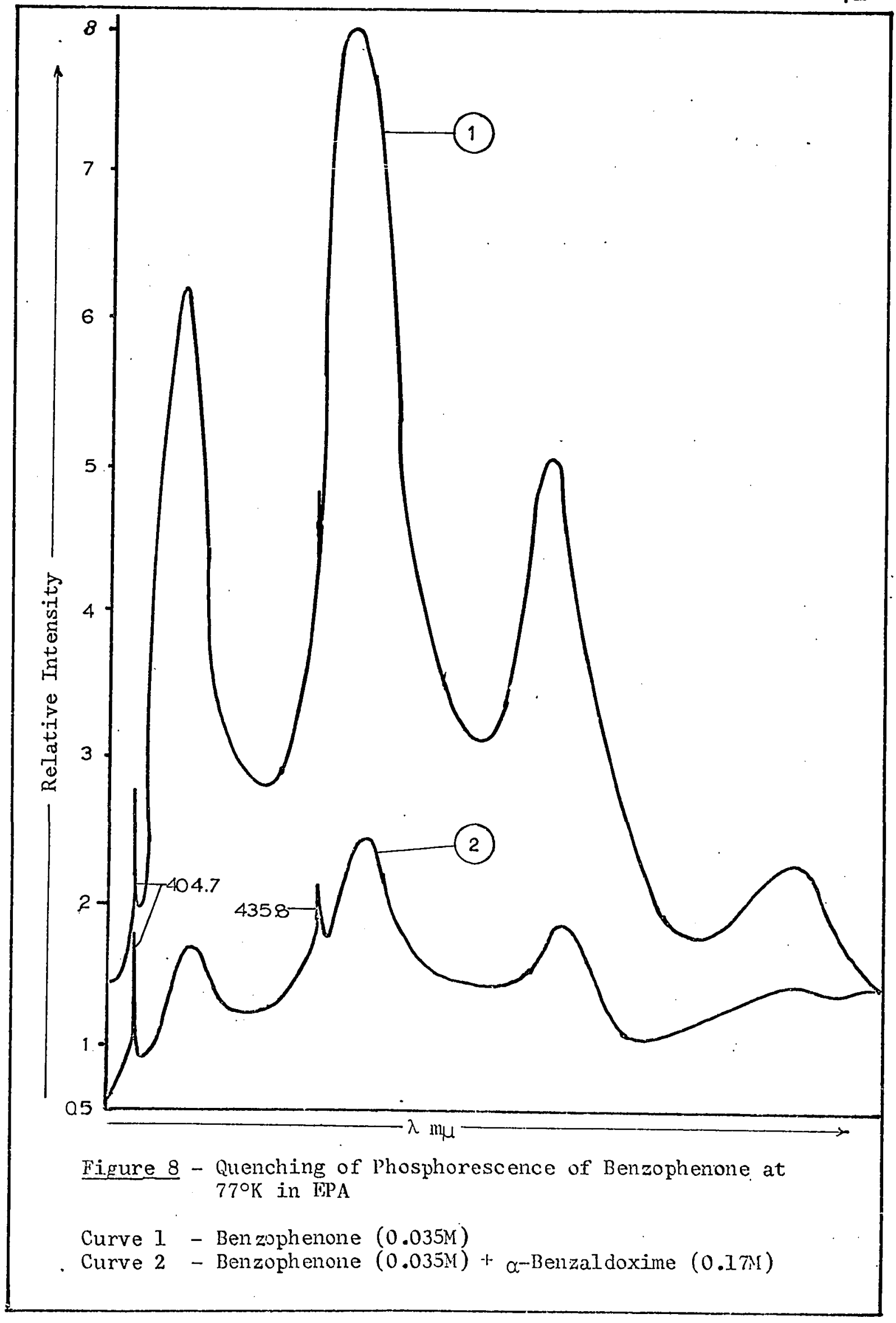


72

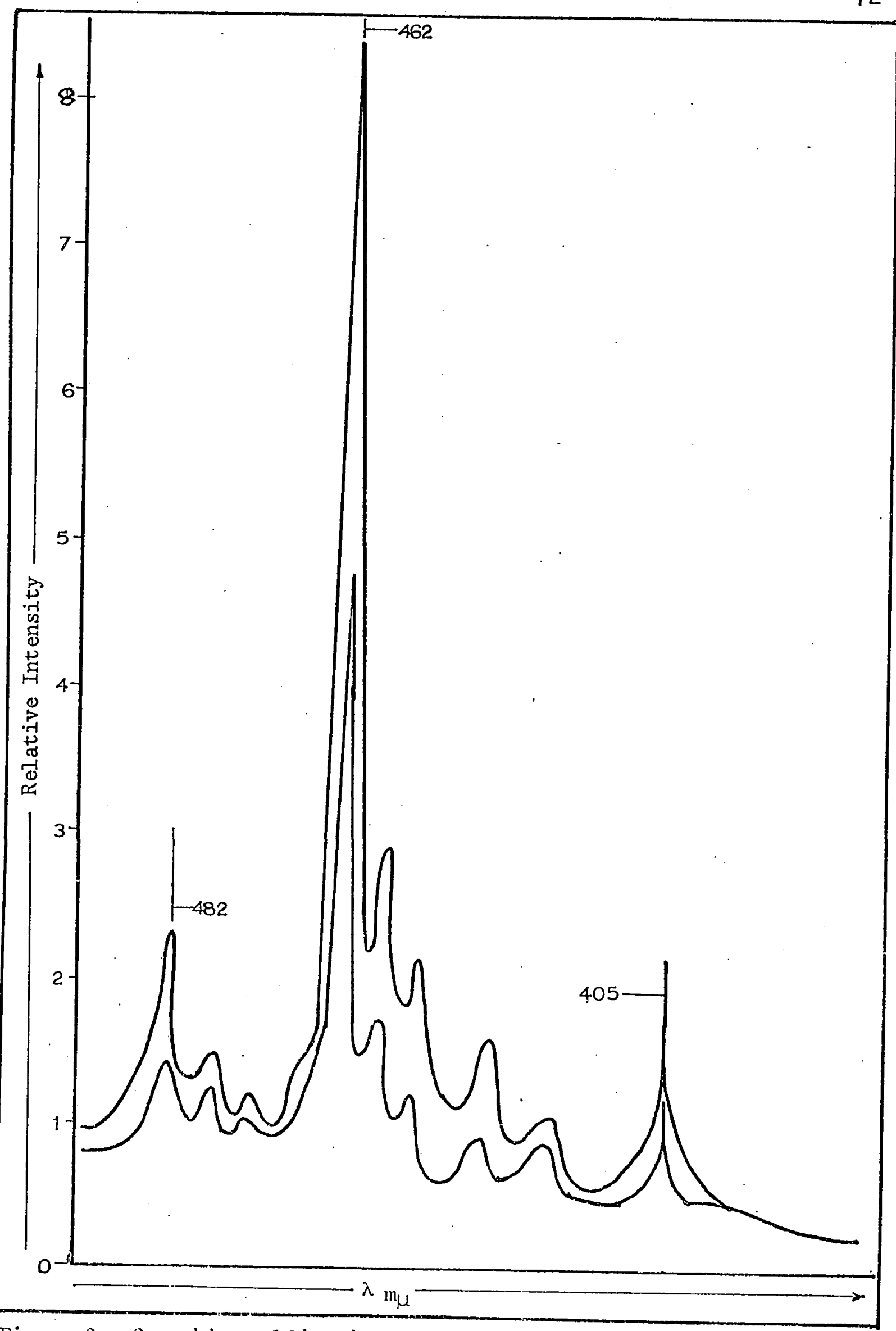

Figure 9 - Quenching of Phosphorescence of Triphenylene at $77^{\circ} \mathrm{K}$ in $\mathrm{BPA}$.

Curve 7 . Triphenylene $(0.071 \mathrm{M})$ in Acetic Acid $(0.0074 \mathrm{M})$.

Curve 2. Triphenyl ene $(0.01 .41)$, Acetic $\lambda$ cid $(0.074 \mathrm{M})$ and 8 -Benzaldoxime 


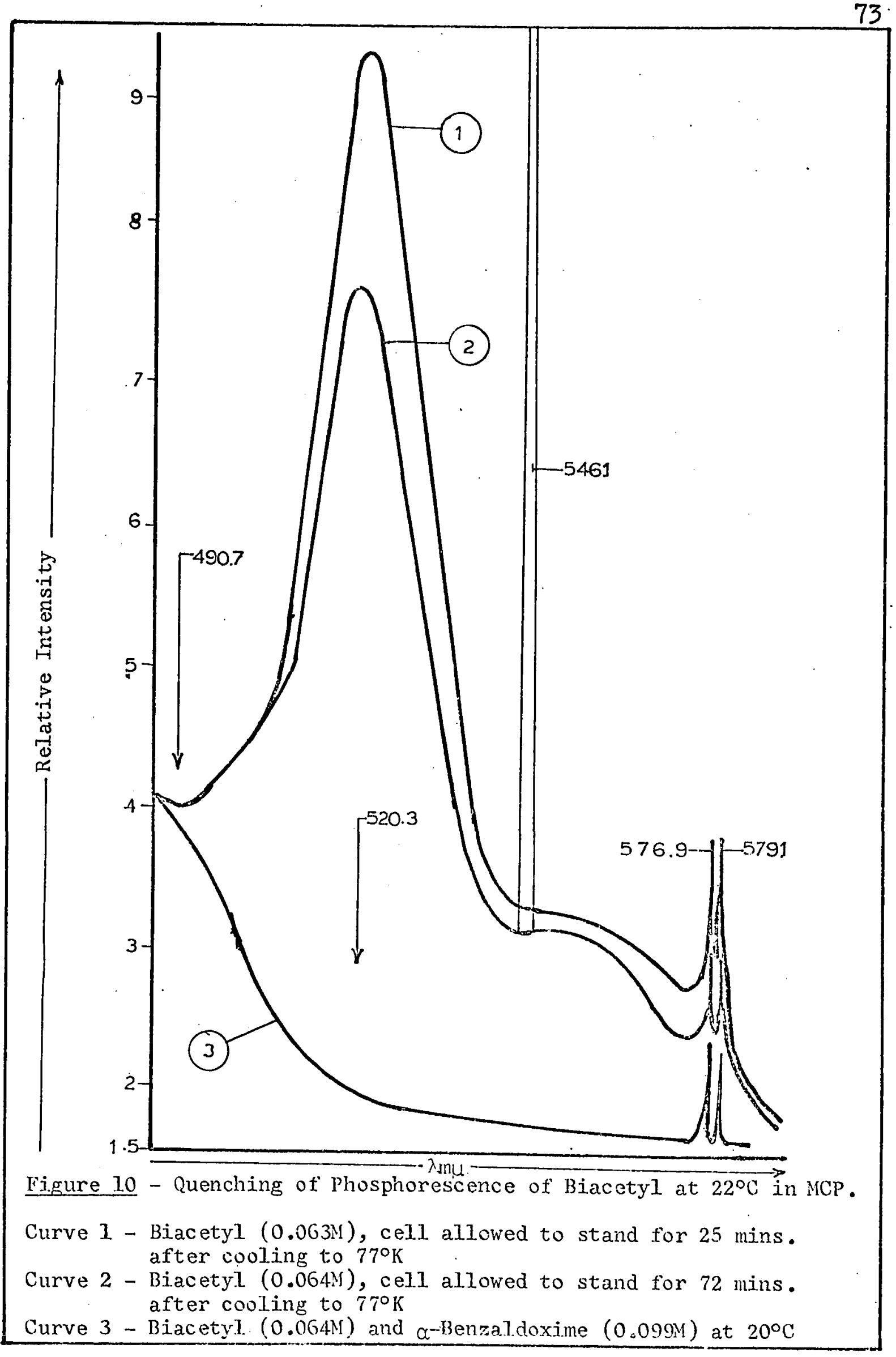


VI Direct and Sensitized Irradiation

The first series of quantum yields for the conversion of benzaldoxime to benzamide had been obtained by direct irradiation, but due to the lack of emission (fluorescence or phosphorescence) from benzaldoxime in a rigid solution at $77^{\circ} \mathrm{K}$ or in a fluid solution at room temperature, no inference on the multiplicity of the states responsible for the reactions of benzaldoxime could be made from these results alone. The only spectroscopic item of data available for benzaldoxime was the absorption spectrum which could offer information about the energy separation between the excited singlet levels and the ground state only. Moreover, since the quantum yield for the rearrangement in neat acetic acid was no greater than $\left|\phi_{B Z}\right|_{290}=0.058$, the fate of ninetyfour percent of the light could not be accounted for from the results of direct irradiations which induce the population of the electronically excited singlet state of the benzaldoxime. The occurrence of other photophysical processes available to the benzaldoxime system could not be excluded to account for the low quantum yield of the rearrangement. Furthermore, the multiplicity of the states associated with both structural and geometrical isomerization required clearer assignment. To define some of the photochemical and photophysical processes inherent in the benzaldoxime system, the rearrangement of benzamide was investigated under conditions in which energy transfer by sensitization could be effected from a suitable donor to an acceptor (benzaldoxime). For many 
organic systems, electronic energy transfer from one organic molecule to another by a collisional triplet energy transfer mechanism is known to be a common mode of energy transfer, a.g. (Eq. 4) (115) where $3_{D}$ and ${ }^{3} A$ denote the donor and the acceptor Eq. $4 \quad 3_{D}+A \longrightarrow D+3_{A}$

molecules, respectivelJ, in their triplet state. This transfer mechanism is a spin-allowed process due to the conservation of total spin angular momentum and has been invoked to explain sensitized phosphorescence of many organic compounds $(115$ - 117). It occurs to us that similar types of energy transfer could be photochemically induced with benzaldoxime originally in its ground state $(A)$, and experiments were designed to demonstrate this. Consideration of the fact that geometrical isomerization of many olefinic compounds has been postulated to occur via a triplet state (118), and in view of the fact that geometrical isomerization is readily induced upon direct irradiation (89, 119 - 122), the existence of a sufficiently low lying triplet accessible by sensitization was deemed highly probable.

A system was selected for optimum conditions for detection of a triplet-triplet energy transfer from benzaldoxime. First, the donor compounds known to have high efficiency of intersystem crossing from an electronically excited singlet to the corresponding triplet state were selected, since this would eliminate the possibility of the 
singlet-singlet transfer by the process:

$$
\text { Eq. } 5 \quad I_{D}+A \longrightarrow D+I_{A}
$$

If this mode of energy transfer occurred to any appreciable extent, the results would not have provided any information about the multiplicity of the states responsible for the different photochemical processes, viz. geometrical isomerization and structural isomerization, since the population of $\mathrm{A}$ by sensitization to a singlet state, ${ }^{1} \mathrm{~A}$, would be equivalent to excitation of benzaldoxime by direct illumination. If the rearrangement did occur from the singlet state ${ }^{1}$, the quantum yield of benzamide would certainly have been expected to be less than that obtained by direct irradiation unless the transfer was $100 \%$ efficient. In view of the short lifetime of the singlet excited state relative to that of the triplet state, complete transfer by process (5) seems unlikely. For aromatic donors such as phenanthrene, anthracene and triphenylene, this process was not observed (115) although singlet-singlet transfers are known to occur with certain systems $(123,124)$. By choosing donors capable of attaining triplet states efficiently, due to the "unforbiddenness" of the direct transition from triplet to ground state, the life of the donor triplet would be expected to be sufficiently longer than that of the singlet for sensitization by the donor in its triplet state to be more probable than that from the singlet state. For some of the ketonic and aromatic triplet donors such as benzophenone, acetophenone, 
acetone, benzil, and triphenylene, the quantum yield of intersystem crossing $\left(\phi_{\text {ISC }}\right.$ ) is known to be greater than $90 \%$. These sensitizers are also known to have a very weak fluorescence intensity or none at all. Another condition which would have optimized the triplet-triplet energy transfer from the donor to benzaldoxime was the selection of a suitable system in which the lowest singlet state of the donor $\left(I_{D}\right)$ lies below that of the acceptor benzaldoxime $\left({ }^{I_{A}}\right)$, so that singlet-singlet energy transfer would be energetically unfavourable. For this phase of the investigation, all the sensitizers met this particular requirement. Therefore, any observed, sensitized reaction, initiated by donor molecules would have been very instructive in deciding whether a triplet-triplet collisional energy transfer had taken place if the donor molecules had a high quantum yield of intersystem crossing and if their lowest excited singlet level was below that of the benzaldoxime. Energies of the first ex- cited singlet $\left(E_{S_{0 \infty}}\right)$ and triplet $\left(E_{\mathrm{T}_{0-0} \ldots}\right)$ states are tabulated in Table VII. It can be seen that values of $E_{\text {So-O }}$ for the donors are well below that of benzaldoxime (98 k.cal.). The highest $E_{\text {So-o }}$ value is that of acetone, which is $13 \mathrm{k} . \mathrm{cal}$. below that of benzaldoxime.

Sensitization of benzaldoxime was monitored by observing the relative change in concentrations of $\alpha$-benzaldoxime and $\beta$-benzaldoxime, since geometrical isomerization proved to be the most efficient photochemical process of benzaldoximes.

* ESo refers to the 0-0 energy separation between the ground state and the excited singlet state. E woo refers to the 0-0 energy separation between the ground state
and the excited triplet state. 
Table VII

Energies of the First Excited Singlet $\left(\mathrm{E}_{\mathrm{SO}-0}\right)$ and the Triplet $\left(\mathrm{E}_{\mathrm{TO}-\mathrm{O}}\right)$ States of Sensitizers

\begin{tabular}{|c|c|c|c|c|c|}
\hline No. & Compounds & $\begin{array}{l}\text { Quantum Efficiency } \\
\text { of Int ersystem Crossing } \\
\varnothing \text { ISC }\end{array}$ & $\begin{array}{c}\text { Singl et Energy }\left(E_{S}\right) \\
\text { keal. eins. }{ }^{-1}\end{array}$ & $\begin{array}{c}\text { Triplet Energy }\left(E_{\mathrm{T}}\right) \\
\text { keal. eins } \text { - }^{-1}\end{array}$ & $\begin{array}{l}E_{T}-E_{S} \\
\text { kcal.eins } \\
-1\end{array}$ \\
\hline \multirow[t]{2}{*}{1} & Benzeldehyde & & $77.4(127)$ & $72(141)$ & $\approx 5$ \\
\hline & & & $98.9(127)$ & & \\
\hline 2 & Benzal doxime & & 98.0 & & $36-49$ \\
\hline 3 & Styrene & & $98.3(149)$ & $61.7(125)$ & $\approx 36$ \\
\hline \multirow[t]{2}{*}{4} & Biacetyl & & $65.6(142)$ & $57 \quad(143)$ & $\approx 6-11$ \\
\hline & & & $62.1(115)$ & $56.3(115)$ & \\
\hline \multirow[t]{2}{*}{5} & Acetone & $1.00(140)$ & $79.5(144)$ & $>70 \quad(143)$ & $\approx 15$ \\
\hline & & $0.40(139)$ & $86.8-87 \quad(138)$ & $\approx 72 \quad(140)$ & $\approx 15$ \\
\hline \multirow[t]{3}{*}{6} & Triphenyl ene & $0.95(126)$ & $80.6(115)$ & $67.4(115)$ & $\approx 13$ \\
\hline & & & $83.3(141)$ & $67.2(141)$ & \\
\hline & & & $83.4(174)$ & & \\
\hline \multirow[t]{3}{*}{7} & Phenanthrene & $0.67(126)$ & $82.9(115)$ & $61.8(115)$ & \\
\hline & & & $81.0(144)$ & & $\approx 20$ \\
\hline & & & $82.8(198)$ & & \\
\hline 8 & inthracene & & 74.5 (115) & $42 \quad$ (115) & $=-30$ \\
\hline 9 & Benzophenone & $1.00(126)$ & $79.5(115)$ & $69.2(14 I)$ & $\approx 10$ \\
\hline
\end{tabular}


That the reaction was entirely induced by sensitization under the experimental conditions was established by observing the absence of any reaction when benzaldoxime alone was irradiated with filtered light above $322 \mathrm{~m} \mu$. In the presence of a sensitizer, geometrical isomerization invariably occurred. These results demonstrated the effectiveness of the filtering system and that the excitation of benzaldoxime by direct singlet to singlet absorption of a low lying ${ }^{l}$ ( $n *$ ) electronic level above $322 \mathrm{~m} \mu$ or by direct singlet to triplet $3\left(n x^{*}\right)$ level is highly improbable, had such an electronic transition state existed in the benzaldoxime.*

When benzaldoxime was sensitized under aprotic conditions, only the geometrical isomerization was unaccompanied by the formation of benzamide. Since most of the donors had high $\left(\phi_{\text {ISC }}\right)$ values and since $E_{\text {So-O }}$ of the donors are below that of benzaldoxime, the geometrical isomerization which was observed must have been a consequence of the triplet-triplet energy transfer from the donors to berizaldoxime.

In the U.V. spectrum of $\alpha$-benzaldoxime in cyclohexane, the intense vibronic peak at $290.5 \mathrm{~m} \mu(98.0 \mathrm{k}$.cal.) was assigned as the $0-0$ singlet separation ( $E_{\text {So-0 }}$ ) by analogy with that of styrene at 291.5mp. The spectra of styrene and benzaldoxime can be superimposed : and appear to undergo transitions of the same type. The origin of the singlet band occurs at about $310 \mathrm{~m} \mu(92 \mathrm{k} . \mathrm{cal}$.$) . If the analogy of the$

*See the section under Effectsof Wavelength 
absorption spectra of styrene could be equally extended to the energy level of the triplet state in benzaldoxime, then the $3\left(\pi \pi^{*}\right)$ transition would correspond to about 60-62 k.cal. $\mathrm{E}_{\text {TO-O }}$ of styrene has been determined by Evans (125) as 61.7 k.cal. and for $\beta$-methylstyrene, as $59.8 \mathrm{k} . \mathrm{cal}$. by Hammond (116). It is, however, recognized that no rigorous exclusion of a low lying ${ }^{l}\left(n x^{*}\right)$ transition band and a highly forbidden $3\left(n \pi^{*}\right)$ band due to participation of the non-bonding electrons in the $\mathrm{C}=\mathrm{N}$ chromophore, has been made. In a highly concentrated solution, however, no band above $310 \mathrm{~m} \mu$ was observed for $\alpha$-benzaldoxime, thus indicating that these low lying ${ }^{1}\left(n \pi^{*}\right)$ and ${ }^{3}\left(n \pi^{*}\right)$ bands are probably absent. In benzaldehyde, from which the benzaldoxime had been prepared, the ${ }^{I}\left(n \pi^{*}\right)$ transition extends from 300-380m $\mu$ with first singlet $0-0$ of $I\left(n \pi^{*}\right)$ band of absorption spectrum at $370 \mathrm{~m} \mu$ (76.4 k.cal.) (137) and a "forbidden" singlet-triplet ${ }^{3}\left(n \pi^{*}\right)$ excitation band (molar extinction of $=0.1$ ) appears at $397 \mathrm{~m} \mu$ (72 k.cal.) (128), coincident with the 0-0 phosphorescence band of benzaldehyde. Had there been any low lying ${ }^{l}\left(n \pi^{*}\right)$ transition in the ultra-violet spectrum of benzaldoxime, comparable to that observed in the region for benzaldehyde, one might have expected to observe some absorption. One can only conclude that the transition probability in this region of benzaldoxime must be extremely low. The $C=N$ chromophore of aliphatic oximes, like that of ethylene, appear in the vacuum ultraviolet region $(185-190 \mathrm{~m} \mu)$, and no absorption maximum above $300 \mathrm{~m} \mu$ has been reported. The absence of a low Iying $I\left(n \pi^{i}\right)$ 
absorption in the ultra-violet spectrum does not imply, however, that there is also an absence of the corresponding $3\left(m x^{*}\right)$ triplet levei. In view of the lack of phosphorescence from benzaldoxime, the existence of the $3\left(n \pi^{*}\right)$ triplet level cannot be totally excluded. Therefore, if a corresponding ${ }^{3}\left(\pi \pi^{+}\right)$level of the same order of magnitude as that observed in styrene also occurs in the benzaldoxime, a lower lying ${ }^{3}\left(n \pi^{*}\right)$ band might also be present. In 1964, Saito (130) and co-workers reported that, in the absorption spectrum of cyclohexanone oxime, a ${ }^{l}\left(x \pi^{\prime}\right)$ band appeared at $192 m \mu(\epsilon=$ $6,900)$ and $a^{1}\left(n \pi^{*}\right)$ band appeared at $310 \mathrm{~m} \mu(\epsilon=15)$. However, in the spectrum of the same compound reported by Huisgen (129), inspection of the spectrum shows a cut-off appears at about $260 \mathrm{~m} \mu$. Koyano and Tanaka (69) have reported that geometrical isomerization about the $C=\mathbb{N}$ bond of

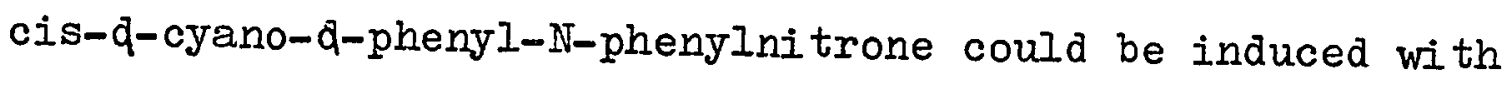
tetracene which has a triplet energy as low as $28 \mathrm{k} . c a l . *$. The last compound is analogous to the benzaldoxime system.

Since no phosphorescence had been detected from benzaldoxime, there is no direct evidence for the population of the triplet state. However, spectroscopic evidence for the direct triplet energy transfer from the donor to benzaldoxime was clearly demonstrated by measuring the decrease in the intensity of the phosphorescence of the donor in the presence of the oxime. Since it is now well established that phosphorescence is directly related to the population

* The quantum yield of sensitization was not reported. 
of a triplet state of an emitting source (131), any observed reduction of phosphorescence of a donor by an acceptor molecule must mean that the triplet states of the former are being quenched. In order that no spin conservation laws are violated, the transfer must occur by a triplet-triplet energy transfer (115). In a rigid solution at $77^{\circ} \mathrm{K}$, phosphorescent emissions of benzophenone and triphenylene were quenched in the presence of benzaldoxime. In a large excess of benzaldoxime, phosphorescence was totally quenched. In a fluid solution at room temperature, when the concentration of benzaldoxime exceeded $\therefore . .$. : that of biacetyl by only a factor of 1.5, total quenching was also observed. However, in a rigid solution at $77^{\circ} \mathrm{K}$, or near the melting point of EPA, phosphorescence could still be observed from biacetyl. Total quenching was observed by the time the solution had warmed up to room temperature. These results certainly indicate the importance of a diffusion contrated process in which the medium allows greater mobility of the interacting molecules and also suggest that triplet-triplet transfer is of a collisional nature.

Numexous cases of triplet-triplet energy transfer proceeding by a collisional process have appeared in the literature, and from these studies it has been shown that this mode of energy transfer generally leads to suppression of the phosphorescence of biacetyl and sensitization of the acceptor. In solutions of biacetyl, phosphorescence is 
observed to be readily quenched by acceptors having lower triplet states than that of biacetyl (117,132). Where the triplet energy ( $E_{\mathbb{T} O-0}^{D}$ difference between biacetyl acting as a donor, is in excess of the triplet energy $\left(\mathrm{E}_{\mathrm{T}_{\mathrm{O}-0}}^{\mathrm{A}}\right)$ of the acceptor by approximately $1000 \mathrm{~cm}^{-1}$ or $3 \mathrm{k} . c a l$. , the energy transfer, due to the exothermicity of the reaction, is collisional, and the rate is very close to that of a

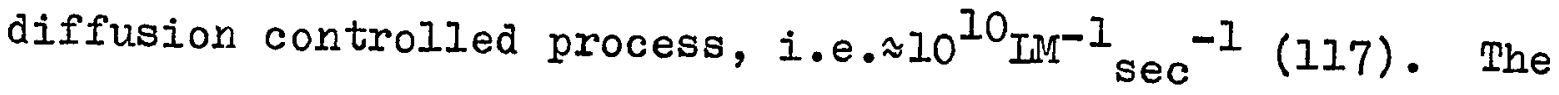
biacetyl triplet energy $\left(E_{\text {TO-O }}\right.$ ) is about $55-57 \mathrm{k} . c a l$. (see Table VII), which means that any quencher molecules with triplet levels below $52-54 \mathrm{k}$.cal. would be susceptible to the same type of collisional triplet-triplet energy transfer. In view of the fact that the biacetyl triplet is quenched by anthracene $\left(E_{\text {TO-O }}\right)=43 \mathrm{k}$.cal., at the rate of $0.81 \mathrm{x}$ $10^{10} \mathrm{IM}^{-1}$ sec very close to the maximum diffusional control rate $\left(I \times 10^{10_{\mathrm{IM}}-1} \mathrm{sec}\right)(116,117)$, and in view of the fact that sensitization of benzaldoxime can be readily effected by anthracene, and further that singlet-singlet energy transfer from biacetyl to benzaldoxime is energetically unfavourable (separation of singlet levels is about $33 \mathrm{k} . c a l$.$) , triplet-$ triplet energy transfer from biacetyl to benzaldoxime probably occurs at a similar diffusional controlled rate. Moreover, although no exact value of the $E_{T}$ level of benzaldoxime can be assigned, it might very well be as low as $43 \mathrm{k}$. cal. and probably well below that of biacetyl ( $E_{\mathrm{T}}=54-57 \mathrm{k}$. cal.). It has been extensively documented that, as the energy separation between the lowest triplet levels of the 
donor and acceptor becomes smaller, the electronic energy transfer by a triplet-triplet transfer becomes much I?ss efficient and considerably more complicated before transfer ceases $(117,118)$. As the triplet energies become comparable, the transfer probability is reduced and no quenching is observed by acceptor molecules with triplet levels higher than that of the donor (123). Backstom and Sandros (117), have suggested that, instead, reverse process can also occur as energy separation becomes small.

Referring to Fig. Il for the case of biacetyl, total emission of both phosphorescence and fluorescence was measured. In the presence of benzaldoxime, only phosphorescence emission is totally quenched and this quenching is shown by the decrease of the intense emission peak at $520 \mathrm{m \mu}$. However, a residual emission between 520-490m $\mu$ remains. Since the emission spectrum of the "blank" consisting of the cell and the solvent did not show this same emission peak, and since no fluorescence or phosphorescence emission occurs from benzaldoxime, the residual emission band which remained in the presence of benzaldoxime must have been due to fluorescence which occurs as radiant emission from the singlet state of the biacetyl (133). The finding is highly significant since it means that the energy transfer from biacetyl to benzaldoxime via singlet-singlet energy transfer by the process represented by (Eq. 5) did not occur to an appreciable extent. The assignment of the 0-0 Ist excited 


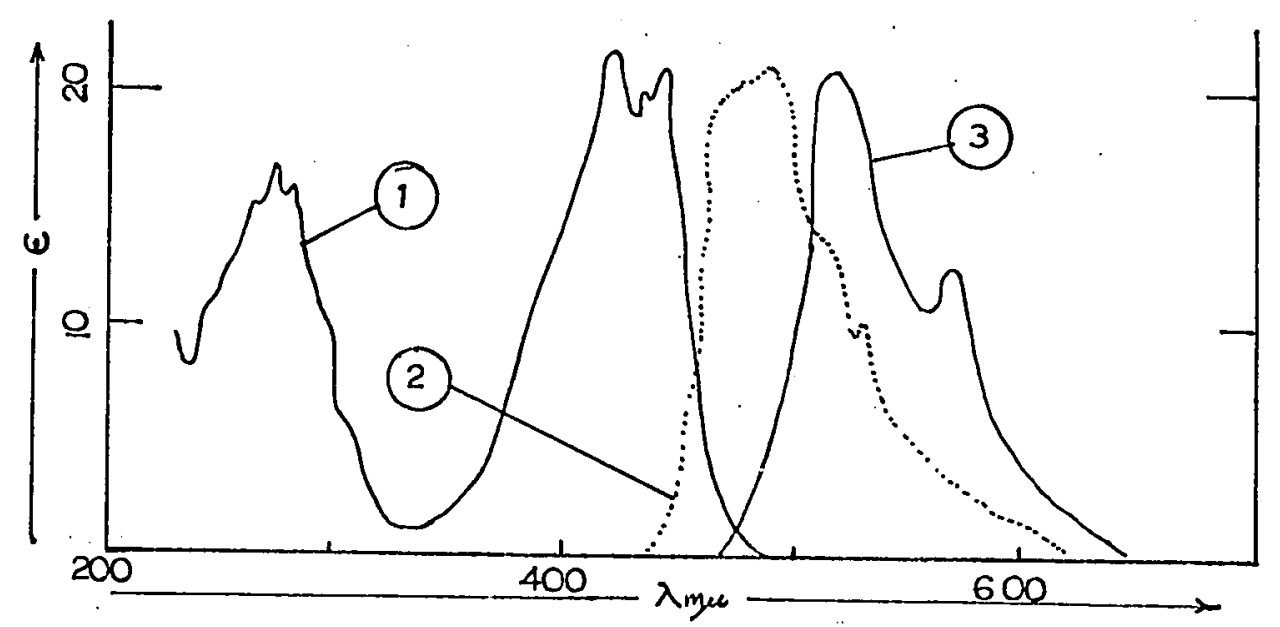

Figure 11 - Absorption and Emission Spectra of Biacetyl in Cyclohexane.

Curve 1 - Absorption spectrum (ref. 124, p. 115)

Curve 2 - Fluorescence spectrum (ref. 124, p. 115)

Curve 3 - Phosphorescence spectrum (117c)

Curves 2 and 3 are spectra showing relative intensity measurements.

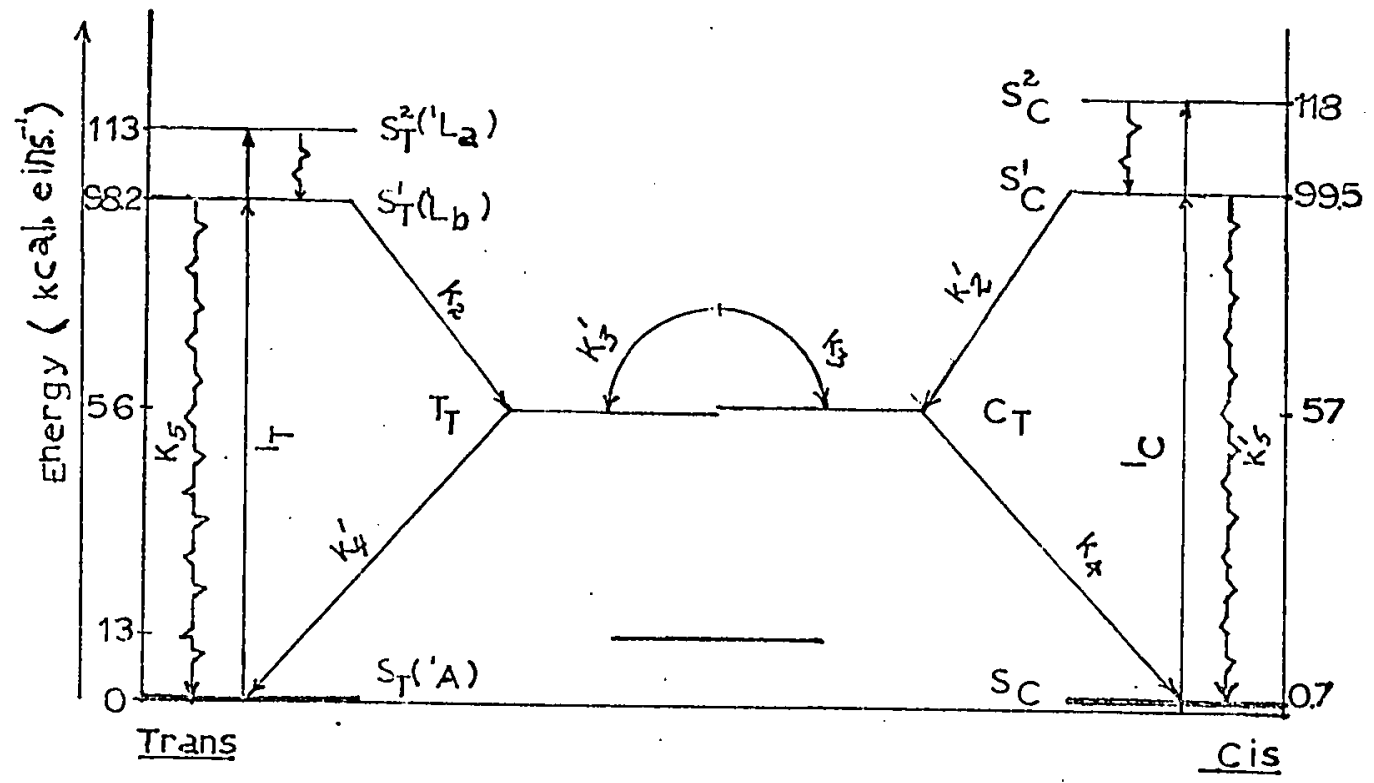

Figure 12 - Potential Tnergy Diagram of $\alpha$-Benzaldoxime and $\beta$-Benzaldoxime in nioxane. 
singlet level of benzaldoxime as $98.0 \mathrm{k} . c a l$. is probably correct.

The quenching effects of benzaldcxime and oxygen on the phosphorescence of biacetyl were very similar and it has been well established that oxygen quenches the excited triplet states of biacetyl very efficiently (124). Under the experimental conditions employed in this investigation, it was sufficient to admit air into the evacuated cell in order to quench the phosphorescence of biacetyl.

Under aprotic conditions, use of aromatic and ketonic donors capable of transferring energy by triplet sensitization, led only to geometrical isomerization without any detectable amount of benzamide. The quantum yield ( $\left.\phi_{\mathrm{BZs}}\right)_{334}$ was less than .001 . By direct irradiation at $290 \mathrm{~m} \mu$ in cyclohexane and dioxane, $\left(\phi_{\mathrm{BZ}}\right)_{290}$ was 0.002 . These results suggest that only geometrical isomerization is involved and that reaction occurs from the triplet state of benzaldoxime. In the absence of benzamide, it must also mean that rearrangement does not occur from the same state. This, in turn, implies that upon direct absorption of light, those initially excited benzaldoximes in their singlet states utilize only $0.2 \%$ of the light to rearrange. The other $99.8 \%$ is either lost in a radiationless process or crosses over to the triplet state from which geometrical isomerization takes place. In fact, at least $78 \%$ of the light is transformed into the triplet 
state of benzaldoxime since the sum of the quantum yield of isomerism from $\alpha$ to $\beta\left(\phi_{\alpha \beta}\right)$ and from $\beta$ to $\alpha$ isomer $\left(\phi_{\beta \alpha}\right)$ is 0.78 in dioxane. Under aprotic conditions, however, the absence of benzamide was not entirely convincing that the amide could not be formed. Since the yield as well as the quantum yield were originally poor $\left(\phi_{\mathrm{BZ}}=0.002\right)$ upon direct irradiation at $290 \mathrm{~m} \mu$ a more suitable comparison is therefore, with the result obtained under conditions in which the yield and quantum yield were sufficiently high. Hence, when benzaldoxime, in the presence of acetic acid and triphenylene, was irradiated at $334 \mathrm{~m} \mu$, sensitized transformation of benzaldoxime to benzamide definitely occurred. The yield was very low. There is no doubt, however, that benzamide is formed. From the total amount of light absorbed by the sensitizer, the quantum yield $\left(\phi_{\mathrm{BZ}-\mathrm{S}}\right)_{334}$ of benzamide is 0.002 . Allowance for the intersystem crossing $\left(\phi_{\text {ISC }}=0.95\right)$ of triphenylene does not alter this value appreciably although it would account for the maximum triplet-triplet energy that can possibly occur upon sensitization. Compared with the quantum yield $\left(\phi_{\mathrm{BZ}}=0.025\right)$ obtained by direct irradiation under similar reaction conditions at $290 \mathrm{~m} \mu$, this sensitized efficiency was smaller by a factor of 13 . Assuming that triplet transfer was complete, the result would definitely indicate that benzamide formed by direct irradiation, was a consequence of excitation to the singlet state followed by rearrangement to the benzamide via an intermediate. There is validity in the assumption that the transfer is essentially complete from triphenylene to 
benzaldoxime based on the following consideration. The triplet-triplet energy transfer from biacetyl to benzaldoxime appears to indicate that transfer occurs by a highly diffusion controlled exorthermic process. The oxime is susceptible to sensitization by a donor of energy value as low as $43 \mathrm{k} . c a l$. Because the $\mathrm{E}_{\mathrm{T}}$ value of triphenylene is still greater than that of biacetyl by $10 \mathrm{k} . c a l .$, one would expect that the collisional energy transfer from triphenylene to benzaldoxime would be even more efficient than that from biacetyl to benzaldoxime and would expect a value very close to the maximum diffusion controlled rate. Recently, Kearne (140) reported that triplet transfer from acetone to 2-pentene is almost 100\% efficient despite the endothermicity of the reaction by $1000 \mathrm{~cm}^{-1}$ or $3 \mathrm{k} \cdot \mathrm{cal}$.

Assuming that triplet-triplet energy transfer occurs on every collision by a diffusion-controlled process (134) from triphenylene to benzaldoxime, the maximum second order rate constant in cyclohexane at room temperature is calculated to be $k_{D}^{A}=0.75 \times 10^{10} \mathrm{IMsec}^{-1}$, at $25^{\circ} \mathrm{c}$ using the equation $\mathrm{k}_{\mathrm{D}}=$ $8 R T / 3000$ (132). The concentration of benzaldoxime of $8.3 \mathrm{x}$ $10^{-3} \mathrm{MI}^{-1}$ used in the experiments is sufficient to quench the triplet of triphenylene at a pseudo first order rate constant of $\mathrm{k}=6.2 \times 10^{7} \mathrm{sec}^{-1}$. This rate is much faster than the rate of first order triplet decay of triphenylene, which is $1.8 \mathrm{x}$ $10^{4} \mathrm{sec}^{-1}$ in $\mathrm{n}$-hexane (115). 
Because of the low yield of benzamide by sensitization, the multiplicity of the state responsible for the photochemical Beckmann rearrangement can then be assigned to the singlet and that state responsible for the geonetrical isomerization assigned as the triplet. Upon sensitization, had the structural isomerization to benzamide occurred from the triplet, one would have expected to observe an exhancement in the quantum yield. A direct excitation to the triplet state would have been reached by by-passing the singlet-triplet intersystem crossing which can occur upon direct excitation of the oxime. The loss of energy from the singlet state reached by direct irradiation of the oxime would contribute to the decrease in the population of the triplet state by intersystem crossing.

Acetic acid in the solution containing the sensitizers does not quench the phosphorescence of benzophenone or triphenylene, therefore, quenching of the triplet state of triphenylene by acetic acid can be excluded as a reason for the low quantum yield of benzamide on sensitization. Even in the presence of acetic acid, triphenylene was found to be capable of effecting geometrical isomerization at a rate faster than the acid catalyzed conversion of the $\beta$ - to the $\alpha$-form. Although the $\alpha$-form was found to be highly favoured in acetic acidutrifluoroacetic acid, (100:I) sensitized reactions tend to increase the $\beta$-form. Because the results are consistent with the view that benzamide arises via an 
excited singlet state rather than through a triplet state, there must be a route by which the same singlet state, reached by direct irradiation, could also be populated through the initially formed triplet state.

One way in which a reversion from the triplet to the corresponding singlet level could be induced is by a thermal activation process represented by Eq. 6 .

$$
\text { Eq. } 6 \quad 3_{A} \stackrel{k_{e}}{\longrightarrow} I_{A} \longrightarrow \text { benzamide }
$$

The occurrence of such a process had been known as early as 1941 when Iewis first reported the observation of delayed fluorescence from fluorescein in boric acid glass (135). Parker and Hatchard $(136 a, b)$ have also demonstrated that the delayed fluorescence of eosin and proflavin, which is temperature dependent, is a result of the excited molecules in the triplet states being re-excited to the singlet state, from which fluorescence occurred. This type of thermal activated, delayed fluorescence has been termed E-Type delayed fluorescence and is essentially confined to systems in which the energy separation between the lowest excited singlet level and the triplet level is about $10 \mathrm{k} . \mathrm{cal}$. In a system in which both P-type and E-type delayed fluorescence are possible, and when the energy separation between the two corresponding excited singlet and triplet level of the order of 20-30 k.cal., then the thermal activation by an E-Type delayed fluorescence mechanism is considered to be negligible. 
The mechanism cannot be adequately described in terms of Eq. 6.

From the preceding discussion it is thought that the triplet energy of benzaldoxime is probably below 54-57 k.cal. and possibly as low as $43 \mathrm{k}$.cal. A triplet energy value of this magnitude for benzaldoxime would mean that the separation between the triplet energy level and the lowest excited singlet level could be as high as 4l-55 k.cal. In butadiene, $\Delta\left(E_{\mathbb{P}_{b-0}}-E_{S_{0-0}}\right)$ for $\left(\pi \pi^{*}\right)$ transition is as high as 60-75 k.cal. Even if the lowest triplet level of benzaldoxime were as high as that of styrene, which is 62 k.cal., a separation of energy between the two excited singlet and triplet levels of $36 \mathrm{k} . \mathrm{cal}$. would not be conducive to a thermal activation process represented by $\mathrm{Eq}$. 6 . The occurrence of such a mechanistic pathway would be highly improbable.

On the other hand, if two triplet molecules of benzaldoxime, $\left({ }^{3} \mathrm{~A}\right)$, are sufficiently long-lived, they may collide with one another in a solution and "annihilate" each other simultaneously, producing one molecule in an excited singlet state ${ }^{I_{A}}$ even of higher energy than $3_{A}$ and one in its ground state, $A$ of lower than ${ }^{3} \mathrm{~A}$ i.e.

Eq. $7 \quad{ }^{3} A+{ }^{3} A \longrightarrow{ }^{1} A+A$

In the benzaldoxime system this process could generate a 
singlet benzaldoxime molecule which in turn could eventually afford benzamide. The triplet-triplet annihilation reaction can proceed either with or without the formation of an excited dimer or an intermediate. Such reactions have been postulated by Parker and Hatchard to account for delayed fluorescence of aromatic compounds such as naphthalene, anthracene, pyrene, acenaphthalene and 3,4-benzpyrene. Delayed fluorescence defined in terms of $\mathrm{Eq} .7$ is usually referred to as P-Type delayed fluorescence (136c) and have been observed both by direct irradiation and by sensitization in solutions (136d,e,f). In the case of delayed fluorescence of naphthalene sensitized by phenanthrene, the system emitted light of higher frequency than that of the exciting source (I36d)so called anti stokes delayed fluorescence.

The triplet-triplet annihilation requires that part of the energy from two separately absorbed quanta be transferred to the same molecule by collision and in accordance with the conservation of energy, the mechanism demands that the triplet energy of ${ }^{3} \mathrm{~A}$ be at least one half the energy of the first excited singlet state, in order to generate ${ }^{I_{A}}$ by this two-photon annihilation process. Since the lowest singlet level of benzaldoxime is $98 \mathrm{k}$.cal., its triplet level should be at least $49 \mathrm{k} . \mathrm{cal}$. This value lies between $\mathrm{E}_{\mathrm{f}}$ values of biacetyl ( 54 - $57 \mathrm{k.cal}$. ) and anthracene ( $43 \mathrm{k.cal}$.$) . A$ triplet energy value $E_{T_{00}}=49 \mathrm{k}$.cal. would then mean an extremely large separation between the first excited singlet 
and the lowest triplet state. In contrast to benzaldehyde, this separation is only about $5 \mathrm{k.cal}$. ( $n \pi^{*}$ transition) whereas for styrene it is large and may be as much as $36 \mathrm{k}$. cal. ( $\pi \pi^{*}$ transition). A value of $E_{T}=49 \mathrm{k}$.cal. for benzaldoxime then would be sufficiently low to lead one to expect a diffusion controlled quenching rate between biacetyl and triphenylene with benzaldoxime. Parker and Joyce (136f) have successfully estimated the triplet energy of perylene from their studies of sensitized delayed fluorescence of anthracene. Since sensitization of perylene by anthracene is extremely efficient ( $\left.E_{-p}=43 \mathrm{k} \cdot \mathrm{cal}_{\bullet}\right)$ and delayed fluorescence of perylene also occurs, the limit of $\mathrm{F}_{\mathrm{T}}$ for perylene was thought to be between $43 \mathrm{k}$.cal. and one half the singlet value of perylene $\left(\frac{1}{2} \mathrm{E}_{\mathrm{So}-\mathrm{O}}=33 \mathrm{k}\right.$.cal.). The observed value is $36 \mathrm{k} . c a l$. If an analogous argument were extended to the triphenylenebenzaldoxime system, the $E_{T}$ value of benzaldoxime would then lie between 67 and $49 \mathrm{k.cal}$.

In accordance with the triplet-triplet annihilation mechanism by which the delayed production of benzamide from singlet monomer is assumed, and assuming that the following simplified scheme represents the processes of delayed production of singlet,
Eq. 8
$D \stackrel{h \nu}{\longrightarrow} I_{D}$
$=I_{a}^{\text {rate }}$
Eq. 9
$I_{D} \rightarrow 3_{D}$
$=\phi_{\text {isc }}^{D} \cdot I_{a}$
Eq. 10
${ }^{3} D+A \longrightarrow D+{ }^{3} A$
$=k q \cdot{ }^{D} D \cdot A$ 


\begin{tabular}{|c|c|c|}
\hline $\mathrm{Eq} \cdot 11$ & ${ }^{3 D} \rightarrow D$ & $=k_{h} \cdot{ }^{3} 0$ \\
\hline Eq. 12 & ${ }^{3} \mathrm{~A} \rightarrow \mathrm{A}$ & $=k_{j} \cdot{ }^{3} A$ \\
\hline $\mathrm{Eq} \cdot 13$ & ${ }^{3} \mathrm{~A}+{ }^{3} \mathrm{~A} \longrightarrow{ }^{I_{A}}+\mathrm{A}$ & $=A_{g} \cdot(3,4)^{2}$ \\
\hline Eq. 14 & $I_{A} \leftrightarrows X$ & $=z_{i} \cdot A$ \\
\hline Eq. 15 & $\mathrm{X} \longrightarrow$ Benzamide ( $\left.B_{z}\right)$ & $=k_{p} \cdot x$ \\
\hline
\end{tabular}

the order of magnitude of the triplet life time can be estimated. In this scheme, $I_{a}$ is the rate of absorption of light, $\phi_{\text {ISC }}^{D}$ is the quantum yield of triplet formation of the donor, $k_{h}$ and $k_{j}$ are composite first order rate constants for the disappearance of the triplet. These include pseudo first order contributions due to possible quenching by impurities in the solution. $\mathrm{kg}$ is a second order rate constant for the triplet energy transfer from donor to benzaldoxime and can be assumed to be of the order of the diffusion controlled rate since the $E_{T}$ value of the benzaldoxime appears to be below that of triphenylene. $p \cdot$ is the self-quenching triplet rate. " $p$ is the probability that every encounter between triplets, ${ }^{3} \mathrm{~A}$, will give rise to an excited singlet, ${ }^{I_{A}}$.

The quantum yield of delayed conversion of benzamide $\left(\theta_{\mathrm{BZ}}\right)$ can be estimated utilizing equations 16,17 , and 18 . Eq. 16

$$
\theta_{B_{z}}=\frac{\phi_{B_{x}} \cdot \frac{d^{\prime} A}{d t}}{I_{a}}
$$

Eq. 17

$$
B_{D}=\frac{\phi_{1 S C}^{D} \cdot I_{a}}{\left(\phi_{g} \cdot A+k_{h}\right)} \quad \text { or } \quad B_{A}=\frac{k_{q} \cdot A \cdot\left|B_{D}\right|}{k_{j}}
$$

Eq. 18

$$
\theta_{B z}=\frac{1}{2} \cdot p \cdot A_{q} \cdot I_{a} \cdot\left(\phi_{1 S C}^{D} \cdot \tau_{3_{A}}\right)^{2} \text { or }\left(\tau_{B_{A}}\right)^{2}=\frac{2 \theta_{B z}}{\left(\phi_{1 S C}^{D}\right)^{2} \cdot p k_{q} \cdot I_{a}}
$$


A number of assumptions has been made and they are the following: a) a steady state concentration of ${ }^{3} \mathrm{D}$ and ${ }^{3} \mathrm{~A}$ occurs, b) the rates of triplet-triplet interaction by process 13 is smaller than the rates of radiationless conversions, c) the life time of the triphenylene triplet is essentially defined by process 10*, and d) that step 14 is an irreversible process where $X$ is an intermediate which affords benzamide ( $\mathrm{Bz})$.

$\phi_{B z}$ is defined as the quantum yield obtained by direct irradiation and $T_{\beta_{A}}$ is the life time of benzaldoxime triplet in cyclohexane. Inserting $\phi_{\mathrm{BZ}}=0.025, \theta_{\mathrm{BZ}}=0.002$,

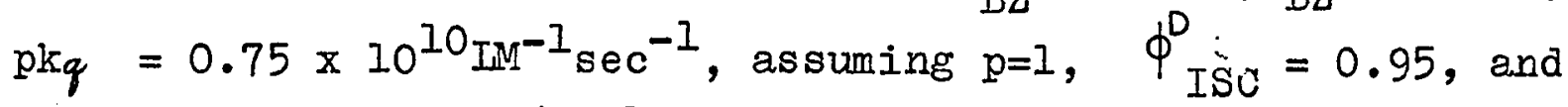
$I_{a}=2 \times 10^{-5}$ eins. ${ }^{-I_{[s e c}^{-1}}$, then $\tau_{3 \mathrm{~A}}$ is calculated to be $=4$ $x 10^{-3} \mathrm{sec}$ or $4 \mathrm{~m} \mathrm{sec}$.

Upon direct irradiation of benzaldoxime, the quantum yield for delayed formation of benzamide $\left(\theta_{B z}\right)$ can be analogously expressed as:

Eq. $19 \quad \theta_{B z}=\phi_{B_{2}} \cdot \rho k_{q} \cdot I_{2} \cdot\left(\phi_{1 s c}^{A} \cdot \tau_{B_{A}}\right)^{2}$

where $\phi_{i s c}^{A}$ is the quantum yield for the intersystem crossing of benzaldoxime. Then, an estimate of $\phi_{\text {ISsc }}^{A}$ can be made since geometrical isomerization occurs largely through a

*In $n$-hexane, the first order rate constant for the decay of triphenylene triplet is $18 \times 10^{3} \mathrm{sec}^{-1}(125)$. Assuming that the quenching of the triplet by benzaldoxime is diffusion controlled $\left(k_{\mathrm{d}}=0.75 \times 10^{10} \mathrm{IN}^{-1} \mathrm{sec}^{-1}\right.$, then in the presence of $8.3 \times 10^{-3} \mathrm{MI}^{-I}$ of the oxime, the first order rate of quenching will be $6 \times 10^{-1} \mathrm{sec}^{-1}$. This value is greater by a factor of 3500 than the first order rate constant wr the trinlet deray 
triplet state. The sum of the quantum yields for geometrical isomerization was determined as 0.78 . Using this value, as

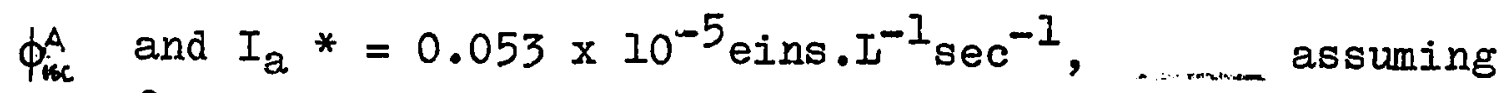
that $\theta_{\mathrm{Bz}}=0.002$, then $\tau_{\mathrm{A}}=6 \times 10^{-3} \mathrm{sec}$. The only difference between equations (18) and (19) was the choice of $\left|\phi_{i s c}^{A}\right|,\left|\phi_{s c}^{D}\right|$, and $I a$. Therefore the lifetime $\left(T_{B}\right)$ of the benzaldoxime triplet is estimated to be approximately $10^{-3}$ sec. For those compounds which have been observed to undergo P-type fluorescence, the lifetimes are found to be near $10^{-3} \mathrm{sec}$. For example, the lifetime of perylene determined by Parker and Joyce $(136 \mathrm{~g})$ is $3.92 \times 10^{-3} \mathrm{sec}$. and for aromatic hydrocarbons such as acenaphthalene, naphthalene, benzanthrene, and pyrene, the lifetimes of the triplets in ethanol vary from $3.3 \times 10^{-3} \mathrm{sec}$. to $10.8 \times 10^{-3} \mathrm{sec}$.

Under the conditions of sensitization there is still another mode of tiriplet-triplet annihilation which can possibly account for the excitation of the benzaldoxime triplet to the first excited state. This involves a mixed triplet quenching between the donor triplet and triplet of benzaldoxime as represented by equation (20);

$$
\text { Eq. } 20 \quad{ }^{3} D+{ }^{3} \mathrm{~A} \longrightarrow \mathrm{D}+{ }^{1_{A}} \quad \text { rate }=k g, 3^{3} \cdot 3_{A}
$$

In general, it is expected that both processes, (13) and (20) can occur simultaneously but, with excess acceptor, excitation by (13) rather than (20) should predominate, especially if triplet transfer from the donor to the

*Intensity value obtained by direct irradiation at $290 \mathrm{m \mu}$. 
acceptor is highly efficient. If the population of the delayed singlet upon sensitization can be represented by process 20 rather than by $13, \tau_{3 \mathrm{~A}}$ can be estimated to be $4 \times 10^{2} \mathrm{sec}$. (using equation 21).

$$
\begin{aligned}
& \text { Eq. } 21 \quad \dot{\theta}_{B_{2}}=\phi B_{3} \cdot k_{q}{ }^{2}[A]\left[T_{B_{A}}\right]\left[\phi_{I S C} \cdot T_{B_{D}}\right]^{2} \text { and } \\
& \tau_{3_{D}} \approx \frac{1}{k_{h}+k_{q^{A}}+k_{d^{3} A}} \approx \frac{1}{k_{q_{A}}} \approx 16 \times 10^{-7} \mathrm{sec}
\end{aligned}
$$

since the lifetime of the triphenylene triplet will be essentially defined by $\mathrm{k}_{\mathrm{q}} \mathrm{A}$. If the lifetime of the benzaldoxime triplet is as long as $400 \mathrm{sec}$, one might expect to observe phosphorescence. Instead, no emission of any kind was observed. Therefore, process (13) is favoured as the probable mode of triplet-triplet quenching responsible for the production of singlet benzaldoxime ${ }^{l_{A}}$.

The relative rates of other photophysical processes, such as radiationless transitions or quenching effects could account for the lack of emission.* Since the sum of the quantum yields of geometrical isomerization of 0.78 and the maximum yield of benzamide is 0.058 , only about $16 \%$ of the initially absorbed enelify must be dissipated by radiationless transitions or quenching. There are instances reported in which phosphorescence represents a very small fraction of utilization of light, which underwent intersystem crossing from the singlet to the iriplet state $(136 \mathrm{~h})$. Although the quantum yield of

* The lifetime of the benzaldoxime triplet estimated in the manner described is comparable to that observed for many aromatic compounds, and most of the latter compounds emit light as phosphorescence or fluorescence. Compounds with an even shorter Iifetime $\left(10^{-3} 3 \mathrm{sec}\right)$ are known to enit $136 \mathrm{~g}$, ref. $14, \mathrm{p} .297$. 
naphthalene in ethanol solution is $\phi_{\text {ISC }}=0.65,(136 \mathrm{~h})$ the quantum yield of phosphorescence $\left(\phi_{p}\right)$ in a rigid medium is only $\phi_{p}=0.008$ to $.02(136 i, 137)$ which represents $2-3 \%$ of the amount that crosses over from singlet to triplet states, with 95-98\% being dissipated by radiationless transitions or being utilized in the chemical reaction. Kearne (138)** has reported that in glycerol, at $77^{\circ} \mathrm{K}$, only $1 \%$ of acetone phosphoresces. The loss of energy from the acetone iriplet would be due to reaction with the solvent as a primary process, to the extent of about $\phi=.40$ in cyclohexane.

\section{Cis-Trans Isomerization}

A brief discussion of the photoisomerization process for benzaldoxime is included since some of the results have a bearing on the structural isomerism itself.

Firstly, the geometrical isomerization is more efficient than the structural isomerism by a factor of about 13 and this means that most of the radiant energy initially absorbed by benzaldoxime is utilized for geometrical isomerization. The sum of the quantum efficiencies $\left(\phi_{\alpha \beta}+\phi_{\beta \alpha}\right)$ in both directions in dioxane was 0.78 at $290 \mathrm{~m} \mu$, leaving about $22 \%$ of the radiant energy for other processes. Since rearrangement is negligible in dioxane $\left(\phi_{\mathrm{BZ}}=0.002\right)$ and there is no emission most of the excess energy must have been lost, by radiationless decay to the surroundings or by solvent quenching.

Upon direct irradiation and photosensitization with triplet donors, the photostationary state is quickly reached.

$$
\text { ** } \text { - ref. } 139 \phi_{\text {ISC }}=0.40
$$


When benzaldoximes are exposed to unfiltered light from a high pressure mercury lamp and to a monochromatic beam at 253.7m $\mu$ from a low pressure mercury lamp, the ratios of $\beta$ benzaldoxime to $\alpha$-benzaldoxime are 2.0 and 1.3 , respectively, with a preponderance of the thermodynamically less stable B-isomer.

Because of the similar quantum efficiencies of geometric al isomerization in both directions, the observed photostationary state merely reflects the difference in the extinction coefficients or the relative rates of excitation of the two isomers. Furthermore, at the wavelengths employed, photostationary state conditions are reached in which the $\beta$-form predominates because the $\alpha$-form is excited at the faster rate. That such a process is responsible for the observed effect can be shown if one assumed that Beer's Law is obeyed under all conditions of the experiment and that the quantum yield in both is independent of intensity $\left(I_{0}\right)$. Then according to Zimmerman (152) the differential equation (22) for the rate of formation of $\beta$-benzaldoxime excited by $a$ monochromatic beam can be written as:

$E q .22 \frac{d B}{d t} * \frac{I_{0}}{V F}\left(\phi_{\alpha \beta} \cdot \epsilon_{\alpha} \cdot[\alpha]\right)-\frac{b}{V F}\left(\phi_{\beta \alpha} \cdot \epsilon_{\beta} \cdot[\beta]\right)-k[\beta]$

The following symbols are defined as:

$I$ = path length of the cell

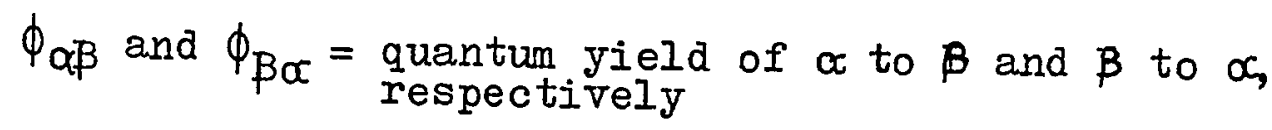




$$
\begin{aligned}
& E=\text { molar extinction coefficients } \\
& {[\alpha] \text { and }[\beta] \text { are expressed in mole fractions }} \\
& D=\text { optical density } \\
& V=\text { volume of solution in Iitre } \\
& F=\frac{D}{1-e^{-D}} \\
& t=\text { time }(\mathrm{sec}) \\
& I_{0}=\text { einstein/sec. }
\end{aligned}
$$

The last term, which accounts for thermal isomerization $\beta \rightarrow \alpha$, can be neglected since thermal isomerization is much slower than photochemical process. Both $\alpha$-and $\beta$-isomers are stable in non-protonic solvents. At the photostationary state then, the rate of formation $\left(\frac{\partial \beta}{d t}\right)$ is zero. Hence,

$\mathrm{Eq} \cdot 23$

where $\left(\frac{\beta_{\infty}}{\alpha_{\infty}}\right) \lambda_{i}$ represents the relative amounts of $\beta$-and $\alpha$ isomers under stationary state conditions. Assuming that the relative quantum yield of isomerization $\left(\frac{\phi_{\alpha \theta}}{\phi_{\alpha \alpha}}\right)_{\lambda_{i}}$ is independent to wavelength, i.e.

$E q \cdot 24 \quad\left(\frac{\phi_{\alpha \beta}}{\phi_{\beta \alpha}}\right)_{253.7}=\left(\frac{\phi_{\alpha \beta}}{\phi_{\alpha \alpha}}\right)_{290}$

then the predicted value of $\left(\frac{\beta_{\infty}}{\alpha_{\infty}}\right)_{23.7}$ at $253.7 \mathrm{~m} \mu$ is calculated as 1.3, in good agreement with the experimental value, 1.3.

As a further test for the validity of the assumption that $\left(\frac{\phi_{\alpha \theta}}{\phi_{\beta a}}\right)_{\lambda_{i}}$ is ind ependent of wavelength, the predicted 
value $\left(\frac{\beta_{\infty}}{\alpha_{\infty}}\right)$ due to irradiation with unfiltered light from a mercury lamp is calculated by applying the Eq. 23. The calculated ratio, $\left(\frac{\beta_{\infty}}{\alpha_{\infty}}\right)_{\lambda_{i}}$ was 2.0 , in good agreement with the experimental value of 2.0. For the last calculation, the effective pelative rate of excitation $\left(\frac{\epsilon_{\alpha}}{\epsilon_{\beta}}\right)$ was determined by considering the three main lines at 289, 297, and $302 \mathrm{~m} \mu$ from the high pressure mercury lamp, absorbed by benzaldoxime. Since the relative molar extinction coefficients $\left(\frac{\epsilon_{\alpha}}{\epsilon_{\beta}}\right)_{\lambda_{i}}$ obtainable from the absorption spectra are $1.2,2.9,1.3$ in dioxane and the respective relative intensities of the emission lines from the lamp were $4 \%$, $9 \%$, and $13 \%$, effective relative rate of absorption is 1.9 .

Although the ratio $\left(\frac{\phi_{\alpha \theta}}{\phi_{\text {ex }}}\right)_{290}$ determined at $290 \mathrm{~m} \mu$ appears to be constant, it does not prove that the individual quantum efficiency of geometrical isomerization is the same at all wavelengths. Since excited singlets have extremely short lifetimes $\left(10^{-9}\right.$ sec. $)$ and internal conversion from upper excited states above the 0-0 level of the first excited state $\left(E_{S_{-}}\right)$to the latter level is extremely fast, it seems probable that $\phi_{\alpha \beta}$ and $\phi_{\alpha \alpha}$ are the same at all the wavelengths above $253.7 \mathrm{~m} \mu$ for which data are available.. If this last condition is indeed the actual representation of the photophysical process, then the dependence of the rearrangement of benzaldoxime to benzamide upon the energy of excitation cannot be attributed to eppreciable singlettriplet intersystem crossing at ?evels above the lowest 
vibrational level of the first excited state. This has been suggested (see section under the Effect of Wavelength) as one of the possible causes of reduction of the rearrangement yield at $253.7 \mathrm{~m} \mu$. The lack of dependence of the geometrical isomerization on the wavelength of the exciting source would also mean that intersystem crossing from the singlet level to the triplet level probably occurs from the 0-0 level of the first excited state. In dioxane, $E_{\text {So-o }}$ levels for $\alpha$-benzaldoxime and $\beta$-benzaldoxime are $292 m \mu(98.2 \mathrm{k.cal}$.$) and$ $290 \mathrm{~m} \mu(98.8 \mathrm{k} . \mathrm{cal}$.$) respectively, showing a separation for$ the singlet levels of the two benzaldoxime isomers to be $0.6 \mathrm{k} . \mathrm{cal}$. These are comparable to the separation of ground state levels $(0.66 \mathrm{k} . \mathrm{cal}$.) reported by Swietoslawski (I53).

The photostationary state rates $\left(\frac{\beta_{\infty}}{\alpha_{\infty}}\right)$ Sensiotained by the use of triplet donors with energy above $62 \mathrm{k} . \mathrm{cal}$. are within the range $1.6-1.8$, indicating that the transfer of energy $(160,182)$ from donor to benzaldoxime is essentially independent of the triplet energy of the former. This behaviour is very similar to that of piperylene, stilbenes, and azobenzene $(128,183)$. Invariably, where the transfer of triplet energy from donors to acceptor molecules capable of undergoing geometrical isomerization is highly exothermic, the photostationary state conditions have been found to be independent of the triplet energy value. The phenomenon has been regarded by Hammond as the result of a highly diffusion con- 
trolled energy transfer from the donor to the acceptor. There is much evidence to support such a claim. Studies of quenching efficiencies have been found to fall off when the energy of the excited donors is just above the excitation of the acceptors (117c). For benzaldoxime, analogous energy transfer processes occur and, therefore, the triplet energy $E_{\mathbb{T}}$ must be below $62 \mathrm{k}$.cal., which represents the value of the lowest triplet energy donor used in attaining a photostationary state by sensitization. On the basis of the previous discussion which invoked the participation of the triplettriplet annihilation mechanism, a safe and reasonable estimate of the $E_{T}$ level of benzaldoxime is between 49 and $62 \mathrm{k.cal}$. and possibly as low as 49-57 k.cal., as suggested by the efficient quenching of phosphorescence emission of biacetyl.

From the absorption spectra of $\alpha$ - and $\beta$-benzaldoxime and the heat of isomerization of $\beta$ to $\alpha$-benzaldoxime reported by Swietoslawski (153), the energy separation between the two isomers is of about the same order of magnitude in both the excited singlet and the ground states. The appearance of the absorption spectra strongly suggests that both forms do not differ energetically to any large extent because of steric effects, since the relative intensity of absorption appears to be about the same. The spectra differ only in the position of the maxima. For the benzaldoxime isomers(in cyclohexane and in n-propanol cyclohexane solvent system $(1: 4)$, the extinction coefficients of the 
highest absorption band in the region of 240-260m $\mu$ are identical, unlike molecules such as cis- and trans-stilbene which show a very large difference in intensity of absorption (154). It seems likely that both spectroscopic triplet states of these two $\alpha$-and $\beta$-isomers of benzaldoxime differ to about the same extent, i.e. $1 \mathrm{k} . \mathrm{cal}$. The barrier to interconversion between these states is probably very small. At high temperatures, a very facile conversion between two spectroscopic triplet states probably occurs due to the small energy barrier. This would then lead to a rapid equilibrium. That such a situation may exist for the oximes is founded on the following grounds. Malkin and Fisher (155) have recently reported that isomerizations of compourds with symmetrical central double bonds $(\mathrm{C}=\mathrm{C}$ and $\mathrm{N}=\mathrm{N}$ ) are temperature dependent. In contrast, the isomerization of compounds with unsymmetrical central double bond $(\mathrm{CH}=\mathbb{N})$ is temperature independent.

A relationship describing the kinetics of the geometrical isomerization (Fig. 12) and consistent with the present results can be derived following the method proposed by Stegmeyer (156). The derivation of the equations 25-27, relating the quantum yields to the rate constants, allows for the possible - interconversion between two spectroscopic triplet states of $\alpha$ - and $\beta$-benzaldoximes. 
Eq. $25 \quad\left(\phi_{\alpha \beta}\right)_{\lambda \bar{i}}\left(\frac{k_{2}}{k_{2}+k_{5}}\right) \cdot\left(\frac{k_{3} \cdot k_{4}}{k_{3} \cdot k_{4}+k_{3}^{1} k_{4}^{1}+k_{4} k_{4}^{1}}\right)$

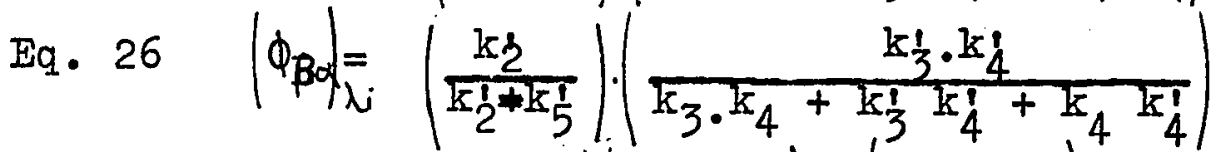

Eq. $27 \quad\left(\frac{\phi_{\alpha \beta}}{\phi_{\beta \alpha}}\right)=\left(\frac{k_{2}}{k_{2}+k_{5}}\right)\left(\frac{k_{2}+k_{5}^{1}}{k_{2}}\right) \cdot\left(\frac{k_{3} k_{4}}{k_{3}^{\prime}+k_{4}^{\prime}}\right)$

For the sensitized photoisomerization represented by the processes 28-32:

Eq. $28 \quad \alpha+s^{*} \stackrel{k_{t}}{\longrightarrow} 3_{\alpha}+S$

Eq. $29 \beta+\mathrm{s}^{*} \stackrel{\mathrm{ke}}{\longrightarrow} 3 \beta+\mathrm{s}$

Eq. $30 \quad 3 \propto \underset{\mathbf{k}_{i}^{\prime}}{\mathrm{m}} \propto$

Eq. $31 \quad 3 \beta \stackrel{k_{4}}{\longrightarrow} \beta$

Eq. $32 \quad 3_{\alpha} \quad \frac{3}{\frac{3}{3}} 3^{3}$

then the sensitized photostationary state is given by equation 33 and 34 , assuming $k_{t}=k_{c}$ due to comparable diffusion controlled energy transfer rates from the sensitizers to benzaldoximes.

Eq. $33\left(\frac{\beta_{\infty}}{\alpha_{\infty}}\right)_{\text {sens. }}=\frac{k_{t}}{k_{c}} \cdot \frac{k_{3}}{k_{\frac{1}{3}}^{\prime}} \cdot \frac{k_{4}}{k_{4}}=\frac{k_{3}}{k_{\frac{1}{3}}} \cdot \frac{k_{4}}{k_{4}} \quad$ or

Eq. $34 \quad \frac{k_{3} \cdot k_{4}}{k_{3}^{1} \cdot k_{4}^{!}}=\left(\frac{B_{\infty}}{\alpha_{\infty}}\right)_{\text {sens. }}=1.7$

Then combining the equations 25,26 and 33 gives the following relationships :

Eq. 35

$$
\left(\frac{\phi_{\alpha g}}{\phi_{\beta \alpha} \lambda_{i}} \cdot \frac{1}{1 \cdot 7}=\frac{k_{2}}{k_{2}+k_{5}} \cdot\left(\frac{k_{2}^{1}+k_{5}^{1}}{k_{2}^{1}}\right)=0.65\right.
$$


A radiationless dissipation of energy from the excited singlet is demanded by this mechanism since $\frac{\phi_{\alpha \beta}}{\phi \beta \alpha} \frac{1}{1.7}$ should be 1 . if $k_{5}$ and $k_{5}$ are negligible. Therefore, the radiationless decay of the singlet states of the two oximes is suggested to account for the "missing" $22 \%$ of the energy when the oximes are excited directly. The low yield of benzamide can then be attributed to the efficient depopulation of the singlet state merely by radiationless decay from the excited state or by solvent quenching, which in this case, $\mathrm{k}_{5}$; will represent a pseudo first order rate constant, in addition to the facile intersystem crossing that occurs from the excited singlet level to the triplet state in benzaldoxime.

Under conditions of sensitization by exothermic triplet energy transfer from the donors to the oximes, and conceiving the possibility of the temperature independence of the geometrical isomerization, the ${ }_{3}$ and $\mathbf{k}_{3}$.. would probably be of the same order of magnitude (156). At the stationary state, the relative amounts of $\beta$-and $\alpha$-isomer will then be represented by the ratio:-

$$
\mathrm{Eq} \cdot 36 \quad\left|\frac{\mathrm{B}_{\infty}}{\alpha_{-}}\right|_{\therefore}=\frac{\mathrm{k}_{4}}{\mathrm{k}_{4}^{\prime}}
$$

where the observed photostationary state will merely reflect the relative rate of decay from the triplet states of $\beta$-and $\alpha$-benzaldoxime. At the outset, Hammond's simple formulation (124) predicting the photostationary state upon direct irradiation under similar circumstances would also appear to 
apply to the benzaldoxime system if the photostationary state condition is independent of the triplet energy. This can be written as:

$$
\text { Eq. } 37\left(\frac{\beta \infty}{\alpha_{\infty}}\right)_{\lambda_{i}}=\left(\frac{\beta_{\infty}}{\alpha_{\infty}}\right)_{\text {sens. }} \cdot\left(\frac{\varepsilon_{\alpha}}{\varepsilon_{\beta}}\right)_{\lambda_{i}}=\left(\frac{\phi_{\infty}}{\phi_{\beta}}\right)_{\lambda_{i}} \cdot\left(\frac{\varepsilon_{\alpha}}{\sigma_{\beta}}\right)_{\lambda_{i}}
$$

The last equality must also be true since the observed photostationary state rates $\left(\frac{\beta_{\infty}}{\alpha_{\infty}}\right) \lambda_{i}$ upon direct irradiation depend only on $\left(\frac{\epsilon_{\alpha}}{\epsilon_{\beta}}\right)_{i}$.

For such a relationship to be applicable, $\left(\frac{\beta_{\infty}}{\alpha_{\infty}}\right)_{\text {sens. }}$. must be equal to $\left(\frac{\phi_{\alpha \beta}}{\phi_{\beta \alpha}}\right)_{\lambda_{i}}$ and this equality was certainly not obtained experimentally. The former ratio was greater than the latter by a factor of 1.6. Generally, the simple relationship represented by equation (37) cannot be applicable for benzaldoxime because the observed ratio, upon direct excitation, will always depend upon the relative rate of excitation at a particular wavelength, i.e. upon $\left(\frac{\epsilon_{\alpha}}{\epsilon_{\beta}}\right)_{i}$. Moreover, $\left(\frac{\beta_{\infty}}{\alpha_{\infty}}\right)$ sens. Should not be expected to be equal to $\left(\frac{\phi_{\alpha \beta}}{\phi_{\beta \alpha}}\right)_{i}$ since the former value reflests the decay of the excited triplet upon direct excitation to the triplet by sensitization. $\left(\frac{\phi_{\alpha \beta}}{\phi_{\beta \alpha}}\right) \lambda_{i}$, on the other hand, will always include terms a of crossing terms, ( $\phi_{\text {ISC }}$ ). 
Eq. $38 \phi_{\alpha \beta}^{\beta} \phi_{\beta \alpha}=\frac{\phi_{I S C_{\alpha}}}{\phi_{I S C_{\beta}}} \cdot \frac{k_{4}}{k_{4}}$ where $\frac{k_{2}}{k_{2}+k_{s}}=\left(\phi_{I S C}\right)_{\alpha}$ and $\frac{k_{2}^{\prime}}{k_{2}^{\prime}+k_{j}^{\prime}}=\left(\phi_{\text {ISC }}\right)_{\beta}$

Based on the brief study of isomerization using only a few sensitizers, a detailed description of the isomerization can not be made and does not warrant the postulation of twisted triplets or excited triplet states in perpendicular geometrical configurations. At present, its contribution to the phutochemical processes appears to be insignificant, since it would be energetically impossible to regenerate a first excited singlet state of benzaldoxime by a triplettriplet annihilation process. The mechanism will demand imparting energy to one single excited singlet molecule of at least $98 \mathrm{k}$.cal." of energy from one excited triplet to another. A triplet in its perpendicular configuration could have an energy as low as 73 kcal and this would demand 7-8 collisions between excited "phantom" triplets (twisted triplets) to excite one molecule of benzaldoxime possessing energy of $98 \mathrm{k} . \mathrm{cal}$. Even a mixed triplet-triplet annihilation process between the triphenylene would also fall short of $98 \mathrm{k}$.cal.

The energy of the "phantom"triplet (twisted triplet), if such a state can be reached by non-vertical excitation, can be estimated if one can assume that the magnitude of the activation energy required for thermal isomerization is a reflection of the most stable configuration of an excited 
state in its twisted geometrical configuration. From the data reported by Patterson (156) for the thermal conversion of $\beta$. to $\alpha$-isomer, the activation energy $\left(E_{a}\right)$ was calculated as $13 \mathrm{k.cal}$. The frequency factor was $7 \times 10^{6} \mathrm{sec}^{-I_{*}}$. The activation energy is in good agreement with the activation energy of $14-18 \mathrm{k} \cdot \mathrm{cal} . \mathrm{M}^{-1}$ required for thermal conversion of N-benzylidene-aniline about the $\mathrm{C}=\mathrm{N}$ band (158-159).

The preponderance of thermodynamically unstable $\beta$-isomer, upon direct irradiation and photosensitization, strongly suggests that the production of the ground state isomer aftet excitation is a consequence of a direct degradation of the electronically excited triplet states. In view of the possible small energy separation between the excited states, whether these be singlets or triplets at room temperature, the interconversion in both directions could be so much faster than degradation processes, i.e. $k_{3} \gg k_{4}$ and $k_{3}^{\prime}{ }^{\prime} \gg k_{4}^{\prime}$ (156). A rapid equilibrium might be established between these excited states with the preponderance of the $\alpha$-triplet. Under sensitization, if this were true, one might expect to find a greater proportion of $\alpha$ - than $\beta$-isomer as thermally attained upon equilibration. The results however, clearly indicate a preponderance of the $\beta$-form. Therefore, it might very well mean that this is a consequence of a direct degradation of the excited states.

* ref. (160) On the basis of kinetic studies of geometric isomerization about an ethylene bond, the reaction has been divided into two classes, one in which the activation energy is $20-25 \mathrm{k}$.cal. $\mathrm{M}^{-1}$ with frequency factor of 104-105, and the other in which the activation energy is $(40-45)$ and frequency factor is ajout 10 II. 
Presently, the experimental data are inadequate to exclude completely the occurrence of geometrical isomerization via the excited singlet states in the case of direct excitation of benzaldoximes.

\section{Quenching by Oxygen}

The results clearly show that the presence of oxygen in dioxane during the irradiation of benzaldoxime is ineffective in suppressing the quantum efficiency of geometrical isomerization $\left(\phi_{\alpha \beta}\right)$, whereas in cyclohexane, in the presence of acetic acid and oxygen, the quantum yield of benzamide $\left(\phi_{B z}\right)_{290}$ is reduced by a factor of three (Table IV). In the previous sections $V$ and $V I$, the intervention of the singlet and triplet states has been proposed for structural and geometrical isomerization respectively. In view of this, one is forced to conclude that oxygen is ineffective in quenching the triplet of benzaldoxime*. The lack of triplet quenching by oxygen is striking since oxygen is known to be an effective quencher of triplet states for many compounds. Both benzaldoxime and oxygen were demonstrated to quench the phosphorescence of biacetyl and, in the case of oxygen, Backstrom and Sandros (16I) have determined the quenching constant of biacetyl triplet by oxygen, in hexane at room temerature, to be $0.5 \times 10^{10} \mathrm{IM}^{-1} \mathrm{sec}^{-1}$, a value vary close to the rate constant defined by the maximum diffusion controlled

*Zimmerman (152) reports that oxygen in solution (2 $\times 10^{-7}$ to $1 \times 10^{-2} \mathrm{M}$ ) has no observable effect on the geometrical isomerization of diazobenzene. Kearne (164) reports that isomerization via triplet state is theoretically possible. Hammond (165) suggests both singlet and triplet states intervene. 
rate. Even triplets of aromatic compounds, such as anthracene, are quenched at rate constants $\left(0.2 \times 10^{10} \mathrm{IM}^{-1} \mathrm{sec}^{-1}\right)$ comparable to diffusion controlled rates in bromobenzene (162). It is even more remarkable that the reduction in $\phi_{\alpha \beta}$ was not observed when irradiated in the presenceroygen since bubbling of the gas through dioxane can lead to the formation of peroxides which in turn may be capable of interfering with the reaction (ref. 163, p.175).

The lack of quenching of geometric isomerism cannot be attributed to a rapid consumption of oxygen at the initial stage of irradiation. Under the experimental conditions of the reaction, assuming that the solubility of oxygen in dioxane is comparable to that in ethyl ther at room temperature (166), the volume of the irradiated solution is $300 \mu \mathrm{I}$ would contain $0.6 \times 10^{-5}$ Moles. of oxygen in solution. In one instance, the amount of light absorbed by benzaldoxime ( $1.0 \times 10^{-5}$ Moles) was $1.0 \times 10^{-5}$ einstein and, therefore, the amount of oxygen present would have been sufficient to quench two thirds of the excited states of benzaldoxime. Further, had the intersystem crossing from the excited singlet to the triplet been about 0.78 , the amount of triplet capable of being quenched by oxygen would have been as high as $80 \%$. The lack of quenching of the benzaldoxime triplet must be attributed to factors peculiar to the molecule itself.

On the other hand, in cyclohexane, oxygen was continuously supplied to the solution throughout the irradiation. 
In cyclohexane, oxygen dissolves to the extent of $2.5 \mathrm{x}$ $10^{-3} \mathrm{M}^{\prime}:$ or $\left(0.013 \times 10^{-3}\right.$ Moles. in $\left.5 . \mathrm{ml}\right)$ at room temperature, and, since the period of irradiation was 20 hours, a constant supply of oxygen over this period amounted to about $0.26 \times 10^{-3}$ Moles. This quantity of oxygen would have been capable of reacting with every excited molecule of benzaldoxime since the quanta absorbed by the solution, in one instance, is $0.26 \times 10^{-3}$ eins.

The fact that structural isomerization to benzamide is quenched by oxygen rather than geometrical isomerization must imply that oxygen interferes with one of the step(s) between the primary process and the final step leading to benzamide. The oxygen effect, however, may not be restricted entirely to a quenching of an electronically excited state because only the over-all quantum yield of benzamide was measured. The rearrangement of benzaldoximes to benzamides appears to involve the intermediacy of an oxazirane. Therefore, it is possible that oxygen could interrupt the final step by reaction with a radical-type intermediate. Alicyclic oxaziranes which are not substituted on nitrogen are thought to decompose by a free-radical mechanism (110b). Thus for benzaldoxime, the quenching effect of oxygen on the over-all yield of benzamide can be interpreted in one of two ways: either oxygen interferes with the primary process* by quenching the excited singlet of benzaldoxime, or it prevents the final conversion of the intermediate oxazirane to

* ref. 856 Keneko reports that oxygen interfered with the formation of oxazirane of quinolene-N-oxides. 
benzamide. Tanaka $(68,69)$ has assigned the singlet state from which the primary product, oxazirane, is formed from the corresponding nitrone, but found that oxygen was ineffective in quenching this transformation. Because the nitrone and benzaldoxime systems undergo similar transformations, it might be expected that the excited singlet of benzaldoxjme would not be affected either. Although not conclusive, experimental evidence that the alternative effect of oxygen on the quenching processes could account for the observed result has been obtained and will be discussed later.

Quenching of singlet states by oxygen is not, however, without precedent. Despite the short life-time of a singlet compared to these of triplets, and despite the overwhelming use of oxygen as a diagnostic test for triplets Bowen (167) and Funk (168) have demonstrated that oxygen can effectively compete for the excited singlet of aromatic compounds at rates comparable to the diffusion controlled rates (ca. $\left.10^{10} \mathrm{IM}^{-1} \mathrm{sec}^{-1}\right)$. In most instances, the quenching constants were found to be slightly greater than the calculated maximum diffusion controlled rates. Oxygen was consumed in most cases $(167 \mathrm{a}, \mathrm{c}) \mathrm{p} .38$ ) but there seemed to be no direct relationship between the reduction in fluorescence and the decrease in oxygen content. (Rubrene is an exception). Describing the mechanism of the effect of oxygen on the photochemistry of rubrene, Bowen has suggested that one of the steps (Eq. 39) 
included

$$
\text { Eq. } 39 \text { (1) }{ }^{I_{A}}+\mathrm{O}_{2} \longrightarrow \mathrm{AO}_{2} \text { where } \mathrm{AO}_{2} \text { is an moloxide. }
$$

Both Bowen and Funk have found that radiant fluorescent emission is highly dependent on both the concentration of the fluorescing species and the amount of oxygen in the solution.

With benzaldoxime, analogous processes in which the singlet is appreciably quenched by oxygen, can also occur with reduction in the formation of benzamide. Upon collision between an excitel singlet and a neutral oxygen molecule energy transfer from the excited oxime to oxygen occurs by Eq. 40 which is spin-allowed (169).

$$
\text { Eq. } 40 \quad I_{A}+\mathrm{O}_{2} \longrightarrow 3_{A}+{ }^{I_{0}}
$$

At least it is energetically possible when one considers that the oxygen molecule, whose ground level is a triplet $\left({ }^{3} \Sigma_{\mathrm{g}}^{-}\right)$has the lowest singlet level of energy at 22.5 k.cal. corresponding to transition $\left(\Delta_{g} \longleftarrow^{3} \Sigma_{g}^{-}\right)$, and the lowest excited triplet level at about $98 \mathrm{k}$.cal., corresponding to $\left({ }^{3} \Delta_{4} \stackrel{3}{\longleftarrow} \Sigma_{g}^{-}\right)$transition (170). If the collisional transfer from the benzaldoxime to a neutral oxygen molecule can be represented by process 40, then the amount of energy transfer required is a least $22.5 \mathrm{k}$.cal. in order to promote oxygen from ${ }^{3} \mathrm{Lg}^{-}$to $\Delta_{\mathrm{g}}$. The remaining energy (75 k.cal.) should be sufficient to populate the benzaldoxime triplet 
$\left(E_{T}=49-62\right)$. Singlet-triplet excitation by the process 41 , although spin-allowed by the exchange-mechanism (169, $132 \mathrm{p}$. 252),

Eq. $41 \quad{ }_{A}+{ }^{3} O_{2}^{0} \longrightarrow{ }^{1} A^{0}+{ }^{3} O_{2}^{*}$

would probably be unlikely, in view of the fact that lowest energy level $\left(E_{S}\right)$ of benzaldoxime and that of oxygen $\left({ }^{3} \Delta_{g}\right)$ are almost the same. All other triplet states of oxygen lie above $98 \mathrm{k} . \mathrm{cal}$. with the next lowest one corresponding to ${ }^{3} \sum_{u^{2}}{ }^{3} \Sigma_{\mathrm{g}}^{-}$transition which is $104 \mathrm{k.cal}$. (I70). I 308 and ${ }^{I_{A}}$ represents ground states of the oxygen and the oxime respectively.

Invariably, in preparative experiments, the solution, if irradiated with unfiltered light, eventually turned slightly brown, but when irradiated with filtered light (290mu), both degassed samples and photolyte containing oxygen did not appear to be greatly different. Moreover, the infrared analyses of the photolytes were not different. Therefore, if any adduct is initially formed between the excited benzaldoxime and oxygen, the short-lived adduct must essentially separate to give the benzaldoxime and oxygen possibly through the process (42).

$$
\text { Eq. } 42 \quad{ }^{I_{A}}+\mathrm{O}_{2} \longrightarrow{ }^{I}\left(\mathrm{AO}_{2}\right) \rightarrow \mathrm{A}+\mathrm{O}_{2}
$$

Recently, Just and co-workers (90) have reported the photochemical Beckmann rearrangement of alicyclic ketoximes to 
the corresponding amides and lactams. They suggest that the rearrangement is initiated in the triplet state rather than the singlet. This inference was primarily based on the observed quenching of the rearrangement when oxygen was bubbled through the solution of cyclohexanone orime, despite the lack of photosensitization. From their reports, it is not immediately clear whether any energy transfer occurred upon photosensitization (presumably using an efficient triplet donor) since there would have been no way of demonstrating that energy transfer had taken place by merely analyzing the oxime. There is only one geometric isomer for cyclohexanoneoxime. At the very least, it would have been important to demonstrate that energy was transferred. Whereas with benzaldoxime, owing to the possible existence of two geometric isomer, energy transfer from donor to benzaldoxime could be tested merely by analyzing the relative amounts of $\alpha$ - and $\beta$-isomer. If the triplet level of ethylene $\left(E_{\mathbb{P}}=83\right)$ (171) could serve as a guide to the assignment of the $\mathrm{E}_{\mathbf{T}}$ level of saturated ketoximes, then donors of high Ex value would have been required, rather than the usual sensitizer, benzophenone, with $E_{T}$ value of about 62 k.cal. Moreover, spectroscopic evidence of quenching of phosphorescence of the donor was not demonstrated. No complex is formed between oxygen and $\alpha$-benzaldoxime in cyclohexane. Ultraviolet spectra of saturated solution did not show any difference in the contribution to the absorption spectra other than that due to the oxime. 


\section{Effect of Wavelengths}

In the early stages of this investigation it was found that the yield of benzamides from the benzaldoximes was affected by the use of different filters (89). These observations suggested, therefore, that an important relationship exists between the efficiency of the rearrangement of the oxime and the energy of the exciting source.

The quantum efficiency of the transformation of benzaldoxime was determined at three different wavelengths. The oxime was irradiated in cyclohexane at room temperature in the presence of acetic acid with monochromatic beams of wavelengths $290,253.7$, and $210 \mathrm{mp}$. For irradiation studies at different wavelengths, a standard concentration of the oxime was prepared to absorb more than $99.9 \%$ of the light for the duration of the illumination at all three wavelengths. This was done by measuring the optical density of the photolyte before and after the irradiaticn. At shorter wavelengths, (viz. at 253.7 and $210 \mathrm{~m} \mu$ ) an appreciable amount of light is absorbed by acetic acid and a correction for the amount of light absorbed by the acetic acid is required. At a wavelength of $253.7 \mathrm{~m} \mu$, the incident beam from a low pressuremercury lamp was essentially pure, and the relative absorption of the light by benzaldoxime and acetic acid could be estimated by calculating the relative absorption contributed by both substances to the total optical density of the solution. Separate absorption spectra of these two 
substances were first taken to determine their molar extinction coefficients between 200 and $330 \mathrm{~m} \mu$. At $210 \mathrm{mp}$, however, since the isolated beam had a half band width of $10 \mathrm{mp}$, the relative contribution by benzaldoxime and acetic acid to the total absorption of light was calculated by taking the total area under the curves of these two substances between 200 and $220 \mathrm{~m} \mu$. Although in the presence of 1 equivalent of acetic acid, the relative absorption by acetic acid was negligible, in the presence of 100 equivalents the relative amount was as ligh as $3.78: 1$ in favour of benzaldoxime.

The yield of benzamide was determined by infrared spectrophotometry. The results obtained at different wavelengths are given in Table III. The measured yield of benzamide was shown to be of photochemical origin by analyzing an equal volume of an unexposed solution by the same analytical procedures used for the exposed solution. Invariably no thermal acid-catalyzed benzamide formation could be detected. Even after prolonged standing in neat acetic acid no detectable amount of benzamide was formed from benzaldoxime.

The results in Table III clearly indicate that the efficiency of the rearrangement is highly dependent on the wavelength of the exciting light. At any specified concentration of acetic acid, the yield of benzamide progressively decress with decrease in the wavelength of the exciting 
source. The wavelengths $290,253.7$, and $210 \mathrm{~m} \mu$ correspond to different electronic transition bands of the benzaldoxime and are directly related to the population of the molecules of different energy content. Before the implications of this wavelength dependency can be discussed, it is appropriate to consider the ultraviolet spectrum of benzaldehyde from which the benzaldoxime was originally prepared. (Fig. 13).

In the near ultraviolet spectrum of benzaldehyde, four distinct absorption bands appear. The weakest absorption band appears at the longest wavelength, near $320 \mathrm{~m} \mu$, with a molar extinction coefficient of ten, and is associated with the "forbidden" electronic transition of the non-bonded electrone localized in the orbital on the oxygen atom, to the anti bonding : $\tau^{*}$ orbital of the benzaldehyde molecule. This is generally termed the $n \pi^{*}$ or $R$ band of carbonyl compounds $(107,172,174)$. The three other bands between 180-300m $\mu$ have been attributed to the corresponding $\pi \pi^{*}$ transition band of the benzene molecule, which is shifted into the normally accessible region of the ultraviolet spectrum, due to the participation of the carbonyl function with the benzene ring. The three $\pi \pi^{*}$ bands in benzaldehyde have been identified as those corresponding to $\left(A_{19} \leftarrow B_{2 \mu}\right.$ or ' $L_{b} \leftarrow A$ )

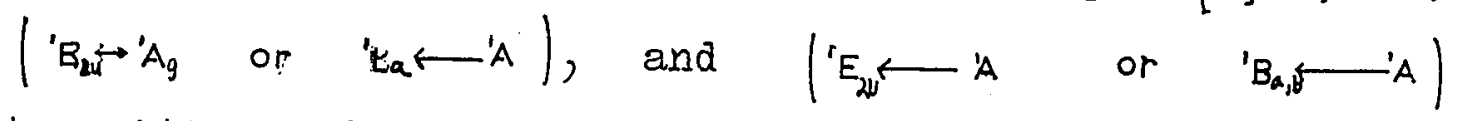
transitions of the benzene molecule in the decreasing order of wavelength. The weak absorption band, appearing at about 270-290m and showing fine vibrational structure with molar 


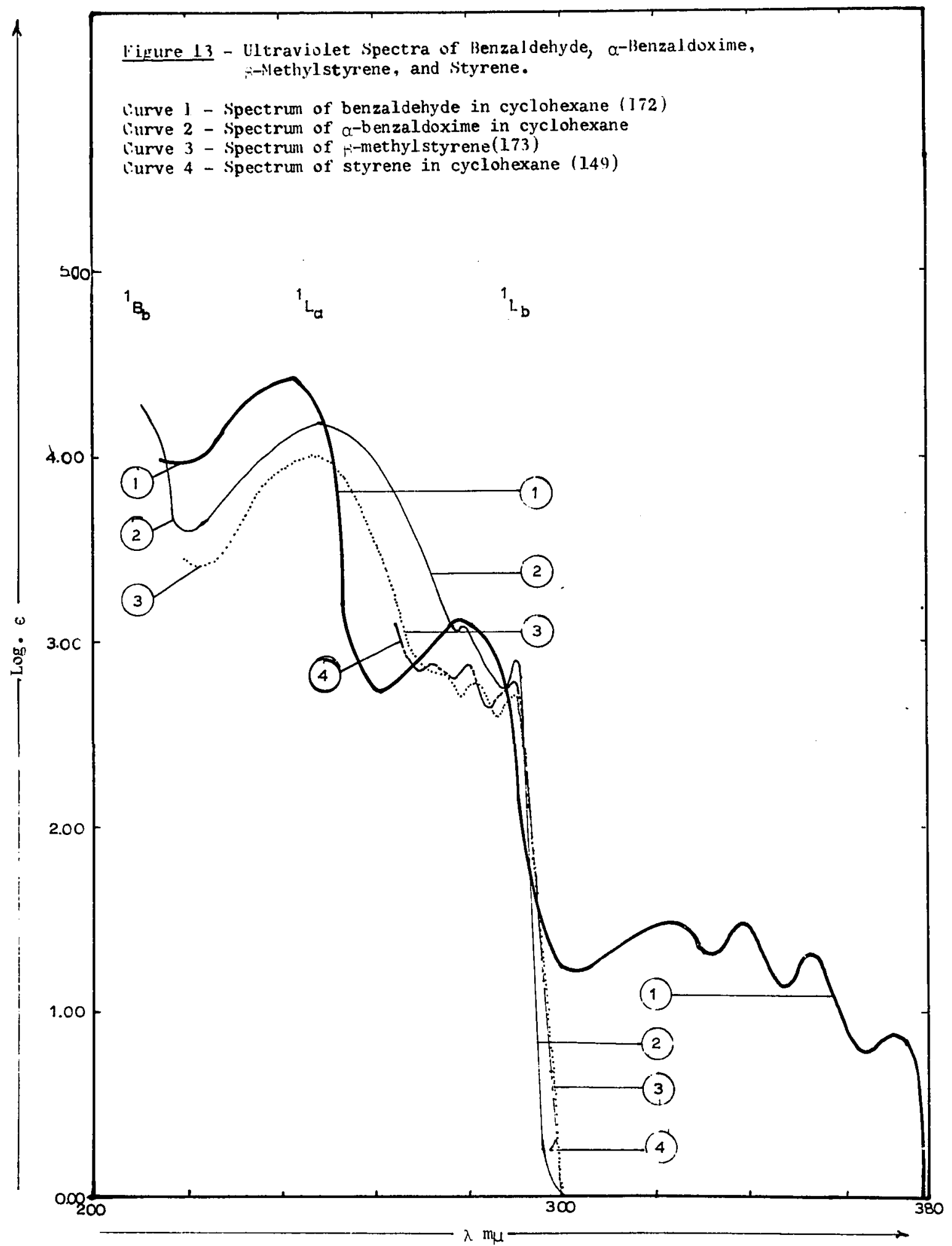


extinction coefficient of 1000-2000, is regarded as a transition corresponding to the symmetry-forbidden ' $\mathrm{L}_{b} \longleftarrow \mathrm{A}_{\mathrm{g}}$ transition of the benzene. 'L $L$ ' $A g$ band is generally referred as the B-band, Benzenoid band, or the secondary band. (175, 176).

Oximation of the carbonyl function of benzaldehyde leads to the complete disappearance of original $n x^{*}$ band. This is general for aromatic and conjugated carbonyl compounds*. Even with saturated ketones, such as acetone, the $n \pi^{*}$ band originally present at $275 \mathrm{~m} \mu(E=15)$ disappears upon oximation and intense absorption is observed at $190 \mathrm{~m} \mu \quad(E=5,000)$ (ref. $107 \mathrm{p} .103,177,184, \mathrm{p} .204,173)$ comparable to that of ethylene which absorbs at $185 \mathrm{~m} \mu(E=8,000)$.

When benzaldehyôe is converted to benzaldoxime or benzilidene methyl. . amine, the spectra are almost superimposed: on the ultraviolet spectra of styrene or $\beta$-methyl styrene. The ultraviolet spectra of benzaldoxime and benzilidene-anil type compounds consist of (a) a rather weak band with vibronic structures at the longest wavelength region (275-300mu, $(\mathbf{E}=1,800),(b)$ an in̈tense band at about 250mp ( $E=14,000)$, and (c) another very intense band continuing toward the vacuum ultraviolet region. On the basis of theoretical and experimental analysis of the ultraviolet spectrum of styrene and on the close similarity of the ultraviolet spectrum of benzaldoxime to that of styrene, Kubota

* K. Yoshida, D.R. Kearne (I79) recently reported that oximation of fluorenone removed the $n \pi^{\prime}$ transition of the ketone. 
(178) has assigned the three regions of the spectra of benzaldoxime and benzilidene-methyl amine as those corresponding to ' $L_{b}, L_{a}$ and ' $B_{b}$ transitions of tile benzene. The designation used by Kubota is the same as Platt's Notation for the styrene transition. Moreover, simple molecular orbital calculation and observed results of benzilidene methylamine have been regarded by Kubota as being in good agreement.

Compared to benzene which shows no 0-0 absorption of the $\left({ }^{I} I_{b} \longleftarrow I_{A}\right)$ type (2I2) because of its symmetry, substituted benzenes exhibit this transition. In styrene, the 0-0 electronic transition is observed at $287.7 \mathrm{~m} \mu$ in the vapour phase $(149,180,181)$. In cyclohexane solution(180), the 0-0 line of styrene is shifted to $290.5 \mathrm{~m} \mu$. Referring to Fig. 13 the 0-0 transition band of the benzaldoxime is assigned to the vibronic structure at $291.5 \mathrm{mp}$ which is almost coincident with that of styrene and $\beta$-methylstyrene.

As further support for the assignment of the $\pi \pi^{*}$ transition band in the ultraviolet spectra of benzaldoxime, the change in the absorption maximum underwent a very small bathochromic shift when spectra were taken in polar solvents. The absorption maximum at $250 \mathrm{~m} \mu$ and $291.5 \mathrm{~m} \mu$ in cyclohexane shifted toward the "red" by only $2 m \mu$ in methanol and water. Kubota also found the shift of the same order of magnitude when spectra were taken in n-heptane (178). These bathochromic shifts are comparable to the small "red" shift of 
$3 m \mu$ in the $\pi \pi^{*}$ transition band of aromatic ketone (182). The corresponding $n \pi^{*}$ transition bands in the ketones are known to undergo a hypsochromic shift to a much larger extent than the bathochromic shift of the $\pi \pi^{*}$ bands. As a further support to the assignment of the $\pi \pi^{*}$ band of the oxime, the change in solvents from cyclohexane to methanol did not totally blur out the strong vibronic structure at $2.91 .5 \mathrm{~m} \mu$ (cyclohexane) and remained essentially sharp, unlike those observed for structure of the $1 \pi \pi^{*}$ transition band (154, p. 182). Even in neat acetic acid, vibronic structure was still visible in the spectrum.

From the foregoing, the light induced transformation of benzaldoxime to benzamide can be considered a consequence of the $\pi \pi^{*}$ excitation of the oxime. Recently, Just and coworkers (90) who have reported similar rearrangements of alicyclic ketoximes to their corresponding amides, have suggested that the primary photo-excitation of the step is a $\pi \pi^{*}$ or a highly forbidden $n \pi^{*}$ transition.

The dependency of the quantum efficiency of the rearrangement on the wavelength of the exciting light can be interpreted in one of several ways. First, it is important to consider whether the observed decrease in the quantum yield of benzamide is a result of secondary photochemical reactions of the initially formed benzamide since such a destruction of benzamide could readily account for the 
observed decrease in quantum yield at lower wavelengths. Amides, whether of aliphatic or aromatic types, are known to be sensitive to ultraviolet light leading to rearrangement, as exemplified by the Photo-Anilide rearrangement reported by Elad (16a) and the photolysis of aliphatic amides in solution to carbon monoxide, and hydrogen, primary amines, (183a). In the vapour phase, in addition to the products observed in solution, nitrites and ammonia are also formed $(183 b)$. At $290 \mathrm{~m} \mu$, the decomposition by a secondary photochemical reaction of benzamide could be excluded since a "blank" solution containing only benzamide was unaffected. This might have been expected since the relative molar extinction coefficients of benzaldoxime and benzamide at $290 \mathrm{~m} \mu$ is about $100 / 1$, and since the amount of benzaldoxime remaining after irradiation would have been far in excess of the benzamide that was formed. Even the large conversion in neat acetic to the extent of $27 \%$. would not lead to an appreciable amount of absorption by the benzamide. At $253.7 \mathrm{~m} \mu$, the similar result would be expected since the amount of light absorbed by benzamide, relative to that absorbed by the starting material, would be insignificant. The differences in the quantum yields at $253.7 \mathrm{~m} \mu$ and $290 \mathrm{~m} \mu$ are not due to decomposition of benzamide. For example, one can compare the results obtained under the same experimental conditions in the presence of acetic acid. At $290 \mathrm{~m} \mu$, the conversion quantum yield (0.006) corresponds to $1.7 \%$ conversion of benzaldoxime to benzamide. Since the relative 
molar extinction coefficient of benzaldoxime and the amide at $253.7 \mathrm{~m} \mu$ is $24 / 1$, the amount of light absorbed by the benzamide would have been less than 0.1\%. Further, even if one assumes that the rearrangement in neat acetic acid is $\phi_{B z}=0.058$ for this amount of conversion, the absorption of light by benzamide would not be sufficient to make a difference six ffold between the quantum yields at 290 and $253.7 \mathrm{~m} \mu$. If the same argument is extended to the result obtained at $210 \mathrm{~m} \mu$, the amount of light absorbed by benzamide could be significant since the ratio of the molar extinction coefficients of benzaldoxime and benzamide at $210 \mathrm{~m} \mu$ is only about 2.8 to 1 . As benzamide is formed, a gradual decomposition due to absorption of light could occur. If one assumes again that the quantum yields $\left(\phi_{B z}=0.026\right)$ at $290 \mathrm{~m} \mu$ and at $210 \mathrm{~m} \mu$, under the same experimental conditions in acid were the same, the amount of light available to both substances could be absorbed by benzamides to the extent of 3-4\%. Although no quantum efficiencies of the decompositions of amides are available in the literature, if the efficiency is about 0.5 , comparable to the decrease of aryl.esters encountered in the Photo Fries reaction (0.65) (15a), one might expect to observe total destruction of the benzamide. Therefore, attachment of real significance to the observed quantum yield at $210 \mathrm{~m} \mu$ might require some reservation.

Since the intensity of the incident light was monitored using the ferrioxalate actinometer solution, the value of the quantum yield $(\phi=1.28)$ for the actinometer solution was 
used in the calculation of the light absorbed by the photolyte. This value of 1.28 was obtained by extrapolation of the data reported by Parker and Hatchard (184) based on 12 values of quantum Jields between $254 \mathrm{~m} \mu$ and $578 \mathrm{~m} \mu$. Since the electronic transition band of the actinometer solution gradually increases in intensity from the visible region of the spectrum to the vacuum region of ultraviolet spectrum, no abrupt change in the value is expected. Moreover, there appeared to be a very gradual change in the quantum yield reported with decrease in wavelength. It slowly reached a plateau in the region of $254 \mathrm{mp}$. A rough estimation of the value at $210 \mathrm{~m} \mu$ (see experimental detail) was made for the ferrioxalate solution and the yield was found to be 1.4. Quantum yields calculated using a ferrioxalate quantum even yield as high as 2.0 would not invalidate the arguments so far presented.

Another factor which might have contributed to the low quantum yield observed at $210 \mathrm{~m} \mu$ is the decomposition of acetic acid. In its absence the production of benzamide is very poor. Decomposition of acetic acid by a Norrish-Type I process has been observed, and the sum of quantum yields for the primary processes has been estimated to be in theorder of 0.5 (185). At $210 \mathrm{~m} \mu$ in the presence of acetic acid $(0.0071$ $\mathrm{M}^{\cdots}$ ), the quantum yield $\phi \approx 0.001$ at $210 \mathrm{~m} \mu$ whereas at $290 \mathrm{~m} \mu$, $\phi_{B 2}=0.006$. However, the amount of light absorbed by acetic acid relative to that absorbed by benzaldoxime, was small. 
On the other hand, in the presence of 100 equivalents of acetic acid, the amount of light absorbed by the acid would have been as high as 25\%. Assuming the quantum yield of acetic acid decomposition to be 0.5 , the reduction in the concentration of acetic acid would not exceed $3 \%$ of the initial amount. Acid reduction alone would not have reduced the quantum yield by a factor as large as 13 between 210 and $290 \mathrm{~m} \mu$.

Excluding some of the experimental factors which could be responsible for the decrease in the quantum efficiency of the rearrangement, the results must therefore be due to the nature of the excited molecules or a consequence of the difference in the energy content of the different excited states. The exposure of benzaldoxime to light of $290 \mathrm{~m} \mu$, 253.7m $\mu$, and $210 \mathrm{~m} \mu$ corresponds to excitation of the three different electronic transition bands of benzaldoxime.

In describing the mechanism of photochemical reactions, there is general agreement that (a) most primary processes which occur from an electronically excited state are initiated by the molecule in its lowest vibrational level of the first excited state, and (b) excitation of a molecule to a higher electronic level or levels is subsequently followed by an extremely rapid, radiationless, decay, by internal conversion (within $10^{-13} \mathrm{sec}$ ) to the lowest vibrational level. Internal conversions from higher levels to the lowest vibrational state occurs in both the singlet and triplet manifold. In 
view of this, the rearrangement of benzaldoxime to benzamide probably occurs from the lowest vibrational level of an excited singlet state. It is of interest to point out that the most efficient conversion is observed when benzaldoxime is excited at $290 \mathrm{m \mu}$, a wavelength which corresponds almost to the 0-0 transition of the lowest min electronic band $\left({ }^{I} I_{b} \leftarrow{ }^{I} A_{g}\right)$ observed at $291.5 \mathrm{~m} \mu$. At this wavelength, the amount of vibrational energy in excess of the lowest level of the first excited state would be expected to be very small. This observation strongly suggests that the photochemical Beckmann rearrangement of benzaldoxime occurs from the lowest vibrational band of the $S_{I}\left(L_{b}\right)$ and that any factor which decreases the population of this level can result in a lower quantum yield. One such possibility is the very rapid internal conversion from the first excited state above the 0-0 level ( $L_{b}$ ) to the higher vibrational levels of the ground state $\left(S_{0}^{\nu^{\prime}}\right)$ before the establishment of a vibrational equilibration with the environment of an excited molecule. Since the photochemical rearrangement of benzaldoxime does not appear to proceed to the corresponding benzamide by the same pathway available to the thermally activated, acid-catalyzed reaction in the ground state, the rearrangement from the $S_{0}^{\nu^{\prime}}$ level is an unlikely course for the reaction. Moreover, such a pathway would be facilitated by collisions with solvent molecules leading to a rapid vibrational relaxation of the $\mathrm{S}_{0}^{\nu^{\prime}}$ state. Therefore, this mode of dissipation of energy is an improbable pathway and does not account for the 
observed wavelength dependency of the reaction. On the other hand, it could be explained by considering the possibility of an appreciable direct intersystem crossing from levels of the singlet states, above the 0-0 level of $S_{1}$, to the corresponding $\pi \pi^{*}$ triplet states before complete internal conversion of the upper $\pi x^{*}$ singlet state to the lowest $S_{I}$ level occurs. This process cannot be excluded in view of the fact that photochemical geometrical isomerization is more efficient than the structural rearrangement itself and is observed to take place when irradiated at all wavelengths $313.3,290,253.7$ and 210 $m \mu$. If, in fact, a singlet-triplet intersystem crossing does occur efficiently from higher vibrational levels above the zero vibrational level of $s_{1}$ or from higher electronic levels, then a corresponding decrease in the population of the lowest $\mathrm{S}_{1}$ level would occur, and thereby reduce the quantum efficiency of the rearrangement. Parker and Hatchard (186), who observed. an increase $(10-20 \%)$ in the ratio of $\frac{\phi_{p}}{\phi_{f}} \quad\left(\phi_{p}\right.$ is the quantum yield of phospiorescence, $\phi_{f}$ is the quantum yield of fluorescence) when excitation of eosin was changed from $546 \mathrm{~m} \mu$ to $436 \mathrm{~m} \mu$, have attributed the wavelength dependency of $\frac{\phi_{p}}{\phi_{f}}$ to an appreciable extent of direct singlet-triplet intersystem crossing at the lower wavelengths. This phenomenon was observed for eosin at room temperature in solution but a similar wavelength dependency of $\frac{\phi_{p}}{\phi_{f}}$ has been also observed by Bauer and Baczynski (187) for fluorescein at $77^{\circ} \mathrm{K}$ in rigid media. 
Another important possibility and a more probable mechanistic pathway which could account for the wavelength dependency of the transformation, is the increase in relative importance of other modes of reaction pathways from a higher excited state above the lowest vibrational level of $S_{I}\left(L_{b}\right)$. At different wavelengths, the energy of an excited state becomes entirely different and this difference in energies could readily lead to entirely different photochemical products. The three different wavelengths at which the wavelength dependence was investigated, viz., at $290,253.7$ and $210 \mathrm{mp}$ are equivalent to $98.0,116$, and $139 \mathrm{k} . \mathrm{cal}$. of energy, respectively, (188). Upon excitation, especially at lower wavelengths, the localization of the absorbed energy in one of the weakest bonds could readily lead to a homolytic cleavage. The irradiation at $290 \mathrm{~m} \mu$ of benzaldoxime produces, in addition to benzamide, asmall amount of benzaldehyde. Although no benzonitrile was detected in the photolyte when analyzed by infra-red, small amounts might also be formed. RecentIy, Just (190) reported that irradiation of the oxime of 17B-acetoxy-6-formyl-3-methoxy-androsta-3,5-diene in methanol at $253.7 \mathrm{~m} \mu$ afforded, in addition to $17 \%$-acetoxy-6formy 1-3,3-dime thoxy-androsta-5-ene, 17B-acetoxy-6-cyano-6methoxyl-androstan-3, 5-diene. Hata (886), who studied the vapour phase photolysis reaction of 2-picoline-N-oxide, observed that irradiation with light at $254 \mathrm{~m} \mu$ (117 k.cal.) in the $\pi \pi^{*}$ region of the electronic transition band led to 2-picoline which had been deoxygenated. Irradiation at 
higher wavelengths in the ${ }^{\pi^{*}}$ region with a light beam of $327.6 \mathrm{~m} \mu$ (82.3 k.cal.), afforded 2-pyridinyl methanol instead. The genesis of 2-pyridinyl methanol which is considered to be intramolecular, demonstrates the importance of the energy of the exciting source. Moreover, the production of decomposition products such as carbon dioxide, hydrogen and oxygen, was also greater at $254.3 \mathrm{~m} \mu$ than at $327.6 \mathrm{~m} \mu$. However, in this instance, it must be recognized that the difference in nature of the excited state, rather than the difference in energy content of the exciting energy, might be more important since irradiation at 254.3 and $327.6 \mathrm{~m} \mu$ corresponds to the $\pi \pi^{*}$ and $n \pi^{*}$ respectively, of the electronic transition band. In benzaldoxime, the $\mathbb{N}-0$ bond is likely to be the weakest bond (6I k.cal.), (188) well below the energy of the irradiating sources $(290 \mathrm{~m} \mu=98.0)$ and is susceptiule to bond cleavage, especially at higher energy of the exciting light. In the extreme case where $x$-rays have been used to irradiate benzophenone oxime, rather than isolating any benzanilide resulting from a Beckmann-type rearrangement, diphenylmethyl amine was isolated as one of the reaction products (189). Although no report of photochemical Beckmann-type rearrangements of aromatic ketones has ever been published, it has been shown in our laboratory that p-methoxyacetophenone oxime can be rearranged in the same manner as that observed for benzaldoximes. 
E Effects of Substituents, Solvents, and Acids

Spectroscopic evidence, as well as chemical evidence for the formation of 3-phenyloxazirane, has already been presented. That the intermediacy of 3-phenyloxazirane could lead to benzamide has been suggested by Paquette (I9I) who demonstrated that treatment of the $\mathrm{N}$-chloro derivative of 0 -benzylhydroxylamine with base affords benzamide, and Christmann and Horner (192) who also isolated benzamide from the reaction of benzaldenyde and hydroxylamine-0-sulfonic acid. Experimental conditions in both instances were under alkaline media, indicating that 3-phenyloxazirane, if formed, is converted to benzamide. The isolation of benzamide by Christmann has a direct bearing on the photochemical conversion to benzamide, since Schmitz and co-workers (110c) have reported that the use of the same reagents as Christmann's, under appropriate reaction conditions, afforded the corresponding 3-phenyloxazirane which could be trapped with suitable reagents. Although the oxaziriane itself appeared to be too unstable for proper characterization, various iv-methyl analogues of 3-phenyloxaziranes have been synthesized similarly; and in certain instances, have been isolated and fully characterized $(I I 0 e)$. Accompanying the formation of the oxaziranes were their corresponding $\mathbb{N}$-methyl benzamides but the corresponding formanilides were never isolated. Schmitz hai suggested that these $\mathbb{N}$-methyl-3-phenyloxaziranes and the $\mathbb{N}$-methyl amides are formed from the same intermediate 109, but conclusive evidence 
has not been provided that the oxazirane 110 is rearranged to the corresponding $\mathbb{N}$-methyl amides 111 (Reaction 28).

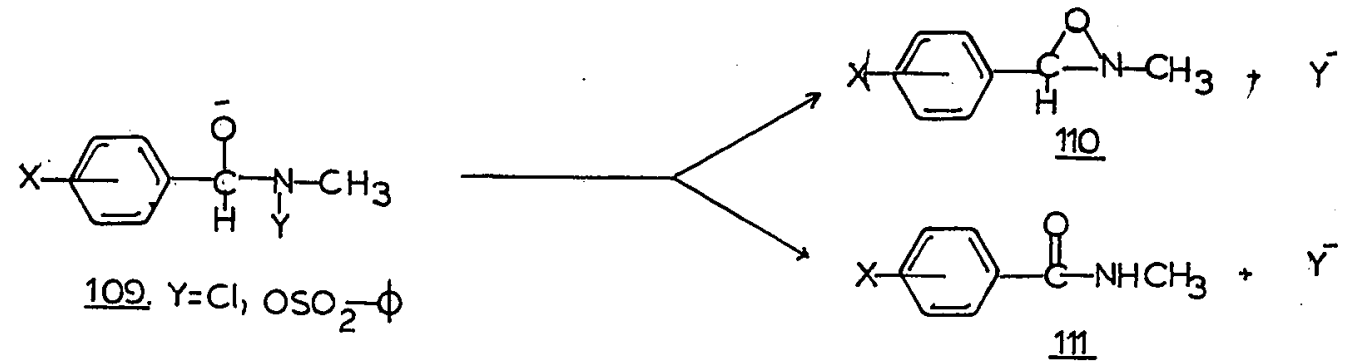

Reaction 28

There is however, an adequate reason to believe that, in fact, 2-methyl-3-phenyloxaziranes can rearrange thermally to N-methyl benzamides. Recently, Hansen and Jeslev (76) have conclusively established that irradiation of 2,6-dimethyl-4-bromobenzaldehyde-N-methyloxime affords the corresponding oxazirane. Furthermore, they have reported that irradiation of N-methylp-chlorobenzaldoxime 112 yields the corresponding p-chloro$\mathbb{N}$-methylbenzamide 113 , thus providing evidence for the

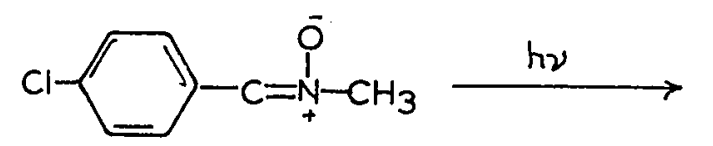

$\underline{112}$

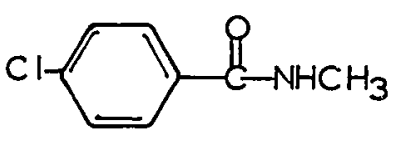

$\underline{113}$

Reaction 29

rearrangement of $\mathrm{N}$-methyl-3-phenyloxazirane to $\mathrm{N}$-methylbenzamide. Formation of the corresponding formanilides was not reported either. The similar photochemical transformation of the N-methyl unsubstituted nitrone has been reported by Shindo (113). Benzaldehyde, which has been detected in the 
photolyte of benzaldoxime, has also been observed on irradiation of $N, \alpha$-diphenylnitrone which is known to decompose via the 3-diphenyloxazirane $(68,113)$.

The interpretation of the results in benzaldoximes are, however, grossly complicated by the formation of an intermediate, 3-phenyloxazirane, since the quantum yield measurements reflect only the over-all efficiency of the rearrangement rather than the primary yields. The intervention of the intermediate not only prevents one from attributing factors which govern the primary processes of the reaction which in turn leads to the intermediate, but also restricts assigning factors controlling the reaction of the electronic ground state of the intermediate. This eventually leads to the final product. In view of this complication in the interpretation, the discussion of the effects of substituents, solvents, and acids will be treated separately and a rationale for the results will be presented where possible.

I Effects of Substituents on the Excited Singlet State of Benzaldoxime

Preliminary investigation of the photoconversion of various benzaldoximes to benzamides indicated that the introduction of substituents into the aromatic ring decreased the yields and, in certain instances, benzamides were isolated either in extremely low yields or not at all (89). Quantum 
yields of p-substituted benzamides from the corresponding benzaldoximes were determined at $290 \mathrm{~m} \mu$ in the presence of a standard concentration of acetic acid and the results are recorded in Table IX. A logarithmic plot of the quantum yields against sigma functions $\left(\sigma_{p}, \sigma_{p}^{*}, \sigma_{p}^{\circ}\right)$ (108) is shown in Fig. 14. The quantum efficiency varied between 0.009 and 0.025 with a maximum recorded for benzaldoxime. The quantum yields for benzaldoxime and p-fluorobenzaldoxime are similar. The relationship between the quantum yield the rearrangement and the sigma functions parallels that of the yield previously reported by Amin and deMayo (89).

Two new amides which were not detected previously from the photochemical reaction were found. p-Fluorobenzamide was shown to be formed from p-fluorobenzaldoxime, and was identified by comparison with an authentic sample: $R_{f}$ values, melting points, infrared and ultraviolet spectra of the reaction product were identical with those of the chemically prepared material. p-Methoxyformanilide, in addition to panisamide and polymeric material, was also isolated from panisaldoxime, and was identified by comparison with an authentic sample. The quantum yield of p-methoxyformanilide was slightly greater than that of the p-anisamide. (See Table IX). The p-methoxyphenylmoiety had a migratory aptitude exceeding that of hydrogen by a factor of two. It was demonstrated that prolonged irradiation through quartz led to a gradual destruction of the initially formed amides as a 
secondary reaction. This accounts for the failure of the previous worker (89) to isolate these two amides. It was found that careful filtering of the light to prevent appreciable absorption by the amides: generally prevented their total destruction.

That the rearrangement of the p-subitituted benzaldoximes was photochemically induced was shown by the failure of unexposed samples to yield amides, when subjected to experimental conditions otherwise identical to those for the photolyte.

Spectral data for p-substituted benzaldoximes are given in Table VIII. The general contour of the absorption spectra of the p-substituted oximes do not differ appreciably from those of benzaldoxime (See Fig. 15). The 0-0 singlet energy bands of the oximes lie between 94.8 and $99.0 \mathrm{k.cal}$. The largest difference between the absorption maxima of a substituted benzaldoxime and that of benzaldoxime was $20 \mathrm{~m} \psi$ for the ha band. The largest molar extinction coefficients of the substituted benzaldoxime was 1:51 that of benzaldoxime, for the 'La transition band. Most benzamides exhibit a cutoff at about 290m $\mu$ except for p-methoxyformanilide $\therefore$. which did not fall off until $325 \mathrm{~m} \mu$. The molar extinction coefficients of p-methoxyformanilide at $290 \mathrm{~m} \mu, 297 \mathrm{~m} \mu$ and $302 \mathrm{~m} \mu$ are 1910 , 1300 , and 382 respectively.

Iarge scale irradiation of p-methoxyacetophenone oxime through a Corexvycor filter, in the presence of acetic acid, 
afforded a mixture of $\mathrm{N}, \mathrm{p}$-anisylacetamide, N-methylanisamide, and p-anisamide in the ratio of 10:5:1. The amides were identified by comparison of their physical constants and spectral data with those of authentic samples.

Quantum yield measurements of the three amides are recorded in Table $X$. Both the yields and the quantum efficiencies of the formation of $\mathrm{N}$-p-anisylacetamide and $\mathrm{N}$ methylanisamide are almost equal: p-anisamide having the smaller value in each case. p-Anisamide was a demethylated product. The sum of the quantum yields of the amides,0.013, is very similar to the value of 0.012 , obtained for p-anisaldoxime which rearranged to both p-methoxyformanilide and panisamide under similar conditions. The importance of acid in effecting the conversion was also demonstrated. Quantum yields measured under protonic and aprotic conditions differed by a factor of about 13, close to that for benzaldoxime under similar conditions.

The TIC-UV spectrophotometric nethod employed for quantitative estimation of the amides is reliable since almost complete recovery of the amides can be made by using the appropriate solvent system for the extraction from silica gel.

Under the experimental conditions employed, the amount of light absorbed by the p-substituted benzamides at $290 \mathrm{~m} \mu$ is not appreciable except for p-methoxyformanilide. Because of 


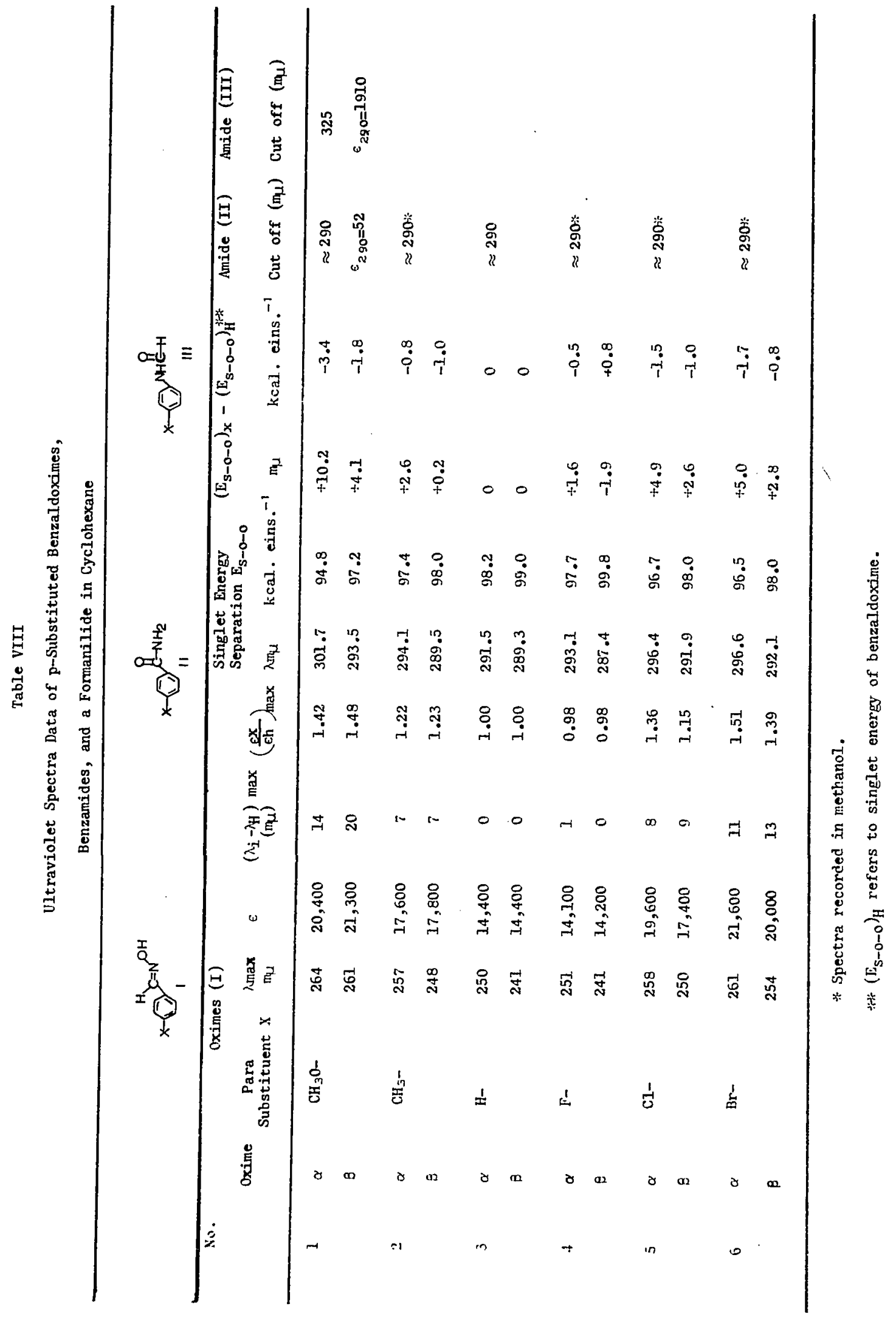




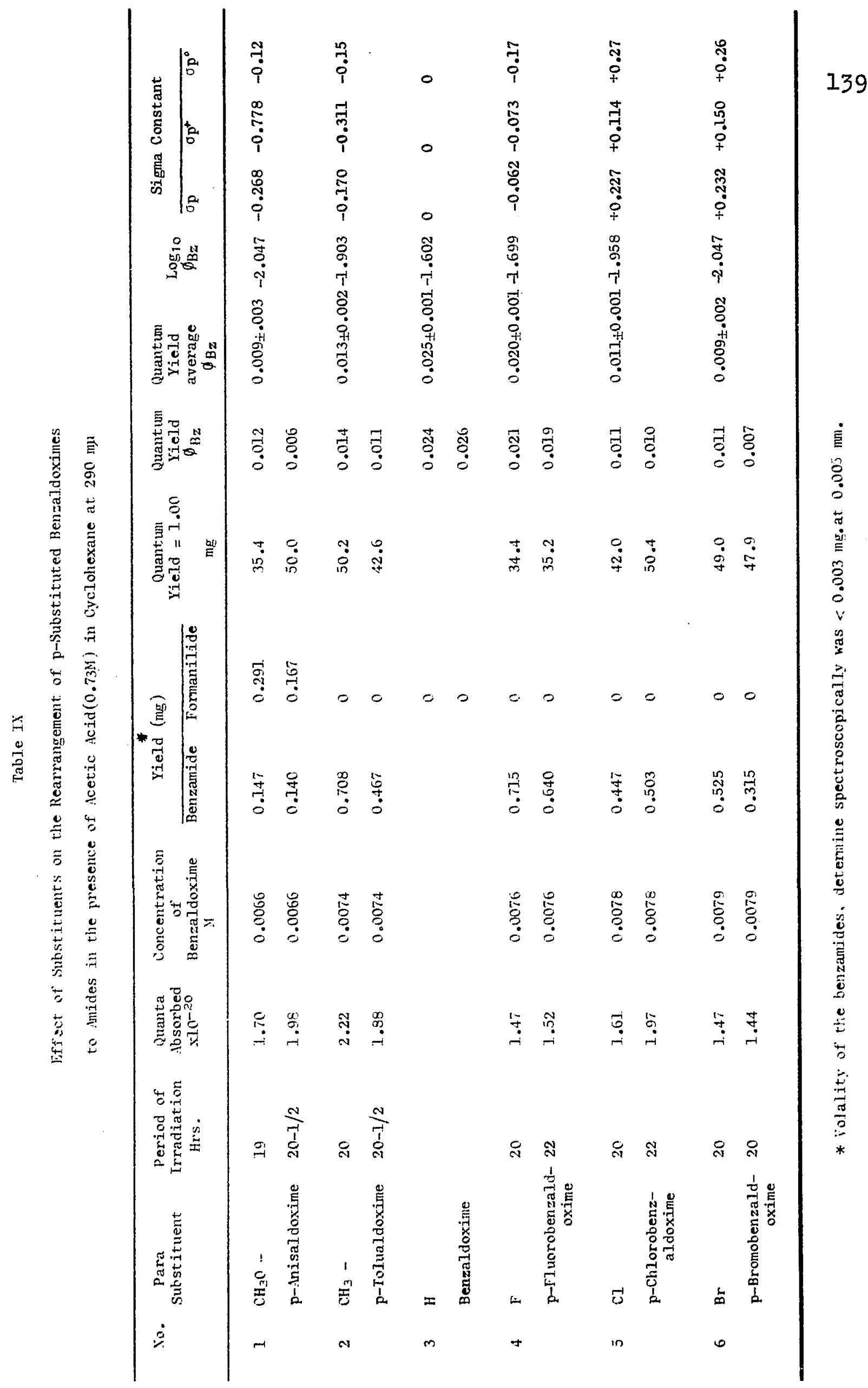




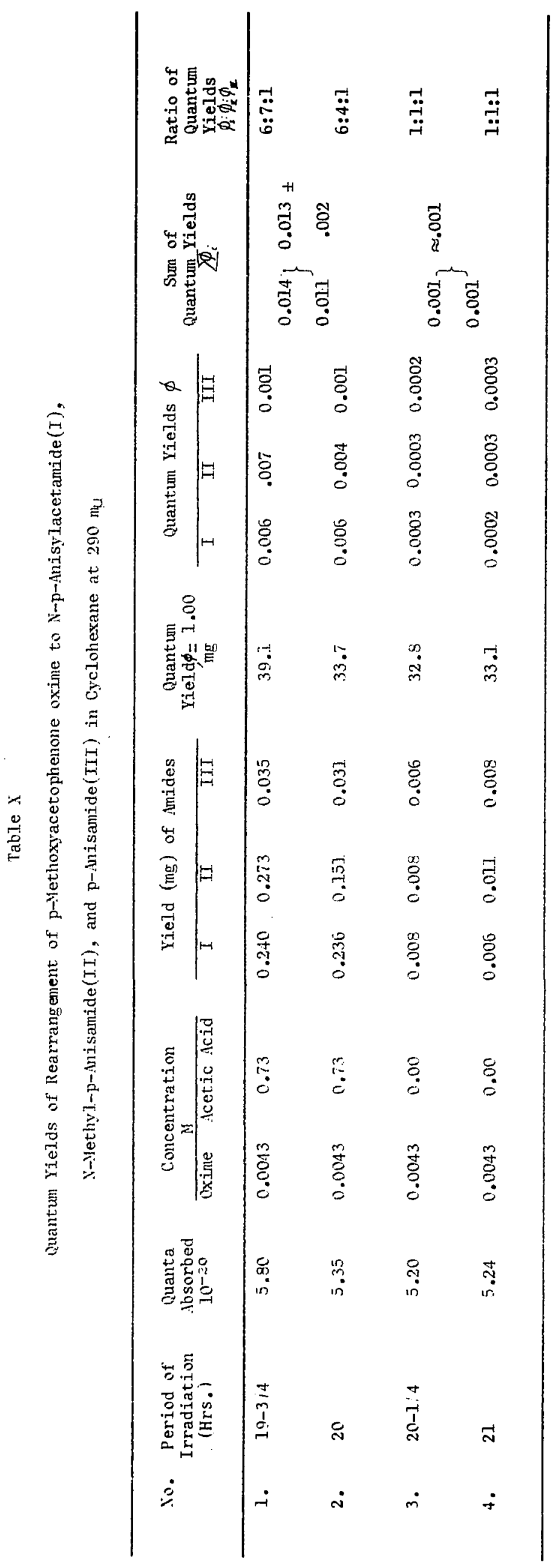




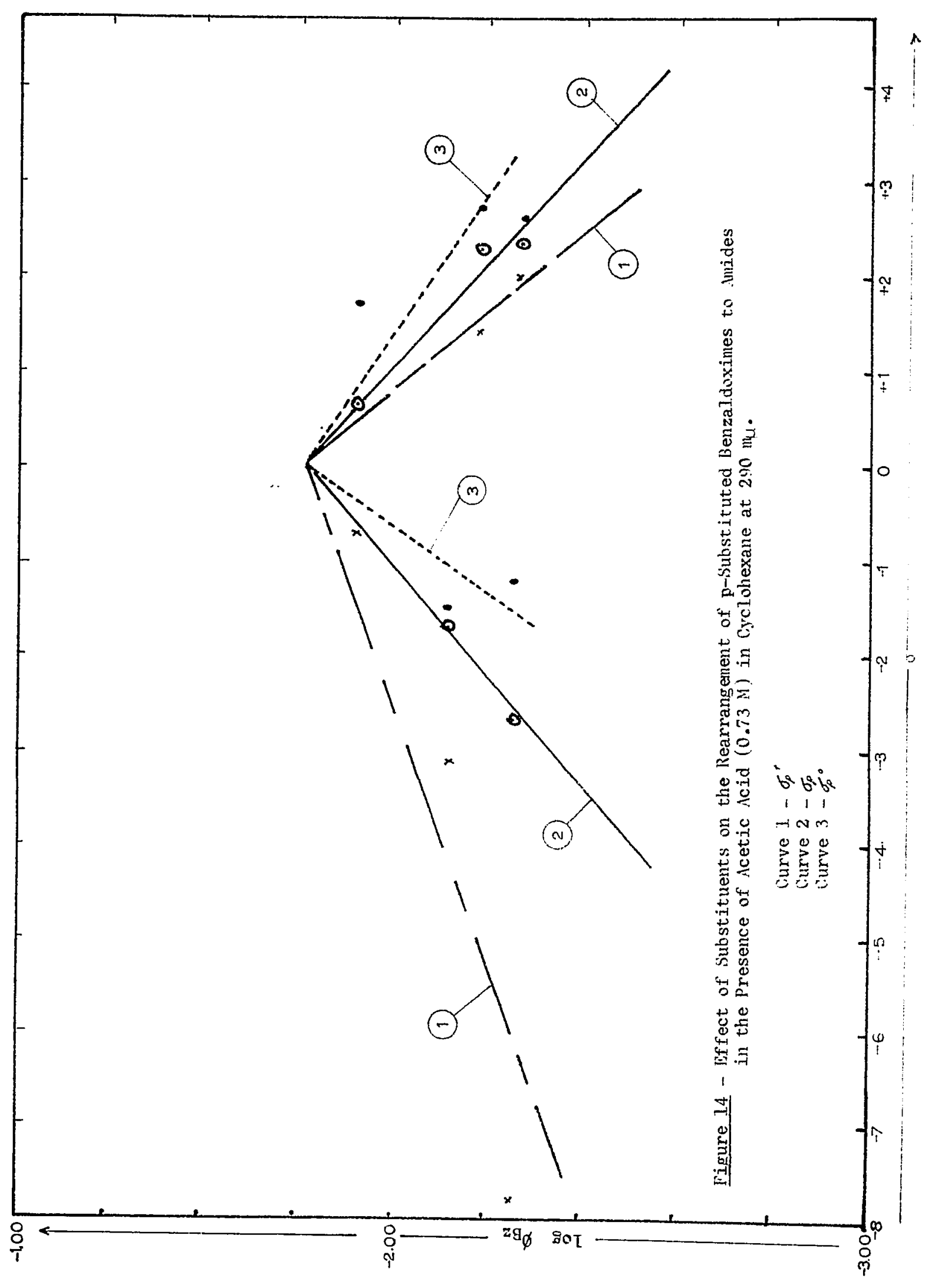




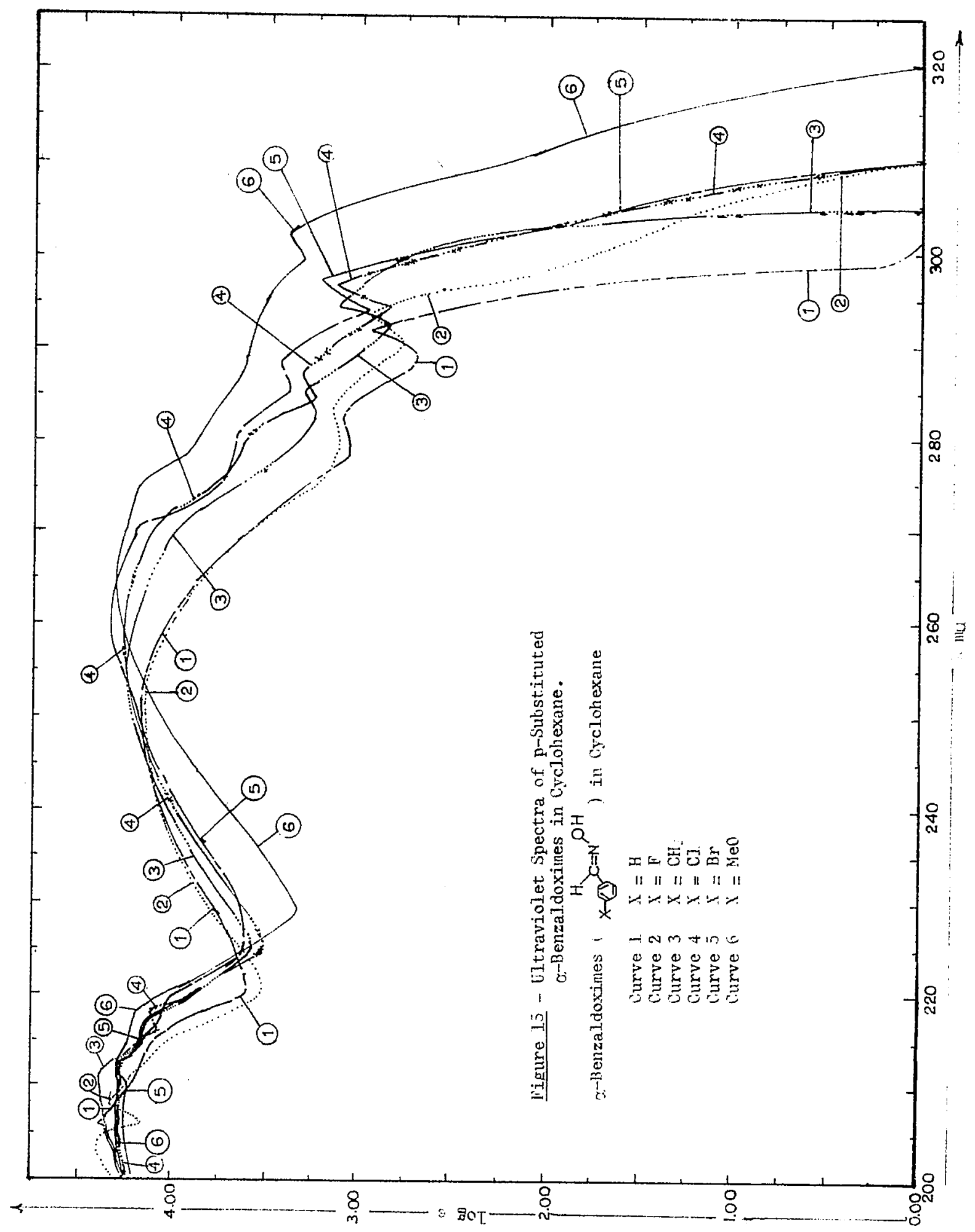


the relatively high molar extinction coefficient of this compound at $290 \mathrm{~m} \mu$ ( $\mathrm{E}=1910)$, about $15 \%$ of the exciting 7.ight could have been absorbed during the irradiation. Invariably, the photolyte of p-anisaldoxime was slightly brown, indicative of photolytic decomposition. In one instance, upon exposure to a specified amount of light, it was observed that the relative amount of p-methoxyformanilide to p-anisamide decreased when compared to conditions in which light was absorbed. Therefore, the quantum yield of 0.012 is considered to be a more accurate value than 0.006 for the conversion of p-anisaldoxime to the amides. Because of the isolation of two amides from the photolytic conversion of $p$ anisaldoxime, the photolytes of the p-substituted benzaldoximes were carefully scrutinized for the presence of $p-$ substituted formanilides as well as for the corresponding $p$ substituted benzamides. Except for p-anisaldoxime, p-substituted benzamides were found to be the only amides formed. Apparently, the introduction of the p-methoxy group is sufficient to divert the rearrangement process from reaction pathways common to most of the benzaldoximes.

For p-benzaldoximes, the effect of substituents on the ultraviolet spectra gave reason to believe that substituents could have a direct influence on the rearrangement processes. Except for p-fluorobenzaldoxime, the p-substituted benzaldoximes show a noticeable bathochromic shift relative to that of benzaldoxime in the absorption maxima of the ${ }^{I_{I_{b}}}$ and the 
${ }^{I_{I}} I_{a}$ electronic transition bands. The intensification of absorption upon substitution accompanied the shift of these maxima. The largest shift and the largest absorption intensity in the ${ }^{I_{I_{b}}}$ and the ${ }^{{ }^{I_{I}}}$ were observed for p-anisaldoxime (see Fig. 15 and Table VIII). The position of the absorption bands in the $200 \mathrm{m \mu}$ region was found to be less sensitive to substitution.

Although a bathochromic shift is observed for the ${ }^{{ }^{1}} I_{a}$ transition with respect to that of benzaldoxime, that undergone by the ${ }^{I_{I_{b}}}$ band representing the band of the first excited state, is considerably smaller. The largest difference in the position of the 0-0 singlet transition was found for p-anisaldoxime $(3.4 \mathrm{k} . \mathrm{cal}$.$) . For oximes substituted in the$ para position by methyl, fluorine, chlorine, and bromine, the energy differences in the 0-0 levels of these compounds are less than $2 \mathrm{k}$.cal. Since the energy contert of the oximes would generaliy be expected to be defined by the lowest vibrational level of the first excited state from which rearrangement occurs, a difference in the energy content of this order of magnitude, between the various excited benzaldoximes, is unlikely to be a controlling factor in the observed decrease in the quantum efficiency. All oximes were irradiated at $290 \mathrm{~m} \mu$ and this led to the excitation of the oximes in the ${ }^{I_{I_{b}}}$ transition band.

Previously (89) had been suggested that substituent effects may be interpreted from the point of view that they 
modified the nature of the excited states, as observed in the photoreduction of substituted benzophenones (193). The differences in reactivity of substituted benzophenones inclusive of photoreduction (193,194), oxetane formation (195197), and pinacolization (198-199) have been attributed to alterations in the position of the lowest triplet energy states of different electronic configurations, $n \pi^{*}, \pi \pi^{*}$ or CT (intramolecular charge transfer band). Spectroscopic data, as well as the reactivity of these compounds, tend to support the general claim by Pitts, Porter, and Hammond in depicting the substituent effect on the reactivity of various benzophenones. In the aromatic ketones studied, a series of compounds differed very little in the energies of the lowest electronic levels, but appeared to differ profoundly in the electronic distribution, and consequently in the reactivity (198).

Because the primary process leading to benzamide is initiated in the excited singlet state of benzaldoxime, inspection of the general features of the ultraviolet absorption spectra is instructive in providing clues as to whether similar arguments, invoked by these workers who explained the substituent effect on the reactivity of benzophenones, could be extended to the benzaldoximes.

Firstly, no $n \pi^{*}$ singlet transition band is observed for the benzaldoximes since oximation removed this transition. 
Secondly, substitution into the aromatic nucleus of the benzaldoximes did not introduce any new absorption band and the general contour of the absorption spectra remains unchanged. Characteristic vibronic structures of the ${ }^{{ }^{1}} I_{b}$ transition band appeared in all spectra and no anomalous features were observed. Since all oximes were excited at $290 \mathrm{~m} \mu$, an obvious change in the spectra of the ${ }^{I_{I_{b}}}$ band upon substitution might have been expected to be a critical factor in accounting for the sustituent effect on the rearrangement. Moreover, the positions of the absorption bands of the oximes are not very sensitive to changes in polarity of the solvents. The insensitivity of the absorption spectra to solvent polarity is more characteristic of $\pi \pi^{*}$ transition than of $n \pi^{*}$ transition band and, in particular, than that of the CT band.

From the foregoing, the observed decrease in the quantum efficiency of the rearrangement is unlikely to be due to modifications of the configuration of the excited singlet state, but rather due to differences in the nature of the excited $\pi \pi^{*}$ singlet states. These are different from the changes in configuration from $n \pi^{*}$ to $\pi \pi^{*}$ which occur. upon excitation of certain substituted benzophenones.

A qualitative description of the substituent effect on the excited $\pi \pi^{*}$ singlet state of the benzaldoxime would be more meaningful if the electronic distribution in the region 
of three main centres, (oxygen, nitrogen and occarbon), involved in the rearrangement, could be adequately defined. Theoretically, this information for two centres, namely, the oxygen and the nitrogen atoms, should be obtainable provided the change in acidity of the exc1ted states represented by processes (43) and (44) (Scheme V) could be calculated. The electronic energies of transistion of $\mathrm{HB}, \mathrm{H}_{2} \mathrm{~B}^{+}$and $\mathrm{B}$ could be experimentally determined, and hence, the change in acidity can be calculated.

Eq. 43

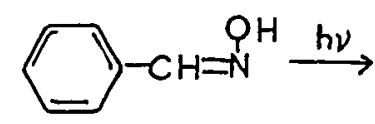

$\mathrm{HB}$

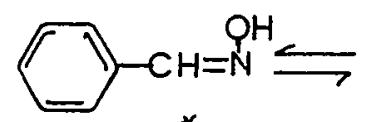

$H B^{*}$

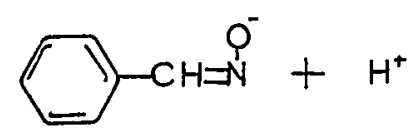

$B^{*}$

Eq. 44

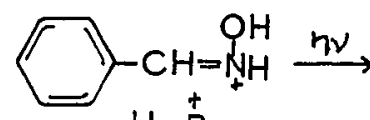

$\mathrm{H}_{2} \stackrel{+}{\mathrm{B}}$<smiles>C=C1NC(C)[B]C1c1ccccc1</smiles>
$\mathrm{H}_{2} \mathrm{~B}^{*}$

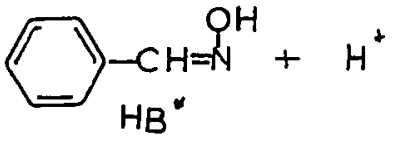

$H B^{*}$

Scheme $V$

In process (43), the oxime is the acld whereas in process (44), It serves as a conjugate base of the protonated form. Any theoretically determined change in the acldity $\left(\triangle_{p^{K}}{ }_{a}^{*}\right)$ between the acidity of the first excited singlet and ground state should, therefore, give some indication of the change to be expected in the electronic distribution on the oxygen and the nitrogen atom.

It is well known that acidic properties of a compound are influenced by the electronic environment at the acidic centres. Any change in the charge density at this site should be reflected by a corresponding change in the acidity. 
In the ground state, the strengths of aromatic acids and bases are known to be a sensitive function of electronic distribution in the aromatic ring. Variations occur over a wide range of $\mathrm{p}_{\mathrm{K}}$ values in the presence of electron withdrawing or electron repelling substituents. Similarly, variation of electronic distribution in a molecule may occur upon excitation. The substituents on the aromatic nucleus then may influence the centres involved in the reaction and drastic reorganization of electronic distribution might be expected in the excited states. For benzaldoxime, the change in the acidity $\left(\mathrm{p}_{\mathrm{s}}{ }^{*}\right)$ of the first excited state needs only to be considered since the rearrangement proceeds from this.

Since the pioneering work of Forster, Weller (200) and Porter (201), a large number of $\mathrm{p}_{\mathrm{a}}{ }^{*}$ values of excited states of numerous compounds have become available. Whereas determinations of the acidity of excited singlets $\left(\mathrm{p}_{\mathrm{s}}{ }^{*}\right)$ have been made from fluorescence data, Jaffe (202) has pointed out that, in favourable cases for which the absorption spectra of both the acid and its conjugate base can be measured, absorption spectroscopic data can be utilized to calculate the change in $\mathrm{p}_{\mathrm{a}}$ values. It has also been pointed out that $\mathrm{p}_{\mathrm{s}}{ }_{\mathrm{s}}^{*}$ can be determined from the positions of corresponding absorption maxima of an acid and its conjugate base. Mason (203) has shown that $\mathrm{p}_{\mathrm{s}}{ }^{*}$ values determined by an absorption method and an emission technique are identical, thus giving assurance of the validity of the 
former. Under favourable conditions, the appltication of the Förster-Cycle, allows one to calculate the change in the $\mathrm{p}^{\mathrm{K}} \mathrm{a}$ values between the excited singlet state and the ground state by the following equation at $25^{\circ} \mathrm{C}$ :

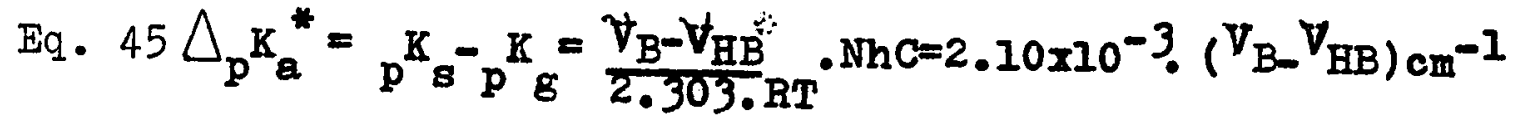

$\nabla_{B}=$ is the frequency, in wavenumbers, of a given transition for the conjugate base and $V_{B H}$ is the frequency of the same transition for the acid. Absorption spectra of benzaldoxime (HB), its conjugate base $(B)$ and its protonated form were determined experimentally and the $\Delta_{\mathrm{p}} \mathrm{K}_{a}$ for processes (43) and (44) calculated as -6.20 and +4.1 units. Use of data obtained in trifluroacetic acid in cyclohexane solution gave $\Delta_{p^{K}}{ }_{a}^{*}=+4.9$. These changes in the $p_{a}$ values upon excitation are in good agreement with the analogous systems. For the system of 2,4-dinitrophenyl hydrazone of benzaldehyde 114, represented by Reaction 30 :

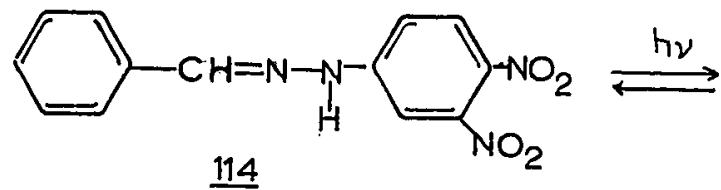

$\underline{114}$

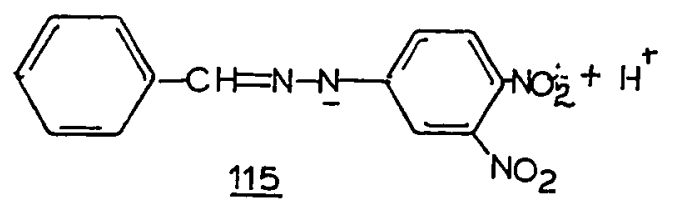

115

Reaction 30

$\triangle_{\mathrm{p}} \mathrm{K}_{\mathrm{a}}^{*}$ is as large as (10.0) $\mathrm{p}_{\mathrm{a}}^{\mathrm{K}}$ units. The change was from 10.9 in ground state to 0.89 in the excited singlet state (202). For process (44) Weller (200) reported that $\mathrm{p}_{\mathrm{s}}^{*}$ of anil systems $(116)$ is always greater than $p_{g^{*}}^{* *} \triangle_{p} K_{a}^{*}$ is always positive. 


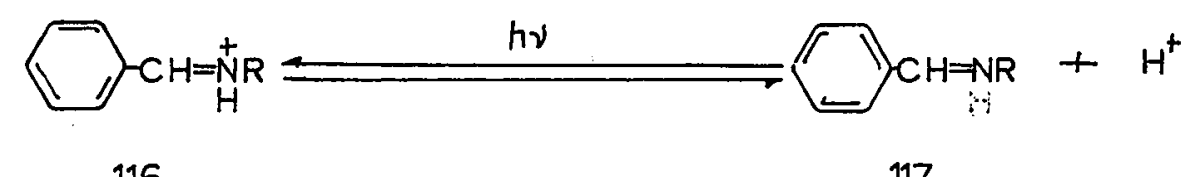

116

$\underline{117}$

Reaction 31

Therefore, the trend in acidity change is, indeed, in the right direction for benzaldoxime.

The spectrum of the conjugate base (B) of benzaldoxime was measured in concentrated alkali and a bathochromic shift from $252 m \mu$ to $272 m \mu$ observed. (See Fig. 18). This shift of $20 \mathrm{~m} \mu$ is equivalent to a change in frequency of $\Delta V=-2950 \mathrm{~cm}^{-1}$. A similar bathochromic shift from 252 to $265 \mathrm{~m} \mu$ for the protonated species was observed and this shift is equivalent to $\Delta \nu=+2000 \mathrm{~cm}^{-1}$. In cyclohexane solution containing trifluoroacetic acid, the shift is $+15 \mathrm{~m} \mu$. This shift of +13 to $15 \mathrm{~m} \mu$ is comparable to a shift of $+14 \mathrm{~m} \mu$ observed for benzilidene methyl amine (178). In both instances, spectra of the deprotonated and protonated species of benzaldoxime were taken immediately after preparation. In this way, hydrolysis from benzaldoxime to benzaldehyde was prevented. After two hours, the $\lambda_{\max }$ observed was that of benzaldehyde. Surprisingly, the conjugate base in the alkaline solution is stable even after two hours. The absence of any new bands between $230 \mathrm{~m} \mu-$ $250 \mathrm{~m} \mu$ in an alkaline solution indicated that benzonitrile $(223 \mathrm{~m} \mu, \epsilon=13,400) \%,(272 \mathrm{~m} \mu, \epsilon=730)$, benzaldehyde (248m $\mu$, $\epsilon=12,500,272 \mathrm{~m} \mu, \epsilon=1520)$, and benzoic acid $(222 \mathrm{~m} \mu, \epsilon=9180$, 
$272 m \mu, \epsilon=874$ ) were not interfering with the spectra of the deprotonated form of benzaldoxime. From the known value of $\mathrm{p}_{\mathrm{K}}^{\mathrm{K}} \mathrm{g},(204)^{*}$ for process (43), Scheme $\mathrm{V}, \mathrm{p}_{\mathrm{s}}^{\mathrm{K}}$ (of the oxime in its first excited state was calculated to be $+4.5 \cdot{ } k_{s}^{*}$ of the protonated form in its excited singlet state is calculated as +3.1 in aqueous solution and +3.9 in cyclohexane, using the value $\mathrm{p}_{\mathrm{g}}=-1$ for methyl ethyl ketoxime (204). A value for an aliphatic oxime is not the most appropriate choice, but was used since no value for benzaldoxime is available.

Consideration of the possible existence of processes 43 and 44 (Scheme V) leads to the conclusion that the electron density is probably decreased at oxygen and slightly increased at nitrogen in the excited singlet state of benzaldoxime. Owing to the proximity of these centres, the corresponding decrease at one centre might be exerted on the adjacent atom via an oxygen-nitrogen bond. As for the $\alpha$-carbon, the change in the electron density might be ascertained by considering the following. Theoretical Simple Molecular Orbital (SMO) calculations for benzilidene methyl amine, the UV spectrum of which is virtually superimposed: : on those of styrene and benzaldoxime, showed only a slight increase at the nitrogen atom upon excitation to the first excited singlet level (6.24\%), but a larger increase at the $\alpha$-carbon (29.5\%). In benzaldoxime, the accumulation of the electron density on the $\alpha$-carbon might also be expected since transitions of the first excited states appear to be similar to those of 
benzilidene methyl amine. The enhanced electron density at the $\alpha$-carbon could influence the migration of the oxygen atom with its nonbonded Ione-pair.

Based on the foregoing, any one of the three centres directly involved in the rearrangement might be influenced by substituents, solvents, and acids. In the excited singlet state, the course of reaction then could be affected by the direct resonance interaction of substituents with the aromatic ring and the oxime moiety. This interaction results in an enhanced electron density of the $\alpha$-carbon upon excitation to the singlet. An increase at the $\alpha$-carbon might be expected to prevent facile migration of the oxygen atom with its electron pair from the nitrogen to the $q$-carbon atom. Contributions of $118 \mathrm{~b}$ and $118 \mathrm{~d}$ to the resonance structures 118 might then be important.

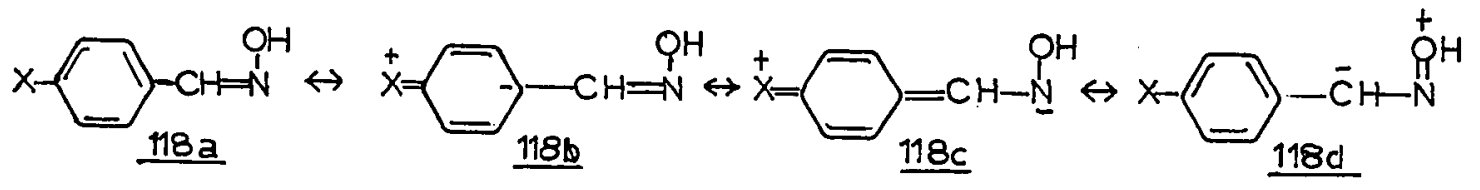

The influence of the resonance effect on the quantum yield is guggested by the parallelism between the trend of the ultraviolet spectra and the photolytic yield, in the sense that a large resonance effect would explain both the bathochromic shift and the increased intensity of absorption of the $\left({ }^{I} I_{b}\right.$ and $\left.{ }^{I_{I_{a}}}\right)$ bands. Such behaviour is accompanied by a decrease in the quantum yield of benzamides. For p-substituted benzaldoximes, the bathochromic shift was found to decrease in 
the order $-\mathrm{MeO}>\mathrm{Br}>\mathrm{CI}>\mathrm{Me}>\mathrm{F}>\mathrm{H}$ as that reported for mono-subst1tuted benzenes (ref. 154, p.247). For benzene such a shlft has been malnly due to a direct resonapce participation of the lone pair of electron. The Importance of resonance interaction in the excited state is consistent with the results obtained for benzaldoxime and p-fluorobenzaldoxime in that, not only are the spectra of these two oximes almost 1dentical, but so also are the quantum yields for the rearrangement, 0.025 and 0.021 , respectively. Both can be accounted for by the reduced importance of resonance interaction of the fluorine atom with the aromatic nucleus in the excited state (205). The energy separation between the 0-0 singlet band of these two compounds is not greater than $0.5 \mathrm{k} . \mathrm{cal}$.

Recently, it has been reported that a positive correlation with the chemical shift $(\delta)$ of $\alpha$-proton of p-substituted benzaldoximes with the Hammett sigma parameter $(\sigma)$ indicates that the electron density is decreased by electron-withdrawing groups located para to the oxime molety (206). In vien of the recent report of Jaffe (202) and Wehry (205), that the acidity functions of the excited states of phenols could also be correlated with the Hammett's sigma function, the efficlency of the photochemical conversion might be expected to follow a definite trend, Increasing upon substitution with groups having higher sigma values. The $\alpha$-carbon of the oxime might also be expected to be influenced by electron-withdrafing groups in the excited singlet as well as the ground 
state. The results certainly cannotbinterpret by invoking such a point of view. If, however, such an explanation is justified, then a competing effect must begin to dominate the reaction. Such an effective competition might be facilitated by removal of the singlet to the ground state, or by enhanced singlet-triplet(S-T) (ref. 14 p.302) intersystem crossing due to a "heavy atom effect". In benzaldoxime, radiationless transition from the excited singlet to the ground state was previously suggested on the basis of the brief study of cistrans isomerization.

Despite the voluminous photochemical literature, there has been little systematic study of substituent and solvent effects on these reactions. Attempts have been made to correlate the efficiency of the reactions with the Hammett sigma parameters $(206,207)$. Where correlations are found, negative, rho values have been obtained. The most extensive investigation concerns the mechanism of the primary process of the photochemical Wolff rearrangement of p-substituted diazoacetophenones represented by the following reaction:

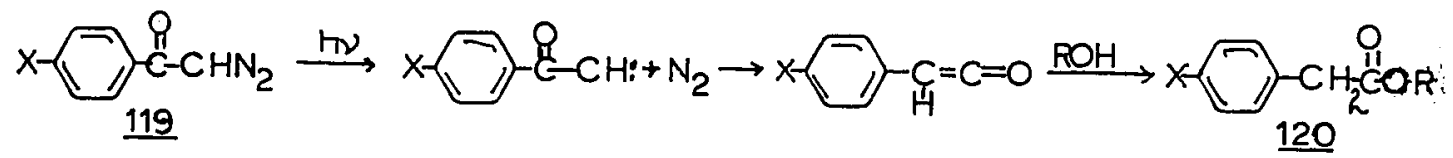

\section{Reaction 32}

In correlating the quantum yield of the reaction, Ziffer had accepted the negative rho value because of the probable intervention of a triplet state. Recent work of Padwa (208), 
however, has conclusively established that the rearrangement proceeds via a singlet rather than a triplet. It was also observed that the rate of nitrogen elimination appeared to be lowered by substitution (209). In methanol, this trend. was observed by Kimse and Horner (210) and has been verifled by Mazzucato (209). A general lowering of the gleld by polar substituents was also notlceable, but there is no correlation of reactivity with the lonizing power of the solvents. To eccount for these results, the 1mportance of the resonence interaction in the excited molecules has been stressed. But the nature of the electronic interaction between the substituents and the solvents and the excited states st1ll remains to be defined. Of interest here is the similarity of the photochemical Beckmann and the Wolff rearrangements, both of which are inltiated in the excited. singlet state, in their response to solvent and substituent effects.

\section{Effects of Acids and Solvents on the Bxcited Singlet} State of Benzaldoximes

When f1rst reported, it was not clear whether the over-all photochemical reaction of benzaldorimes was acid catalyzed, or whether the rates were a function of the lonizing power* (109)

\footnotetext{
* The most comprehensive correlation with the lonizing power has been determined by Dimroth, Relchardt, Slepman, and Bohlman (109). Energies of transition ( $E_{\mathrm{T}}$ of ptridinium$\mathrm{N}$-phenyl betaine) in varlous solvents were used as a measure of the lonizing porrer of the solvents). $E_{T}$ here is to be distingulshed from the triplet energy $E_{\mathrm{T}}$.
} 
of the solvents. The results for 1rradiations conducted at $290 \mathrm{mp}$ are tabulated in Tables III, XI, XII and Illustrated In F1g. 16, 17, and 18. These data establish conclusively that protonic solvents, especially aclds, are necessary for an efficient photochemical transformation. In aprotic solvents (acetonitrile, dioxane, and cyclohexane) without ac1ds, the quantum efficiency is low $(0.002$ to 0.004$)$, but, upon the addition of acetic acid the ylelds increase to 0.007 to 0.025 . Without aclds in protonlc solvents such as methanol, ethanol and $80 \%$ ethanol-water, the quantum ylelds Iie between 0.009 and 0.013 . The maximum quantum jield, 0.058 , obtained in neat acetic ac1d. It was also demonstrated that acid 18 necessary for the rearrangement of p-methoxyacetophenone oxime to the corresponding amides. The quantum yields of the amides, under aprotic and protonic conditions, differ by a factor of 13, comparable with that of the rearrangement of p-anisaldoxime under the same experimental conditions.

The results from the investigation of the solvent effect served to demonstrate that the quantum jield of the over-all reaction could not be correlated with the lonizing power of the solvents (see F1g. 16). With the standard concentration of acetic acid (0.73M) in non-protonic solvents, the highest yield was recorded in cyclohexane and the lowest in dioxane. No over-all trend was observed for these solvents. Moreover, 
In protonic solvents, the quantum efficiency differed by a factor of 18 between acet1c ac1d and ethanol, although the lonlzing powers of these two solvents differ by only $0.1 \mathrm{k} . c a l$. Even more surprising is the difference between the quantum rields in methenol and ethanol. In the former solvent, the yield is as high as 0.011 , but in ethanol, the quantum jield is only 0.003, comparable with the value obtained in aprotic solvents. Because of the low quantum field in ethanol, its value was redetermined several times. One would not have expected to observe such a large difference because of the similar nature of these two solvents. The over-all reaction is considerably more complicated than expected and the small difference between the solvents themselves must be a critical factor. Further complications appeared to be introduced by the difference in the nature of the aclds too. In F1g. 17, the plot of the reciprocal of the quantum yield $\left(\frac{1}{\Phi_{B_{2}}}\right.$ ) against the reciprocal of the concentration of acetic acid and trifluoroacetic acid (A) $\left(1 / \mathrm{A} \mathrm{IM}^{-1}\right)$ is shown. It can be seen that the change in the quantum yleld of the rearrangement for trifluoroacetic acid is less sensitive to the change in the concentration than for acetic ac1d over the ranges 0.007 to $0.071 \mathrm{M}$. The change in the efficiency of the rearrangement for acetic acid is more pronounced. Moreover, although the addition of both acetic acid and trifluoroacetic acid to the solution of cyclohexane increases both the rate and the quantum jield of benzamide, the difference is less than might have been anticipated on the 
basis of the relative acid strengths (about $5 \mathrm{p}^{\mathrm{K}_{2}}$ units (2II). When irradiated in the presence of the same concentration of acetic acid and trifluoroacetic acid under identical conditions, benzaldoxime afforded benzamide with greater efficiency for the latter by a factor of only 5 .

A : posstble. explanation of the different results with the two acids is provided by the reaction of benzilidene methyl amine with hydrochloric acid since benzaldoxime and the anil behave similarily toward acids. In concentrated sulfuric acid (about $3 \mathrm{MH}$ ) the spectra of both protonated forms undergo a large bathochromic shift by 13-14m (see Fig. 18: for spectrum of $\alpha$-benzaldoxime under various conditions). In this solution, on standing, the final hydrolysis product is benzaldehyde. Also, not only were their protonated forms observed in about the same region of the ultraviolet spectra, but they undergo the same type of electronic transition. Kubota (178b) reported that in ethanol, the concentration of hydrochloride required in solution to convert the anil to its protonated form, is about $1 \times 10^{-3}$ M. in the presence of a spectroscopic amount of benzilidene methyl amine. Since trifluoroacetic acid and hydrochloric acid are of comparable acid strength, one might also expect to find benzaldoxime protonated to about the same extent by trifluoroacetic acid as benzilidene methyl amine is protonated in the presence of hydrochloride in ethanol. In fact, the experiment bears out this expectation. The maximum at $250 \mathrm{~m} \mu$ in the vltraviolet 
spectrum of benzaldoxime in cyclohexape exhibits a "red" shift of $+15 \mathrm{mp}$ in the presence of trifluoroacetic acid. This clearly demonstrates that protonation of the oxime occurs to a significant extent. Unlike the spectrum of benzaldoxime In neat acetic acid, which exh1bited vibronic structures of the ${ }^{I_{L_{b}}}$ and, the spectrum taken in the presence of trifluoroacetic acid does not display any of the vibronic structure of this electronic band. Vibronic structure of the 0-0 singlet $I_{I_{b}}$ band appeared at $290.5 \mathrm{mp}$ in cyclohexane, at $292 \mathrm{mp}$ in water and at $294 \mathrm{mp}$ in acetic acid. The retention of the vibronic structures in the presence of acetic acid indicates that excitation of benzaldoxime is essentially due to the neutral oxime 1tself, rather than to the protonated form. In the presence of a stronger acid, trifluoroacetic acid, the absorption of light by benzaldoxime however, must be largely due to the protonated form $\left(\mathrm{H}_{2} \mathrm{~B}^{+}\right)$. The difference in the results for acetic acid and trifluoroacetic acid therefore does appear to be due to the difference in the nature of acids. In the presence of trifluoroacetic acid, one step (protonation of the oxime) may have been by-passed by direct excitation of benzaldoxime $\left(\mathrm{H}_{2} \mathrm{~B}^{+}\right)$. The quantum $y$ ield of the rearrangement might then be expected to be independent of the acid concentration. The radiant energy absorbed by the protonated. form could then be essentlally utilized for the migration of the oxygen atom. In the presence of a weak acid (acetic acid) however, an appreciable amount of the radiant energy of the electronic state may be expended on the removal of the 
tightly bonded proton on the $0-H$ group of the acid; hence a greater dependence on the concentration of the acid may be expected. This explanation of the observed difference in the behaviour of the reactions of two acids is plausible, provided that the involvement of the acid in the excited singlet state overwhelmingly determines the observed results.

From the observed results (Fig. 16) it appears that participation of the solvents could be important in the reaction. Considering that $\pi \pi^{*}$ excitations are generally believed to be more highly polarized in the excited states than the ground state $(182,212)$, and in view of the possible drastic electronic re-organization of the excited benzaldoxime molecule at the three reactive sites involved in the migration of the oxygen atom, the influence of the solvents on the rearrangement of the oxime in the excited state cannot be entirely ignored.

The application of the Förster-Cycle to the calculation of the change in the pra value of the excited state of benzaldoxime led one to infer that an increase in the electron density at the nitrogen atom and a decrease in the electron density at the oxygen could occur. Therefore, it would be expected that the nitrogen would be more susceptible to protonation or solvation by electron defficient centres of the solvents and that the region around the $\mathrm{O}-\mathrm{H}$ bond of the oxime would be influenced by basic, or electron-rich centres of the solvent molecules. Enhanced interaction of the type 
I2I and type 122 in the excited $\pi \pi$ singlet state would then be greater than in the ground state $\underline{121}$ and $\underline{122}$ where $X$ represents a basic centre of a solvent molecule.

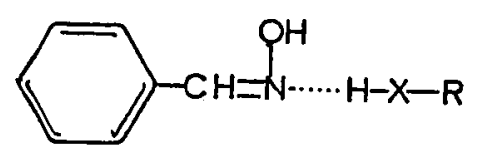

121

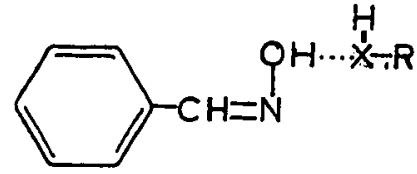

122

The observed solvent effects might then reflect the relative importance of the types of interaction of 121 or 122 in the excited state. In an analogous system such as benzaldoxime, the primary formation of the oxazirane from the irradiation of nitrones is found to be highly dependent on hydrogen bonding between the solvent molecules and the oxygen atom of the nitrone moiety. In ethanol, the quantum yield for the formation of the oxazirane from $a-N$-diphenyl nitrone was reduced from 0.28 to 0.18 , compared with the efficiency in cyclohexane $(68,69)$. In benzaldoxime, a similar effect of the polar solvents in the region of the $0-H$ bond might also be expected to occur. The initial formation of the oxazirane from nitrones and benzaldoximes, however, would be expected to be somewhat different. The nitrone does not require protonation, whereas the oxime does. For efficient formation of the oxazirane from the oxime, the migration of the oxygen and protonation of the nitrogen might demand concomitant participation. In aprotic solvents and in the absence of external acids, a proton can be supplied to the nitrogen of 
the excited species from the unexcited oxime itself. This abstraction of hydrogen would not be expected to be facile and a poor yield would therefore be expected. Enhanced self association might also be expected to occur upon excitation. In fact, the yield was very poor in the aprotic solvents. By introducing a proton source (acetic acid or trifluoroacetic acid) the extent of protonation on the nitrogen would be similar in each of these solvents. Protonation would be relatively fast. Since the extent of the protonation is similar, the difference in the quantum yield presumably reflects the participation of a solvent molecule on oxygen migration in the excited singlet state due to enhanced association of the type 122. Thus, a basic solvent is capable of retarding the oxygen migration. Of the three aprotic solvents, cyclohexane is neutral, and dioxane is more basic than acetonitrile (204). Therefore, if the difference in the efficiency is a result of interaction of type 122, then the order of the yields expected would be cyclohexane > acetonitrile>dioxane. The observed respective values, $0.025,0.014$, and 0.004 , are consistent with the argument. The differences between the various solvents might be governed by the relative importance of their interactions with oxime molecules in the excited state. In comparing the difference between the yields in aprotic and protonic solvents, and the difference between the yields in acetic acid and alcohol, the larger quantum efficiencies in acetic acid and protonic solvents (vs aprotic solvents) 
could be due to the appreciable significance of the nitrogen atom protonation rather than to the hindrance of the oxygen atom mitration. The disparity between the yields obtained in methanol and ethanol is anomalous. The large difference in the yields however, might also be explained in a similar manner, where the basicity of the ethanol is of greater importance than its acidity compared to methanol. A pKa value of ethanol has been reported as high as 18 (213a) and 19 (213a) and of methanol, about 16 (ref. 204, p.223). Crandall (212) has suggested, from studies of solvent effects on the ultraviolet spectra, that primary alcohols, except for methanol, act predominanty as proton acceptor rather than as donor. Comparisons of the relative basicities of ethanol and methanol suggests that ethanol is the more basic (204, p.223,245).

III Effects of Substituents, Solvents, and Acids on the Electronic Ground State of the Intermediate

The difficulties in interpreting the photochemical transformation of benzaldoxime to benzamide, via the 3-phenyloxazirane under warious reaction conditions, are compounded by the fact that at least five different reactions are known for 3-aryloxaziranes in the electronic ground state. These are 


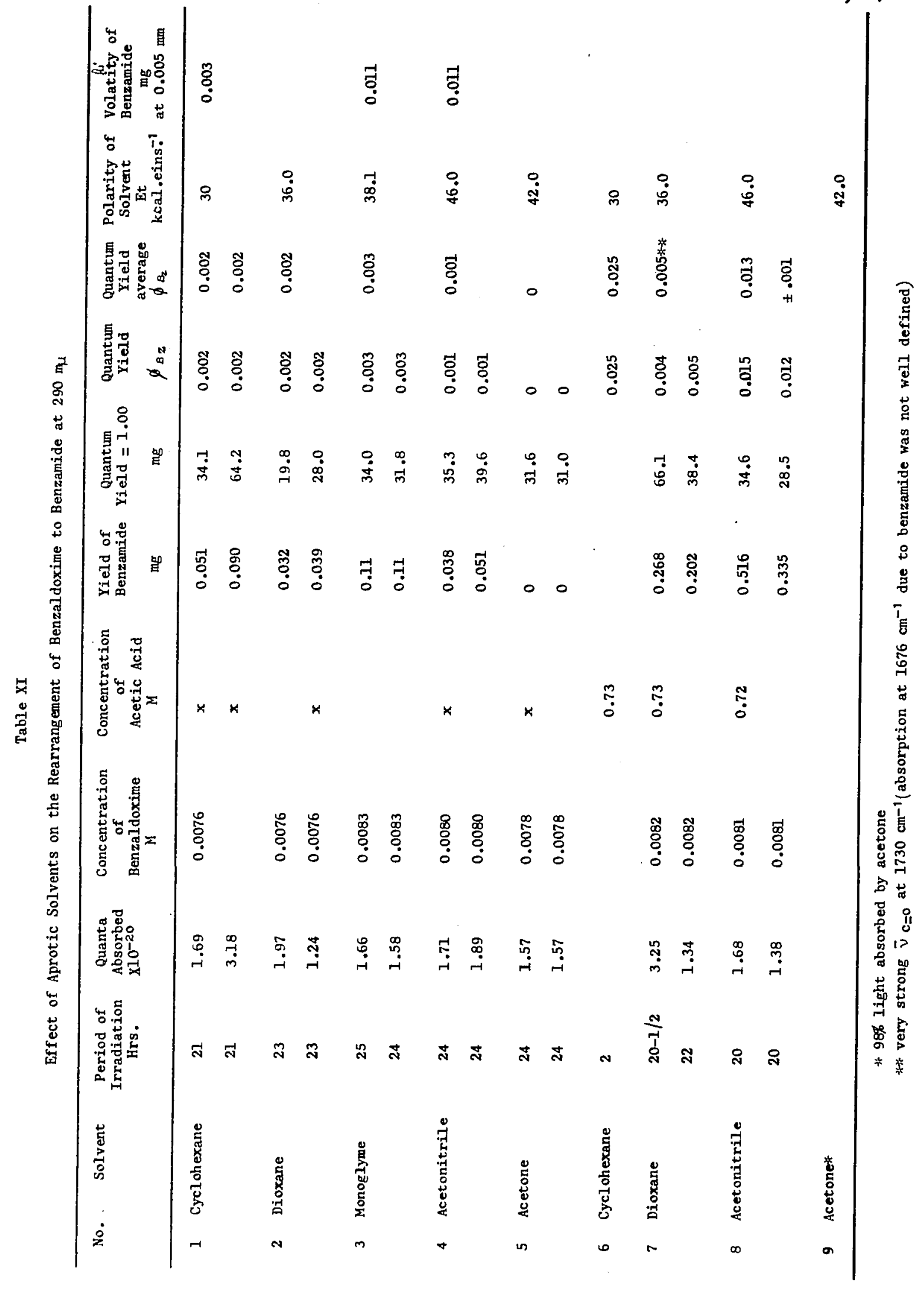




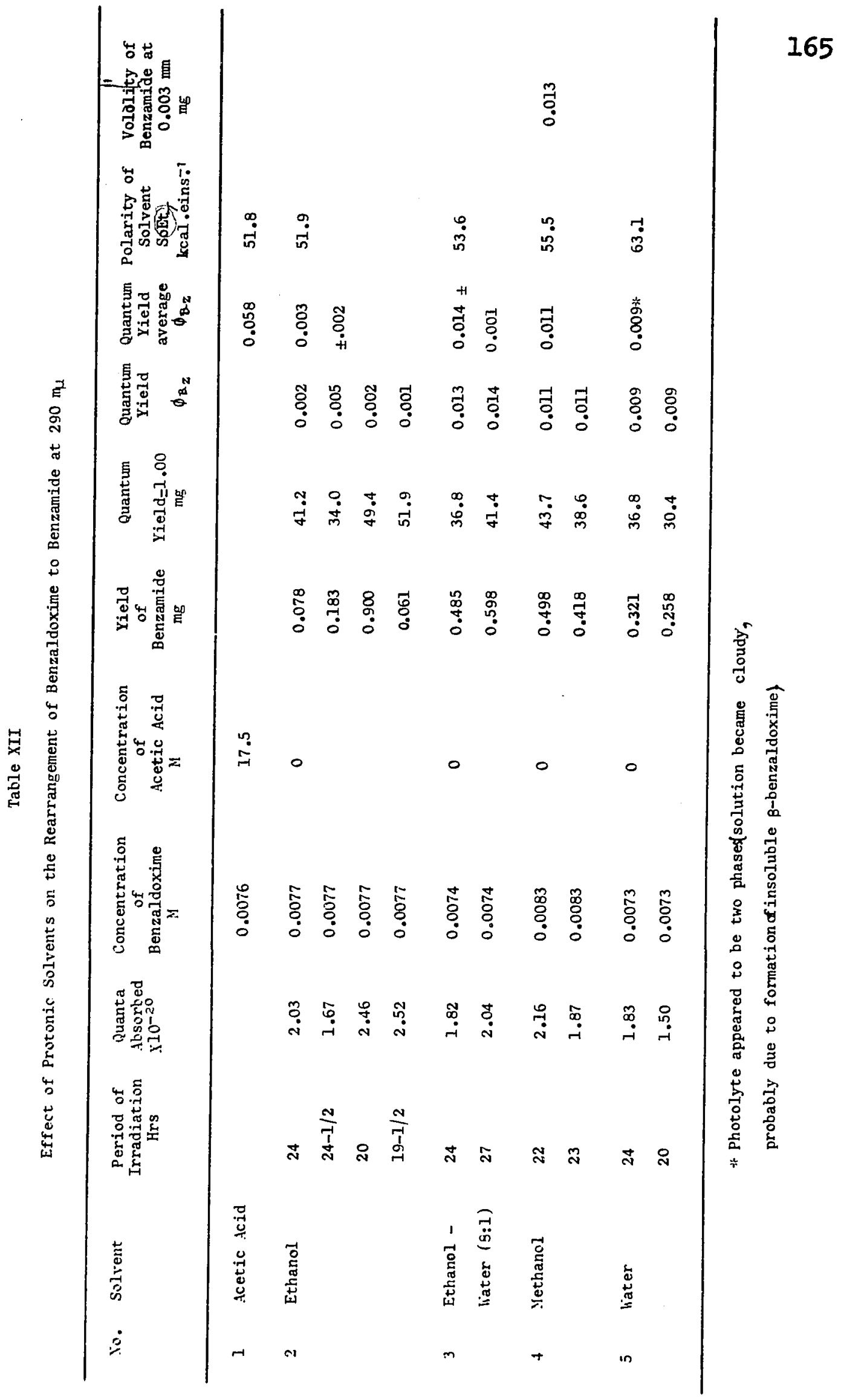




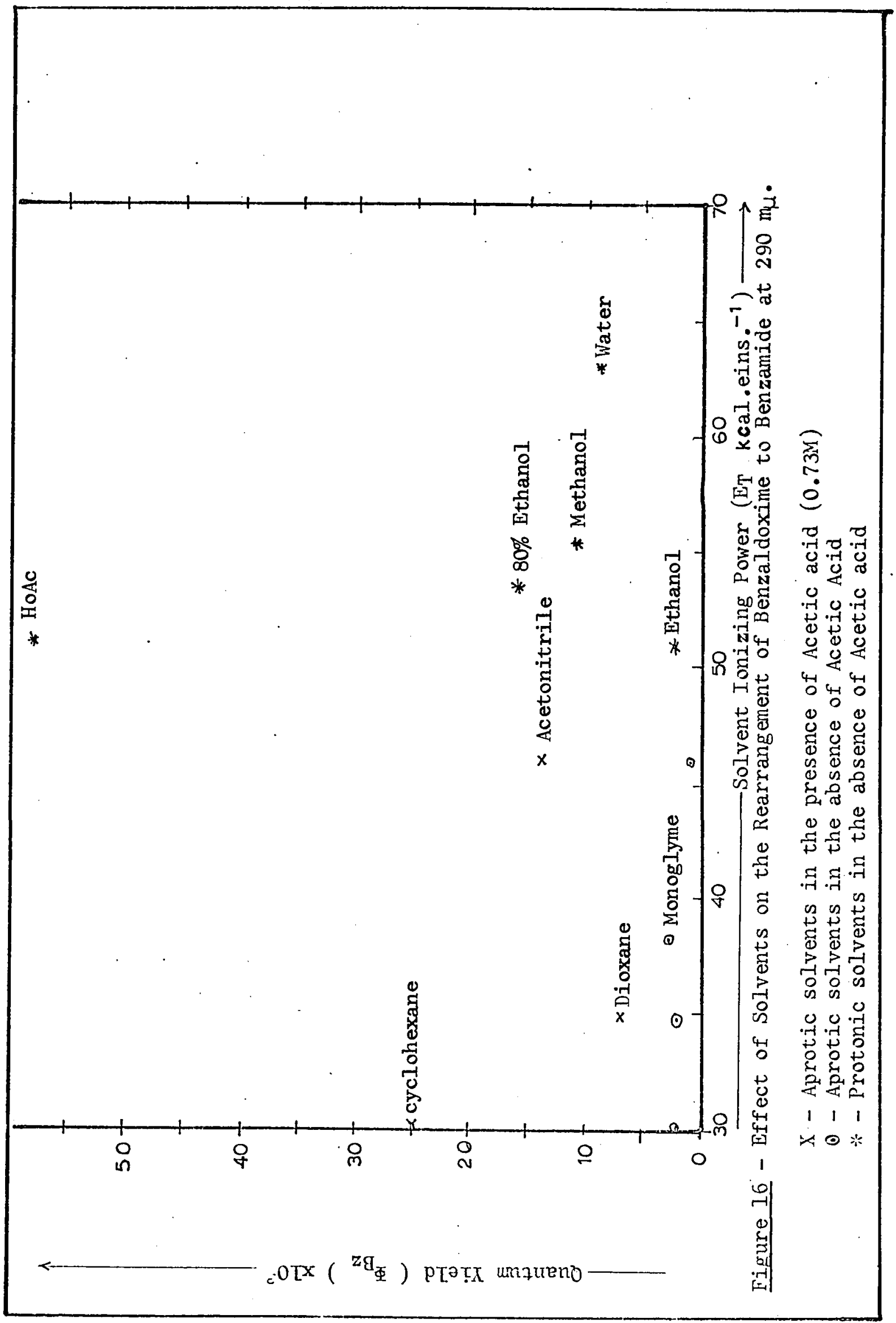




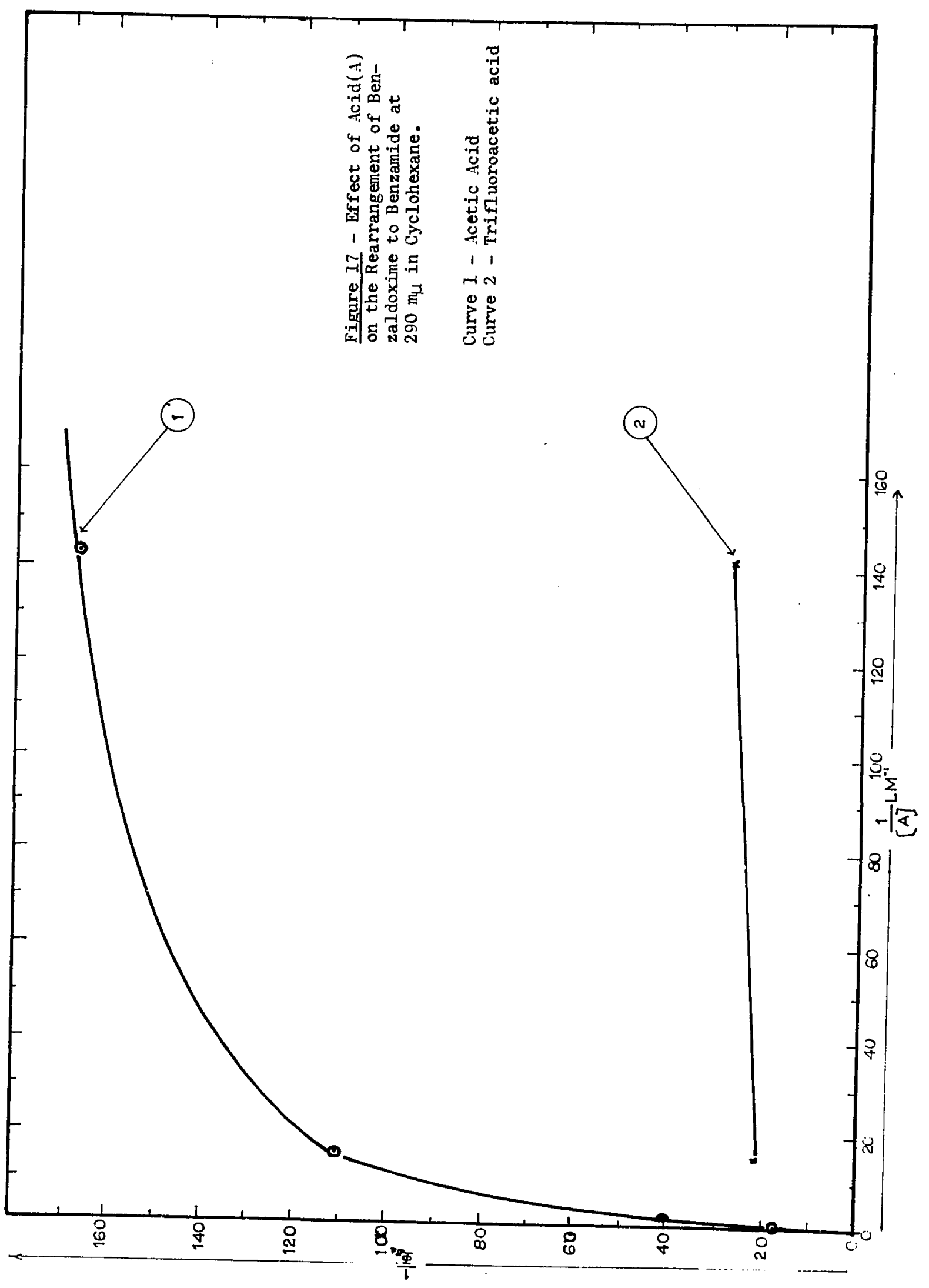




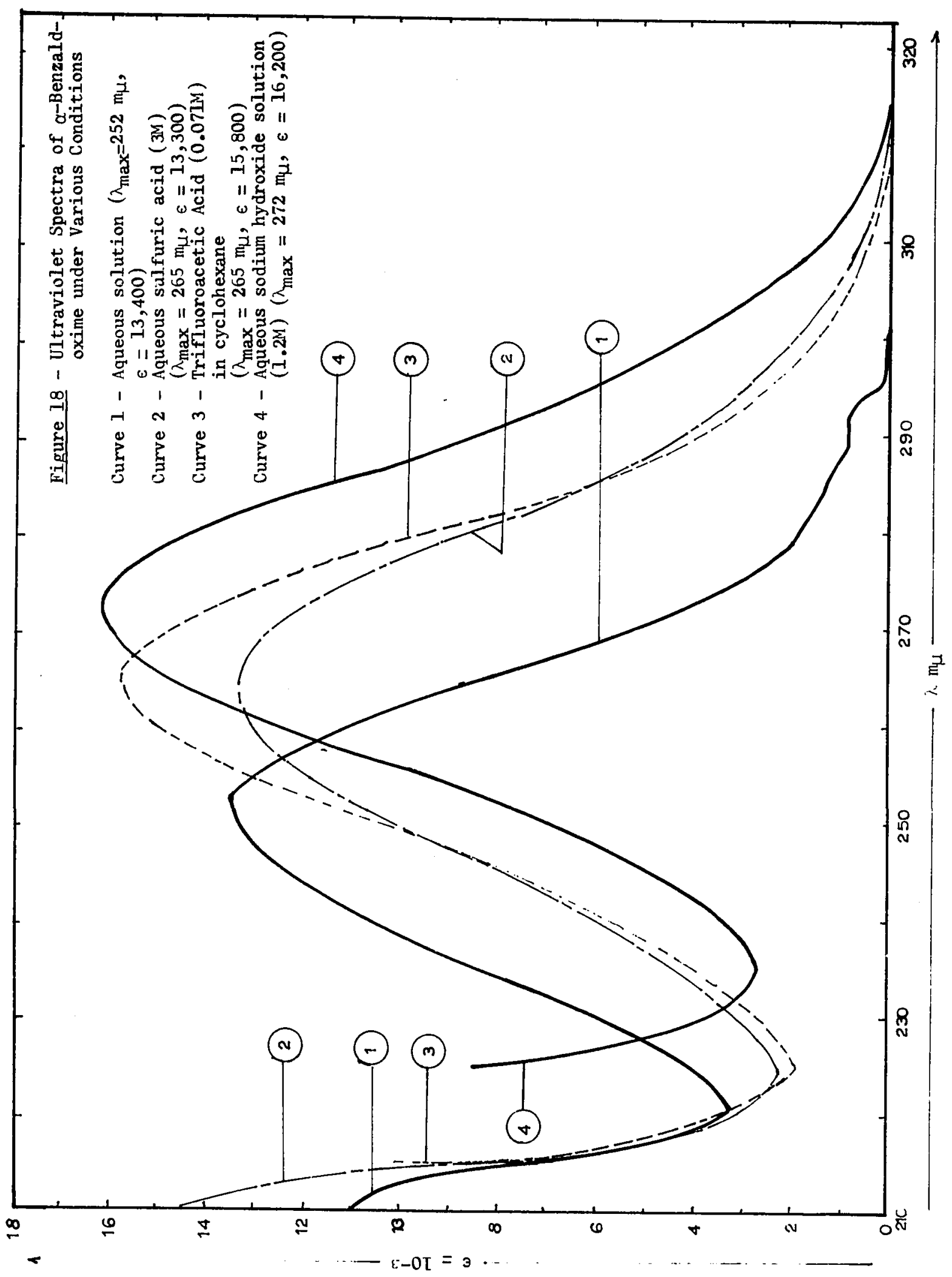


given below $(67 \mathrm{~b}, \mathrm{c})$ :

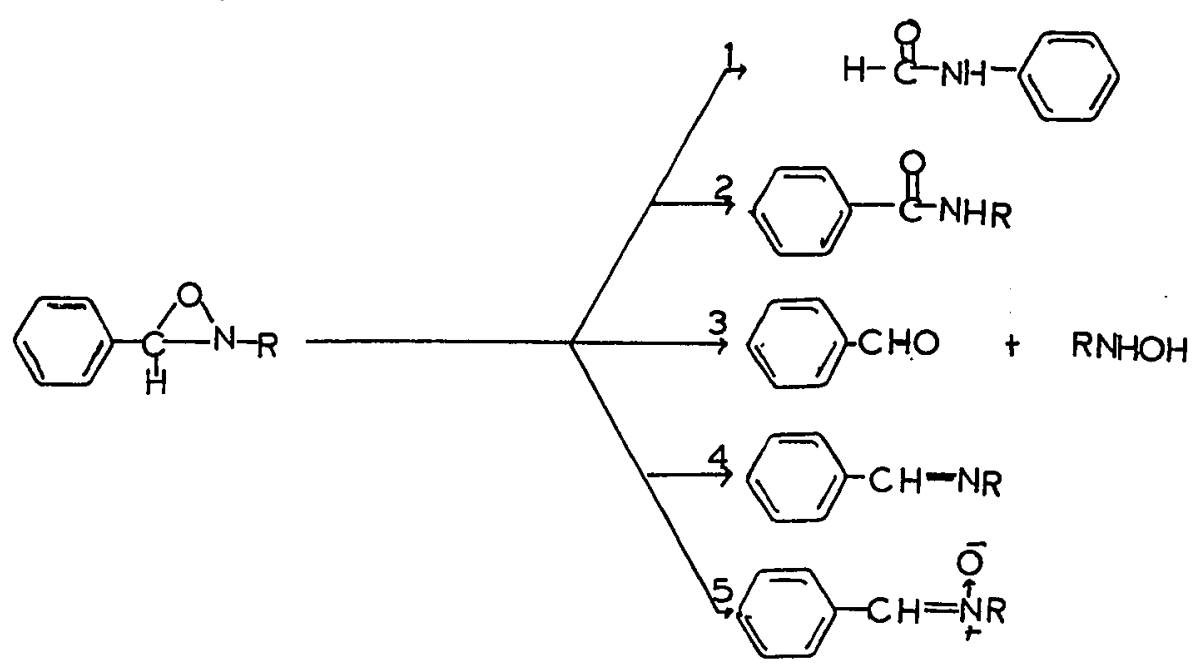

Scheme VI

Little is known about the chemlcal propertles of many oxaziranes because of their thermal instability. Those lacking a N-substituent are particularly labile. Since 3-phenglorazirane 1s of the latter type, rationalization of the effects of subst1tuents, solvents, and ac1ds on 1ts rearrangement 18 difficult $(110 b, c)$. From the observed results, however, plausible mechanlsms of 1 ts rearrangement can be considered. In the electronic ground itate, the rearrangement of 3-phenyloxazirane might be expected to occur in one a several ways. Various possibilities are depicted below:

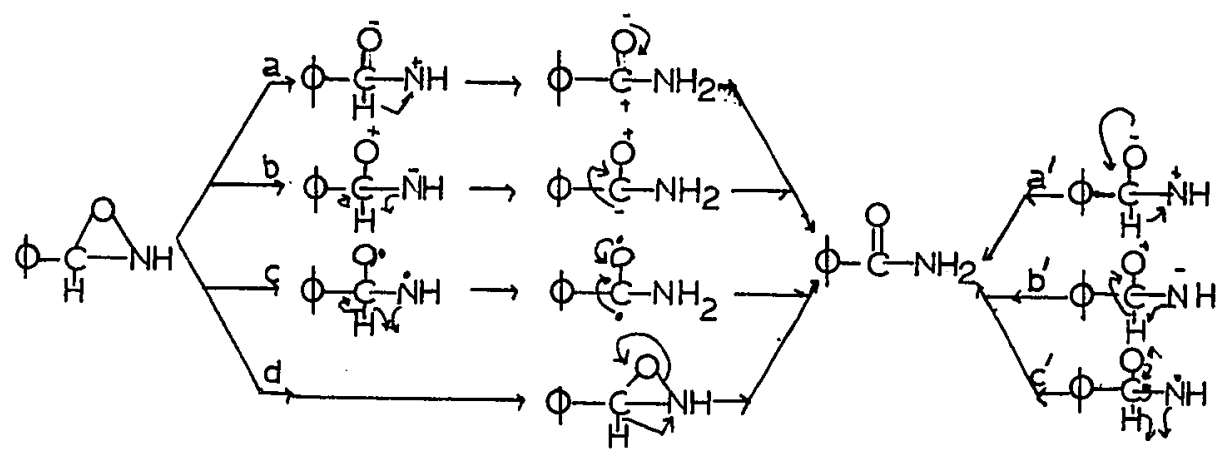


In the presence of acid, a C-O cleavage of 3-phenyl-2t-butyloxazirane has been shown to afford hydrolytic products almost quantitatively. A heterolytic C-O cleavage, initiated by ring protonation, has been suggested to account for the results and is given below:

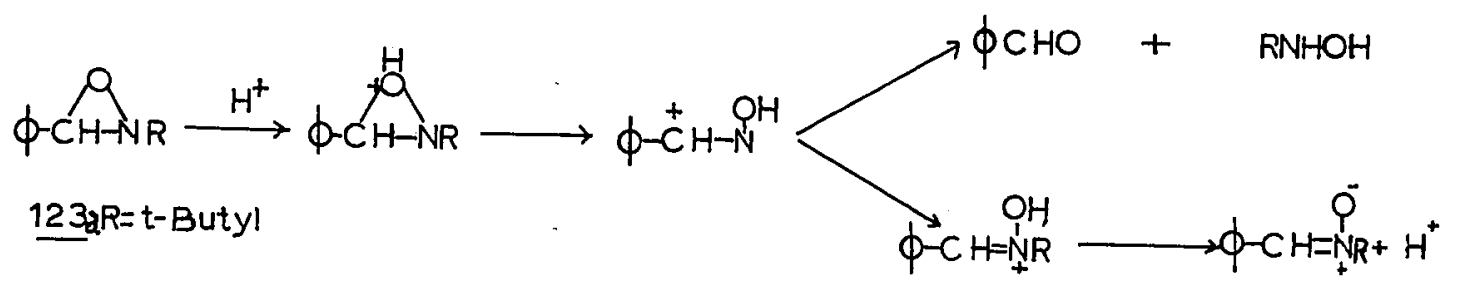

Reaction 33

Emmons has suggested that a nitrone is also involved and is formed by acid catalyzed ring fission at the $\mathrm{C}-\mathrm{O}$ bond. In the present instance, if the hydrolytic products and nonphotochemical return to the starting material were to result from a C-O cleavage, one would expect that a greater amount of reaction would occur in the presence of trifluoroacetic acid then in acetic acid. This would lower the over-all quantum yield of benzamide. (See Reaction 34).

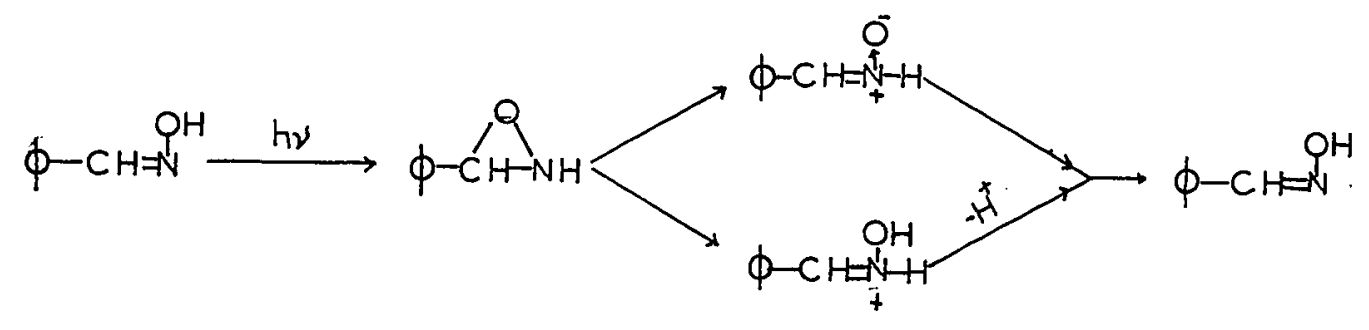

Reaction 34 
In fact, the over-all quantum yield is higher in trifluoroacetic acid. Even in $80 \%$ ethanol, only benzamide is formed and the yield is greater than in methanol. Had there been a large difference between the rates of return or hydrolysis in the presence of water and that of methanol, one might have expected a low quantum efficiency. Under the experimental conditions, no hydrolysis of benzamide to benzoic could be detected. The possibility of non-photochemical return from oxazirane to the oximes cannot be entirely ignored in view of the report of thermal rearrangement of some oxaziranes to compounds containing an oxime moiety. An example is the phenyl isocyanate derivative of 3-ohenyloxazirane (110b), which rearranges thermally in aprotic solvents to the urethan of benzaldoxime (see Reaction 27).

Splitter and Calvin have pointed out from their studies of the rearrangement of 2,3-diphenyloxaziranes, that the formation of amides involving hydrogen migration is less sensitive to a solvent than phenyl migration and, as an explanation, a "no mechanism" type of reaction (215) has been suggested. Additional evidence from studies of oxaziranes partially supports this claim. In a few instances, oxaziranes have been reported to undergo facile migration unaffected by solvents, as exemplified by the rearrangement of 2-cyclohexyl3-phenyl-3- benzoyloxazirane to $\mathrm{N}, \mathrm{N}$-dibenzoylaniline wi thout the formation of nitrone (114b). The 2-phenyl analogue in this particular series is too unstable to be isolated, but 
was presumably formed via an oxazirane. Very recently, Taylor and Spencer (216) reported that irradiation at $254 \mathrm{~m} \mu$ converted 6-methyl phenanthridine-5-oxide 133: in both ethanol and methylene chloride, to the corresponding 5-methyl6(5H)-phenanthridine with a comparable quantum yield of 0.11 to 0.12. An oxazirane, which is too unstable for detection under the experimental conditions, was presumed to be the intermediate.

A heterolytic cleavage represented by pathways* $a$ and a would be expected to lead to the development of negative charge on oxygen. Alternatively, fission by process $b$ or $b^{\prime}$ would be expected to give the opposite charge distribution on the oxygen and the nitrogen atoms. Mechanisms a and $a$, would be favoured over $b$ and $b^{\prime}$ if the rearrangement proceeded by a heterolytic disruption of the ring from the 3-phenyloxazirane to the benzamide for a development of a negative charge on the oxygen atom and a positive charge on the nitrogen. This situation would be expected to be energetically more favourable for intermediates or transition states involved in the rearrangement, than would be a situation in which the charge distributions were reversed. Invariably, where reactions are known to occur by 1,2-carbonium ion shifts, the migratory aptitude is dominated by phenyl migration rather than hydrogen or alkyl migration (217). In the present case, where the intermediacy of 3-phenyloxazirane may be involved, exclusive hydrogen migration is observed. This suggests that

* Reaction pathways denotes by a'b'c' are envisaged as concerted processes whereas steps denoted by $a, b, c$, are
represented as step-wise reactions. 
for most of these 3-phenyloxaziranes, the pathway from the intermediate to the benzamides does not traverse the course a or $a^{\prime}$. In the absence of any phenyl migration (excepting p-anisaldoxime), one must conclude that a heterolytic cleavage via processes $a, a^{\prime}, b$, and $b^{\prime}$ is improbable mode of rearrangement for the intermediate. A further point which provides a basis for the exclusion of $a$ and a' is that, in the presence of acid, the protonated form of the intermediate might be expected to be formed, rather than a charge separated species. In the event of formation of an 0-protonated species, one would expect to find a preponderance of phenyl migration over hydrogen. Of course, the opposite was observed experimentally. Further grounds on which to exclude transformation of 3-phenyloxazirane via pathways a and a' are supported by the behaviour of some oxaziranes in aqueous acidic solution. Emmons (218) has reported that aliphatic oxaziranes, in which a group is present $\alpha$ to the nitrogen atom, decompose to amides and carbonyl compounds. Sequence of the steps accounting for the observations is as follows;

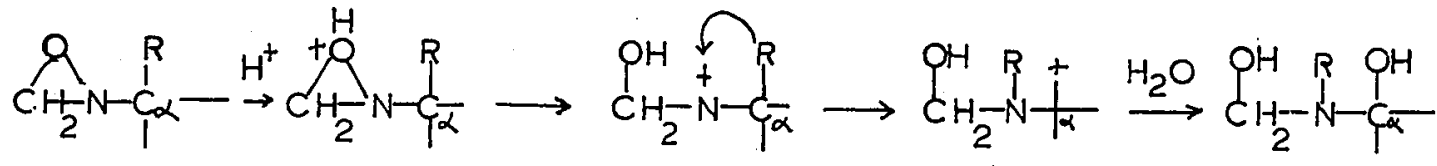

\section{Reaction 35}

In this instance, it has been suggested that the oxygennitrogen $(\mathrm{N}-\mathrm{O})$ bond of the protonated oxazirane is cleaved 
via a concerted process with concurrent migration of one of the groups on the $\alpha$-carbon of the N-alkyl substituent. In the transition state for this reaction, the nitrogen is thought to be electron-deficient, thus providing the driving force for a 1,2 carbonium-ion type migration. The observed migratory aptitude was $\phi>$ methyl $>\mathrm{H}$, characteristic of migration to an electron-deficient site. In the absence of reaction by heterolytic cleavage of 3-phenyloxazirane, one would not expect a relation between the ionizing power of the solvents and the efficiency of the transformation. Splitter and Calvin $(67 \mathrm{~b}, \mathrm{c})$ have suggested that in protonic solvents, rearrangement by a or a' was favoured, primarily on the grounds that a preponderance of formanilides instead of the corresponding benzamides was produced from the 2,3diphenyloxaziranes. In the present instance, the exclusive formation of benzamides would imply that protonic solvents or acids are probably unimportant in the rearrangement of 3phenyloxaziranes to amides. If $a, a^{l}, b$ and $b^{l}$ are improbable, other possibilities such as $c, c^{I}$, and $d$ may be considered. Process $c, d(1,2$-free radical shift) in which the migration of hydrogen atom occurs in step-wise fashion is considered improbable in the absence of any authenticated example in the electronic ground state chemistry of free radicals $(34,35)$. Pathway $c$ differs from $c^{I}$ in that the latter is a concerted process in which attainment of a neutral state by a rapid electron reorganization and synchronous migration of hydrogen provides the driving force for the final conversion of the 
intermediate to a benzamide. A totally concerted mechanism of type d is possible in which the reaction would be of the "no mechan1sm" type (215). In the rearrangement, however, the stereochemistry of the oxazirane requires rotation about the C-N single bond for the migrating group (H) to enter from the rear side of the leaving oxygen as the N-O bond cleaves. Thus, considerable stretching of the N-O bondin $123 \mathrm{~b}$. will be

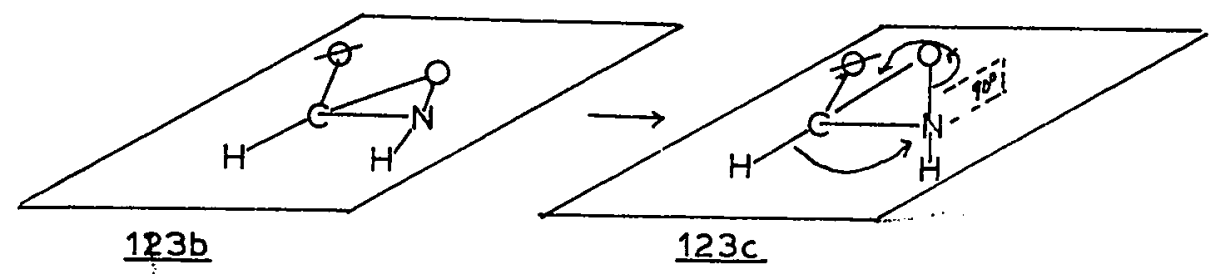

required in the transition state and conversion to the benzamlde through a highly energetic state might be an unfavourable pathway $\nabla 1 a \underline{123 b} \rightarrow 123 \mathrm{c}$. Although a "no mechanlsm" reaction for 3-phenyloxazirane decomposition would certainly simplify the interpretation of the effects of substituents, solvents, and acids on the over-all transformation, there are two important pieces of evidence to show that conversion of the intermediate may involve $c$ process rather than process d. The first of these is the nature of the reaction in dioxane. Irradiation of benzaldoxime in dioxane gives an extremely low jield of benzamide. Interaction between the solvent and a species capable of initiating a free radical reaction by abstraction of hydrogen from the dioxane may be responsible. In the residue of the photolyte (dioxane and monoglyme), the most 1ntense carbonyl absorption is at 1728$1730 \mathrm{~cm}^{-1}$, which is consistent with the possible formation of an aldehyde (219). This abserption 18 definitely not due to 
the acetylated product of benzaldoxime since its carbonyl absorption appears at $1770 \mathrm{~cm}^{-1}$. Initiation of the free radical reaction of dioxane certainly could not be caused by absorption of light by the solvent since the solvent 18 transparent at $290 \mathrm{mu}$. Irradiation of benzaldoxime in dioxane with unfiltered light led to a residue containing the same carbonyl absorption at $1730 \mathrm{~cm}^{-1}$, and in the n.m.r. spectrum, methylene proton absorption was apparent, indicative of the diozane molety in the product. The quenching of benzamide In the presence of oxygen 18 also consistent with the intervention of a diradical intermediate since the reaction between the diradical species and oxygen would be expected to interfere with the final conversion of the oxazirane to benzamide.

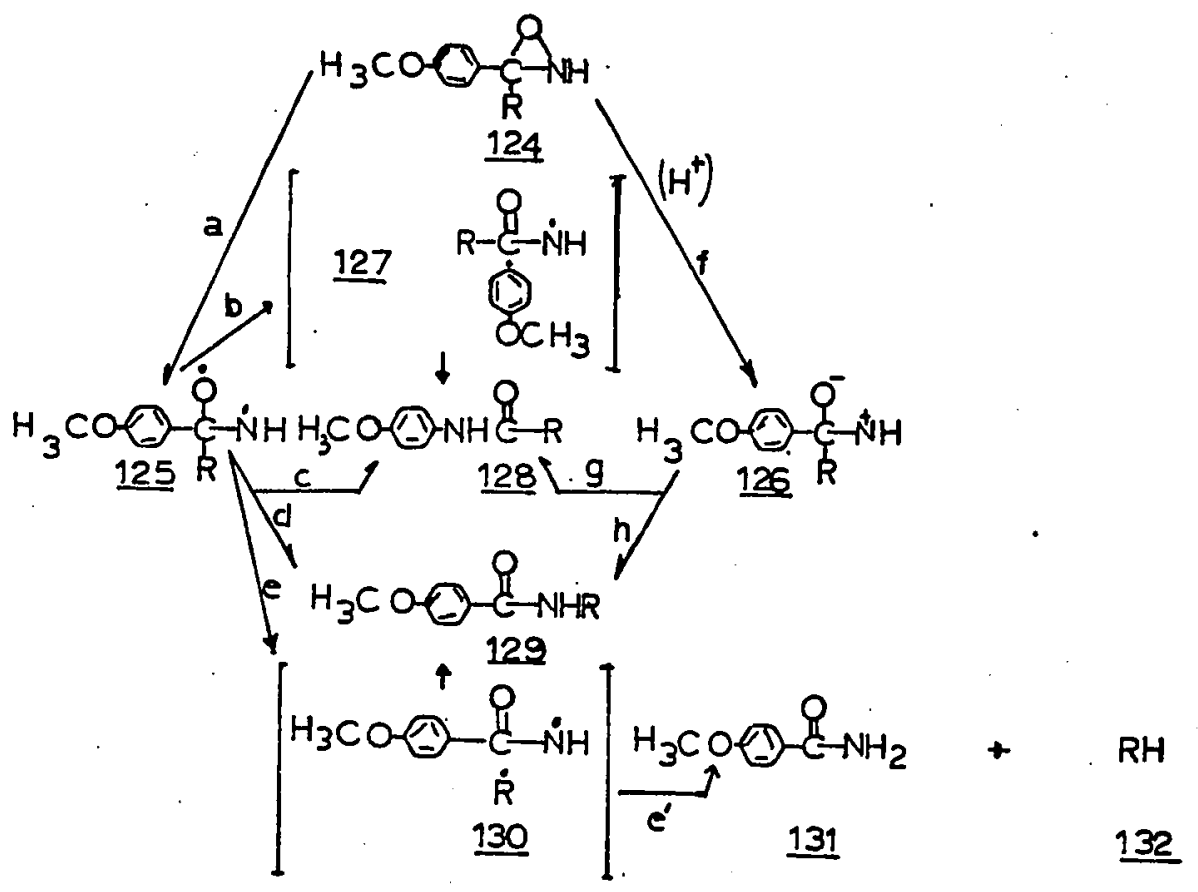


For benzaldoximes, the present data does not distinguish between a strictly intramolecular 1,2 migration from (124, $\mathrm{R}=\mathrm{H})$ to (129, $\mathrm{R}=\mathrm{H}$ ) and a fragmentation-recombination process. However, for p-methoxyacetophenone oxime, ( $\left.124, \mathrm{R}=\mathrm{CH}_{3}\right)$ which rearranges presumably through 3-anisyloxazirane, the mechanism might be entirely different. This was evident from the isolation of a demethylated product, p-anisamide from irradiation of the ketoxime. Methane might have been formed but no attempt was made to detect it. Similar mechanism invoking the intervention of a radical-pair, 127 is unlikely, on the ground that anisole or acetamide could not be detected in the products. RecentIy, Taylor and spencer (216) observed that 6-methyl- and 6-phenyl-phenanthridine-5-oxide 133 are photochemically rearranged to the corresponding 5-methyl- and 5phenyl-6-(5H) phenanthridines 135: presumably via an oxazirane. In this instance both methyl and phenyl groups were shown to migrate.

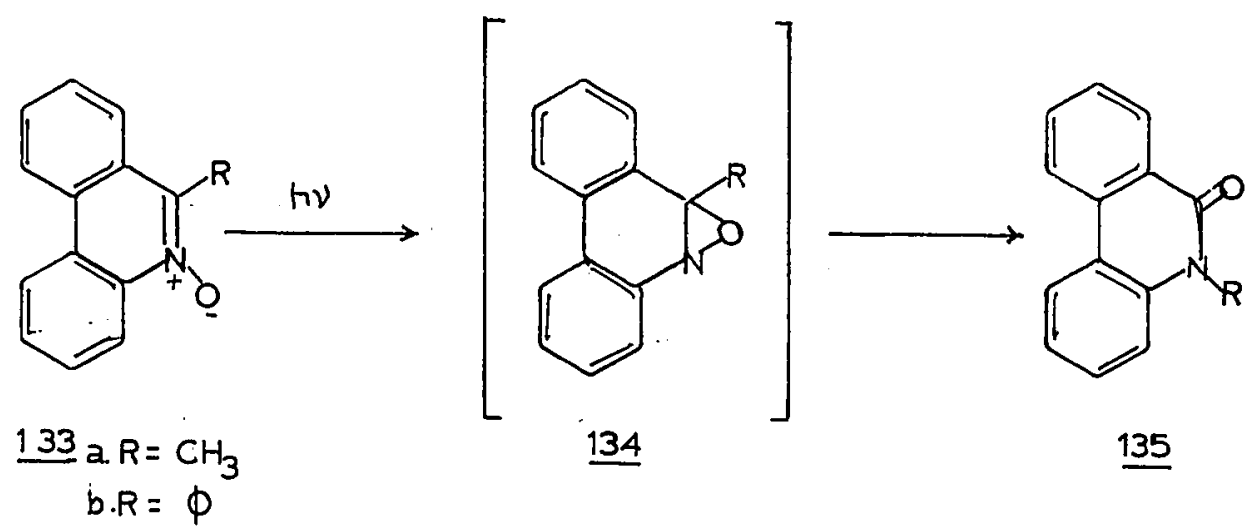

Reaction 36 
In the rearrangement of 3-hengbxazirane, an analogous mode of transformation may occur with the intervention of a diradical intermediate, 125 in which the migration of a group (methyl, hydrogen or phenyl) is envisaged as a movement to a radical site or to an incipient radical site. Ejection of a radical from the $\alpha$-carbon results in the formation of a $C=0$ bond. In the rearrangement of $\beta$-methyl- $\beta$-phenylperoxy propiolactone 136, Greene and co-workers (220) have argued that the critical factor which determines the migration aptitude may be related to the difference in the ease of dissociation of the methyl and phenyl group from the sites of attachments. Such a rationalization of the results can also apply to the exclusive formation of benzamides upon irradiation of benzaldoxime, via the 3-phenyloxaziranes, since the dissociation between $H$ and phenyl has been estimated to be about 12k.cal/ mole (22la). Pertinent to the argument are the following dissociation bond energies:

$$
\begin{aligned}
& D_{\mathrm{CCH}_{2}}-\mathrm{H}=80 \mathrm{k} \cdot \mathrm{cal} \cdot \mathrm{N}^{-1}, \mathrm{D}_{\phi}-\mathrm{CH}_{2} \mathrm{CH}_{3}=92 \mathrm{k} \cdot \mathrm{cal} \cdot \mathrm{M}^{-1}, \\
& { }_{\mathrm{C}_{\mathrm{CH}_{2}}-\mathrm{CH}_{3}}=65 \mathrm{k} \cdot \mathrm{cal} \cdot \mathrm{N}^{-1}
\end{aligned}
$$

Uncommon as 1,2-shifts of higdrogen and alkyl groups are in free radicals except for apparent photochemically induced rearrangements $(29,33,34)$, Greene and co-workers (220) have recently reported that thermally induced rearrangement of $\beta$-methyl- $\beta$-phenylperoxypropiolacetone 136 , yields both propiophenone 140 and benzyl methyl ketone $\underline{141}$ in a ratio of $5: 1$, in which there is a preponderance of apparent 1,2methyl migration. To account for these results, Greene and 
co-workers have suggested that, as one of the possible

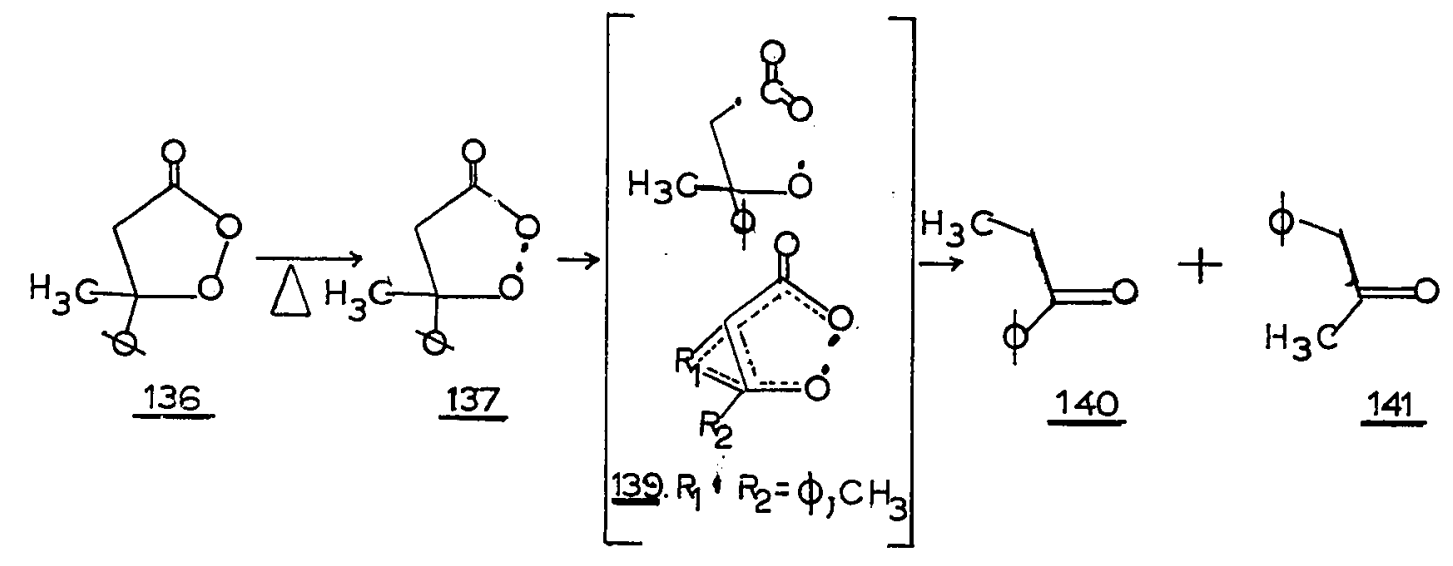

Beaction 37

mechanistic pathways, the rearrangement from 136 to 140 and 141 possibly involves a rapid decarbonylation of 137 to 138 , followed by a rapid but synchronous rearrangement of the diradical 138 in which the migratory aptitude uncommon to 1,2-free radical or 1,2 carbonium ion shifts is observed. Kharash (12a) has reported an analogous reaction. He observed a preferential extrusion of an alkyl group rather than phenyl group for a cumyloxy radical, the reaction being:

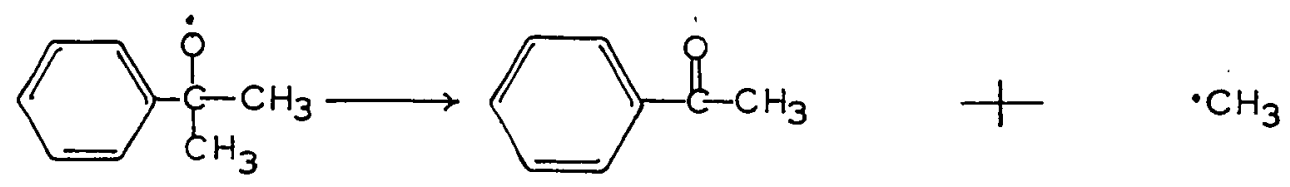

$\underline{\text { Reaction } 38}$

The preceding argument invoking the differences in the dissociation energies of the migrating groups eannot be satisfactorily applied to the rearrangement of p-methoxyacetophenone since comparable migratory aptitudes of the 
anisyl group, methyl group was observed (see Table $X$ ). One would expect to find greater differences in the migratory aptitudes of these groups than that for an unsubstituted phenyl group in the reaction involving a free radical or the intervention of an incipient free radical. The migration of an anisyl group via a free radical intermediate might not be expected to be favourable in view of Ruchardt's work (222). He reported that the relative migratory aptitudes of the anisyl and phenyl groups are 1:3. This electron-donating substituents on the phenyl ring retard migration of phenyl groups in a 1,2-free radieal rearrangement. A plot of the rates of reaction vs the Hammett's Sigma function gives a positive rho value. It is of interest that Emmons (78b) suggested that pyrolytic rearrangement of alkyl oxaziranes proceeds through diradicals of type 125 (Scheme VIII). In one particular instance, 2-t-butyl-3-isopropyloxazirane 142 pyrolyzed at $250^{\circ}$, leads to exclusive migration of the hydrogen with the formation of $\mathbb{N}$-t-butylisobutyramide 143 .

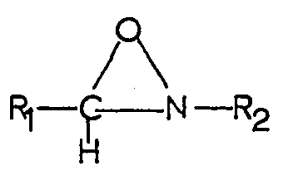

142. $R_{1}=\mathrm{i}-\mathrm{C}_{3} \mathrm{H}_{7}$ $R_{2}=\mathrm{i}-\mathrm{C}_{4} \mathrm{H}_{8}$
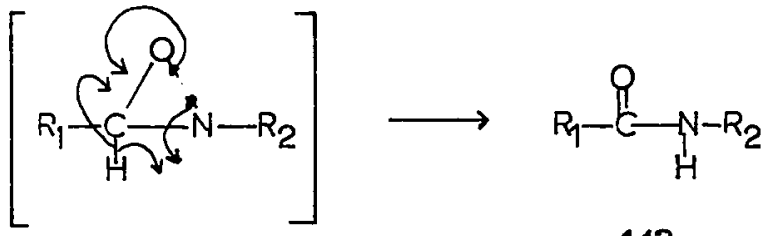

143

\section{Reaction 39}

Photochemical isomerization of p-anisaldoxime and pmethoxyacetophenone oxime to the corresponding amides gave 
unexpected results in comparison with all the other benzaldoximes which were studied. For 2,3-diphenyloxaziranes, generally, heterolytic ring fission of the N-O bond has been found to be favoured in oxaziranes substituted on the 3phenyl group by electron donating substituents. In Table IX, the results show: that the migratory aptitude of an anisyl group is slightly greater than that of a hydrogen atom. Therefore, the observed result for the oximes could reflect either the change in the mechanism or the introduction of a competing mechanistic pathway leading to a mixture of products containing amides having an apparent migration of an anisyl group as well as hydrogen or methyl group. This competitive pathway might be represented as an acid-catalyzed rearrangement of oxazirane to the amides.

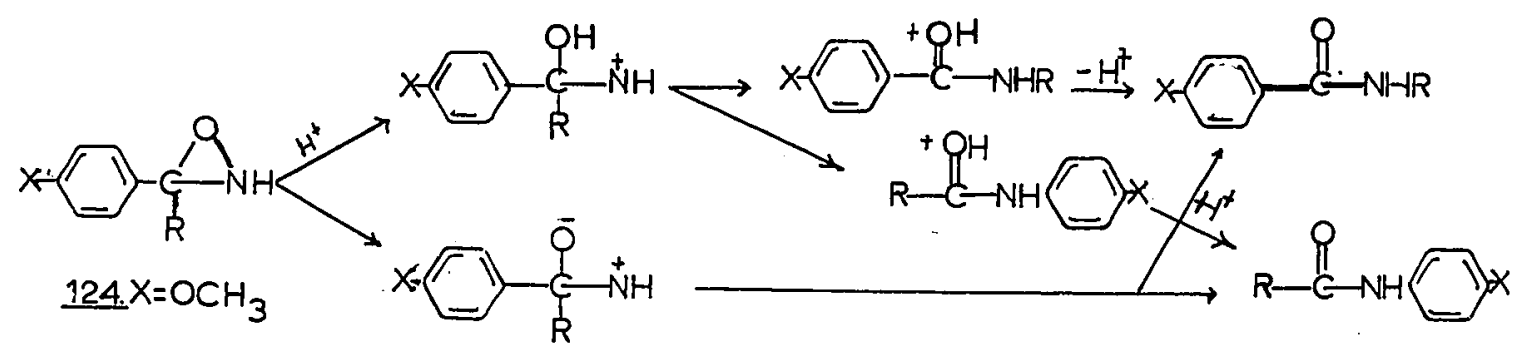

Reaction 40

Possible competing reactions are also given in Scheme VIII. It has been suggested that in polar solvents such as ethanol, 
ionic rearrangement involving both hydride and phenyl migration can compete favourably, depending on the acidity of the medium (223).

Recently, Kan, (224) has suggested a free-radical chain reaction as an alternative mechanism represented by the following series of reactions:
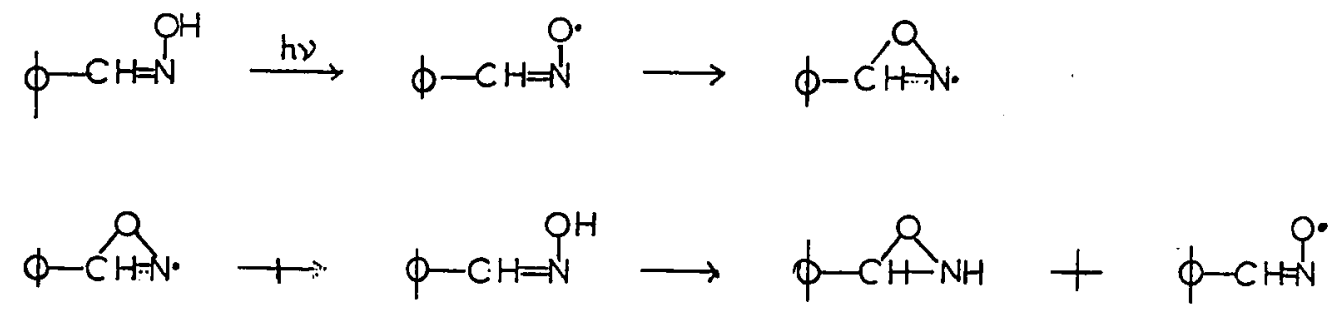<smiles>CCCCC(=O)C1NO1</smiles>

Scheme IX

This representation of the photochemical Beckmann rearrangement of aryl aldoximes to benzamides must be excluded in the light of the present results. A free-radical chain reaction would imply that the quantum yield of the reaction should be extremely high. In fact, without addition of acid, the quantum yield was only 0.002 . The participation of the proton is completely excluded in the scheme, but experimental results clearly indicate that in its absence, the quantum efficiency is extremely poor. Furthermore, despite the 
decrease in electron density along the $0-\mathrm{H}$ bond which occurs on excitation to the singlet state, a homolytic cleavage of the aldehydic hydrogen (6-H) rather than a homolytic cleavage of the $\mathrm{O}-\mathrm{H}$ bond would be expected if a reaction could have been initiated by a radical-chain reaction. The dissociation energy of the $\mathrm{O} H$ bond is about $102 \mathrm{k.cal} . / \mathrm{mole}$ (221b), whereas that of the $\mathrm{C}-\mathrm{H}$ bond of benzaldehyde is only about $87 \mathrm{k.cal} . / \mathrm{mole}$ (22la). Dissociation of the $\mathrm{N}-\mathrm{O}$ bond would be most probable since the dissociation energy has been reported as 39-6I k.cal./mole. In Scheme IX, the multiplicity of the states involved in the rearrangement was also ignored.

\section{Summary:}

In the light of the present investigation the mechanism of the photochemical Beckmann rearrangement of benzaldoximes may be represented as in scheme $X$. Absorption of light causes excitation of the singlet state, and in the absence of any proton source, radiationless transitions by internal conversion (IC) and intersystem crossing (ISC) are proposed as the important fates of the radiant energy. The latter process is considered to be responsible for the syn-anti or cis-trans isomerization via the intervention of triplets, 146 and 1461. These same triplets can be reached by collisional sensitization. The triplet-triplet annihilation processes are included in the scheme since a small fraction of the triplet reverts to the singlet state. The importance of the protonation in the excited singlet is favoured rather than the 

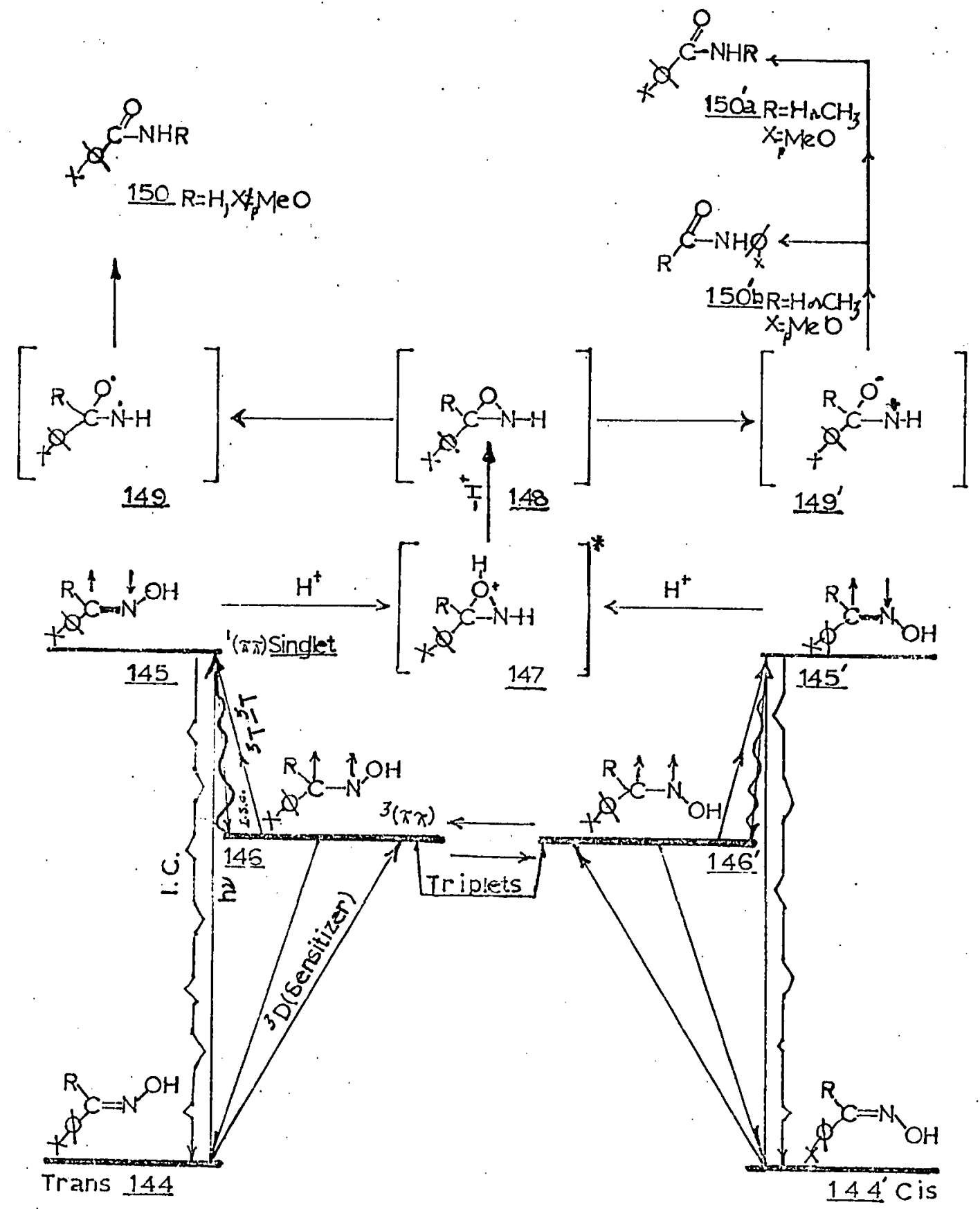

Scheme X

Mechanism of the Photochemical Beclmann Rearrangement 
step involving the transformation of the 3-phenyloxazirane in view of the theoretical calculation of the change in the pKa value for the protonated form of benzaldoxime in the excited singlet. Considering that the nitrogen of the oxime can be readily protonated by strong acids even in the electronic ground state, an increased electron density at nitrogen upon excitation would be expected to lead to a rapid reaction of the oxime with a proton sorrce. Protonation of the oxime in the excited state is shown as leading to the excited protonated form 147 of the oxazirane, which in turn affords the oxazirane in the electronic ground state 148. The general scheme includes the effect of electron-donating substituents on the rearrangement. For 3-p-anisyloxazirane and 3,p-anisyl-3-methyloxazirane, a heterolytic cleavage is favoured over a free diradical cleavage. The latter mode of rearrangement is suggested for the other benzaldoximes which were studied. Species 149 could, perhaps, be better represented as the protonated form under acidic conditions. 


\section{EXPERTMENTAI}

A. Materials

I. Preparation of Benzaldoxime, p-Substituted Benzaldoximes, and p-Methoxyacetophenone oxime

1. Materials:

Hydroxylamine hydrochloride and sodium acetate (Fisher, reagents) were used without further purification. p-Tolualdehyde, p-bromobenzaldehyde, p-chlorobenzaldehyde (K\&K), p-fluorobenzaldehyde (Aldrich), p-anisaldehyde (City

Chemicals) and p-methoxyacetophenone (Eastman Organic Chemicals) were also used without further purification for the preparation of their p-substituted benzaldoximes. Benzaldehyde $(\mathrm{BDH})$ was washed with an aqueous solution of sodium bicarbonate, extracted with ethyl ether, dried over anhydrous magnesium sulfate, and distilled under nitrogen (bp.179-180 , lit. $\left.179.5^{\circ}\right)$. p-Methoxyacetophenone oxime was similarly prepared from p-methoxyacetophenone (Eastman Organic Chemicals).

\section{Methods of Preparation:}
a) $\alpha$-Isomer:

All the $\alpha$-benzaldoximes were prepared by a siight modification of the method described in reference (225), utilizing sodium acetate. Because a rapid preparation of some of the $\alpha$ - and $\beta$-benzaldoximes by the conventional methods was 
not entirely satisfactory, a method generally applicable to simple, quick preparations of the two isomers was developed.

To an aqueous solution ( $150 \mathrm{mI}$ ) of hydroxylamine hydrochloride $(0.7 \mathrm{M})$ and sodium acetate (IM) benzaldehyde (0.5M) was added and the resulting mixture was heated to $40^{\circ} \mathrm{C}$, stirred vigorously at this temperature for 5-10 minutes, and then left under refrigeration for 10-12 hours. Ethanol and ethyl ether were frequently suitable for dissolving solid benzaldehydes. The product was extracted with ethyl ether ( $150 \mathrm{mI})$, washed with water, dried over anhydrous magnesium sulfate, and subsequently concentrated in vacuo' (15-20 min.) with gentle heating. The crude ethereal extract was purified either by distillation or crystallization, or by thin layer chromatography followed by distillation or crystallization from a suitable solvent.

A large scale separation of $\alpha$-isomer from $\beta$-isomer and contaminating substances was very readily effected by thin layer chromatography. Crude $\alpha$-isomer (250mg) was applied to a plate (8" $x$ 8") coated with a layer (3/16"-1/4" thick) of silica gel (60gm) (CF254 or Kieselgel) and eluted with a solvent mixture of either cyclohexane and ethyl ether $(4: 1)$ or petroleum ether $\left(35-60^{\circ}\right)$ and ethyl ether $(4: 1)$. The two isomers separated on the chromatogram were scraped off, extracted with ethyl ether, filtered, and concentrated by 
gentle heating. It was necessary to aroid excess heating during the evaporation of solvents in order to prevent geometrical isomerization.

$\beta$-Isomers, on the other hand, were prepared by two methods. The more suitable method was selected.

\section{b) $\beta$-Isomers:}

\section{i) Photochemical Process:}

$\alpha, p-S u b s t i t u t e d$ benzaldoxime $(1-5 \mathrm{gm})$ was first dissolved in cyclohexane $(200 \mathrm{ml})$ and irradiated in a vessel through a quartz immersion well with a 85 watt medium pressure mercury lamp for 1-3 hours. The crude $\beta$-isomer which crystallized out from the solvent system was filtered, and recrystallized from an appropriate solvent system. When the $\alpha$-isomer was not readily soluble in cyclohexane, a saturated solution at its reflux temperature was irradiated for $1 / 2$ hour and then cooled to $10-15^{\circ} \mathrm{C}$ by circulating cold water through the cooling envelope of the immersion well. This last operation facilitated the precipitation of the $\beta$-form which is far less soluble in hydrocarbon solvents than is the $\alpha$-isomer. When necessary the $\beta$-form was purified by thin layer chromatography followed by recrystallizations but in most cases where this method was applicable, only one recrystallization from ethyl ether and cyclohexane was necessary for purification of $\beta$-benzaldoximes. 


\section{ii) Chemical Process:}

The method followed was essentially that described by Vogel (ref. 163, p.791) with one important modification in the general scheme. Rather than purifying the $\beta$-isomers by several crystallizations it was found in our laboratories that a very rapid preparation of the $\beta$-isomers could be made by TIC separation of the crude products followed by crystallization from ethyl ether and cyclohexane. The $\alpha$-isomer was initially converted into the $\alpha$-form by bubbling a slow stream of anhydrous hydrogen chloride gas through an anhydrous ethereal solution. The oxime salts which separated out were reconverted to a mixture of $\alpha$-and $\beta$-isomers by treatment with an aqueous solution of sodium carbonate and then extracted with ethyl ether. The ethereal extract, after concentration 'in vacuo' was separated by TIC and worked up by the method already described under section

As a precaution, a small amount of the purified forms was rechromatographed on the plate and inspected under ultraviolet light in order to check the purity of the samples.

This second method was suitable for the preparation of halogenated $\beta$-benzaldoximes.

Benzaldoxime:- The crude product, obtained cby evaporation of ethyl ether, was distilled 'in vacuo' at $74-75^{\circ} / \mathrm{lmm}$, and 
crystallized after standing at room temperature for a period of two weeks $\left(m p .32-34^{\circ}\right.$, Lit. $\left.35^{\circ}\right)$. The yield was $80 \%$. However, separation by TIC and subsequent distillation at $(60-100 \% .2 \mathrm{~m})$ led to a crystalline product almost jmmediately $\left(m p .35^{\circ}\right)$. This procedure is highly recommended for preparation of $\alpha$-form .

$\beta$-Benzaldoxime was prepared photochemically and crystallized from benzene and cyclohexane (mp.129-130 $\left.130^{\circ}\right)$. Yield was $80 \%$. The conventional preparative method recommended in reference (163) was not reproducible.

p-Tolualdoxime:- $\alpha-p-T o l u a l d o x i m e$ was purified by TIC (eluent was a mixture of ethyl ether and cyclohexane $(1: 4)$ )

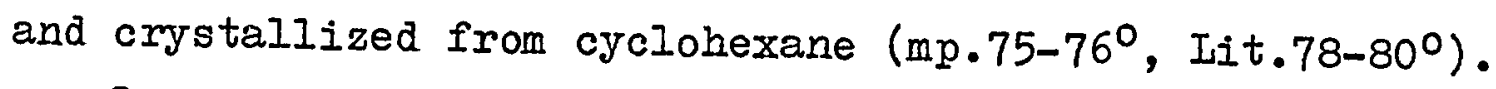
The $\beta$-isomer was prepared photochemically and crystallized

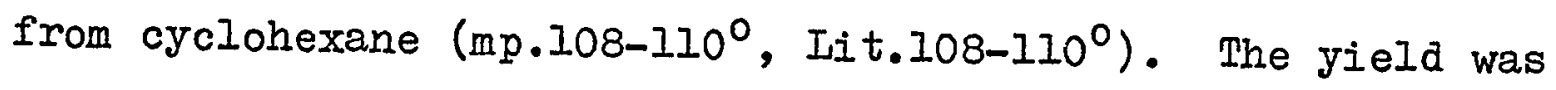
$80 \%$.

p-Anisaldoxime:- $\alpha-p-$ Anisaldoxime was separated from impurities and traces of $\beta$-isomer by mIC (eluent was a mixture of petroleum ether (35-60) and ethyl ether (3:1)). Crystallization from chloroform yielded a pure sample (mp.61-620, Iit. $\left.61^{0}\right)$. An especially effective method was to distil the $\alpha$-form in a "bulb-to-bulb" apparatus at 60-80\% $1 \mathrm{~mm}$. The $\beta$-isomer was prepared photociemically at the reflux temperature of the solvent, and crystallized from ethanol and 
benzene $\left(m p .131-132^{\circ}\right.$, Iit. $\left.132^{\circ}, 133^{\circ}\right)$. The yield was $80 \%$.

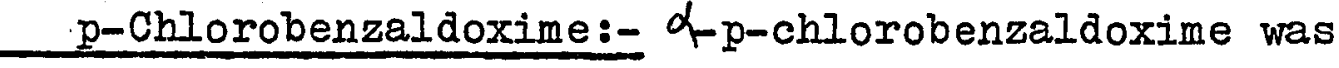

purified by crystallization from ethyl ether and petroleum

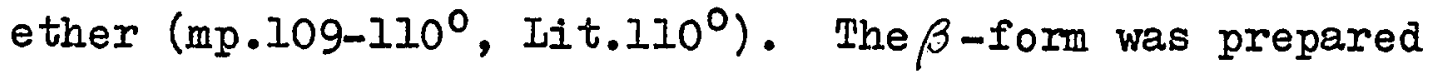
photochemically at the reflux temperature of cyclohexane and crystallized $4 \mathrm{X}$ from cyclohexane and ethyl ether (mp.145-1460 $\mathrm{C}$, Iit. $\left.146^{\circ}\right)$. The yield was $67 \%$. A more convenient method was to separate the $\beta$-isomer from the $\alpha$-form by TIC (ethyl ether and cyclohexane $1: I$ ) and then to crystallize only once from ethyl ether and cyclohexane (mp.146-147 ${ }^{\circ}$, Lit.146 ${ }^{\circ}$ ).

p-Bromobenzaldoxime:- $\alpha-p$-Bromobenzaldoxime was purified by TLC (eluent, ethyl ether and cyclohexane 1:I) followed by crystalization from cyclohexane and ethyl ether (mp.112-1130, Lit. $\left.110^{\circ}\right)$. The $\beta$-isomer was prepared by treatment of the $\alpha$-form with anhydrous hydrochloride gas. Initial separation of the $\beta$-form from the $\alpha$-oxime was accomplished by TIC, and subsequent crystallization from ethyl ether and cyclohexane

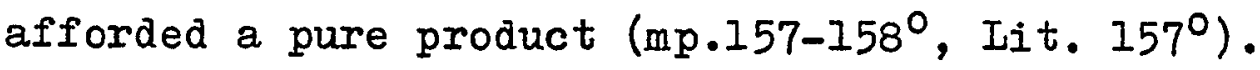

p-Fluorobenzaldoxime:- Pure $\alpha$-p-fluorobenzaldoxime was obtained by the crystallization from cyclohexane and ethyl

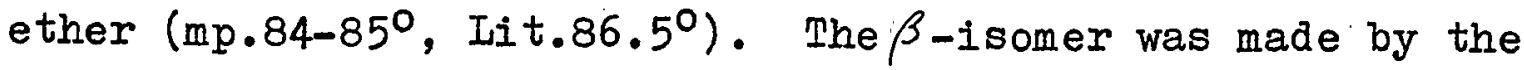
same method prist employed in the preparation of

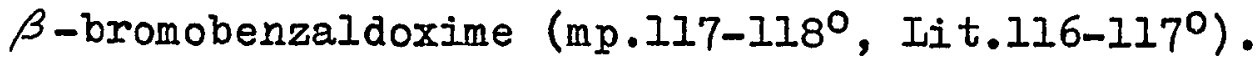




\section{Q-Methoxyacetophenone ketoxime:- $\alpha$-Methoxyacetophenone} oxime was prepared by treatment of the corresponding ketone with hydroxylamine in the manner described above for the preparation of benzaldoximes. The crude product was crystallized from a mixture of benzene and cyclohexane (mp.84-85', Lit. $\left.87^{\circ}\right)$.

\section{Preparation of Amides}

p-Anisidine, anjisic acia (Eastman Organic) and acetyl chloride (BDH) were used without further purification. Thionyl chloride (Fischer) was purified according to Fieser (225) by treatment with quinoline followed by distillation from linseed oil. Benzoyl chlorides and benzamides were either supplied by chemical companies or prepared in this laboratory according to the methods described by Vogel (ref. 163 p.755). Benzamide (BDH, p-toluamide (Eastman Organic), p-chlorobenzamide (Aldrich), and p-anisamide (City Chemicals) were purified by recrystallization from ethanol and water. The respective melting points were (125.5-126.5 , Iit.125-126年),

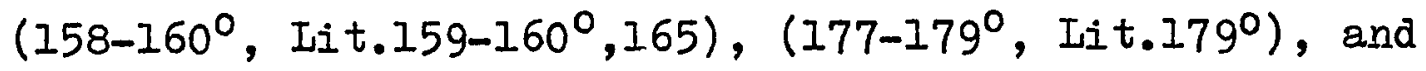

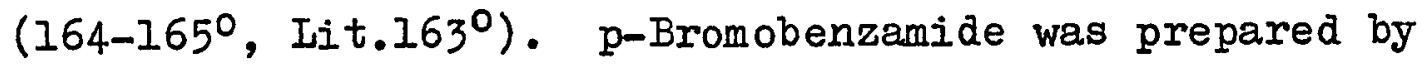
treatment of p-bromobenzoyl chloride wi th ammonia, and crystallized from water and ethanol (mp.191-1920, Lit.189190 ${ }^{\circ}$. p-Bromobenzoyl chloride was initially prepared by the reaction between p-bromobenzoic acid (mp $255^{\circ}$, Iit. 25I255), obtained by alkaline permanganate oxidation of pbromobenzaldehyde (K\&K). Similarly p-fluorobenzamide was 
prepared by ammonia treatment of p-fluorobenzoyl chloride made from p-fluorobenzoic acid (Aldrich). p-Methoxyformanilide was synthesized by the procedure of Frohlich and Wedekind (226) involving treatment of formic acid (B\&A, 98\%) with panisidine. A pure sample was obtained by crystallization from ethanol (mp.79-80 , Lit.80-8I). N-p-Anisylacetamide was prepared by reaction of p-anisidine with acetyl chloride followed by recrystallization from a solvent mixture of water and ethanol (mp.128-129 , Iit.130-135). N-methylanisamide was formed from p-anisoyl chloride and methylamine (Matheson, Coleman, and Bell). To a $40 \%$ aqueous solution of methylamine was added $40 \%$ aqueous sodium hydroxide, and the resulting mixture was warmed gently with a Bunsen burner until the gas $\left(b p=6.5^{\circ}\right)$ was evolved. The methylamine vapour was passed upwards through a column of sodium hydroxide pellets and was condensed into a $100 \mathrm{ml}$ roundbottomed flask cooled in liquid nitrogen. Then, anisogl.chloride (bp 130\%/14m.m.), prepared from the reaction of anisic acid and thionylchloride, was slowly added to $75 \mathrm{ml}$ of the condensed methylamine which had been warmed up to $-117^{\circ}$. The reaction occurred instantly and the excess methylamine was distilled off. Crystallization of the crude product from water and ethanol afforded a pure sample (mp.120-1210, Iit. 119.5-120.50).

III. Preparation of Aromatic and Ketonic Sensitizers Triphenylene (Aldrich and Columbia Organic) was chromatographed on a column of aluminium oxide by eluting with 
petroleum ether $\left(35-60^{\circ}, \mathrm{BDH}\right.$, reagent), and was recrystallized several times from a mixture of cyclohexane and benzene (mp.

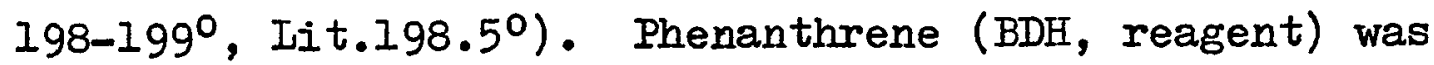
treated with maleic anhydride in order to remove traces of anthracene. Chromatography of the reaction mixture on an alumina column, followed by crystallization and sublimation

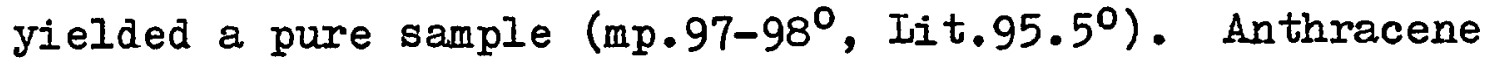
(Eastman Organic blue-violet flourescent grade) was purified by pyrolysis of the adduct of maleic anhydride according to the procedures of Vogel (ref.163, p.942) and King (227).

Benzophenone ( $\mathrm{BDH}$, reagent) was used wi thout further purification. Michler's Ketone ( $p, p$-dimethylaminobenzophenone) was recrystallized from ethanol (mp.172-173, Lit.174 ${ }^{\circ}$ ) and 4hydroxyacetophenone was recrystallized from ethyl ether (mp. 109, Iit. $\left.109^{\circ}\right)$. Acetone (Fisher, spectroscopic grade) was dried over molecular sieves and fractionally distilled. Biacetyl (Fisher, reagent) was purified by distillation on the spinning band column. The first 10ml were discarded and the fraction distilling at $88.5^{\circ} \mathrm{C}$ was collected $\left(\right.$ Iit. $8^{\circ} \mathrm{C}$ ) and stored in the dark before use.

\section{Purification of Solvents}

i) Aprotic Solvents:

Cyclohexane (Fisher, spectroscopic) was used wi thout further purification. Dioxane (Fisher, reagent) was purified according to procedures given by Fieser (ref.225, p.284) and 
Vogel (ref.167, p.177). The final purification procedure involved refluxing over, and distilling from, sodium (bp.1010, Iit.101.5 ). Monoglyome (Ansul) was refluxed over, and distilled from, calcium hydride (bp.83-84, Iit.83-84). Acetonitrile (Eastman Chemicals, Spectroscopic) was distilled successively from phosphorus pentoxide, anhydrous potassium carbonate and finally phosphorous pentoxide (bp.81-82 ${ }^{\circ}$, Iit. $\left.82^{\circ}\right)$. (ref.163, p.407,228,229). Acetone (Fisher, Spectroscopic) was dried over molecular sieves (1/10" pellets, Type 4A) for three days, percolated down a column (21) of molecular sieves, and then distilled (bp.55-56 ${ }^{\circ}$, Iit.56.5) $(228,230)$. Petroleum-ether $\left(35-60^{\circ}\right)$ (BDH, Analar) was distilled on a spinning band column, and anhydrous ethyl ether (Mallinkrodt) was employed for chromatography without further treatment.

\section{ii) Protonic Solvents:}

Acetic acid (Analar reagent) was used without further treatment. Absolute methanol and ethanol were purified by Vogel's procedure (ref.163, p.169), by drying them over anhydrous calcium oxide which had been heated in a muffle oven for one day at $600^{\circ} \mathrm{C}$, and then distilling from the corresponding magnesium alkoxides. (Methanol, bp.64.2, Iit.64.7, ethanol, bp.78 , Lit.78.5). Water was distilled from a slightly acidic solution of ceric sulphate and redistilled from a weak solution of sodium hydroxide. An $80 \%$ aqueous ethanolic solution was made up according to Winstein (228) from a mixture of ethanol (15.80g) and water (5.00g). A 50\% 
solution was prepared in a similar way. Isopropyl alcohol (Fisher, reagent) was dried over anhydrous calcium oxide and distilled from calcium as prescribed by Vogel (ref.163, p.168)

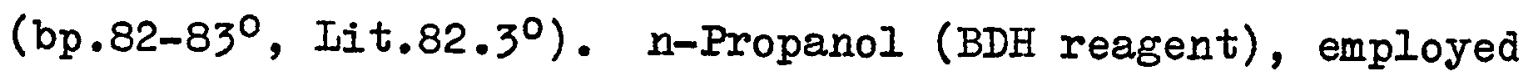
as solvent for ultraviolet spectrophotometry, was used without further purification.

V. Organic Acids Selected for Irradiation Studies

Acetic acid (BDH, Analar) was used without further purification. Trifluoroacetic acid (Anachemia) was redistilled before being used in any series of experiments (bp.720, Iit. $\left.72.5^{\circ}\right)$

VI. Materials Selected for Phosphorescence Studies

Benzophenone and triphenylene were selected as sensitizers for experiments designed to study the effect of quenchers ( $\alpha$ - and $\beta$-benzaldoxime) on the phosphorescence of a sensitizer at $77^{\circ} \mathrm{K}$. Biacetyl was selected for investigation at room temperature. EPA (a mixture of ethyl alcohol, isopentane, and ethyl ether in the ratio of 5:5:2) was used as the solvent suitable for forming a glass at the temperature of liquid nitrogen. EPA was supplied by American Instrument Co. Inc., and used without further treatment.

VII. Materials Selected for Irradiation of p-Anisaldoxime at Low Temperature

n-Hexane (BDH, spectroscopic) was selected as the solvent 
for irradiation of p-anisaldoxime at temperatures between $-90^{\circ}$ to $-50^{\circ} \mathrm{C}$. MCP, a $4: 1$ mixture of methylcyclohexane and n-pentane, was selected for irradiation at $77^{\circ} \mathrm{K}$. The latter solvent was supplied by American Instrument Co. Inc. Both n-hexane and MCP were used wi thout further purification.

Phenyl isocyanate, (Matheson, Coleman and Bell) chosen as a reagent for trapping oxazirane at low temperatures, was dried over, and distilled from, sodium hydride (23I), (bp. $55 \% /$ m m, Lit.55\%/I3m.m.). After the distillation, 2501 samples were transferred into small ampoules, sealed, and stored until required. Manipulation of the phenyl isocyanate was confined to the drybox.

\section{B. The Measurements of Quantum Yields I. Apparatus Set-Up and Iight Sources}

The split-beam irradiation apparatus schematically represented in Fig. 19 was set up for the determination of the quantum yields of benzamide.

The light source for irradiation at $210 \mathrm{~m} \mu$ was a Hanovia 150 watt D.C. Xenon Compact Arc. Iamp and the source at $254 \mathrm{~m} \mu$ was a Thermal Syndicate 40 watt low prossure mercury vapour $\operatorname{lam}_{2} \mathrm{~T} / \mathrm{M} / 5 / 544 \mathrm{~A}$. Osram $\mathrm{HBO}-200$ watt high pressure mercury vapour lamps, type $I_{1}$ and $I_{2}$ provided the necessary intensity for irradiation at $290 \mathrm{~m} \mu$ and $334 \mathrm{~m} \mu$. 
An optical system was designed to provide a collimated beam split Into two beams at right angles, one of them falling on the photolyte and the other on the actinometer solution monitoring the intensity of incident monochromatic light. The light from a source was collected on a fused plano-convex lens (Esco product, O.D., I $\frac{1}{2} "$, f.I.=3") and passed down the optical bench (Ealing, No. 22-704) through a diaphram, a filter, another lens, and finally on the beam splitter (fused ground and polished quartz plate, General Electric, C/P 101, 4"x4"xl/8"! and Esco Product 3"x3"xl/16"). The intensity of the monochromatic beam was monitored on the reflected side of the beam splitter employing a chemical actinometer, and the reaction vessel or photolyte was exposed to the incident beam transmitted through the quarts beam splitter.

The block, housing the beam splitter and the reaction vessels and cut out of brass with a vertical slit $1 / 8$ " wide inclined at $45^{\circ}$ to the incident beam, represented the beam splitter unit. Internally threaded cylinders, fixed to the block, accommodated the irradiation cells. Diagram of the irradiation block is given in Fig. 20.

II. CelIs

Fused quartz cells of $5.0 \mathrm{ml}$ capacity (O.D.,28mm. P.I., 10.00m, Type S-18-120), purchased from Pyrocell Co. Ltd., were used. The cells containing the photolyte were provided 


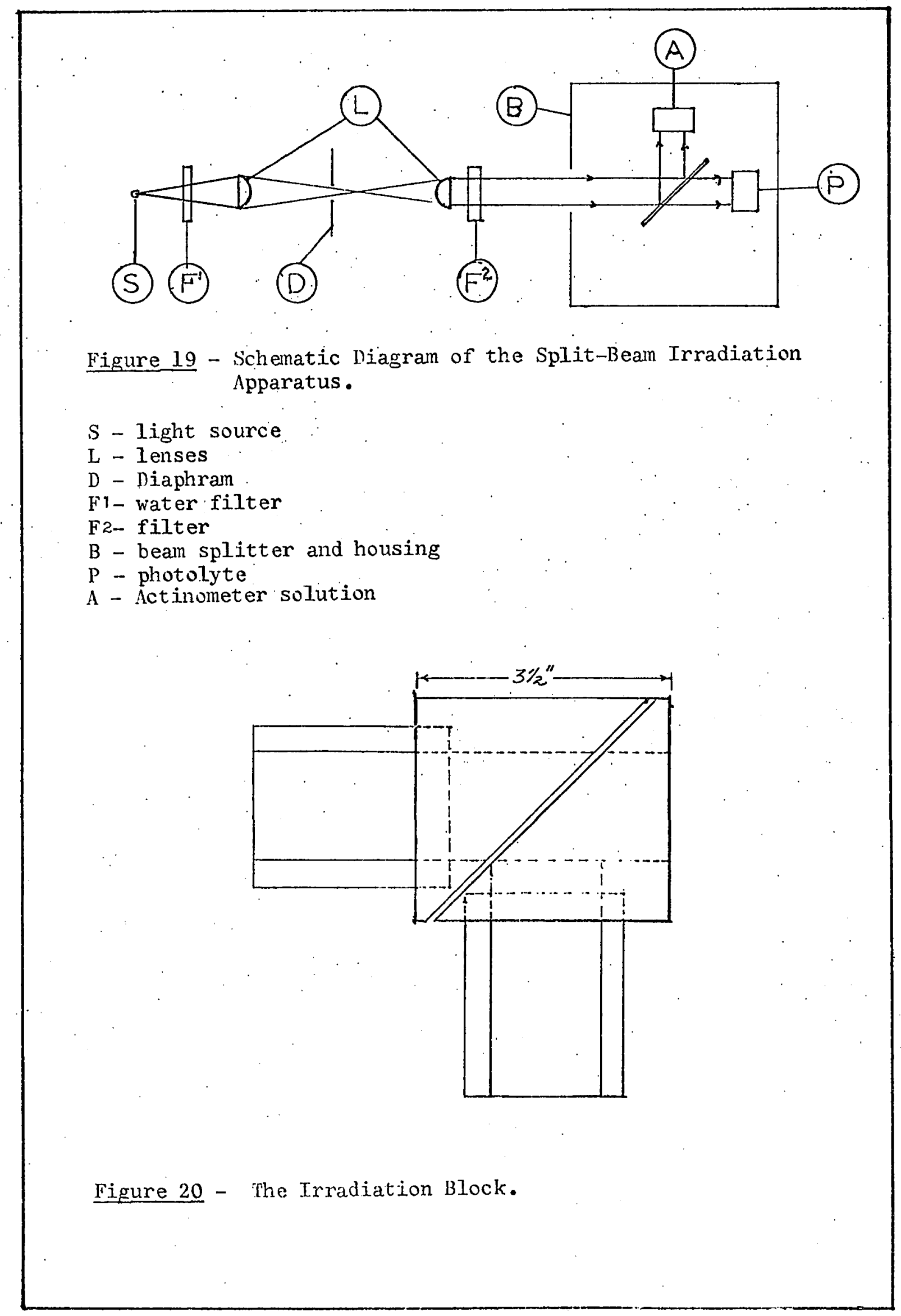




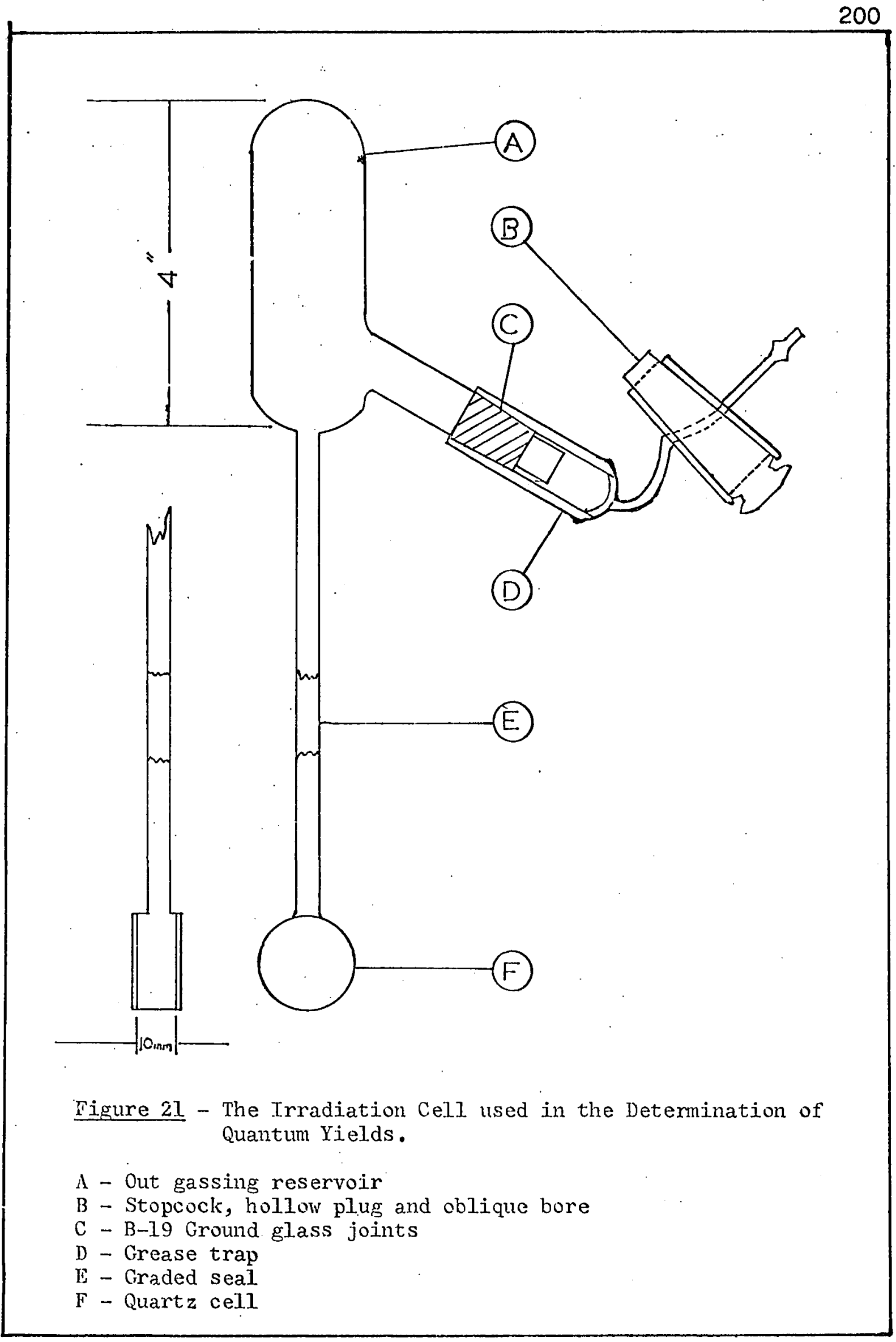


with a pyrex-quartz graded seal which could be fused to a pyrex degassing apparatus (Fig. 21). The degassing unit was constructed so as to the accommodated with a grease trap and a stop-cock capable of withstanding evacuation to a pressure of $4 \times 10^{-4} \mathrm{~mm}$ of mercury. These cells or irradiation reservoirs were incapable of withstanding" freeze-pump-thaw "operation of degassing when they were filled with dioxane and acetic acid. They also were incapable of withstanding temperatures as low as $77^{\circ} \mathrm{K}$.

In certain instances, the irradiation cells were permanently sealed under vacuum with the cells cooled in dryice-acetone bath $\left(-57^{\circ} \mathrm{C}\right)$. This could not be done in the case of dioxane.

\section{Filter System}

The filter systems in this work consisted of $1.00 \mathrm{~cm}$ path length solutions of chemical absorption filters, corning absorption filters and Baird Atomic Interference filters, A water-filled cell of $1.00 \mathrm{~cm}$ path length was always used to remove the infrared light from the HBO-200W. high pressure mercury lamp.

Most of the quantum yield measurements were made at $290 \mathrm{~m} \mu$. A beam emitted from an Osram HBO-200 watt mercury vapour lamp was filtered through a Baird Atomic interference filter (2300-3400), half band width, 25mu). 
In order to filter out the $334 \mathrm{~m} \mu$ emission line from the Osram HBO-200 W. lamp $1.00 \mathrm{~cm}$ of a saturated solution of naphthalene in cyclohexane, $1.00 \mathrm{~cm}$. of an aqueous solution of nickel hexahydrate $\left(\mathrm{NiSO}_{4} \cdot 6 \mathrm{H}_{20}, 0.5 \mathrm{gm} \mathrm{ml}^{-1}\right.$ ) and a Corning absorption filter $(0-7-51,5 \mathrm{~mm})$ were lined up in series. Peak transmission at $338 \mathrm{~m} \mu$ was $30 \%$, ( $\frac{1}{2}$ band width, 25m $\mu$ ). Transmission at $334 \mathrm{~m} \mu$ was $28.8 \%$. The transmission of the latter two filters at $334 \mathrm{~m} \mu$ was $35.7 \%$. The former filter alone (mapthalenecyclohexane) was opaque to light below $325 \mathrm{~m} \mu$, and the substrates absorbing the lines above 325ywere transparent to lines above $366 \mathrm{m \mu}$; thus estimation of the flux at $334 \mathrm{~m} \mu$ without the latter two filters could be estimated. The transmission of the half-band width quoted for the filter system at $334 \mathrm{~m} \mu$ is calculated from data obtained for the former two filters and data available in the corning Filter Brochure, Corning, N.Y. Pertinent data for the filters systems at $335 \mathrm{~m} \mu$ are given in Fig. 23.

For irradiation at $210 \mathrm{~m} \mu$, a Baird Atomic interference filter \#2150 (peak transmission 16.5\%, half band width, 10m $\mu$ ) was used. For the isolation of the mercury resonance line at $253.7 \mathrm{~m} \mu$ (232) a combination of filters was utilized. These filters were $1.00 \mathrm{~cm}$ aqueous solution of $\mathrm{CoSO}_{4} \cdot 7 \mathrm{H}_{2} \mathrm{O}(0.13 \mathrm{~g}$. $\left.\mathrm{ml}^{-1}\right)$ and $\mathrm{NiSO}_{4} \cdot 6 \mathrm{H}_{20}\left(0.605 \mathrm{~g} \cdot \mathrm{ml}^{-1}\right)$ and $1.00 \mathrm{~cm}$ aqueous solution of "Cation X". (Fig. 22. Peak transmission at $264 m \mu$ was $58.0 \%$ and half band width, $29 \mathrm{~m} \mu_{*}$ ). Transmission at $254 \mathrm{~m} \mu$ was $43.2 \%$. 
Another filter system used for the isolation of the $253.7 \mathrm{~m} \mu$ line was a combination $1.00 \mathrm{~cm}$ aqueous solution of potassium iodide (233) and iodine in series with $1.00 \mathrm{~cm}$ of an aqueous solution of cobalt sulfate and nickel sulfate (.113g.m ${ }^{-1}$ and $.604 \mathrm{~g} \cdot \mathrm{ml}^{-1}$. Peak transmission $253.7 \mathrm{~m} \mu$, was $24.0 \%$, half band width, $15 \mathrm{~m} \mu$ ). Although the emission line at $366 \mathrm{~m} \mu$ would have been transmitted by the former set-up ("Cation X" and $\left.\mathrm{CoSO}_{4}-\mathrm{NiSO}_{4}\right)(4.02 \%)$, the source from which the lines had been isolated had a very low output at $366 \mathrm{~m} \mu$ and thus was virtually excluded. Iines at $313 \mathrm{~m} \mu$ were conveniently absorbed by both systems. A Bausch \& Iamb grating monochromator (\#33-86-07, 1200 grove, $\mathrm{mm}^{-1}$, linear dispersion, $7.4 \mathrm{~m} \mu \mathrm{mm}^{-1}$ ) was used in conjunction with the Orram HBD-200 watt light source for the determination of transmissionreflection ratio of the beam splitters at $254 \mathrm{~m} \mu$ and at $290 \mathrm{~m} \mu$. Half band widths at 254 and $290 \mathrm{~m} \mu$ were $25 \mathrm{~m} \mu$ and $13 \mathrm{~m} \mu$, respectively.

Nickel sulfate hexahydrate ( $\mathrm{N}_{150} \cdot 6 \mathrm{H}_{2} \mathrm{O}$ ) and Cobalt sulfate heptahydrate ( $\mathrm{BDH}$ ), potassium iodide, iodine, (Fisher) and napthalene (B\&A) were used without further purification. "Cation X" or 2,7-Dimethyl-3,6-diaza-1,6diene iodide was prepared by light modification of the procedure given in reference (232c).

Glacial acetic acid, (two $6 \mathrm{ml}$ portions BDH, Analar reagent) was added to a mixture of anhydrous ethylene 


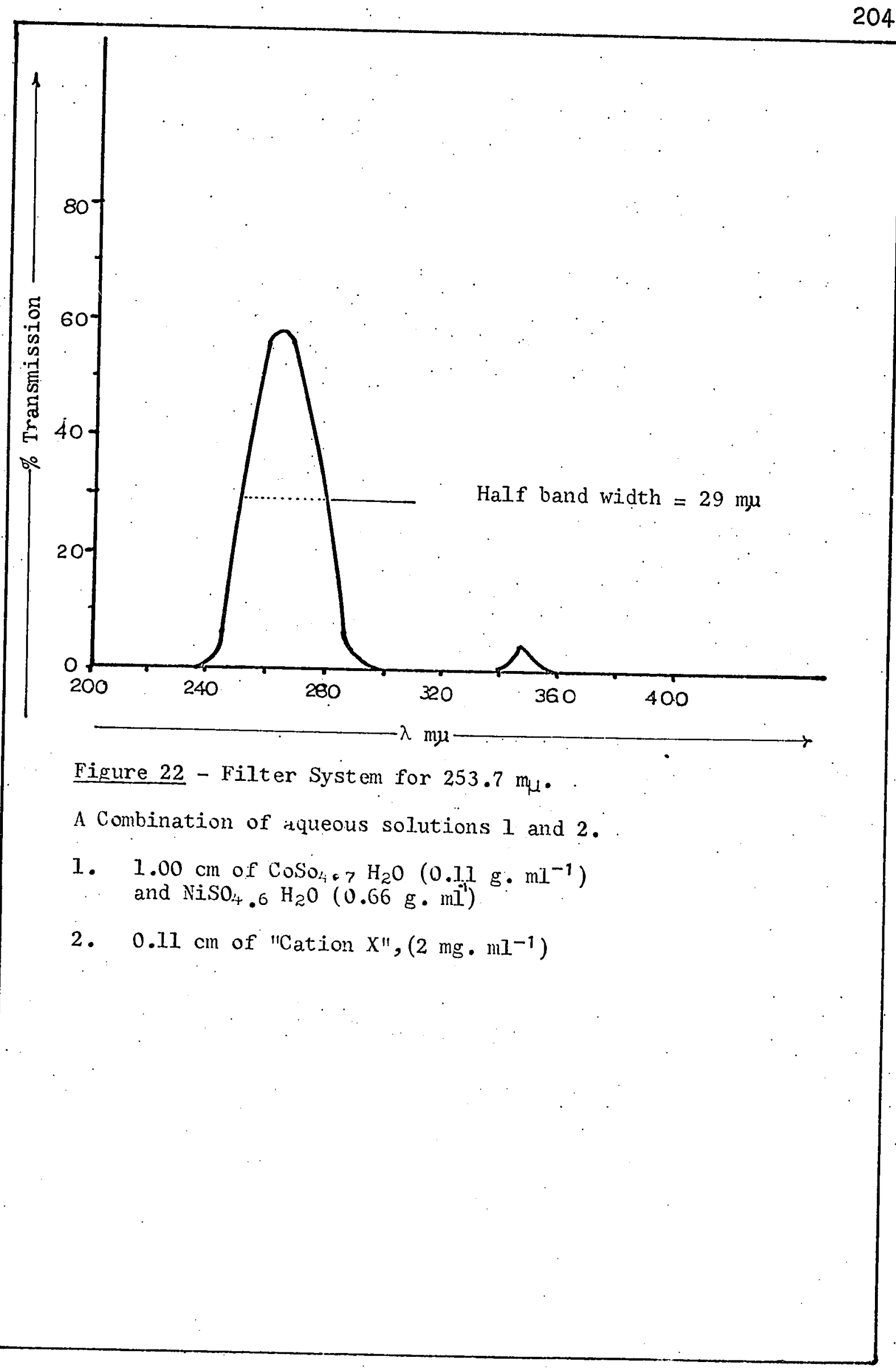




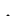

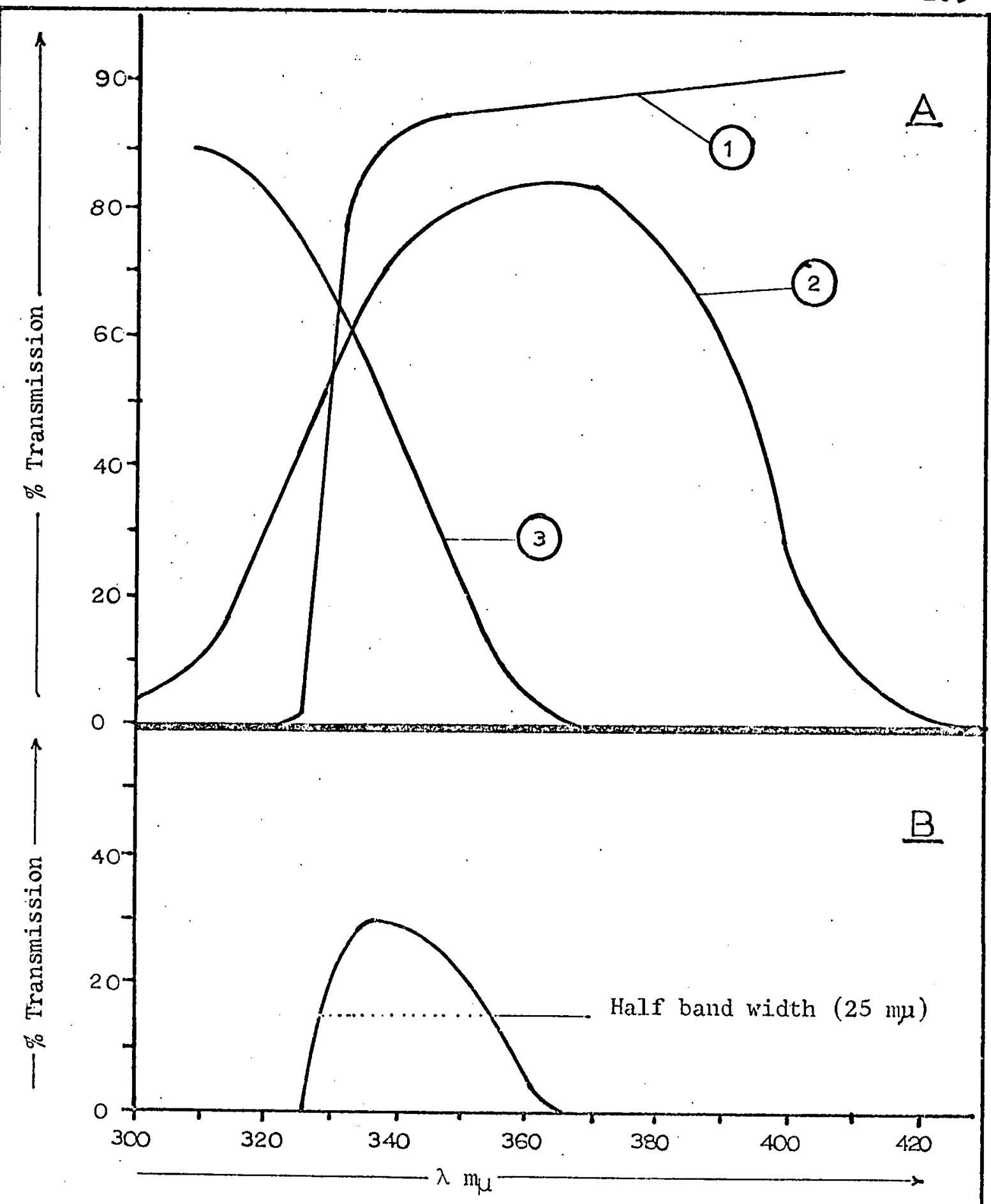

Figure 23 - Filter System for the Isolation of the $334 m_{\mu}$ line from Osrum HBO--200 W. Mercury Lamp.

A. Filter systems 1,2 , and 3

1. $1.00 \mathrm{~cm}$ of naphthalene in cyclohexane (saturated)

2. Corning Glass absorption filter $\mathrm{C} 7-51$

3. $1.00 \mathrm{~cm}$ of aqueous solution of $\mathrm{NiSO}_{4} \cdot 6 \mathrm{H}_{2} \mathrm{O}\left(0.50 \mathrm{~g} \cdot \mathrm{ml}^{-1}\right)$

B. A combination of filters 1,2 and 3. (Calculated \% Transmission) 
diamine (2ml, Fisher reagent) and acetylacetone (2ml, Fisher reagent) maintained at a temperature of $120-130^{\circ}$, and the reaction mixture was heated for 10 minutes at $120-130^{\circ}$. After cooling, the entire content of the flask was poured into ice cold water ( $40 \mathrm{ml}$ ), and $42 \%$ hydroiodic acid ( $8 \mathrm{mI}$ ) was added. Water and excess reagents were removed 'in vacuo' and the resulting residue was taken up in the minimum volume of boiling water. "Cation $X "$, which crystallized out on cooling, was filtered off and recrystallized from ethanol (II4mgs) (mp.243.5-244.5 ${ }^{\circ}$. The U.V. spectrum of this salt was in good agreement with that reported in the literature (232a)* Comparison of the transmission of the salt preparea by this procedure is given at various wavelengths. (conc. $m g \cdot \mathrm{ml}^{-1}$, in water).

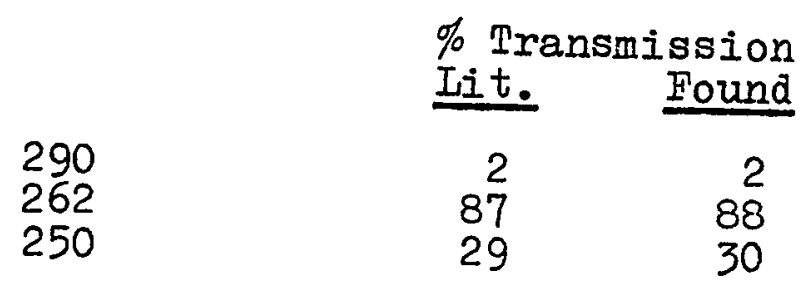

IV. Actinometry

The intensity of the filtered light transmitted by the beam splitter was monitored by chemical actinometry. The choice of the actinometer solution was the ferrioxalate actinometer of Hatchard and Parker (234). Before any quantum yields of benzamides at $210 \mathrm{~m} \mu$ and $254 \mathrm{~m} \mu, 290 \mathrm{~m} \mu$ and $334 \mathrm{~m} \mu$ were determined, the transmission-reflection ratio $(T / R)$ of the beam splitter at specified wavelengths was

*In the literature, the ultraviolet spectrum of "Cation X" has been reported but no detailed method of preparation or its physical constants have been given. 
determined by measuring the amount of light reflected and transmitted over the same period of time. The respective ratios of the beam splitter (G.E. C/P I0I, 4" $\times 4^{\prime \prime} \times 1 / 8^{\prime \prime}$ ) were $9.84 \pm 0.43,8.64 \pm 0.30$ and $9.41 \pm 0.23$ at $210 \mathrm{~m} \mu$, $254 \mathrm{~m} \mu$ and $290 \mathrm{~m} \mu$. The respective half-band widths were $10 \mathrm{~m} \mu$, $25 \mathrm{~m} \mu$, and $13 \mathrm{~m} \mu$. The monochromatic beam isolated by the Bausch \& Lamb grating monochromator was set at an appropriate slit-width for determination of the $T / R$ ratios of the latter two wavelengths. The ratio $(T / R$ ) of the beam splitter (Esco Product, 3" $x$ 3" $\times 1 / 16 "$ "Type GI) was $10.3 \pm 0.30$ at $290 \mathrm{m \mu}$, and the half band width was $25 \mathrm{~m} \mu$. 'the light was filtered through a Baird Atomic Filter. The $T / R$ ratio beam splitter at $334 \mathrm{~m} \mu$ was assumed to be 10 . The intensity of the monochromatic beams at the wavelengths pertinent to the studies was about $1 \times 10^{15}$ quanta $\mathrm{sec}^{-1}$. Quantum yields for ferrous ion production at $210,254,290$ and 334mwere taken as 1.28*, $1.28,1.24$, and 1.24 , respectively. The tjme of exposure was chosen so that the actinometer solution continued to absorb 99.9\% of the light beam throughout the duration of the irradiation. For a long period of exposure (20-24 hrs.) one $10.00 \mathrm{ml}$ aliquot, or two $5.00 \mathrm{mI}$ portions of the solution were used, thereby ensuring that no bleaching of the actinometer solution occurred.

The split-beam irradiation system was chosen for the determination of the quantum yield of benzamide because any small changes in the intensity of the light beam during the *The value determined in our laboratory was 1.4 . 
irradiation (20-24 hrs.) could be experienced by both the actinometer and the photolyte (235). The irradiation beam splitter and cell holders were enclosed in a box painted black and covered by two layers of thick black cloth in order to prevent any interference from stray light. During the irradiation period, a slow stream of nitrogen "scrubbed" through the Fieser's solution (236, ref.225, p.299) was bubbled through the actinometer solution in order to mix the solution.* Any change in the production of ferrous ion due to light absorption was readily measured from a previously determined calibration curve.

Manipulation of the actinometer solution was conducted in a dark room provided with a safe light (Kodak Safe light, Type OA). The output from this lamp did not affect the actinometer solution significantly even after the exposure to the light over a period of two hours. A calibration curve is given in Fig. 24 .

Potassium ferrioxalate hexahydrate $\left(\mathrm{K}_{3} \mathrm{Fe}\left(\mathrm{C}_{2} \mathrm{O}_{4}\right)_{3} \cdot 6 \mathrm{H}_{2} \mathrm{O}\right.$ was prepared from ferric chloride and potassium oxalate (Fisher, reagent) (234). The first crop of crystals obtained was recrystallized three times in the dark room under light from Kodak safe lamp No.OA and dried in a current of air from a hair aryer.

\footnotetext{
* For irradiations lasting longer than 20 hours, the nitrogen was initially bubbled through a column of water and then into the actinometer solution, thereby occurred during the exposure to light.
} 
For calibration, ferrous sulfate $\left(\mathrm{FeSO}_{4} \cdot 7 \mathrm{H}_{2} \mathrm{O}, 6.945 \mathrm{gm}\right)$, * was dissolved in 250ml of $0.1 \mathrm{~N}$ sulfuric acid solution, and diluted to $1 / 250$ of the initial concentration to give a standard $\left(1 \times 10^{-4} \mathrm{M}\right)$ solution. Optical densities of the solution containing various amounts of ferrous sulfate and a constant amount of $0.1 \mathrm{~N} \mathrm{H}_{2} \mathrm{SO}_{4}$, buffer solution, and $0.1 \%$. 9,10 phenanthridine were measured at 510m $\mu$ with a HitashiPerkin Elmer Ultraviolet Spectrophotometer Model 139 and a calibration curve was obtained by plotting the optical density against the concentration of ferrous ion.

C. Cross-Over Experiment with Iabelled Benzaldoxime-180 I. Preparation of 180 -labelled Potassium hydroxylamine disulfonate

Isotopically enriched potassium nitrite was prepared by equilibration between potassium nitrite $(8.5 \mathrm{mg})$ and isotopically enriched water (12.5mI, 1.82 atom percent, 180 ) acidified to a pH of approximately 4 with concentrated nitric acid $(237,238)$. After the equilibrating solution had been allowed to stand for four days, the water was pumped off, the resulting residual salt taken up in normal water (12.5mI), and the content of the flask was pumped down to dryness again 'in vacuo'. The residue was dissolved in isotopically enriched water (12.5mI), acidified to a $\mathrm{pH}$ of approximately 4 ,

* It was unnecessary to determine the purity of the salt against a standard solution because the calibration curve obtained by omitting the standardization procedure was virtually identical. Both U.V. spectrophotometers, Cary 14 and Hitashi-Perkin Elmer-139, could be used interchangeably since there was no difference in their
reading of the absorbance. 


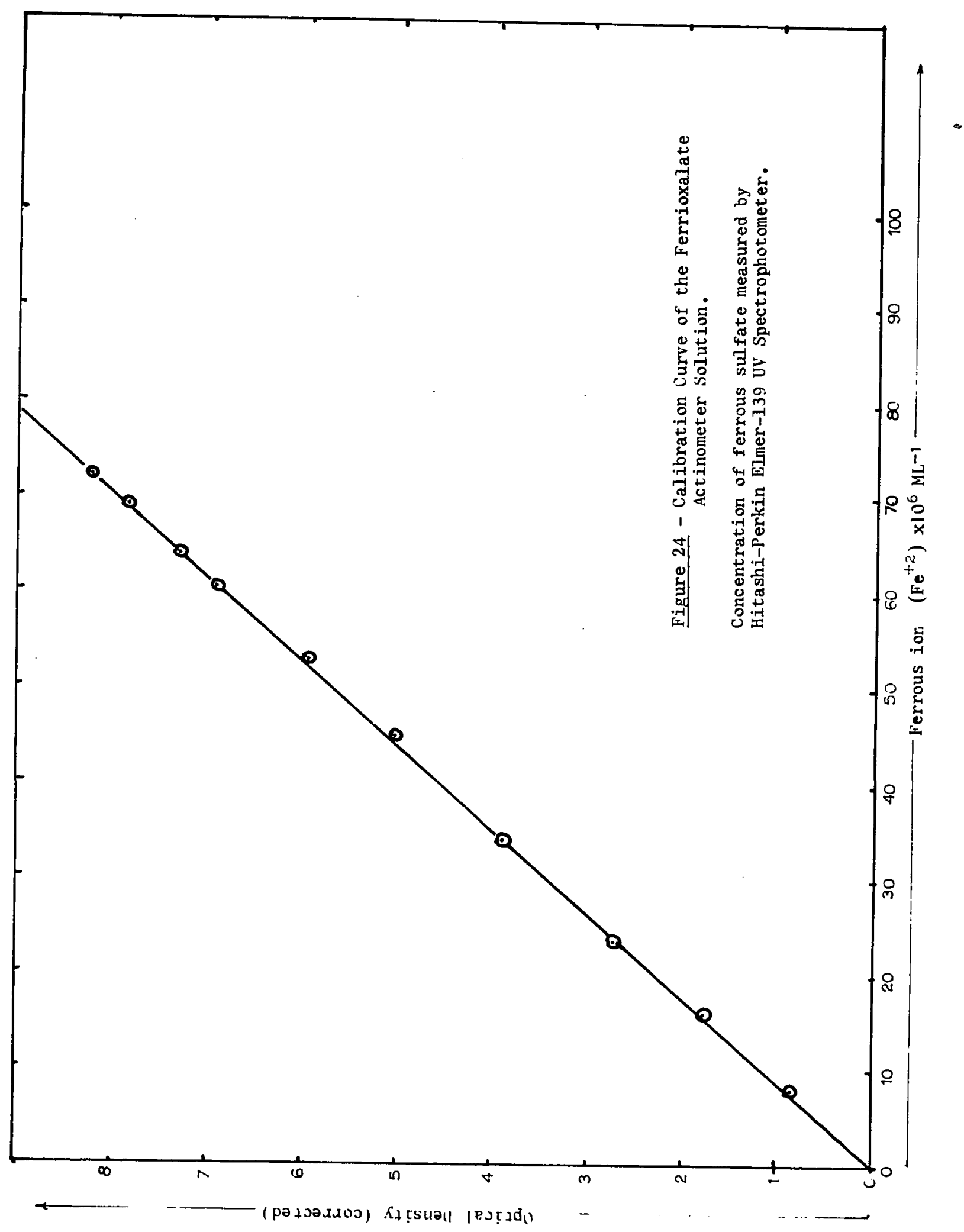


and the reaction mixture was allowed to stand overnight again to attain equilibrium. The solution was diluted with enriched water (62mI), cooled with a dry: 1ce acetone bath, and then saturated with sulfur dioxide gas while maintaining the temperature of the reaction mixture below $\theta^{\circ}$ (239). The Potassium hydroxylamine disulfonate which was precipitated was filtered, washed several times with ice-cold normal water using a 5ml. portion each time, and dried 'in vacuo'. The yield was $19.62 \mathrm{gm}(89 \%)$.

II. Hydrolysis of ${ }^{18} 0$-labelled Potassium Hydroxylamine Disulfonate to Hydroxylamine. $18_{0}$

Potassium hydroxylamine disulfonate (13.44gm) was hydrolyzed in $0.5 \mathrm{~N}$ hydrochloric acid ( $62 \mathrm{ml}$ ) by maintaining the reaction mixture at $100^{\circ}$ for two hours. Upon cooling, barium chloride dihydrate $(60 \mathrm{gm})$ was added and the reaction mixture was stirred vigorously. The contents of the flask were evaporated to dryness 'in vacuo', and hydroxylamine hydrochloride (3.86g) in the residue was extracted with absolute ethanol.

III. Preparation* of Benzaldoxime- ${ }^{18} 0$ A mixture of pyridine ( $14 \mathrm{ml}$ ), ethanol (40ml) and benzalahyde $(5.93 \mathrm{~g})$ was added to the labelled hydroxylamine hydrochloride $(3.86 \mathrm{~g})$ and the resulting reaction mixture was stirred vigorously with a magnetic stirring bar overnight (241). The excess solvent and the pyridine was evaporated

* Reactions of IN-phenylhydroxylamine 180 have been recentIy reported by Nieman and co-worker (99), but no method of isotopic synthesis accompanied their poper. 
off 'in vacuo' and then the residue was taken up in a weak alkaline aqueous solution of sodium carbonate and exhaustively extracted with ethyl ether. After drying the extracts over anhydrous sodium sulfate, the ethyl ether was distilled off and the residue was distilled under reduced pressure on an air bath $\left(94-95^{\circ} / 4 \mathrm{~mm}\right)$.

Because the distillate contained a mixture of benzaldoxime and a small amount of a carbonyl compound, the latter was conveniently removed by filtering an ethereal solution of the distillate through a thin layer of alumina. Upon evaporation of the excess ethyl ether and subsequent distillation, benzaldoxime 180 was readily identified by comparison of the infra-red spectrum with that of an authentic sample and was sent for 180-analysis to the Analytical Corporation. (Found $180,1.54$ atom percent excess; Natural abundance of 180 is 0.20 atom percent).

IV. Irradiation of a Mixture of Benzalcioxime. ${ }^{18} \mathrm{O}$ and p-Tolualdoxime- ${ }^{16} \mathrm{u}$

Equal molar amounts $(0.00414 \mathrm{M})$ of benzaldoxime- ${ }^{18_{0}}$ ( 1.54 atom percent excess) and normal p-tolualdoxime, dissolved in glacial acetic acid (200ml), were placed in an immersion well and irradiated through quartz with a G.E. 85 watt medium pressure mercury lamp. During the irradiation, a slow stream of nitrogen "scrubbed" with a Fieser solution was bubbled through the solution. After eight hours, irradiation was 
discontinued and the solvent was evaporated off 'in vacuo', while the temperature of the distilling flask was kept below $35^{\circ} \mathrm{C}$. The brown residue ( $1.53 \mathrm{gm}$ ) was taken up in chloroform and filtered through a sintered glass filter to remove suspended insoluble material.

Separation of benzamide from p-toluamide was readily effected by vapour phase chromatography (vpc) on an Aerograph gas chromatographic instrument distributed by Wilkens Instruments \& Research, Inc., using a $51 \times \frac{1}{4} "$ column packed with 3\% Craig (butanediol succinate) and $1 \%$ carbowax $20 \mathrm{M}$ on 60-80 mesh chromosorb $W$ supplied by Wilkens Instrument \& Research, Inc. The following experimental conditions were employed: Column temperature, $190^{\circ}$; helium gas flow rate I70m I min. ${ }^{-1}$; retention time of benzamide, 21 min.; retention time of p-toluamide, $35 \mathrm{~min}$. Both the benzamide and p-toluamide fractions were collected and crystallized from benzene and sent for analysis to Analytical Corporation, N.Y. Mp of benzamide was (125.5-126.5 , Iit. 125-126, 130 $)$ and that of p-toluamide was $\left(157-158^{\circ}\right.$, Iit. $\left.159-160^{\circ}, 165^{\circ}\right)$. Mixedmelting points of these amides with authentic samples were not depressed.

Before samples were sent for analysis, small amounts of both amides were rechromatographed and the results clearly indicated that neither was contaminated by the other. 
Found: Benzamide 180 ( 1.54 atom percent excess); p-toluamide $18_{0}$ (0.034 atom percent excess).

In order to achieve a proper separation of the two amides by the method employed (VPC), it is advisable to utilize a new column which had been properly conditioned for preparative purposes. Although an $85 \%$ recovery of amides can be readily effected, the constant use of the column for one month rendered it incapable of efficient separation of these two amides.

D. Irradiation of $\alpha-p$-Anisaldoxime at Low Temperature I. Optical Absorption Cell

The cell consisted of an all-quartz Dewar flask with four optically polished plane parallel windows. The solution was contained in an optical quartz cell, ( $40 \times 10 \times 10 \mathrm{~mm})$ fused by means of a graded seal to a pyrex No. B-24 ground glass cone, which in turn was attached to a B-10 ground glass cone suitable for attachment of a stop-cock unit connected to a vacuum line. This latter modification was necessary for degassing the solution before irradiation at low temperature. The temperature of the cell was measured with an Iron- Constantan thermocouple connected directly to a recorder (Sargent SR). The cell was cooled by introduction of precooled nitrogen gas into the Dewar through one of the inlets. A detailed description of the cell is given by Hirshberg and Fisher (100). 
A stream of nitrogen was flushed through a column (Im $x$ 40mm) of anhydrous silica gel, bubbled through liquid nitrogen, and then passed through a copper coil immersed in a very large Dewar flask (2I Iitre capacity) filled with liquid nitrogen. The temperature of the cell could be adequately controlled by regulating the flow of the coolant by means of a fine needle valve (Nupro), as long as water was prevented from clogging the line.

Stray light which might have interfered with the absorption measurement was eliminated by thoroughly wrapping the exterior part of the quarts Dewar with some black friction tape; this avoided any elaborate technique for housing the cells. The entrance of stray light at the junction of the monochromometer and detector unit and the quartz window of the Dewar was similarly prevented. Periodic absorption measurements were taken on the irradiated solution merely by blocking off the incident light. Optical density measurement was monitored by a direct connection of the output from the detector to a recorder (Sargeant SRI).

\section{Iight Source}

An Osram HBO-200 watt high pressure mercury lamp was used throughout the experiment. The mercury emission lines were isolated with a Baird Atomic Interference filter at $290 \mathrm{~m} \mu$. Irradiations of the solutions were carried out at right angles to the measuring beam. The filtered light was 
directed perpendicularly to the Original path from the source by using a quartz prism.

\section{Irradiation in a Fluid Modium and U.V.} Spectrorhotometry: General Procedure

The general set-up of apparatus and instruments is schematically presented in Fig. 1. The low-temperature technique employed was essentially that described by Hirshberg and Fischer (100) withafer minor modifications. Rather than the use of Beckmann $D$ or Cary Model 14, a single beam ultrav10let spectrophotometer (Hitashi-Perkin Elmer-139) was adopted for measurement of optical absorption of a solution over a temperature range $\left(-90\right.$ to $\left.+25^{\circ} \mathrm{C}\right)$. $4.0 \mathrm{ml}$ of solution of $\propto$-p-an1saldoxime dissolved in $n$-hexane $\left(7-8 \times 10^{-5} \mathrm{ML}^{-1}\right)$ were degassed at a pressure of $0.1 \mathrm{~mm} \mathrm{Hg}$, and 1rradiated at $290 \mathrm{mp}$ through parallel windows with a diameter of $1.00 \mathrm{~m}$. The optical density of the solution was measured periodically between $211 \mathrm{mp}$ and $350 \mathrm{m \mu}$ at a f1xed sl1t w1dth $(0.95 \mathrm{~mm})$. Irradiation of the solution was confined to the temperature range, $-90^{\circ} \mathrm{C}$ to $+25^{\circ} \mathrm{C}$. Because the instrument was a singlebeam spectrophotometer, a blank of the pure solvent, taken at room temperature, was assumed to be essentlally the same at all temperatures. An appreclable correction has not been found to be necessary for hydrocarbons (100). The output of light from the hydrogen lamp incorporated into the spectrophotometer unit was extremely stable for several hours: A drift no greater than $\$ .003$ absorbance unit in one hour 
could be observed when measurements were made by both the meter and the recorder. A comparison of a U.V. spectrum taken at room temperature by this particular arrangement of apparatus was made with that taken on another Cary 14 spectrophotometer and the spectra were superimposeable, indicating the reliability of the measurements. Changes in the spectra on 1rradiation and alteration of temperature were readily measured.

Several 1rradiated samples $\left(-53^{\circ}\right)$ were combined, concentrated 'in vacuo' and analyzed by ILC, eluting with ethyl ether - petroleum ether $(5: 1 \nabla / v)$.

Eacause the solution appeared fairly mobile at temperatures as low as $-80^{\circ}$, several solutions were 1rradiated with a slow stream of nitrogen, providing some mixing. A capillary tube was used for this purpose and was removed each time spectral measurements were being taken.

\section{Irradiation in a Rigid Matrix at $77^{\circ} \mathrm{K}$}

1. General Procedure:

In this experiment, a quartz cyclindrical cell (O.D. = $15.4 \mathrm{~mm}$ ) fitted with a tapered B-10 ground glass cone was used. The oxime solutions were made up in MCP and degassed at 0.005 $\mathrm{mm}$ by the "freeze-thaw-freeze" method. The cells were $1 \mathrm{mmersed}$ in Ilquid nitrogen in a phosphorescence Dewar flask and 
irradiated through the transparent quartz windows at the bottom of the Dewar flask. The light beam from a HBO-200 watt high pressure mercury lamp was focused on the solid solution with a quartz lens. During the irradiation the cell was rotated through $360^{\circ}$ in order to give even exposure to light on all sides of the cell. In certain instances the cell was removed from the liquid nitrogen bath and rapidly immersed in an ethanol slush $\left(-117^{\circ}\right)$ and shaken vigorously to mix the solution.

2. Reaction of Photolyte with Phenyl Isocyanate: When the irradiation at $77^{\circ} \mathrm{K}$ had been discontinued, $250 \mu l$ of phenyl isocyanate were injected into the cell with a syringe bearing a 6" needle. The mouth of the cell was quickly capped to minimize the entry of moisture. The cell was allowed to warm to $-117^{\circ} \mathrm{C}$, and was agitated to disperse phenyl isocyanate throughout the solution.

Both the solvent and the excess phenyl isocyanate were removed 'in vacuo' and finally dried for several hours under vacuum $(0.2 \mathrm{~mm})$, while the cell was refrigerated at $-60^{\circ}$ in dry ice-acetone bath. The contents of the cell were allowed to warm to $0^{\circ} \mathrm{C}$ and were then taken up in a suitable solvent for infra-red analysis or for thin layer chromatography. Recovered solutions which had been used to take the infra-red spectra were also applied to thin layer plates coated with silica gel (0.025" thick). Invariably, an unexposed sample 
of the same volume of solution as the photolyte was treated in an analogous manner to serve as a control.

$3.0 \mathrm{mI}$ of o-anisaldoxime $\left(0.017 \mathrm{~m}^{-1-1}\right)$ were irradiated at $77^{\circ} \mathrm{K}$ for 20-30 hours with filtered light of wavelength $290 \mathrm{~m} \mu$. The solvent and excess phenyl isocyanate were removed in vacuo' and the residue was analyzed by thin layer chromatog raphy as described above. p-Anisamide and p-methoxyformanilide, were separated on the plate with (ethyl etherPetroleum ether $\left.35-60^{\circ}, 5: 1\right)$, scraped off into a $10.0 \mathrm{ml}$ volumetric flask, extracted with cyclohexane-n-propanol, $(4 / 1)$, centrifuged, and analyzed spectrophotometrically to estimate the yield. The molar extinction coefficients of $p$ anisamide at $252 \mathrm{~m} \mu$ was $€=14,800$, and that of p-methoxyformanilide at $251 \mathrm{~m} \mu$ was $€=13,600$. Irradiated solutions which were not treated with phenyl isocyanate were worked up in a similar way. Where the identity of the amides was in doubt, they were recovered from the solution used for U.V. spectral analysis by "freeze-drying" under a pressure $2.0 \mathrm{~mm}$ of $\mathrm{Hg}$, taken up in chloroform, and injected into a V.P.C. column. The The chromotagraph was an Aerograph HY-FY (Wilkens)-Model 600C; column 15' $\mathrm{x}$ I/8" packed with chromosorb W (80 mesh) and coated with Craig (3\%) and Carbowax 20M (1\%); the column temperature was $204-205^{\circ}$ and the pressure was 40psi. The instrument was capable of detecting up to $1 \times 10^{-4} \mathrm{mg}$ of these amides. Comparisons of the retention times of the amides photochemically produced were made with those of authentic 
samples. The retention times of p-methoxyformanilide and anisamide were respectively,.19mins. and 34mins. from the chloroform peak.

3. Reaction of Photolyte with Potassium Iodide: $3.0 \mathrm{ml}$ of saturated potassium iodide in isopropanol were added to an irradiated solution of $\propto$-p-anisaldoxime which had been irradiated at $290 \mathrm{~m} \mu$ for $30 \mathrm{hrs.}$; a control sample containing an equal volume of solution unexposed to light was also treated in the same way, and the colour change was observed. The residue after removal of the solvent was applied to a TIC plate and eluted with petroleum-ether (30-60) and ethyl ether $(4: I)$.

E. Separation of $\alpha$ and B-Benxaldoximes by Thin Iayer Chromatography: Quantitative Analyses

I. Method I: Measurement of Spot Area (103) Smooth glass plates (8" $x$ 8" $x 1 / 8 "$ ) were coated with a $0.25 \mathrm{~mm}$ layer of fluorescent Kieselgel or Silicagel $\mathrm{CF}_{254}$ (Herskag) $\left(20 \mathrm{gm}+40 \mathrm{ml}\left(\mathrm{H}_{20}\right)+5 \mathrm{ml}(\mathrm{MeOH})\right.$ by means of a Research Specialties thin layer applicator. The plates were well dried in the oven at approximately $110^{\circ}$, allowed to cool, and then used immediately for thin layer chromatography. Spots of a $0.5 \%$ ethanolic solution of a mixture of $\alpha-$ and $\beta-$ benzaldoxime $\left(2-3 \times 10^{-3} \mathrm{mg} \mu^{-1}\right)$ were applied from an Aloe micro pipette $(6 \mu \mathrm{l})$. The development was carried out by the ascending method in a rectangular tank and the adsorbates were eluted until the solvent front (Petroleum ether $35-60^{\circ}$, 
ethyl ether, 4:I) had almost traversed the entire length of the plate. For adequate equilibration and good development, the two broad sides of the tank were well lined with appropriate size pieces of filter paper. The developed chromatograms were removed from the tank and dried; the spots of $\propto$ and $\beta$-benzaldoximes which separated out were detected readily under an ultraviolet lamp. A record of the area of the spots was made by tracing the spots on a piece of tracing paper held over the glass plate on the side free of the silica gel. It was necessary to shine the ultraviolet light directly on the side coated with the silica gel and to make a trace of the developed spots on the opposite side since the glass plate was opaque to ultraviolet light. Alternatively, a record of the area of the developed spots was made by tracing the area of the spots directly on the glass using a felt pen soaked with black ink and making a Xerox copy of the trace. The $R_{f}$ value of $q$-benzaldoxime was 0.30 and that of $\beta$-benzaldoxime was 0.15 .

II. Method II: Measurement by Direct U.V. Spectrophotometry

Smooth glass plates (8" $x$ 4" $x \mathrm{z} / 8$ ") were coated with a $0.75 \mathrm{~mm}$ or layer of fluorescent Kieselgel (20 gm $+40 \mathrm{mI}\left(\mathrm{H}_{20}\right)$ - 5 ml $\left(\mathrm{M}_{\mathrm{eOH}}\right)$ ) by means of a CAMAG-Model-A thin layer applicator. The plates were thoroughly dried in an oven at a tempezature of $110-120^{\circ}$ allowed to cool and stored in a 
moisture-free chamber. Spots of a $2.4 \%$ solution (20pl spots of almost equal amounts of $\alpha-$ and $\beta$-benzaldoximes $2 \times 10^{-30} \mathrm{mg}$

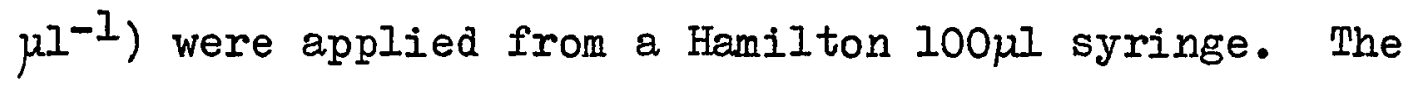
development of the chromatogram was carried out by eluting the plate upward with a 4:1 mixture of petroleum-ether (BDH, Analar, distilled in a spinning band column, (35-60\%), ) and

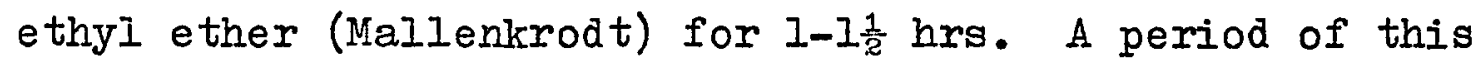
duration was required for a good separation because the amount spotted on the plates was quite large. The developed chromatograms were removed from the tank and partially aried, and the spots of $\alpha$ - and $\beta$-benzaldoximes were readily detected under the ultraviolet light. The spots were scraped off the plates into a $50 \mathrm{ml}$ volumetric flask with a razor blade. After making up the solution to its appropriate volume with a 4:1 mixture of cyclohexane and n-propanol, the oximes were extracted from the silica gel by vigorously shaking the flask and allowing it to stand for at least two hours. The solutions of a- and $\beta$-oximes were centrifuged in order to precipitate out the suspended silica gel and the amounts of $\alpha-$ and $\beta$-oximes were determined spectrophotometrically on a Bausch and Lamb 505 or a Cary-Model-14 U.V. spectrophotometer. The optical quartz cell used in these measurements had a p.I. of $1.00 \mathrm{~cm}$.

The amounts of oximes present in the solution were readily calculated based upon the pre-determined molar extinction coefficients of the $\alpha-$ and $\beta$-oximes. The 
extinction coefficients $\left(\epsilon_{\alpha}\right.$ and $e_{\beta}$ ) of $\alpha$ and $\beta$-1somers in 4:1 cjclohexane-n-propanol are $\epsilon_{255 \mathrm{mp}}=14,300$ and $\epsilon_{248 \mathrm{mu}}=$ 14,300 , respect17ely. $\lambda_{\max .}$ are quoted here.

This particular solvent system was sultable for the quantitative estimation of these oximes by the spectroscopic method because it was capable not only of extracting both isomeric forms, but was also incapable of suspending silica gel to any appreciable extent, methanol in contrast gave a considerable interference due to suspension of silica gel. Cyciohexane could not be used at all because 1t extracted only 31\% of the oximes. Solvent combinations such as the ethanol-cyclohexane, and ethanol-cyclohexane proved to be impractical owing to the low miscibility of the alcohols with cyclohexane.

III. Extraction of $\alpha$-and $B$-Benzaldoximes from the Silica Gel Using a 4:1 Crclohexane-n-Propanol Solvent System

The efficiency of $\alpha$ and $\beta$-benzaldoxime recovery was established by the following method.

A standard volume ( $100 \mu I)$ containing a known amount of of-benzaldoxime and $\beta$-benzaldoxime, dissolved in ethanol, (95\%), was applied with a Hamilton 100 ul micro syringe as a band ( $4 \mathrm{~cm} \mathrm{Iong)} \mathrm{to} \mathrm{a} \mathrm{plate} \mathrm{coated} \mathrm{with} \mathrm{silica} \mathrm{gel} 10.75 \mathrm{~mm}$ thick). After the solvent had evaporated off, an area of 
silica gel $(2 \mathrm{~cm} \times 5 \mathrm{~cm})$ was scraped off into a $(50.00+.05 \mathrm{ml})$ volumetric flask with the aid of a funnel, a 4:I mixture of cyclohexane and n-propanol was added to the flask and made up to the appropriate volume. The solution was allowed to stand at room temperature for two hours and was shaken intermittently during this period. Approximately 10ml of the solution were centrifuged to remove the small amount of the suspended silica gel. The clear supernatant liquid was then analyzed spectrophotometrically by a Cary 14 U.V. spectrophotometer.

A comparison with another series of unspotted samples which served as a control was made in order to determine the loss that might have occurred on the silica gel by employment of this particular method.

\section{F. Sensitized Reaction}

I. A Protonic Reaction Conditions in Dioxane

The quantum yield of benzamide was measured under conditions where the sensitizers were capable of transferring triplet energy to the oximes. B-Benzaldoxime (0.05 - .08M) and a sensitizer $(0.013-0.016 \mathrm{M}$, triphenylene, phenthrene, or anthracene) were dissolved in dioxane and irradiated with light emitted from an Osram HBO-200 watt mercury lamp filtered through $1.00 \mathrm{~cm}$ of a saturated solution of naphithalene in cyclohexane. Iines transmitted were at wavelengths above 325mp. To estimate the amount of light absorbed by the sen- 
sitizers, the flux at $334 \mathrm{mp}$ was chosen since the filter was opaque to all the emission lines below 325m $\mu$, and the sensitizer was transparent to lines above $360 \mathrm{m \mu}$. Moreover, lines above $325 \mathrm{~m} \mu$ were not absorbed by either oxime. Inspection of the spectral distribution of the Osram-HBO-200 watt lamp clearly indicated that the only major emission line between $325 \mathrm{~m} \mu$ and $360 \mathrm{~m} \mu$ is that at $334.2 \mathrm{~m} \mu$. To estimate the intensity of the light of this wavelength, the flux was first determined by a combination of filters $(1.00 \mathrm{~cm}$ saturated solution of napthalene in cyclohexane; $1.00 \mathrm{~cm}$ solution $\mathrm{N}_{i} \mathrm{SO}_{4}-6 \mathrm{H}_{2} \mathrm{O}$; (0.5gm.m.-1; 5mm Corning filter C-7-5I); and then the amount of light which would have been transmitted without the two filters was calculated from the transmission of the beam at $334 \mathrm{~m} \mu$; this was $35.7 \%$. Thus the flux estimated in this way could be applied to the calculation of the total amount of light at $334 \mathrm{~m} \mu$ which would have been absorbed by the sensitizers. The concentration of the sensitizers was chosen in such a way that they absorbed geater than $99 \%$ of the light at $334 \mathrm{~m} \mu$ during the entire irradiation.

During the irradiation conducted in the presence of triphenylene, a stream of nitrogen "scrubbed" by the Fieser solution was slowly bubbled through the photolyte. After the irradiation was discontinued, aliquots of $5.00 \mathrm{ml}$ were taken out and the solvent was sublimed off under a pressure of 2-3mm of $\mathrm{Hg}$ in a dessicator. For the infra-red spectrometric 
analysis the "blank" sample containing the appropriate amount of $\alpha-$ and $\beta$-oximes and triphenylene was prepared after the photostationary ratio of $\alpha$ - and $\beta$-benzaldorime had been determined. A similar "blank" was also prepared for phenanthrene. The flux transmltted through the quartz beam splitter at $334 \mathrm{mp}$ was measured by the ferrous oxalate actinometer-beam splitter system and the amount incident on the irradiation cell was found to be approximately $5.8 \times 10^{-19} \mathrm{q}$ $\mathrm{hr} .^{-1}$ ( 3 determinations). Irradiation in the presence of phenanthrene was conducted in a $5.00 \mathrm{ml}$ irradiation cell, whlch had been provided with a degassing unit. The conventional "freeze-pump-thaw" operation was emplojed. The flux of $4.1 \times 10^{-19} \mathrm{q} \mathrm{hr} .^{-1}$ was similarly estimated at $334 \mathrm{mp}$ ( 5 determinations). Anthracene was also used as a sensitizer but no attempt was made to detect the presence of benzamide in the photolyte. The only results recorded were the observations of $\alpha \beta \beta$ 1somerization detected by TLC.

The quantum jield of benzamide which had been irradiated in neat acetone is also included since the absorption of light at $290 \mathrm{mp}$ was essentially that by the acetone rather than by the oxime. The intensity of the light was monitored in the usual manner on the reflectance side of the beam splitter.

In order to establish that energy transfer from the sensitizer to the exime could be readily effected in the 
presence of acetic acid, the change in the relative amounts of $\alpha$-benzaldoxime and B-benzaldoximes was followed by TIC analysis.

II. Protonic Reaction Conditions in Cyclohexane The quantum yield of benzamide was determined at $334 \mathrm{~m} \mu$ in the presence of 100 equivalents of acetic acid and triphenylene dissolved in cyclohexane. The flux at this wavelength was measured on the reflected side of the beam splitter (Esco, GI, 3" x 3" x I/IO") by the ferrous oxalate actinometer during the irradiation of the substrates using the filter systems described above. $5.00 \mathrm{ml}$ of photolyte contained in a reaction cell equipped with a degassing unit were used in the irradiation of benzaldoximes. The flux at $334 \mathrm{~m} \mu$ was estimated as $1.8 \times 10^{-19} \mathrm{hr} .^{-1}$ (3 determinations). Two samples were irradiated under these reaction conditions with light filtered through $1.00 \mathrm{~cm}$ of a saturated solution of naphthalene in cyclohexane. The formation of substances other than benzamide which could contribute to the carbonyl absorption at $1676 \mathrm{~cm}^{-1}$ in the I.R. spectrum was excluded by analyzing irradiated solutions which contained only acetic acid and triphenylene, and benzaldoxime and acetic acid. These "controls" were subjected to reaction conditions identical to those employed in the presence of triphenylene.

The amount of benzamide which had formed in the presence of acetic acid (100 equivalents) was estimated by infra-red 
spectrophotometry; and the amounts of 0.1 and $0.15 \mathrm{mg}$ were determined from two separated runs. Solutions which had been subjected to infra-red analysis were recovered, concentrated 'in vacuo' and subsequently analyzed by TIC on a 0.025 " layer of silica gel. The tile was eluted with a 4:I mixture of cyclohexane and ethyl ether, and developed under ultraviolet light. A fluorescent band coincident with that from an authentic sample of benzamide spotted on the same plate was scraped off into a volumetric flask (10.00), made up to the appropriate volume, and extracted with a $4: I$ mixture of cyclohexane and n-propanol. The suspended silica gel was conveniently removed by centrifuging and the supernatant liquid was analyzed by ultraviolet spectrophotometry, using a Perkin-Elmex-Hitashi-I39 and a Bausch and Iemb-505 spectrophotometer.

The reliability of the TLC-Spectrophotometric method for the estimation of benzamide was tested by determining the uncertainty in the amount that could be extracted from the TIC plate coated with thick silica gel $0.75 \mathrm{~mm}$. This uncertainty was $0.01 \mathrm{mg}$. Essentially, 90\% recovery could be achieved if the amount of benzamide applied to the plate was about $0.1 \mathrm{mg}$.

The identity of benzamide was also confirmed by VPC. The column, (10' $\times 1 / 81$ ) was packed with 80 mesh chromsorb-W, coated with Craig (3\%) and Carbowax-20M, (1\%); it operated at $172-173^{\circ} \mathrm{C}$ under $40 \mathrm{psi}$ of helium and was used for the detection 
of benzamide. The limit of berizamide detected was determined on the Areograph HY-FY, equipped with a flame ionization detector operating under the conditions already cited. The retention time of benzamide, operating under these conditions, was 21.3 minutes from the ethyl acetate peak. Neither triphenylene nor B-benzaldoxime interfered with the benzamide chromatographic peak. The retention time of triphenylene was 120 minutes. Further, it had been previously shown, in connection with another aspect of the problem that $\beta$-bendaldoxime had a retention time which was not atiall similar to that of benzamide on the same column. The limit of visual detection of benzamide on the TIC tile was $2 \times 10^{-3} \mathrm{mg}$ and by VPC was also $2 \times 10^{-3} \mathrm{mg}$. The only substance which could have interfered with the VPC detection of benzamide were either $\alpha-$ benzaldoxime, or its decomposition products, or thermally isomerized substances, since injection of a small amount of $\alpha$-benzaldoxime generated at peak coincident with that of benzamide. Therefore, under the conditions employed for VPC analysis, the limit to the amount of the $\alpha$-isomer required before the interfering peak became noticeable on the chromatogram was determined as $61 \times 10^{-3} \mathrm{mg}$ far in excess of the detectable amount of benzamide $\left(2 \times 10^{-3} \mathrm{mg}\right)$.

In general, benzamide was characterized in the following manner:a) A carbonyl band at $1676 \mathrm{~cm}^{-1}$ was in agreement with that of an authentic sample, b) Analyses by TLC-Spectrophotometry and gas chromatography (gave supporting evidence, since 
the developed chromatograms showed a spot whose position corresponded to that of an authentic sample of benzamide which had been spotted on the same plate. Extraction of the spot from the silica gel followed by UV analysis showed a well defined spectrum of benzamide. The yield of the benzamide could also be estimated by the latter method based on the previously determined molar extinction coefficient $(9,460)$ at $226 \mathrm{~m} \mu$ of benzamide in $4: 1$ cyclohexane and $n$-propanol. Furthermore, the residue recovered from the sample prepared for UV spectrophotometry was analyzed by VPC using an Aerograph HY-FY apparatus equipped with a flame ionization detector. The VPC trace showed a peak with the same retention time as benzamide. Neither triphenylene nor $\beta$ benzaldoxime interfered with the analysis. A method of analysis using the combination of TLC, UV, and VPC enabled one to calculate the limit of the interfering amount of $\alpha-$ benzaldoxime that might have contributed to the benzamide peak in the VPC chromatogram in the event of separation by TLC being incomplete. The limit was readily estimated by assuming that the absorption at $258 \mathrm{~m} \mu$ in the UV spectrum was due to $\propto$-benzaldoxime; the estimate would then be in excess of the actual amount of the oxime. TIC separation of small amounts of photolyte used in the experimental determination of quantum yields was usually efficient. Interference by $\propto-$ benzaldoxime or triphenylene in the UV or VPC analyses was negligible. 
III. "Prep Scale" Sensitized Reaction of Benzaldoxime under Protonic Conditions in Cyclohexane

An apparatus was constructed for the purpose of preparing benzamide by sensitization with triphenylene in the presence of acetic acid (100 eq) in cyclohexane. The top portion of an Analar acetic acid bottle (80 fluid oz.) was cut to the length of $25 \mathrm{~cm}$. Then an immersion well was filled with a saturated solution of naphthalene in cyclohexane in order to provide a filtering system for the isolation of the $334 \mathrm{~m} \mu$ Iine emitted from a high pressure mercury lamp (450 watt). Triphenylene ( $1.6 \mathrm{gm})$, acetic acid (25mI), and $\alpha$-benzaldoxime $(0.50 \mathrm{gm})$ were dissolved in $1500 \mathrm{ml}$ of cyclohexane which had been passed through a column of alumina and degassed with nitrogen for $1 / 2 \mathrm{hr}$. The transmission of the solution at $334 \mathrm{~m} \mu$ was $0.1 \%$, due to the absorption by triphenylene. The addition of acetic acid or a-benzaldoxime did not affect the transmission at this wavelength.

During the irradiation, the solution turned yellow despite the passage of nitrogen through it. After the irradiation was terminated (24 hrs.), the solvent was evaporated off and cyclohexane and methanol were added to the resulting residue to crystallize out some of the triphenylene. Triphenylene was filtered out and the filtrate was concentrated 'in vacuo'. The residue was separated by TIC on a .025" layer of silica gel, eluted with ethyl ether-cyclohexane $(4: I)$ and subsequently developed under ultraviolet 
light. The first chromatograph did separate the bulk of the triphenylene from the benzamide, but a complete separation was not accomplished. This was clearly evident in the ultraviolet spectrum of the extracted band having the same $R_{f}$ value as an authentic cample of benzamide. Even after the second chromatograph, the presence of triphenylene was noticeable, although the purity of the extract had increased. A shoulder in the UV spectrum due to the benzamide at $228 \mathrm{~m} \mu$ was, however, apparent. Even after the third chromatograph, complete removal of triphenylene was not effected. However, the identity of the henzamide was verified by VPC.

\section{G. Quantum Yield of $\alpha$-Benzaldoxime $\left(\phi_{B \alpha \alpha}\right)$ and B-Benzaldoxime $\left(\phi_{\mathrm{QB}}\right)$ at $290 \mathrm{m \mu}$ in $\mathrm{n}$-Hexane, Cyclohexane and Dioxane}

The photochemical efficiencies of syn-anti, or cistrans or $\alpha-\beta$-isomerization of benzaldoximes were determined in n-hexane (BDH, Spectroscopic), cyclohexane, and dioxane. This quantum yield of $\beta$-benzaldoxime from $\alpha$-benzaldoxime ( $\left.\phi_{\phi \beta}\right)$ was measured in the three solvents and the reverse process $\left(\phi_{\beta \alpha}\right)$ was determined only in dioxane at $290 \mathrm{~m} \mu$ between $23-28^{\circ} \mathrm{C}$.

\section{In n-Hexane}

The concentration of $\propto-$ benzaldoxime in $n$-hexare was made up to absorb $99 \%$ of the light at $290 \mathrm{~m} \mu(28.4 \mathrm{mg} / 25.00 \mathrm{ml}$ or $0.0094 \mathrm{~N} ;)$. Three consecutive samples $(5.00 \mathrm{mI})$ were 
cooled in dry ice-acetone, degassed at a pressure of $0.005 \mathrm{~mm}$ of $\mathrm{Hg}$, allowed to warm to room temperature, cooled to $-57^{\circ} \mathrm{C}$, and degassed again. This process of degassing was repeated three times for each sample. Then samples were irradiated for 40 minutes. The intensity of the light was monitored with $5.00 \mathrm{~m}$ (1.00 cm.p.I.) of the ferrioxalate actinometer solution and the entire content was diluted to $25.0 \mathrm{ml}$ for development.

The amount of $\beta$-benzaldoxime formed upon irradiation was assayed by the application of the TIC-Direct spectrophotometric method. The entire content of the photolyzed solution was spotted on the $0.75 \mathrm{~mm}$ silica gel plate. During the application of the photolyte to the plate a slow stream of cold air from a hair dryer was directed at the origin. $\propto$ and $\beta$-Benzaldoximes were very readily separated with petroleumether $\left(35-60^{\circ}\right)$ and etry ether $(4: 1, V / V)$ as long as the application of the band at the origin was confined to within $1 \mathrm{~cm}$. The band corresponding to $\beta$-benzaldoxime which was developed under ultraviolet light was scraped off into a volumetric flask, and extracted with a solvent mixture of $4: I$ cyclohexane and n-propanol $(50.0 \mathrm{ml})$. The solution was allowed to stand for two hours and then about $10 . \mathrm{ml}$ was centrifuged and subsequently analyzed spectrophotometrically on the recording Cary-14 U.V. Spectrophotometer.

In order to correct for any thermal conversion of $\mathbf{a}$ to $\beta$-benzaldoxime that might have occurred during the irradiation 
an equal volume of an unexposed solution was spotted on the same plate.

Although the unexposed solution did not usually show any visible amount of $\beta$-isomer, nevertheless, the region which would have corresponded to $\beta$-oxime was scraped off and worked up in the usual manner. The difference in absorbance between the irradiated and unexposed samples was taken as the amount of $\beta$-benzaldoxime photochemically produced. The difference in optical density determined represented about 6.5-7\% conversion from $\alpha-$ to $\beta$-benzaldoxime.

Although the volume applied to the plate was enormous, because of the facile separation of $\alpha$ and $\beta$-benzaldoxime on the plate, care in spotting a narrow band at the origin was unnecessary.

An attempt to irradiate $\beta$-benzaldoxime in a solution of identical concentration was not entirely feasible due to its insolubility in $n$-hexane. To dissolve an amount of $\beta$-isomer equal to that of $\propto$-isomer slight application of heat was required. This in turn demanded a large correction for the thermal isomerization of the thermodynamically less stable $\beta$-isomer to the $\alpha$-isomer.

The temperature of the housing was measured with a IronConstantin thermocouple coupled to a recorder (SargentRecorder, model SR) with a reference temperature of $0^{\circ}$ 
(ice bath).

\section{In Dioxane}

Quantum yields of $\alpha$-benzaldoxime ( $\left.\phi_{\alpha}\right)$ and $\beta$-benzaldoxime $\left(\phi_{\alpha \beta}\right)$ in dioxane at $290 \mathrm{~m} \mu$. were determined by the same method. Both $\alpha$-and $\beta$-benzaldoximes were dissolved in dioxane $(0.22 \mathrm{Im} \cdot)$, and irradiated at $290 \mathrm{~m} \mu$ for 2 hours. The beam was adjusted to fill a rectangular ultraviolet quartz cell (capacity $300 \mu \mathrm{l}, \mathrm{p} .1 .1 .00 \mathrm{~mm}$ ) provided with a Teflon stopper. Because the cell was not fitted with adequate outlets for degassing, the solvent was passed through a column of alumina and degassed for one hour by bubbling a stream of nitrogen which had been "scrubbed" by Fieser's solution. The effect of the inadequate degassing technique was tested by irradiating a solution through which oxygen, rather than nitrogen, had been bubbled for one hour. The entire content of the photolyzed sample (300pl) was applied directly to the silica gel plate and eluted with petroleum-ether $\left(35-60^{\circ}\right)$ and ethyl ether $(4: I, V / V)$. The band applied at the origin was $5 \mathrm{~m} \times 40 \mathrm{~mm}$, and was suitable for separation on the plate. To the same plate used for chromatographing an irradiated solution, an equal volume of unexposed solution was also spotted and similarly worked up. Yields of the oximes produced photochemically were determined spectrophotometrically, in the manner already described for determination of $\phi_{q \beta}$ in n-hexane. 
$5.00 \mathrm{ml}$ of ferrioxalate actinometer solution was used to monitor the intensity at $290 \mathrm{~m} \mu$ and the entire content was diluted to $25.0 \mathrm{ml}$.

\section{In Cyclohexane}

$5.00 \mathrm{ml}$ of $q$-Benzaldoxime dissolved in cyclohexane (8-9 $\mathrm{x}$ $10^{-3} \mathrm{MI}^{-1}$ ) were irradiated for approximately 1 hour at $290 \mathrm{~m} \mu$. During the irradiation a slow stream of nitrogen was bubbled through the solution. After the irradiating beam had been turned off, equal volumes of both irradiated and unexposed solutions of $\alpha$-benzaldoxime were diluted to $(25.00 \pm .03) \mathrm{ml}$ in a volumetric flask, and the solutions were diluted once more by pipetting $0.80 \mathrm{ml}$ into a $25.0 \mathrm{ml}$ volumetric flask. The change in the absorbance due to irradiation was measured at $260 \mathrm{~m} \mu$ with a single-beam, manually operated. U.V.-Spectrophotometer (Hitashi-Perkin-Elmer-139) at a fixed slit width and the amount of $\beta$-oxime formed photochemically was determined by application of the Difference Method. This method first involved the determination of the molar extinction coefficients of $\alpha$ - and $\beta$-benzaldoximes at various wavelengths, and then a selection of suitable wavelengths at which there was a large difference in the extinction coefficients. In this experiment, $260 \mathrm{~m} \mu$ was selected since it provided a large difference between the two extinction coefficients $\left.\Delta e_{\alpha \beta}=\epsilon_{\alpha}-\epsilon_{B}=5350\right)$. Although the spectra of these two oximes do not have large differences in their extinction coefficients in the region where the spectra are flat, the 
260mp region of spectra was quite appropriate because the two spectra are almost parallel here and this reduced possible error that might have occurred in reproducing the same wavelength. Because $\delta e_{\alpha \beta}$ was required, a region in the spectra at $260 \mathrm{~m} \mu$ where the two were parallel would thus make $\Delta \epsilon_{\mathrm{d} \beta}$ independent of $\lambda_{i}$.

For 9-10\% conversion, a difference of -0.024 in absorbance unit was measured.

The intensity of the light was measured in the usual manner with $5.00 \mathrm{ml}$ of the ferrioxalate solution. The entire content of the solution was developed for measurement.

\section{Analytical Procedures}

1. MLC-Direct U.V. Spectrophotometry:

In $n$-hexane, the quantum yield of $\beta$-benzaldoxime $\left(\phi_{\alpha \beta}\right)$ was $283+0.002$. The application of TLC-Direct Spectrophotometry method was very reliable and gave readily reproducible results. Possible errors due to the instrument itself were reduced by measuring an absorbance difference of approximately 1.

Attempts to measure the quantum yield $\left(\phi_{\beta \alpha}\right)$ under identical conditions were discontinued when the $\beta$-isomer failed to dissolve in n-hexane to a concentration comparable to that used for the irradiation of the $\alpha$-isomer. Although 
B-benzaldoxime could be dissolved by warming the solution, a large blank correction due to thermal isomerization made the results somewhat unreliable.

Separation of $\beta$-isomer from the $\alpha$-isomer by TLC was excellent. No apparent formation of benzamide or other side products under the experimental consitions employed were detectable on the TIC tile. Even if benzamide had been formed, it would have been readily removed by chromatography since, under the conditions of elution of the tile, benzamide would have remained at the base line.

The excellent resolving power, rapidity, and simplicity of thin layer chromatography made it attractive for the analysis of both $\alpha$-benzaldoxime and $\beta$-benzaldoxime mainly because these two isomers are heat sensitive (decomposes to benzonitile, benzaldehyde, and probably benzamide) for gas chromatographic techniques, which otherwise might have been a suitable alternative method for analysis.. Similar decomposition of benzaldoxime has been reported by Lohr and Warren (101), and thermal decomposition of simple aliphatic oximes on the column has been also reported by Cason and Harris (102).

Two methods employing TLC for quantitative analyses were attempted. The first method (103) involving the measurement of spot area without the removal of the oximes from the thin 
layer plate did not give reproducible results. Although the separation of the two benzaldoximes were excellent, (eluting with a solvent mixture of petroleum ether (35-60) and ethyl ether $(4: I, \nabla / v)$ and the shapes of the developed spots on the chromatograms were uniformly circular; this method was abandoned.

The second method was TIC-Spectrophotometry, for quantitative analysis of both $\alpha$ - and $\beta$-benzaldoximes. This involved the extraction of the oximes from the absorbent, followed by direct spectrophotometric measurement on a recording U.V. spectrophotometer. Quantitative, reproducible results were obtained by this method which offered the important advantage that the spectra of the oximes recovered from the plates were superimposable on those of unspotted materials, thus enabling one to scan the entire spectrum for possible contamination of the solution. The accessible region for most suitable measurement was $225 \mathrm{mp}$ to $350 \mathrm{m \mu}$ of the U.V. spectrum. Petroleum ether $\left(35-60^{\circ}\right)$ and ethyl ether $(4: I, V / v)$ were used for eluting the plates because they had the significant advantage that they were not only extremely volatile and hence readily removed from the plates after the development of the chromatogram, but in addition, they did not interfere with the spectrophotometric measurements. Another useful solvent system was a $4: I(\nabla / v)$ mixture of cjclohexane and $n$-propanol, which was extremely suitable for spectrophotometric measurements in the ultraviolet region. 
Unlike polar solvents such as ethanol and methanol (104) which required large "blank" corrections in the 225-350m $\mu$ region of the ultraviolet spectrum owing to the suspension of an appreciable amount of silica gel, the use of cyclohexanen-propanol mixture did not. Usually, a correction of only -0.001 absorbanc unit was required for the blank using a cell of $1.00 \mathrm{~cm} \mathrm{p.I.} \mathrm{between} 240 \mathrm{m \mu}$ and $260 \mathrm{mp}$. Only occasionally was a correction of: +0.014 absorbance unit required, after the plates had aged for about a week. In solvents such as methanol, a large correction was required even in the visible region of the spectrum. Inconsistancies reported by other workers employing this TLC-Spectrophotometric method (104) may be due to the gradual deposition of a film of silica gel on the windows of the cell after a series of successive measurements using the same cell.

The most important property of the solvent system chosen for UV spectral measurements is its capacity to extract both $\alpha$-and -benzaldoximes to the extent of $99 \%$ from the absorbent. The desired substance was therefore essentially recoverable from the plates in its pure form, as indicated by the spectral identity of the spotted and the unspotted samples. Extraction of substances from silica gel with an efficiency as high as $97.5 \%$ by Bird (105) has been reported.

Both $\alpha$-and $\beta$-benzaldoximes were stable in cyclohexane and n-propanol (4:1) over a period of 30 hours at room temperature. 
Neither the intensity nor the position on the absorption maxima underwent any observable change over this period. Similar stability of these two isomers on silica gel has been reported by Hranisavljevic-Jakovljevic and co-workers (106).

\section{The Difference Method (107):}

When the concentration of only one absorbing constituent of a mixture of two components is sought, it is often possible to determine the total absorption of the mixture at a suitable wavelength, then to remove the constituent in question, then to redetemine the absorption of the residual material. The difference between the two absorptions therefore is a function of the substance removed. In this instance where B-benzaldoxime was the particular constituent sought for in a mixture of both $\alpha$ - and $\beta$-benzaldoximes, the Difference method was applied to spectrophotometric analysis.

Absorbance measurements were made in the region of $260 \mathrm{~m} \mu$ where the spectra of both $\alpha$ - and $\beta$-isomers are almost parallel. This selection reduced the possible errors arising from reproducing the same wavelength at $260 \mathrm{mp}$. The concentration of B-benzaldoxime was calculated using equation 49. Equation 49 is derived in the following way.

Assuming that the Beer-Lambert Law is obeyed at all wavelengths one can write the total absorbance due to $\alpha$ - and $\beta$-isomers at a particular wavelength $\left(\lambda_{i}\right)$ as 
Eq. $46 \quad(O D)_{\lambda_{i}}^{t}=\left(\epsilon_{\alpha} \cdot\left[\alpha_{t}\right]+\epsilon_{\beta} \cdot\left[\beta_{t}\right]\right)_{i}$

The absorbance of the unexposed solution $(O D)_{\lambda_{i}}^{0}$ containing only the $\alpha$-isomer is given by

Eq. 47

$$
\begin{aligned}
& (\infty)_{\lambda_{i}}^{0}=\left(\epsilon_{\alpha}\right)_{i} \cdot\left[\alpha_{0}\right], \text { where }[\alpha]=\left[\alpha_{0}\right] \text { and } \\
& {[\beta]=0}
\end{aligned}
$$

After irradiation, isomerization from $\alpha \rightarrow \beta$ occurs and the final concentration, $\left[\alpha_{t}\right]$ of $\alpha$ will be

Eq. $48 \quad\left[\alpha_{t}\right]=\left(\left[\begin{array}{ll}\alpha & 0\end{array}\right]-\left[\beta_{t}\right]_{t}\right.$

The combination of equations 1,2 , and 3 gives

Eq. $49\left[\beta_{t}\right]=\frac{(O D)_{\lambda_{i}}^{t}-(O D)_{\lambda i}^{0}}{\left(\epsilon_{\beta}-e_{\alpha}\right)}+\frac{\Delta(O D)_{\lambda_{i}}}{\Delta\left(e_{\alpha \beta} \lambda_{i}\right.}$

$\epsilon_{\alpha}$ and $\epsilon_{\beta}$ are respectively defined as the extinction coefficients of $\alpha$ and $\beta$-benzaldoximes, $(O D)_{\lambda_{i}}$ as the absorbance or the optical density at a particular wavelength, $\left(\lambda_{i}\right)$ and $[\alpha]$ and $[\beta]$ as the respective concentrations of $\alpha$ and $\beta$-benzaldoximes.

From this equation the yield, and hence the quantum yields can be determined.

The application of the Difference method to the estimation of $\beta$-benzaldoxime assumes that the Beer-Lambert Law is obeyed at all the concentrations. In fact, a series of optical measurements at various concentrations indicated that this assumption was valid. It also assumes that the 
total reduction in the concentration of the two isomers within the period of exposure undergoes no appreciable change in that interference by rearranged products (benzamide) with the absorbance measurement is negligible. Because the efficiency with which $\alpha$ or $\beta$-benzaldoxime isomerizes is far greater than that of benzamide formation in cyclohexane, both the reduction of the isomers and the contribution of benzamide. to the absorption were negligible. The molar extinction coefficient of benzamide at $260 \mathrm{~m} \mu$ in cyclohexane is only 580 compared with $\epsilon_{Q}$ of 11,000 and $e_{\beta}$ of 5670 for $\alpha$-benzaldoxime and $\beta$-benzaldoxime, respectively.

Four successive measurements of the quantum yield $\left(\phi_{\alpha \beta}\right)$ determined by this method gave $\phi_{\alpha \beta}=0.30 \neq 0.02$, indicating the reproducibility of the results. The values obtained in n-hexane by the TLC-U.V. Spectrophotometric method was 0.283 . These two values determined by both methods are in good agreement with each other.

H. Cis-Trans Isomerization: Photostationary States I. Direct Irradiation

All direct irradiations of the oximes were carried out on the optical bench except for that at $253.7 \mathrm{~m} \mu$. For the source of the $253.7 \mathrm{~m} \mu$ line $(96 \%)$ a low pressure mercury vapour lamp mounted circularly in the Rayonet Photochemical Reactor RPR-100 were used to irradiate a solution of oximes (0.02M) contained in a quartz tube. A quartz water jacket 
surrounding the tube maintained the temperature of the reaction at $22^{\circ}$. By circulating some cold water from the tap through a coil of copper tubing which had been immersed into the quartz water jacket surrounding the irradiation tube, the temperature of the reaction medium could be conveniently regulated to $22^{\circ}$. Aliquats from the degassed solution were taken out periodically and analyzed the TLC-U.V. Spectrophotometric method.

The relative amounts of $\alpha$ and $\beta$-benzaldoximes were determined by taking aliquots periodically during the irradiation and separating by TIC. The chromatogram was developed by eluting with cyclohexane-ethyl ether. The two geometric forms were readily detected under the ultraviolet light and were scraped off into a $50.00 \mathrm{ml}$ volumetric flask, allowed to stand for 4-24 hrs., and then the amounts of the two forms were measured spectrophotometrically with a Bausch and Lamb 505 spectrophotometer. The relative amounts of the $\beta$ and $\alpha$ forms could be readily computed from their predetermined molar extinction coefficients in the $1: 4 \mathrm{n}-\mathrm{PrOH}-$ cyclohexane mixture. Both $\epsilon_{\alpha}$ and $\sigma_{p}$ at 255 and $248 \mathrm{~m} \mu$ were 14,300. The results of the photochemical isomerization are given in the Table 5. Graphs (Fig. 6) of the ratio(B/o1)were plotted against time in order to determine the photostationary states under various reaction condtions. 
II. Sensitized Irradiation

A solution of benzaldoximes contained in a quartz cell (1.00 cm path length, 0.D. 40mm, capacity $18.6 \mathrm{ml}$ ) was irradiated using an Osram HBO 200 watt high pressure, mercury vapour lamp. The power was supplied from a Bausch \& Lamb SP200 or from a Gates P-210-D power supply. The entire optical system was placed on an optical bench and the beam from the lamp was filtered through a $(1.00 \mathrm{~cm})$ layer of water and was passed

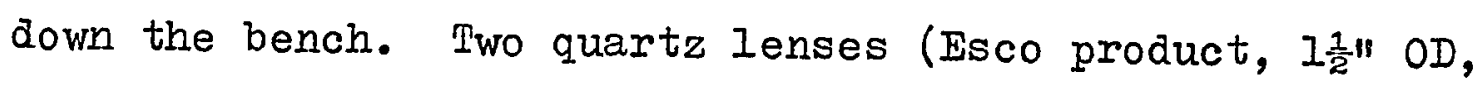
f.I.=3") were used to collimate the light. Appropriate filter systems were utilized during the investigation of sensitized cis-trans isomerization of the benzaldoximes. For reactions conducted in the presence of triphenylene and phenanthrene, $1.00 \mathrm{~cm}$ of a saturated solution of naphthalene in cyclohexane was suitable for filtering the lines to which benzaldoximes are transparent (325mu). All irradiations were performed at room temperature.

I. Emission Spectrophotometry Quenching of Phosphorescence of Sensitizers by $\alpha$-and $\beta$-Benzaldoximes at $77^{\circ} \mathrm{K}$ and at $22^{\circ} \mathrm{C}$

I. Materials

Benzophenone and triphenylene were used as donor sensitizers for experiments at $77^{\circ} \mathrm{K}$, and biacetyl was selected at $22^{\circ} \mathrm{C}$. EPA was selected as a suitable solvent for recording of phosphorescence spectra. 


\section{Instrumental and Procedure}

A Jarrel-Ash Ebert Scanning spectrometer (Model \#82-029, f.1.500mm) blazed for $5000 \mathrm{~A}^{\circ}$ was used to record the phosphorescence spectra of solutions prepared for emission studies. The detector unit consisted of a Universal Photometer, model No. 201, equipped with a photomultiplier tube (RCA Type 93IA, S-4 response; peak sensitivity at 4000A. The recorder used to monitor the output of spectral response was a Leeds-Northrup, model Speedomax-G, with a response time of one second, 0-I MV full scale response, and a chart speed of $2 " /$ minute. This corresponds to $9.6 \mathrm{mu} / \mathrm{minute}$.

The light source was a Hanovia D.C.Xenon Arc Iamp (D-901C) used in conjunction with a Bausch and Lamb grating monochromator (model, \#33-88-07; reciprocal linear dispersion, $74 \mathrm{~A} / \mathrm{mm})$. The light emitted from the exit slit set at $3.4 \mathrm{~mm}$ was passed down the optical bench and reflected at right angles to the incident radiation by a front surface plane mirror ( $5 \mathrm{~m} \times 5 \mathrm{~m} \times$. $^{\prime \prime}$ ), aluminized and over-coated for ultraviolet irradiation. The reflected light was then focused on the irradiation cell with a quartz lens $(f .1 .=10 \mathrm{~cm})$. The scanning spectrometer was set up to measure the emission along a direction at $90^{\circ}$ to that of the radiation incident on the cell and hence vertical cyclindrical quartz cells were used. Emission light from the irradiation cell was focused on the entrance slit of the scanning spectrometer. 
diameter of the quartz cell was $6 \mathrm{~mm}$ (I.D.) and for measurement at $22^{\circ} \mathrm{C}$, the inside diameter was $26 \mathrm{~mm}$ (I.D.). These cells were fitted with a quartz-pyrex graded seal, a combination tapered B-10 joints grease trap, and a lmm bore vacuum stop cock connected to a vacuum line for degassing the solutions at $77^{\circ} \mathrm{K}$ by the "freeze-pump-thaw" method at $0.003 \mathrm{~mm}$ of mercury. Apiezon-I grease was used on all the ground glass joints because of its low vapour pressure.

The phosphorescence measurement on benzophenone and triphenylene were made with compounds dissolved in rigid glass at $77^{\circ} \mathrm{K}$. A phosphoroscope rotating can was used to isolate phosphorescence from the source emission and sample fluorescence. For measurements of emission from biacetyl at $22^{\circ} \mathrm{K}$ in a fluid medium, the phosphoroscope rotating can was removed and the total emission of the solution was recorded. The exciting line selected for benzophenone was $365 \mathrm{m \mu}$, for triphenylene, 334mp, and for biacetyl, $425 \mathrm{~m} \mu$. The constancy of the incident light could be monitored by periodically taking several emission spectra of solutions of the same concentration. Only when this could be achieved, were the quenching experiments attempted. Calibration of the chart paper was made by concurrently monitoring the lines from a mercury pen lamp.

In order to record the quenching of phosphorescence by \& and B-benzaldoximes in EPA, a standard solution of the donors (sensitizers) was first prepared and stored in the 
dark until required. The concentration of benzophenone was $0.035 \mathrm{M}$, of triphenylene, 0.01IM, and of biacetyl, $0.064 \mathrm{M}$. Then a comparison of the emission spectra of the standard solution containing only the donor was made with that containing the same amount of donor and, in addition, the acceptor or the quencher, viz. $\alpha$-benzaldoxime or $\beta$-benzaldoxime. In this experiment the concentration of $\alpha$-benzaldoxime in the standard solution of benzophenone was $0.17 \mathrm{M}$, and in a standard solution of biacetyl was $0.099 \mathrm{M}$. When triphenylene was selected as the donor, the spectrum of a standard solution containing both triphenylene and acetic acid (.0074M) was compared with that containing $\beta$-benzaldoxime (.018M) in addition to acetic acid. For each comparative study, spectra were recorded within $\frac{1}{2}$ to $I$ hour.

J. Quantum Yield Measurements of Benzamide at $210,253.7$, and $290 \mathrm{m \mu}$

I. Direct Irradiation

For most of the irradiation studies a solution of approximately $0.007 \mathrm{M}$-benzaldoxime was used. $5.00 \mathrm{ml}$ of solutions in cyclohexane, acetic acid and dioxane were irradiated. At this concentration the photolyte was capable of absorbing more than 99.9\% of the light at 210, 254 and $290 \mathrm{~m} \mu$ for the duration of the irradiation period ( $20 \mathrm{hrs.}$ ) Moreover, the total amount of oxime was a convenient amount to work up after an irradiation since a "blank" or a "dark" reaction sample for infra-red spectral analysis could be 
readily prepared. Greater amounts of oximes (100-120mg) were somewhat cumbersome to work up because the yield of kenzamide from these irradiations was much smaller relative to the amount of oxime, and this made the determination of the yield of benzamide less reliable.

Before each irradiation, the solution was thoroughly degassed by the "freeze-pump-thaw" method. After the apparatus had been degassed, the cell was carefully inverted so that the solution ran into the irradiation reservoir (see Fig. 21). That the absorption of light was over $99.9 \%$ was checked periodically before and after the irradiation, by use of a Perkin-Elmer, Hitashi-139 U.V. Spectrophotometer. The Irradiation cell was not usually sealed off from the degassing reservoir but was kept intact. However, after an irradiation had been terminated, the irradiation cell and the degassing reservoir of the apparatus were broken apart and the photolyte was carefully poured into a $10 . \mathrm{ml}$ round bottom flask. The photolyte was cooled to the temperature of liquid nitrogen bath and the solvent sublimed off in a dessicator at a pressure of 2-3mm of $\mathrm{Hg}$. No external heat was applied. After the solvent had been removed, the residue was left in a dessicator at $2-3 \mathrm{~mm}$. for $10-12 \mathrm{hrs}$. To the residue, 0.50 or 1.00ml of chloroform (Fisher, spectrograde) was added and the solution was subjected to infra-red spectral analysis. From the intensity of the carbonyl absorption of benzamide at $1676 \mathrm{~cm}^{-1}$, the amount of benzamide which had been photochemically 
generated was calculated from a predetermined calibration curve. The infra-red spectra were taken on Beckman IR-7 operating under the following conditions: Slit width ratio of selection and standard was $4 / 1$ or $3 / 1$ at $1667 \mathrm{~cm}^{-1}$; Gain 10X; period 2; Double beam. The infra-red cell was made from polished sodium chloride plates of $1.00 \mathrm{~mm}$ path length. A new calibration curve was drawn up when the lapse of time between analyses was long. A "blank" or "dark reaction" sample was always subjected to the identical experimental conditions.

The quantum yield of benzamide at $210 \mathrm{~m} \mu$ was calculated by taking the quaritum yield of ferrioxalate as 1.28 which is an extrapolated value from the data obtained by Parker and Hatchard $(184,234,242)$. The values at twelve different wavelengths between $254 \mathrm{~m} \mu$ and $570 \mathrm{~m} \mu$ were recorded. The absorption by the actinometer solution at $210 \mathrm{mp}$ appears to be a continuation of the electronic transition band that begins from the visible all the way up to wavelengths below 200mp. A further correction at $210 \mathrm{~m} \mu$ was necessary when the quantum yield was determined in the presence of 100 equivalents of acetic acid, since both benzaldoxime and acetic acid absorbed in this region. The amount of light absorbed by the two substances was determined by taking the area under the absorption contributed by both compounds. Between 200 and $220 \mathrm{~m} \mu$ (at $210 \mathrm{~m} \mu$, the half band width was $10 \mathrm{~m} \mu$ ), the ratio of the area of benzaldoxime absorption to that of acetic acid (100eqs.) was $3.8 / 1$. 
In the determination of the quantum yield at $253.7 \mathrm{~m} \mu$, the ratio of the optical density of benzaldoxime to that of acetic was only necessary since the line isolated at 253.7 mas $_{x}$ essentially pure owing to the type of low pressure mercury lamp employed and the filter systems had adequately removed the mercury emission lines above $253.7 \mathrm{~m} \mu$.

II. An Estimation of Quantum Yield of Potassium Ferrioxalate Actinometer Solution at $210 \mathrm{m \mu}$

Attempts were made to determine the quantum yield of potassium ferrioxalate calibrated against the uranyl oxalate actinometer at $210 \mathrm{~m} \mu$ by the technique of differential absorption spectrophotometry described by Pitts (242).

Identical amounts of the oxidant ceric sulfate were added to the same volume of irradiated and un-irradiated samples of uranyl oxalate solution. The amount of oxidant was added in slight excess so as to measure the concentration of $\mathrm{Ce}^{\mathrm{IV}}$ ion at $320 \mathrm{~m} \mu\left(e=5.42 \times 10^{-4}\right)$. The difference in the residual concentration of $\mathrm{Ce} \mathrm{IV}^{\mathrm{in}}$ the irradiated and unirradiated samples (blank) was determined by comparison of the two solutions by differential absorption spectrophotometry, and this difference was related to the amount of uranyl oxalate photochemically decomposed. From the determination of the decomposition of ferrioxalate solution, according to the ferrioxalate at $210 \mathrm{~m} \mu$ was calculated, taking the value of 0.50 for the quantum yield of uranyl oxalate at 210m $\mu$ (233, $234,244)$. Because of the inconsistancy of results obtained 
by employing Pitts method, his method was abandoned in favour of a different approach. Instead the quantum yield of potasium ferrioxalate at $210 \mathrm{~m} \mu$ was determined using a splitbeam apparatus.

The use of sodium salicylate as a detector of ultraviolet light has recently found wide practical applications (245). At $600-3500 \mathrm{~A}^{\circ}$, the relative quantum yield of fluorescence of this fluorescent material has been found to be independent of wavelengths in this range, and can be used in conjunction with a detecting source for the measurement of light intensity. Another application of this detecting system is presented for the measurement of the quantum yield of potassium ferrioxalate at $210 \mathrm{~m} \mu$.

A split-beam apparatus for irradiation was set up (Fig. 14). The ferrioxalate solution was irradiated with the beam transmitted through the quartz beam splitter. The extent of photolytic decomposition of the ferrioxalate was determined spectrophotometrically with a Perkin-Elmer-Hitashi-139 according to the procedures of Parker and Hatchard (184). The quantum yield of the compound at $250 \mathrm{m \mu}$ was taken as 1.28 . On the reflected side of beam splitter, an iris diaphram (Ealing) was closed down to its smallest aperture, and the intensity of the light was monitored with a photomultiplier tube IP21. The signal from the tube was fed to an Eldorado Electronic photometer Model 201 which in turn was connected 
to Sargent recorder to monitor the constancy of the source.

The relative intensities of the source at $210 \mathrm{~m} \mu$ and 250m $\mu$ were measured by a Spot Galvanometer Type GMI3C, Radiometer (Copenhagen) connected to a RCA-935 phototube with 55 response. The tube was coated with a thin film $\left(\mathrm{cm}^{2}\right)$ of sodium salicylate (2mg of sodium salicylate was dissolved in methanol and applied over the phototube window and dried very slowly).

For the sources of the lines at $210 \mathrm{~m} \mu$ and $250 \mathrm{~m} \mu$, a Bausch and Lamb grating monochromator, ( $1 / 2$ band width $13 m \mu$ ) was used at a fixed slit width to isolate the beam from a Hanovia 150 watt Xenon-mercury vanour compact lamp.

Under conditions where the intensity of a source is constant, the quantum efficiency of a chemical reaction at one wavelength can be determined. The efficiency of the same chemical processes at another wavelength within the spectral range must be known and the intensity-detecting apparatus must be independent of wavelengths.

A relationship which enables one to calculate the quantum yield within regions useful and accessible to organic chemists is presented below. For this work, the quantum yield of potassium ferrioxalate was determined at $210 \mathrm{~m} \mu$ based upon the known efficiency of the reaction at $250 \mathrm{~m} \mu$ 
(233). The method of calculation is given below.

Defining nhv=number of quanta $(q)$

$$
\begin{aligned}
& x_{i}=\begin{array}{l}
\text { deflection of the galvanometer linearly related to } \\
\text { the intensity }
\end{array} \\
& I_{i}=\text { intensity of a source at a specified wavelength }\left(\lambda_{i}\right) \\
& t_{i}=\text { time of exposure of the photolyte }
\end{aligned}
$$

Eq. 50 Then nhv $=I_{i} t_{i}$

Since $I_{i}=C_{i} \cdot X$ where $=C_{i}$ is a constant due to the geometry of detecting system

Eq. $51 \quad n h v=C_{i} \cdot X_{i}=I_{i} t_{i}$

Defining the quantum yield of the reaction as $i$

Eq. $52 \phi_{i}=\frac{\text { Total number of molecules of ferrioxalate }}{\text { Total number of quanta absorbed by the }}$

$$
\frac{\text { photochemically decomposed }}{\text { ferrioxalate solution }}
$$

Eq. $53 \phi_{i}=n_{i} / I_{i} t_{i}$ where $n_{i}=$ the total number of molecules photochemically decomposed

Eq. $54 \quad \phi_{i}=n_{i} / C_{i} X_{i} t_{i}$

Eq. 55 Therefore $\phi_{1}=\phi_{j}\left(n_{1} \cdot x_{j} \cdot t_{1}\right) / n_{1} \cdot x_{1} \cdot t_{1}$

Leting $\phi_{i}=\phi_{210 m \mu}$ and $\phi_{j}=\phi_{250 m \mu}$

Eq. 56 Then $\phi_{210 m \mu}=\phi_{250 m \mu} \cdot n_{210} \cdot x_{250} \cdot t_{250} / n_{250} \cdot x_{210} \cdot t_{210}$

If the amount of ferrioxalate photochemically de-

composed is determined spectrophotometrically, then

$n_{i} \infty O D_{i} i . e$. to the amount of the change of

ferrioxalate in a given volume.

Eq. $57 \quad n_{i}=$ Cst $\cdot O D_{i}$

Cst is another constant and $O D_{i}$ is optical density at a specified wavelength $\lambda_{i}$ 
Eq. $58 \quad \therefore \phi_{210}=\phi_{250} \frac{(O D)_{210}}{(O D)_{250}} \cdot \frac{x_{250} \cdot t_{250}}{x_{210} \cdot t_{210}}$

Choosing time of exposure so that $t_{250}=t_{210}$

Eq. 59

$$
\phi_{210}=\phi_{250} \times \frac{(O D)_{210} \times x_{250}}{(O D)_{250} \times 210}
$$

The terms on the r.h.s. are all measureable or known quantities.

The data are recorded in Table XII and the quantum yield of ferrioxalate at $210 \mathrm{~m} \mu$ obtained by this method is given in Table XIII. The extrapolated value obtained from the data of Parker and Hatchard was 1.28 .

Although the yield of benzamide could also have been determined by VPC, by calibration with a standard solution containing p-toluamide as an internal standard, this method of analysis was abandoned since a-benzaldoxime decomposed on the column to afford a substance with the same retention time as benzamide under conditions suitable for determination of benzamide. The amount of "benzamide" arising from thermal rearrangement on the column was in the order of $2-3 \%$ and rendered this method of analysis unreliable for the determination of benzamide. However the use of this method for quantitative estimation of benzamide by VPC, is recommended. The VPC chromatograph instrument was Aerograph Hy-FY, Model 6000. From the plot of the ratio ( $X$ ) of the weight (mg) of benzamide to the weight $(\mathrm{mg})$ of $\mathrm{p}$-toluamide $\mathrm{vs}$. the ratio $(Y)$ of the area (arbitrary units) of the curve due to benzamide 

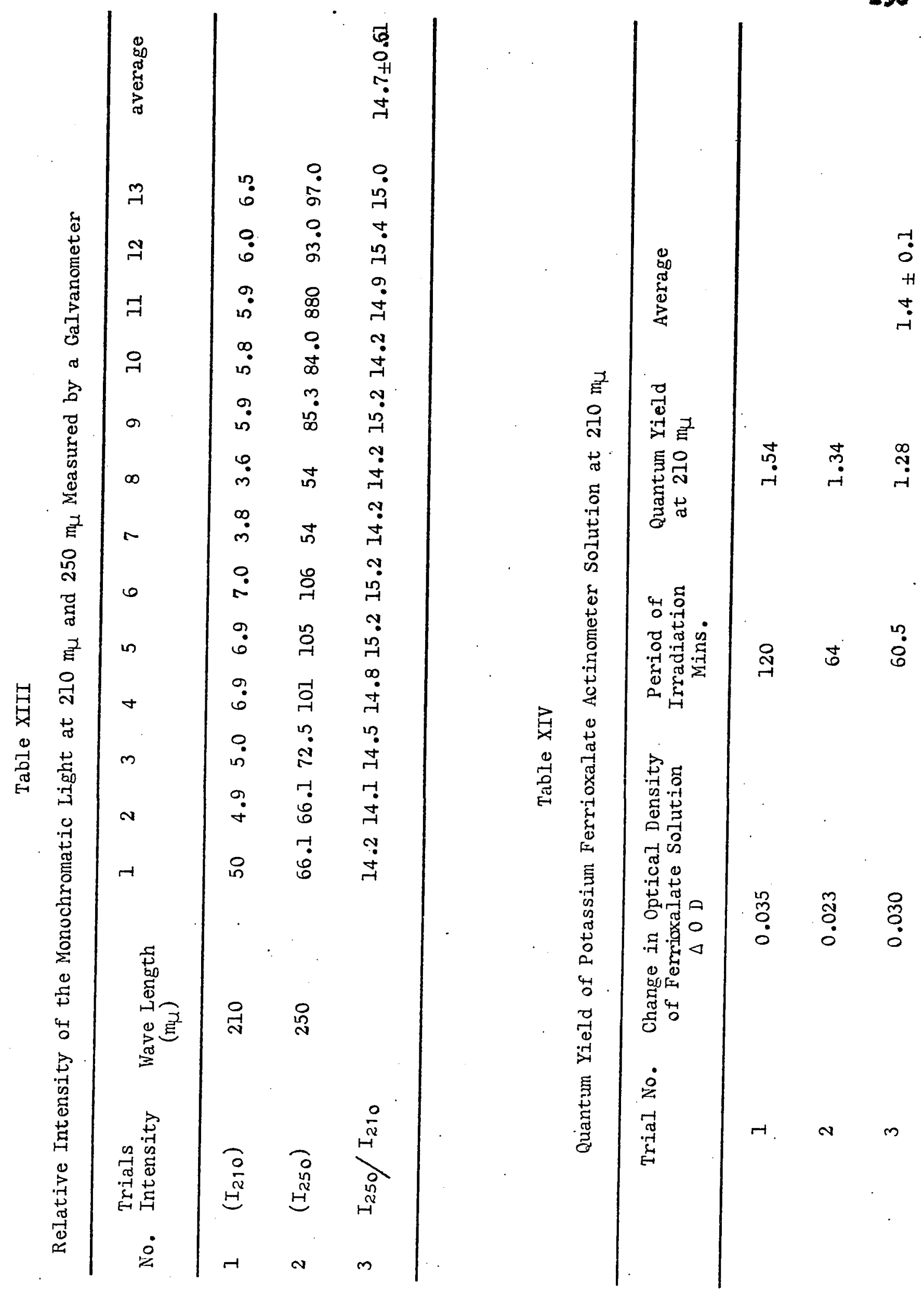
and to that of p-Toluamide, along the absicca, a linear plot was obtained with the equation $Y=0.835 \mathrm{X}+0.0193$ by the Least Square Method. The operating condition of a $101 \times 1 / 81$ column, packed with 80 mesh Chromosorb P coated with $3 \%$ Craig and 1\% Carbowax 20M, were: Column temperature, 170 $171^{\circ}$; pressure, 40 psi; retention time of benzamide, $31.8 \mathrm{~min}$, retention time of $p$-toluamide, 46.5 min.

\section{K. Substituent Effect:}

I. Quantum Yield Measurement of f-Substituted Benzamides

Six different benzaldoximes were selected for investigation, viz. benzaldoxime, p-tolualdoxime, p-anisaldoxime, p-chlorobenzaldoxime, p-bromobenzaldoxime, and p-fluorobenzaldoxime. The concentration of the p-substituted benzaldoxime in cyclohexane was 0.007 to $0.008 \mathrm{M}$ in the presence of glacial acetic acid $(0.73 \mathrm{M})$. The solutions were degassed by the "freezepump-thaw" method at a residual pressure of $0.005 \mathrm{~mm}$ of $\mathrm{Hg}$, sealed under vacuum, and irradiated for approximately $20 \mathrm{hrs}$. at $290 \mathrm{m \mu}$. The intensity of light was monitored in the usual way •

The yields of the p-substituted benzamide were assayed by infra-red spectrophotometry after concentrating the photolyte by removal of solvent at $2 \mathrm{~mm}$. The loss of benzamides employing this technique was determined by ultraviolet spectrophotometry. 
The yield of p-anisamide could not be determined by the method described above; the photolyte contained a mixture of p-anisamide, p-methoxyformanilide, and polymeric material. The residue from the irradiated solution was usually dark brown and rendered the analysis unreliable. A method combining TIC and U.V. spectrophotometry was employed for estimation of p-anisamide and $\mathbb{N}$-p-anisylformanilide. When applied to a plate coated with $0.6 \mathrm{~mm}$ layer of silica gel and eluted a solvent mixture of ethyl ether and petroleumether $(35-60,5: 1)$, the respective $R_{f}$ values of $p$-anisamide N-p-methoxyformanilide were 0.06 and 0.17 . For the analytical purposes, almost complete recovery of p-anisamide could be effected with a 4:I cyclohexane n-propanol solvent system. Since the same technique of quantitative determination was applied to both amides, and considering the fact that the $R_{f}$ value of an amide is a reflection of the strength of the adsorbitivity to the silica gel, it was assumed that $\mathbb{N}$-p-methoxyformanilide could be recovered to the same extent as p-anisamide. The $R_{f}$ value of the latter amide was smaller than that of $\mathrm{N}$-p-methoxyformanilide.

All the solutions which were prepared for infra-red spectrophotometry were recovered and analyzed to PLC for the presence of benzamides and formanilides.

All ultraviolet spectra of various para-substituted benzaldoximes were taken in cyclohexane with a Cary $14 \mathrm{U} . \mathrm{V}$. 
Spectrophotometer. The spectra of the amides were taken in methanol or cyclohexane on either a Cary 14 or a Hitashi-P.E. -139 .

II. Infra-red Spectrophotometry Calibration of p-Substituted Benzamides

A standard solution of benzamide (2-4mg $\left.\mathrm{ml}^{-1}\right)$ was prepared in chloroform (Fisher Spectroscopic) and diluted to different concentrations. A Beckman Infra-red Spectrophotometer-Model IR-7 was adopted for "normal" running under the specific settings: Period-2, Course Gain, 10X; Fine Gain, 0.100; Double Beam; Select operation, Slit $3: 1$ adjusted at $1675 \mathrm{~cm}^{-1}$. Calibration curves were drawn for the benzamides.

III. Iarge Scale Irradiation of p-Fluorobenzaldoxime p-Fluorobenzaldoxime (204mg) and acetic acid (8.4ml) were dissolved in cyclohexane (200ml) and irradiated through Vycor and Corex filters in an immersion well with a 450 watt high pressure mercury lamp. The cut-off was at 257mp. Upon termination of the irradiation, the reaction mixture was concentrated 'in vacuo' and the residue (326mg) was separated by IIC on a plate ( 8 " $x$ 8") coated with silica geI (60gm/ plate) by eluting with a 5:1 mixture of ethyl ether and petroleum-e ther $35-60^{\circ}$. p-Fluorobenzamide band was extracted with ethyl ether, dried over anhydrous sodium sulfate, concentrated and crystallized from ethanol. The yield was 50mg (mp.155-156, Iit. 154.5-155.5). The photochemically produced product was identical in all respects with an 
authentic sample.

Irradiation of the solution of p-fluorobenzaldoxime through quartz with a 80 watt medium pressure lamp did not afford any p-fluorobenzamide when irradiated for a period of time half as long as had been used with the 450 watt lamp through Corex-Vycor filter.

IV. Large Scale Irradiation of $p$-Anisaldoxime

A cyclohexane solution (300ml) of o-p-anisaldoxime $(313 \mathrm{mg})$ and acetic acid (27ml) was prepared. $200 \mathrm{ml}$ of the solution contained in an immersion well was irradiated through Vycor and Corex with a 450 watt high pressure mercury lamp for three hours. The reaction was followed by taking an aliquot periodically during the irradiation and analyzing it spectroscopically. After the irradiation, the solvent and acetic acid were removed at $2 \mathrm{~mm}$ of $\mathrm{Hg}$ and the residue was separated by TLC, using silica gel and eluting with a 5:1 solvent mixture of ethyl ether and petroleum-ether (35-60'). p-Anisamide and p-methoxyformanilide, were extracted with ethyl ether, filtered, concentrated 'in vacuo' and purified. p-Anisamide was crystallized out from ethanol and water and the yield was 53mg (mp.1647650, Iit. $163^{\circ} \mathrm{C}$ ).

$80 \mathrm{ml}$ of the same $\alpha-p$-anisaldoxime solution, when irradiated on an optical bench at $290 \mathrm{~m} \mu$ for 16 hours and 
worked up similarly as above, afforded 44mg of p-anisamide. The yield of this amide was slightly less than that of pmethoxyformanilide.

The purification of p-methoxyformanilide was accomplished by subliming off the residue from the first amide ethereal extract at $130^{\circ} / 2 \mathrm{~mm}$ (bath temperature), rechromatography, and re-subliming the second extract from the TIC tile at 100-110\%.1mm (bath temperature). The physical properties, infra-red and ultraviolet spectra, and the $R_{f}$ values on a TLC tile for both amides which were prepared photochemically were identical with those of authentic samples.

For large scale photochemical preparation of p-anisamide and p-methoxyformanilide, optimum conditions consisted of irradiating a highly concentrated solution of a-p-anisaldoxime for a short period of time to avoid decomposition of the amides. It was found that $25 \mathrm{hrs}$. of irradiation through Corex-Vycor using the 450 watt mercury lamp, did not yield any one of the amides.

V. The Rearrangement of $p$-Methox acetophenone Oxime 1. Iarge Scale Irradiation: p-Methoxyacetophenone oxime ( $3.95 \mathrm{~g}$ or $0.12 \mathrm{M} \cdot)$ and glacial acetic acid ( $24 \mathrm{ml}$ or $22 \mathrm{M}$ ) were dissolved in cyclohexane $(200 \mathrm{ml})$ and irradiated through Vycor-Corex for $71 \mathrm{hrs}$. The solvent was removed under vacuum at $22 \mathrm{~mm}$ of $\mathrm{Hg}$ and the 
residue was chromatographed on six thick layer plates (8". $\mathrm{x}$ 81") which had been coated with a layer of silica gel (20 gm/ plate). Elution with ethyl ether and petroleum ether (35-600) (1:5), conveniently separated the starting material from the photochemically produced amides. The amides were extracted from the silica gel with ethyl ether, concentrated, and subsequently sublimed ( $120-140^{\circ}, / 0.2 \mathrm{~mm}$ bath temperature), affording a mixture (24Img) of $\mathbb{N}$-p-anisylacetamide, N-methylanisamide, and p-anisamide. The three amides were then separated as before on plates coated with silica gel $(0.8 \mathrm{~mm}$ thick), eluted with the same solvent system, and extracteI with ethyl ether. Evaporation of the solvent afforded $N-p-$ anisylacetamide $(103 \mathrm{mg}), \mathbb{N}$-methylanisamide $(50 \mathrm{mg})$ and $\mathrm{p}-$ anisamide ( $10 \mathrm{mg}$ ). N-p-anisylacetamide was recrystallized from methanol and ethyl ether (mpl28-129, Iit. 128-129 , 127-130 ). N-methylanisamide was recrystallized from cyclohexane and ethyl ether (m.p.120-12l, Iit. 120-121 ${ }^{\circ}$, and panisamide from ethanol and cyclohexane (m.p. 163.5-164.5, Iit. 164-165). The $\mathrm{K}_{f}$ values for these three amides on development by ethyl ether and petroleum ether $\left(35-60^{\circ}, 5: 1\right.$ ) were respectively, $0.27,0.19$, and 0.12 . Mixed melting points of photochemically produced amides showed no depression on mixing with authentic samples and the $R_{f}$ values of the three amides from the reaction were also identical with those of the authentic samples. In addition, spectral data confirmed the identity of photochemically and chemically produced amioes. 
The spectral data for $\mathbb{N}$-p-anisylacetamide, N-methylanisamide, and p-anisamide are respectively: IR; $\bar{y} c=0$ at 1690 , and $1673 \mathrm{~m}^{-1}$, and $1673 \mathrm{~cm}^{-1}$ in $\mathrm{CS}_{2}: U . V$. maxima were at $248 \mathrm{~m} \mu$ $(\epsilon=15,800), 250 \mathrm{~m} \mu(\epsilon=15,700)$, and $250 \mathrm{~m} \mu(\epsilon=14,800)$ in cyclohexane-n-propanol ( $4: 1)$.

Unexposed samples having the same concentration as the irradiated samples of $p$-methoxyacetophenone did not afford any detectable amount of amides after standing at room temperature for 71 hours.

2. Quantum Yield Measurements of IN-p-Anisylacetamide, N-methylanisamide, and p-Anisamide:

A stock solution of p-methoxyacetophenone oxime $(0.0043 \mathrm{M})$ in cyclohexane was prepared and a $5.00 \mathrm{ml}$ aliquot was irradiated at $290 \mathrm{~m} \mu$ for $20 \mathrm{hrs}$. All samples were degassed at $.003-.005 \mathrm{~mm}$ of $\mathrm{Hg}$. by the "freeze-pump-thaw" method and sealed under vacuum. Irradiations of four samples were carried out consecutively, two of them containing acetic acid (0.74M) and two containing no acetic acid. The intensity of light was monitored in the usual manner using $10.00 \mathrm{ml}$ of the actinometer solution.

Infra-red spectroscopic technique could not be employed for assaying the yield of amides. Instead, the TLC-U.V. Spectrophotometric method was employed here. After an irradiation, the photolyte was transferred to a $10.0 \mathrm{ml}$ round bottomed flask and was "freeze-dried" for 2-3 hris. at $5 \mathrm{~mm}$ of $\mathrm{Hg}$ 
The residue was taken up in chloroform, spotted on a TIC plate coated with silica gel $(0.75 \mathrm{~mm}$ thick) using the proportion ( $90 \mathrm{gm} / 190 \mathrm{ml}$ of water), and luted with a solvent mixture of ethyl ether and petroleum ether (35-60) (5:1). Each amide band was scraped off into a $25.0 \mathrm{mI}$ volumetric flask, extracted with a solvent mixture (25ml) of cyclohexane and n-propanol $(4: 1)$, allowed to stand for $2 \mathrm{hrs}$, at room temperature, and then centrifuged. The supernatant liquid was subjected to U.V. analysis using a Cary 14 Spectrophotometer. From the pre-determined molar extinction coefficients of the three amides in the same solvent mixture, the yield and the quantum yield were calculated.

The solvent system containing a mixture of cyclohexane and n-propanol $(4: 1)$ was shown to be capable of extracting over $98 \%$ of the amides from silica gel after application of 0.4 to $-0.5 \mathrm{mg}$. Authentic samples of $\mathbb{N}$-p-anisylacetamide, Nmethylanisamide, and p-anisamide were dissolved in ethanol (95\%), spotted on the TIC plate as a band ( $4 \mathrm{~cm}$ Iong), extracted with the solvent mixture, centrifuged and analyzed spectrophotometrically. A comparison with unspotted samples of the amides was made to determine the recovery of amides on the silica gel plate by the methods empluyed for analysis.

I. Solvent Effects: Quantum Yield Measurements of Benzamide

The quantum yield of benzamide from benzaldoxime was 
measured in different solvents in the usual manner. 5.00m I of benzaldoxime solution (0.008M) were degassed at $0.005 \mathrm{~mm}$ of $\mathrm{Hg}$. by the "freeze-pump-thaw" method and irradiated at $290 \mathrm{m \mu}$ for approximately $24 \mathrm{hrs}$. The yield was assayed by infrared spectrophotometry and the intensity of light was monitored in the usual way using a ferrioxolate actinometer solution.

Irradiation of aprotic solvent was conducted in the presence $(0.730$.$) and in the absence of acetic acid.$ 


\section{BIBLIOGRAPHY}

1. P. de Mayo and S.T. Re1d, Quart. Rev. (London) 15, 393 (I961):.

2. O.I. Chapman in Advances in Photochemistry, edit. W.A. Noyes, Jr., G.S. Hammond, I.N. P1tts, Jr., Vol. I, Interscience Publisher, John Wiley and Sons, New York, N.Y., 1963, p.323.

3. A. M. Small, Chem. Comm. 243 (1965).

4. S. Masumune and N.T. Castelluc1, Proc. Chem. Soc. (London) 298 (1964).

5. W.J. Dauben and W.T. W1pke, J. Pure and Appl. Chem. 2, 539 (1964).

6. J. Wlemann, N. Thoal and F. Welsbuch, Tetrahedron Letters, 2983 (1965).

7. M.J. Jorgenson, Chem. Comm. 137 (1965).

8. P. de Mayo, J. Pure and Appl. Chem. 2, 597 (1964).

9. N. Obata and. J. Moritant1, Tetrahedron Letters 1503 (1966).

10(a) H. Prinzbach and A. Hagemann, Angew. Chem. (Internat. edit.) 3,653 (1964).

(b) H. Prinzbach, H. Hagemann, J.H. Hartenstein, and R. K1tz1g, Ber. 28, 2201 (1965).

(c) H. Wynberg and H. Van Driel, Chem. Comm. 203 (1966).

(d) J. Meinwald, A. Eckell, and K.I. Erickson, J. Am. Chem. Soc. 87, 3532 (1965).

II(a) H. E. Zimmerman in Advances in Photochemistry, edit. W.A. Noyes, Jr., G.S. Hammond, J.N. P1tts, Jr., Vol. I, Interscience Publisher, John Wiley and Sons, New York, N.Y., 1963, p.183.

(b) H.E. Zimmerman, J. Pure and Appl. Chem. 2, 493 (1964).

(c) D.I. Schuster and D.J. Patel, J. Am. Chem. Soc. 88, 1825 (1966).

(d) H.E. Zimmerman, R.C. Hahin, H. Morrison, and M.C. Wan1, J. Âr. Chem. Soc. 87, 1138 (1965). 
12(a) D.H. Barton, P. de Mayo, and M. Shafiq, J. Chem. Soc. 929 (1957).

(b)P.J. Kropp, J. Am. Chem. Soc. 87, 3914 (1965).

(c)P.J. Kropp, Tetr. 21, 2183 (1965).

13(a)H. E. Z1mmerman, R.G. Lewis, J.J. MeCullough, A. Padwa, S.W. Staley and M. Semmelhack, J. Am. Chem. Soc. 88, 1965 (1966).

(b)E.F. Ullman and W.A. Henderson, Jr., J. Am. Chem. Soc. 86, 5051 (1964).

(c)B.A. Shoulders, W.W. Kw1e, W. Klyne, and P.D. Gore, Tetr. 21, $2973(1965)$.

14(a) J.G. Calvert and J.N. P1tts, Jx., Photochem1stry, John W1ley and Sons Inc., NeW York, N.Y. 1966, p.379.

(b) R.G. Norrish, H.G. Crone and O.D. Saltmarsh, J. Chem. Soc. 1456 (1934).

(c)W.A. Nozes, Jr., and I. Unger, J. Pure and Appl. Chem. 2, 461 (1964).

15(a) H. Kobsa, J. Org. Chem. 27, 2293 (1962).

(b) J.C. Anderson and C.B. Reese, Proc. Chem. Soc. 217 (1960).

(c) J.C. Anderson and C.B. Reese, J. Chem. Soc. 1781 (1963).

(d)R.A. Finnegan and J.J. Mattice, Tetr. 21, 1015 (1965).

(e)D. Taub, C.H. Kuo, H.I. Slates, and N.I. Wendler, Tetr. 19, 1 (1963).

16(a) D. Elad, D.V. Rao, and V.I. Stenberg, J. Org. Chem. 20, 3252 (1965).

(b) R.O. Kan, and R.L. Furey, Tetrahedron Letters 2573 (1966).

17. H. E. Hoffmelster and D.S. Tarbell, Tetr. 21, 35 (1965).

18. H. Nozak1, T. Okada, R. Noyor1, and M. Kawan1si, Tetr. 22, 2177 (1966).

19(a) M. Kharasch, C. Stampa and. W. Nudenberg, Science 116, 309 (1952).

(b)J.T. PInhey and K. Schaffner, Chem. Comm. 519 (1966). 
20(a)R.A. Finnegan and A.W. Hagen, Tetrahedron Letters 365 (1963).

(b) M. Feldk1mel-Gorodetsky and Y. Mazur, Tetrahedron Letters 369 (1963).

(c)A. Yoger, Feldk1mel-Gorodetsky, and. Y. Mazur, J. Am. Chem. Soc. 86, 5208 (1964).

(d) M. Feldk1mel-Gorodetsky, and Y. Mazur, J. Am. Chem. Soc. 86,5213 (1964).

21. G. M. Coppinger and E. R. Bell, J. Phys, Chem. 20, 3479 (1966).

22. H. E. Zimmerman, B.R. Corley, C.Y. Tseng, and J.W. W11son J. Am. Chem. Soc. 86, 947 (1964).

23. C.K. Johnson, B. Doming and W. Beusch, J. Am. Chem. Soc. 85,3894 (1963).

24. C. Lehmann, K. Schaffrer, and O. Jeger, Helv. Ch1m. Acta. 45, 1031 (1962).

25. S. Bodforss, Chem. Ber. 51, 214 (1908).

26. A.N. Strachan, and F.E. Blacet, J. Am. Chem. Soc. 27, 5254 (1954).

$27(a) C$. Weizmann, Y. Hirsberg, and. E. Bergman, J. Am. Chem. Soc. 58, 1675 (1936).

(b) C. Welzmann, Y. Hirsberg, and E. Bergmann, J. Am. Chem. Soc. 60, 1799 (1938).

(c) I. Mandel and A. D. McLaren, J. Am. Chem. Soc. 23, 1826 (1951).

28(a)L. Farkas, Z. Physik, Chem. (Le1pz1g) 23, 89 (1933).

(b)J.C. Anderson and C.B. Reese, Tetrahedron Letters 1 (1962).

(c) J.J. Hlavka and H.M. Krazinsk1, J. Org. Chem. 28, 1422. $(1962)$.

$29(a)$ H. Wehrli, M. Cereoghett1, K. Schaffner, and O. Jeger, Helv. Chim. Acta. 43, 367 (1960).

(b)A. Ferguson, P. de Mayo, F.L.M. Pattison and T. Tabata, Can. J. Chem. 41, 2099 (1962).

30. R. Warszawsk1, K. Schaffner, and O. Jeger, Helv. Ch1m. Acta. 43, $500(1960)$. 
31. C. Ganter, B. Warszawsk1, H. Wehrl1, K. Schaffner, o. Jeger, Helv. Chim. Acta. 40, 320 (1963).

32 (a) H. E. Z1mmerman, and D.I. Schuster, J. Am. Chem. Soc.

(b) H. E. Zimmerman, Tetrahedron Suppl. 2 19, 393 (1963).

33(a) M.S. Kharasch, A. Fono and W. Nudenberg, J. Org. Chem.
16, 113 (1951). (b) C. Walling, Free Radicals in Solutions, John Wiley and
Sons, Inc., NeW York, N.Y. 1957, p.505.

34. C. Walling in Molecular Rearrangement edit. P. de Mayo, Part I, Interscience Publisher Inc., New York, N.Y.
1963 , p. 416 .

35. R.Kh. Fellinda in Adjances in Free Radical Chemistry, ed. G. H. Williams, Vol. I Logos Press Lta., London, 1965,
p.2il.

36. A. Wehrl1, C. Lehman, K. Schaffner and O. Jeger, Helv.
Ch1m. Acta. $47,1336(1964)$.

37. O. Jeger, K. Schaffner and H. Wehrl1, J. Pure and Appl.
Chem. 2, 555 (1964).

38(a)L.H. Slaugh, J. Am. Chem. Soc. 81, 2262 (1959).

(b) D.Y. Curtin and J.C. Kauer, J. Org. Chem. 25, 880 (1960). 39(a) G.W. Griffin, J. Covell, R.C. Petterson, R.M. Dodson, and
G. Close, J. Am. Chem. Soc. 87,1410 (1965).

(b) H. Kristinsson and G.W. Griffin, J. Am. Chem. Soc. 88,
378 (1966).

(c) G.W. Griffin, A.F. Marcanton10, H. Kristinsson, and R.C. Peterson, Tetrahedron Letters 2951 (1965).

(d) H. Kristinsson, R.A. Mateer and G.W. Griffin, Chem. Comm.
415 (1966).

(e)H. Kristinsson and G.W. Griffin, Tetrahedron Letters 3259
(ig66).

(f) H. Kristinsson and G.W. Grifrin, J. Am. Chem. Soc. 88 ,
378 (1966).

40 (a) D.I. Schuster and C.J. Polowczyk, J. Am. Chem. Soc. 88,
1722 (1966). 
40(b) D.I. Schuster and C.J. Polowczyk, J. Am. Chem. Soc. 86, 4502 (1964).

41. D.I. Schuster and I.S. Krull, J. Am. Chem. Soc. 88, 3456 (1966).

42. L.P. Tenney, D.W. Boykin, Jr., and R.E. Lutz, J. Am. Chem. Soc. 88, 1835 (1966).

43. L.B. Rodwald and C.H. DePuy, Tetrahedron Letters, 2951 (1964). 44. J.N. P1tts, Jr., and I. Norman, J. Am. Chem. Soc. 26,

45. G.S. Hemond, P. Wyatt, C.D. DeBoev, and N.J. Turro, J. Am. Chem. Soc. 86, 2532 (1964).

46. J.A. Bell, J. Am. Chem. Soc. 87, 4966 (1965).

47. II.J. Cretanov1c and I.C. Doyle, J. Chem. Phys. 3i, 543 (1962).

48(a)R. Srinivasan, J. Phys. Chem. 67, 1367 (1963).

(b) B. Srinivasan, J. Am. Chem. Soc. 86, 3318 (1964).

49. G.W. Griffin, E.J. O'Connell, and H.A. Hammond, J. Am. Chem. Soc. 85, 1001 (1963).

50. R.C. Cookson, N.J. Nye and G. Subrahanyan, Proc. Chem. Soc. 144 (1964).

51(a)H. Kristinsson and G.W. Griffin, Angew. Chem. (Internat. edit.) 4,868 (1965).

(b) H. Kristinsson and G.W. Griffin, J. Am. Chem. Soc. 88, 1579 (1966).

52. L. Wacker, Ann. 217, 375 (1901).

53. O. Baudisch and R. Furst, Ber. 45, 3426 (1912).

54. H.M. Knipscheer, Rec. trav. chim. 22, 1 (1903).

55. W.M. Cunningham and J.K. Steel, J. Chem. Soc. 123, 2464 (1923).

56. W.M. Cunningham and G.S. Ferrier, J. Chem. Soc. 127, 2374 (1925).

57. W. M. Cunningham and G. Howie, J. Chem. Soc. 3181 (1931). 58(a)G.M. Badger and R.G. Buttery, J. Chem. Soc. 2243 (1954). 
58(b) G. E. Lew1s and J.A. Re1ss, Aust. J. Chem., 19, 1887 (1966).

59. A. Schonberg, in Preparative Organische Photochemie,
Springer, Berlin, 1958, $19-21$

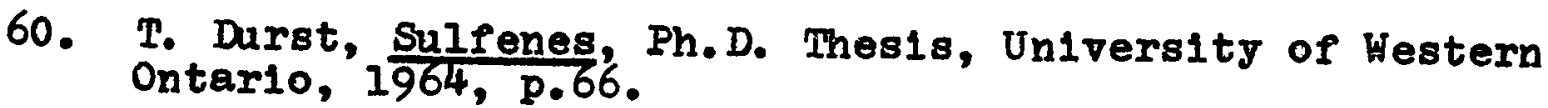

61. J.F. King and T. Durst, J. Am. Chem. Soc. 85, 2676 (1963).

62. J. Strating, I. Thijs, and B. Zwanenburg, Rec. trav. ch1m., 83, 631 (1964).

63. P. de Mayo and J. Byan, Tetrahedron Letters, 827 (1967). 64. I. Atessandri

(a)Att1 accad.nazl.Lince1, Mem.Classe-sc1.fis.mat nat.Sez, $19, \underline{2}, 122$ (1910).

(b) Chem. Zentr. 2, 1043 (1910).

(c)Chem. Abstr. 5, 276 (1911).

65. O. Brady and A. McHugh, J. Chem. Soc. 125, 547 (1924). $66(a)$ I. Chardonnens and P. Heinrich, Helv. Chim. Acta. 32 ,
656 (1949).

(b) J. Landquist, J. Chem. Soc. 2830 (1953). (c) M.J. Haddadin and C.H. Issidorides, Tetrahedron Letters,
753 (1967).

67(a)J. Splitter and M. Calvin, J. Org. Chem. 20, 1086 (1955).

(b)J. Splitter and. M. Calvin, J. Org. Chem. 23, 651 (1958).

(c)J. Splitter and M. Calvin, J. Org. Chem. 30, 3427 (1965).

68. K. Shinzawa and I. Tanaka, J. Phy. Chem. 68, 1205 (1964).

69. K. Koyano and I. Tanaka, J. Phy. Chem. 69, 2545 (1965).

70. M. Kamlet and I.A. Kaplan, J. Org. Chem. 22, 576 (1957).

71. F. Krohnke, Ann. 604, 203 (1957).

72. A. Padwa, Tetrahedron Letters, 2001 (1964).

73. B.M. Markha1lov and G.S. Ter-Sarkinsyan, Bull. Acad. Sc1. (USSR), 656 (1954). 
74(a) R. Bonnett, V.H. Clark, and A. Todd, J. Chem. Soc. 2102 (1959).

(b)L.S. Kamingky and M. Lamchen, J. Chem. Soc. C, 2295 (1966).

75. I. Brehm, F. G. Jensen, and B. Jerslev, Acta Chem. Scand. 20, 915 (1966).

76. H. Brehm, K.P. Hansen, and B. Jerslev, Acta Chem. Scand. 19, $2166^{\prime}(1965)$.

77. W. D. Ammons, J. Am. Chem. Soc. 28, 6208 (1956).

78. W. D.

79. I. Horner and E. Jurgens, Ber. 20, 2184 (1957).

80. H. Krimm, Ber. 21, 2057 (1958).

81. I.H. Sternbach, B.A. Koechlin, and E. Reeder, J. Org. Chem. 27, 4671 (1962).

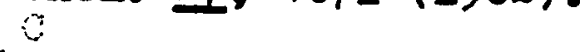

82(a)J.K. Landquist and G.J. Stacey, J. Chem. Soc. 2828 (1953).

(b) C. Kaneko, I. Yokoe, S. Yamada, and M. Ishikawa, Chem. Pharm. Buli. (Japan) 14, 1316 (1966).

83. G. S. Brown, G. Levin and S. Murphy, Blochem. 2, 880
(1964).

84. F. Cramer and G. Schlingloff, Tetrahedron letters, 3201

85(a)M. Ishikawa, S. Yamada, and C. Kaneko, Chem. Pharm. Bull. (Japan) 13, 747 (1965).

(b) M. Ishikawa, S. Yamada, H. Hotta, and C. Kaneko, Chem. Pharm. Bull (Japan) 14, 1102 (1966).

(c)C. Kaneoko, S. Yamada, and M. Ishikawa, Tetrahedron Letters, 2145 (1966).

(d) C. Kaneko and s. Yamada, Chem. Pharm. Bull. (Japan) 14,
555 (1966).

(e) C. Kaneko, S. Yamada, and I. Yokoe, Tetrahedron Letters,
4701 (1966). 86(a)0. Buchardt, Acta Chem. Scand. 17, 1461 (1963).

(b)o. Buchardt, J. Becher, and C. - shse, Acta Chem. Scand. 19, 1120 (1965). 
86(c)0. Buchard.t, J. Becher, and C. Lohse, Acta Chem. Scand. 20, 262 (1966).

(d) 0. Buchardt, and C. Lohse, Tetrahedron Letters, 4355 (1966).

(e)0. Buchardt, Tetrahedron Letters, 6221 (1966).

(f)0. Buchardt, J. Becher, and C. Lohse, Acta Chem. Scand. 20, 2467 (1966).

$87(a) \mathrm{J}$. Streith and C. S1gwalt, Tetrahedron Letters, 1347 (1966).

(b)J.S. Strelth, H.K. Darrah, and M. Well, Tetrahedron Letters, 5555 (1966).

(c)N. Ikekawa and Y. Honma, Tetrahedron Letters, 1197 (1967). 88(a)N. Hata, Bull. Chem. Soc. (Japan) 34, 1440 (1961).

(b)N. Hata, Bull. Chem. Soc. (Japan) 34, 1444 (1961).

(c)N. Hata and T. Tanaka, J. Chem. Phys. 36, 2072 (1962).

89. J.H. Amin and P. de Mayo, Tetrahedron Letters, 1585 (1963).

90. R. T. Taylor, M. Douek, and G. Just, Tetrahedron Letters, $4143(1966)$.

91(a)H. Beckmann, Ber. 19, 988, (1886)。

(b) H. Beckmann, Ber. 20, 1507, (1887).

92. G. Donaruma and W.H. Heldt in Organic Reactions, edit. A.C. Cope, Vol. XI, John Wiley and Sons Inc., New York, N.Y. (1960) P.I.

93. P.A.S. Smith in Molecular Rearrangement, edit. P. de Mayo, Part Je intersc̈ence, John Wlley and Sons, New York, N.Y. 1963 , p.483.

94. I.T. Glover and V.F. Raeen, J. Org. Chem. 31, 1987 (1966).

95. A. Campbell and. J. Kenyon, J. Chem. Soc. 25 (1946).

96. J. Kenyon and D.P. Young, J. Chem. Soc. 263 (1941).

97. G.P. Miklukhin and A.I. Brodsk11, Acta Physico Chim. (USSR) 16, 63 (1942).

98. A.W. Chapman and C.C. Howis, J. Chem. Soc. 2, 806 (1933). 
99. I.A. Neiman, V.I. Maimind, and M.M. Shemyakin, Tetrahedron Letters, 3157 (1965).

100. Y. Hirshberg and E. Fisher, Rev. So1. Instr. 20, 197 (1959).

101. L.J. Lohr and B.W. Warren, J. Chromatog. 8, 127 (1962). 102. J. Cason and E. R. Harr18, J. Org. Chem. 24, 676 (1959).

103. J.M. Bobbitt, Thin Layer Chromatography, Belnhold Publishing Co., New Yoxk, N.Y., 1963, P.117.

104. B. D. Spencer and B.H. Beggs, J. Chromatog. 21, 52 (1966).

105. H.I. B1rd, Jr., H.F. Brlckly, J.P. Comer, P. E. Hartsaw, and M.L. Johnson, Anal. Chem. 25, 346 (1963).

106. M. Hranisavijeric-Jakovljeric, I. Pejkovic-Tadic and A. Stoj11jkov1c, J. Chromatog. 12, 70 (1963).

107. A. E. Gillman and E.S. Stern, An Introduction to Flectronlc Absorption Spectroscopy, E. Amold (Publisher) Ltd.,

108. J.E. Leffler and E. Grunwald, Rates and Equilibria of Organ1c Reactions, John Wiley and Sons, Inc., Ner York,

109. C. Relchardi, Angew. Chem. (Internat. edit.) 4, 28 (1965).

110 (a) E. Schmitz, Advances in Heterocyclic Chemistry, edit. A. R. Katritzky Vo1. 2, Academic Press (London), 1963,
p.83.

(b)E. Sohmitz, Angew. Chem. (Internat. edit.) 3, 333 (1964).

(c) E. Schmitz, B. Ohme, S. Schramm, Tetrahedron Letters, 1857
$(1965)$.

(d)E. Schmitz, Private communication.

111. E. Schmitz, Ber. 28, 2516 (1965). 112. H. F. Edujee, D.M. Newitt, and K.E. Weule, J. Chem. Soc.
3086 (1951).

113. H. Shindo and B. Umezawa, Chem. Pharm. Bull. (Japan) 10,
492 (196́).

114. A. Padra, J. Amer. Chem. 87, 4365 (1965). 115. G. Porter and F. W11k1nson, Proc. Roy. Soc. (London)
A264, 1 (1961). 
116. A.W. Terenin and V.I. Ermolaev, Trans. Faraday Soc. 52,
$1042(1956)$. 117 (a) H.L.J. Backstrom and K. Sandros, Acta Chem. Scand. 12,
823 (1958).

(b) H.I.J. Backstrom and K. Sandros, Acta Chem. Scand. 14,

(c)H.I.J. Backstrom and K. Sandros, Acta Chem. Scand. 16, $958(1962)$.

118. G.S. Hammond, J. Saltiel, A.A. Lamola, N.J. Turro, J.S. Bradshww, D.O. Cowan, R. C. Counsell, V. Vogt, and C. Dalton, J. Am. Chem. Soc. 86, 3197 (1964).

119. G. Clamician and P. Silber, Ber. 36, 4268 (1903).

120. B. Stoormer, Ber. 44, 667 (1911).

121. O.I. Bradiy and F.P. Dunn, J. Chem. Soc. 103, 1620 (1913).

122. O.I. Bradiy and G.P. McHugh, J. Chem. Soc. 125, 547 (1924).

123. J.T. Dubois and F. W1lkinson, J. Chem. Phys. 28, 2541

124. J.T. Dubo1s and B. Steven, in Luminescence of Organ1c and Inorganic Matorials, edit. H.P.K. Kallmann and G.M. Spruch, John Wiley and Sons, New York, N.Y. 1962, p.i15.

125. D.F. Evans, J. Chem. Soc. 1351 (1957).

126. A.A. Lamola and G.S. Hammond, J. Chem. Phys. 43, 2129
$(1965)$.

127. K. Bredereck, Th. Förster and H. G. Oesterlin, in Luminescience of Organic and Inorganic Materials, edit. H.P.K. Kallemann and G.M. Spruch, John Wiley, and. Sons,
New York, N.Y., 1962, p.I61.

128. Y. Kanada, H. Kaseda, and T. Matsumura, Spectrochlm1ca acta 20, 1387 (1964).

129. R. Huisgen, W. Rapp, I. Ug1, H. Walz, and E. Mergenenthaler,
Ann. 286, 1 (ig54).

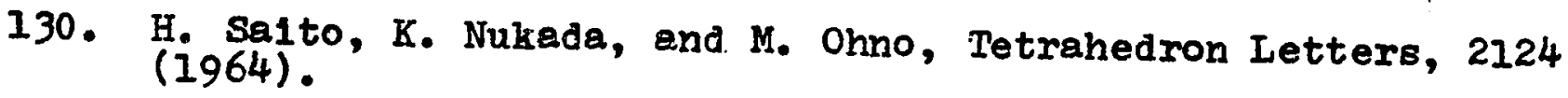

131. G.N. Lewis and M. Kasha, J. Am. Chem. Soc. 66, 2100 (1944). 
132. F. Wilkinson, in Advances in Photochemistry, edit, W.A. Noyes, Jr., G.S. Hammond, J.N. P1tts Jr., Vol. 3, Intersclence Pub., John Wiley and Sons, New York, N.Y., 1964, p.248.

133. L. Jablonsk1, Z. Phys1k. 24, 98 (1935).

134. P. Debye, Trans. Electrochem. Soc, 82, 265 (1942).

135. C.N. Lewis, D. Lipkin, and T. Magel, J. Am. Chem. Soc. 63, 3005 (i941). 136(a) C.A. Parker and C.G. Hatchard, Trans. Faraday Soc. 57 ,
1894 (1961). (b)C.A. Parker and C.G. Hatchard, J. Phys. Chem. 66, 2506
(i962).

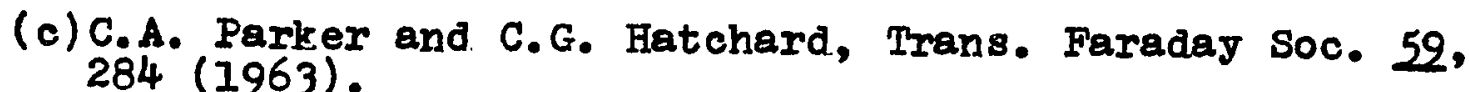
(a) C.A. Parker and C.G. Hatchard, Proc. Chem. Soc. 386
(1962).

(e)C.A. Parker, Proc. Roy. Soc. (Loñon) 1276, 125 (1963).

(f)C.A. Parker and T.A. Joyce, Chem. Comm. 108 (1966).

(G) C.A. Prrker, C.G. Hatchard and T.A. Joyce, J. Mol. Spect. 14, $311(1964)$.

(h)C.A. Parker and T.A. Joyce, Chem. Comm. 234 (1966).

(1)C.A. Parker and C.G. Hatchard, Analyst 87, 672 (1962).

137. V.I. Ermolaev, Usp. Fiz Nauk, 80, 3 (1963), Soriet Phys. Ups. 6, 333 (i963). 138. R.F. Borkman and D. B. Kearns, J. Chem. Phys. 44,945
$(1966)$.

139. R.W. Y1p, Research Lecture, University of Western Ontario,

140. R.F. Borkman and R. Kearns, J. Am. Chem. Soc. 88, 3467
$(i 966)$.

141. W. G. Herkstroet刃r, A.A. Lamola, and G.S. Hammond, J. Am. Chem. Soc. 86, 4537 (1964).

142. W. Noyes, Jr., and I. Unger in Advances in Photochemistry, edit. W.A. Noyes, Jr., G.S. Hammond, J.N. P1tts, Jr., Vol. 4, Interscience Pub., John Wiley and Sons, Now York, 
143. G.S. Hammond, N.J. Turro, and P.A. Leermaker, J. Phys. Chem. 66, 1144 (1962).

144. W.A. Noyes, G.B. Porter and J.E. Jolly, Chem. Rev. 56, 49 (1956).

145. H. B. Klevens and J.R. Platt, J. Chem. Phys. 17, 470 (1949).

146. E. Elar, Aromatische Kohlenwasserstoffe Springer-Verlag, Berlin, 1952.

147. G.J. Bowen, Chemical Aspect of Light, University Press, Oxford, 1940 , p.162.

148. M.R. Phadhye, S.P. McGlynn, and M. Kasha, J. Chem. Phys. 24, 588 (1956).

149. W.W. Bobertson, J.F. Mus1c, and F.A. Matsen, J. Am. Chem. Soc. 72, 5260 (1950).

150. E.J. Pozlomek, J. Pharm. Sc. 45, 333 (1965).

151. R. Srinivasan, J. Am. Chem. Soc. 84, 3432 (1962).

152. G. Z1mmerman, I-Y. Chow, N-J, Paik, J. Am. Chem. Soc. 80, 3528 (1958).

153. W. Swietoslawk1 in Ostwald Druecker Handbuch der allgmeinen Chemie, Leipzig, 1928, p.200.

154. H.H. Jaffe and M. Orchin, Theorg and Applications of Uitraviolet Spectroscopy, John Wiley and Sons, Inc., New York, N.Y. 1962, P.277.

155. S. Malkin and E. Fischer, J. Phys. Chem. 68, 1153 (1964).

156. H. Stegemejer, J. Phys. Chem. 66, 2555 (1962).

157. T.S. Patterson and A. McMillan, J. Chem. Soc. 505 (1924).

158. E. Fischer and Y. Fre1, J. Chem. Phys. 27, 808 (1957).

159 (a) G. Wettermark and I. Dogliott1, J. Chem. Phys. 40, 1486 (1964).

(b) G. Wettermark, J. Winstein, J. Sousa, and L. Dogllott1, J. Phys. Chem. 69, 1584 (1965).

$I 60$ (a) S. Gladstone, K. Laldler, and H. Eyring in The Theory of Bate Processes, McGraw Hill, New York, N.Y. 1941, p.323.

(b) J.L. Magee, W. Strand, and H. Eyring, J. Am. Chem. Soc. 63, 677 (1941). 
161. H.L.J. Backstrom and K. Sandros, J. Chem. Phys. 23,
2193 (1955).

162. R. Livingston and D.W. Tannes, Trans. Faraday Soc. 54 ,
765 (1958).

163. A.I. Vogel, A Test Book of Practlcal Organic Chemistriy, Longmans, Green, and Co., Toronto, 1956,

164. D.R. Kearns, J. Phys. Chem. 69, 1062 (1965).

165. I. B. Jones and G.S. Hammond, J. AM. Chem. Soc. 87, 4219
$(1965)$.

166. W.F. Linke, Solubilities of Inorganic and Metal-Organ1c Compounds, American Chemical Soc1ety, Vol. 2, Hashington,

I67(a) E.J. Bowen and A.H. W1lliams, Trans. Faraday Soc. 35, 1,
765 (1939).

(b) E. J. Bowen, Trans. Faraday Soc. 50, 97 (1954).

(c) E.J. Bowen in Advances in Photochemistry, edit. W.A. Noyes Jr., G.S. Hammond, J.N. P1tts, Jr., Vol. 1 , Interscience Pub., John Wiley and Sons, New York, N.Y. p. 38 .

168. B.I. Funt and. E. Neparko, J. Phys. Chem. 60, 267 (1956).

169. E. W1gner, Nachr. Ges. W1ss. Gottingen Math Physik KI,
375 (1927).

170. J.T. Vanderslice, E.A. Mason, and W. G. Ma1sch, J. Chem。 Phys. 32, 515 (1958).

171. D.F. Evans, J. Chem. Soc. 1735 (1.960).

172 (a)P. Grammaticakis, Bull. Soc. Chim (France) 20, 821
(1952).

(b)P.E. Stevenson, J. Mol. Spectr. 15, 220 (1965).

173(a) Mme. Ramart-Lucas, Bull. Soc. Chtm (France) (5) 1, 719
(1934).

(b) Mme. Ramart-Lucas, Bull. Soc. Ch1m (France) (4) 51,119
(1932).

(c) R.Y. Mizer, R.F. Heck, S. Welnstein, and W.G. Young,

174. H. Suzuk1, Bull. Chem. Soc. (Japan) 33, 613 (1960). 
175. J. Tanaka, S. Nagakura, and M. Kobayash1, J. Chem. Phys. 24, 311 (1956).

$176(a)$ I. Doub and J.M. Vandenbelt, J. Am. Chem. Soc. 69, 2714 $(1947)$.

(b)I. Doub and J.M. Vandenbelt, J. Am. Chem. Soc. 21, 2414 (1949).

177. E. Borello, Advances in Molecular Spectroscopy, edit. A. Mangini, The MacMlilan Co., New York, N.Y. Vol. 1, 1962, p.365.

178(a)T. Kubota, M. Yamakara, and Y. Mor1, Bull. Chem. Soc. (Japan) 36, 1552 (1963).

(b) Y. Kabota and M. Yamakawa, Bull. Chem. Soc. (Japan) 36, 1564 (1963).

179. Y. Yoshihara, and D. R. Kearns, J. Chem. Phys. 45, 1991 (i966).

180. J.F. Music and F.A. Matsen, J. Am. Chem. Soc. 22, 5256 (1950).

181. J. Petruska, J. Chem. Phys. 24, 1120 (1961).

182(a)P.A. Leermaker and H.T. Thomas, J. Am. Chem. Soc. 87, 1620 (1965).

(b)M. Robin and R.N. Trueblood, J. Am. Chem. Soc. 29, 5138 (1957).

183(a) G.H. Booth and R.G.W. Norr1sh, J. Chem. Soc. 188 (1952).

(b) B.C. Spall and E.W.R. Steacle, Proc. Roy. Soc. (Iondon) 239, 1 (1957).

184. C.H. Hatchard and C.A. Parker, in Advances in Photochemistry, edit. W.A. Noyes, Jr., G.S. Hammond, and J.N. P1tts, Jr., Vol. 2, Intersclence Pub., John W1ley and Sons, Inc., New York, N.Y. 1964, p.337.

185(a)K. Clusius and W. Schanzer, Ber. 25B, 1795 (1942).

(b)P. Ausloss and E.W.R. Steacie, Can. J. Chem. 23, 1530 (1955):

186. C.A. Parker and C.G. Hatchard, Trans. Faraday Soc. ㄱ, 1894 (1961).

187. R. Bauer and A. Baczynksk1, Bull. Acad. Polon. Sc1. 6, 113 (1958). 
188. Y.K. Syrkin and M.E. Dyatkina, Stmeture of Molecules and the Chem1cal Bonds, Dover Publication, New York, N.Y.

189. C.A. Swan and P.S. T1mmons, J. Chem. Soc. 1120 (1962).

190. G. Just and C. Pace-Asciak, Tetr. 22, 1069 (1966).

191. I.A. Paquette, Tetrahedron Letters, 485 (1962).

$1.92(a)$. Hormer and A. Chr1stmann, Angew. Chem. (Internal. edit.) 2, 599 (1962).

(b)A. Christmann, PhD. Thesis, Universitat, Mainz (1961).

193. J.N. P1tts, H.W. Johnson, Jr., and T. Kawana, J. Phys. Chem. 66, 2456 (1962).

194. G.S. Hammond and P.A. Leermaker, J. Am. Chem. Soc. 84,
207 (1962).

195. N.C. Yang, Pure and Appl. Chem. 2, 591 (1964).

196. N.C. Yang, M. Nussim, M.J. Jorgenson, and S. Murov, Tetrahedron Letters, 3652 (1964).

197. D. R. Armold, R.I. Hinmen, and A.H. Glick, Tetrahedron Letters, 1425 (1964).

198. G. Porter and P. Suppan, Pure and Appl. Chem. 2, 499

199. G. Porter and P. Suppan, Trans. Faraday Soc. 61, 1664 (1965).

200. A. Weller in Progress in Reaction Kinet1cs, edit. G. Porter, Vol. 1, Pergamon Press, Ner York, N.Y. 1961, p.187.

201. G. Jackson and G. Porter, Proc. Roy. Soc. (Iondon) A260,
13 (1961).

202. H.H. Jaffe and H.L. Jones, J. Org. Chem. 30, 964 (1965).

203. J.C. Haycock, S.F. Mason, and B. Sm1th, J. Chem. Soc.

204(a) E.M. Arnett in Progress in Physical Chem1stry, edit. S. G. Cohen, A. Stritwe18ser, Jr., and R.W. Taft, Interscience, New York, N.Y. Vol'. 1, 1963, p.349

(b)K. Freudenberg, Stereochem1e, Jelpz1g, 1933 p.980.

(c) A. Friedolsheim, Ann。 449, 109 (1926). 
205. E.I. Wehry and L.B. Rogers, J. Am. Chem. Soc. 87, 4234 (1965).

206. H. Ziffer and N.E. Sharpless, J. Oxg. Chem. 27, 1944 (1962).

207. G.C. Phllbrook and M.A. Maxwell, Tetrahedron Letters, 1111 (1964).

208. A. Padra and R. Layton, Tetrahedron Letters, 2167 (1965).

209. U. Mazzucato, G. Gauzzo and A. Forfan1, Tetrahedron Letters, 1525 (1963).

210. W. Kirmse and I. Horner, Ann. 625, 34 (1959).

211. H.G. Bryce, in Fluorine Chemistry, edit. J.H. Simon, Vol. 5, Academic Press, New York, N.Y. 1964, p.490.

212. E.W. Crandall and J. Olguin, J. Org. Chem. 3l, 972 (1966).

213 (a)P.S. Danner and J.H. Hildebrand, J. Am. Chem. 44,2824 (1922).

(b)W.K.J. McEran, J. Am. Chem. Soc. 58, 1124 (1936).

(c)J. Hine and M. Hine, J. Am. Chem. Soc. 24, 5266 (1952).

214. I.M. Kolthoff and S. Bruckenstein, J. Am. Chem. Soc. 28 , 1 (1958).

215. S.J. Bhoads, in Molecular Rearrangement, edit. P. de Mayo, Part I, John Wiley and. Sons, Inc., New York, N.Y. 1963, p.655.

216. E.C. Taylor and G.G. Spence, Chem. Comm. 767 (1966).

$217(a) J_{.} D_{0}$ Roberts and. J.A. Yancey, J. Am. Chem. Soc. 24, 5943 (1952).

(b) J.D. Roberts and C.M. Regan, J. Am. Chem. Soc. 25, 2069 (1953).

(c) J.D. Roberts and M. Halman, J. Am. Chem. Soc. 25, 5759 (1953).

218. W.D. Emmons, in Heterocyclic Compounds with Three- and Four-Membered Rings, edit. A. Welssberger, Part ?. Interscience, Nex York, N.Y. 1964, p.624.

219. K. Nakanish1, Infrared Absorption Spectroscopy, Holden Day, Inc., San Francisco, Cal., 1963. 
220. F.D. Greene, W. Adam, and G.A. Knudson, J. Org. Chem. 31, 2087 (1966).

221(a)A. Van T1ggelen, J. Peeters, and R. Burke Chem. Eng. Sc. $20,529(1965)$.

(b) T.I. Cottrel, in The Strength of Chemical Bonds, Butterworths, London 1958, p.270.

(c) J.A. Kerr, Chem. Rev. 66, 465 (1966).

222(a) M. Ruchardt, Ber. 24, 2599 (1961).

(b)M. Buchardt and R. Hecht, Ber. 28, 2460 (1965).

223. C.J. Collins, W. T. Rainey, W.B. Smlth, and I.A. Kaye, J. Am. Chem. Soc. 81, 460 (1959).

224: R.0. Kan, in Organic Photochemistry, McGraw-H111, New York, N.Y. 1966, P.196.

225. L.F. Fleser, Experiments in Organic Chemistry, D.C. Heath and Co., Boston, 1957, p.103.

226. E. Wedekind and E. Frohlich, Ber. 40, 1009 (1907). 227. O.C. Dermer and J. King, J. Am. Chem. Soc. 63, 3232
(igh2).

228. S. G. Smith, A.H. Fainbers and S. Winstein, J. Am. Chem. Soc. 83, 618 (1961).

229. J.A. Riddick, E. E. Toops, Jr., in Organic Solvents Technique of Organic Chemistry, edit. A. Weissberger, Interscience Pub., John Wiley and Sons, Inc., New York, N.. Y. Vo1. ?, 1955, p.297.

230. E. M. Kosower, J. Am. Chem. Soc. 80, 3253 (1958).

231. W. Oroshik, G: Karmans, and A.D. Mebane, J. Am. Chem. Soc. 24, 295 (1952).

232(a)M. Kasha, J. Opt. Soc. 38, 929 (1948).

(b)c.s. Ch1ld, J. Opt. Soc. 51, 895 (1961).

(c) G. Schwarzenbach and. K. Lutz, Helv. Chim. Acta 23, 1139 (1940).

233. C.R. Masson, V. Boekelhe1de, and W.A. Noyes, Jr., Photochemical Reactions in Technique of Organic Chemistry, edit. A. Welssberger, Vol. 2 , Interscience Pub., John Wiley and Sons, New York, N.Y. 1956, p.282. 
234. J.G. Calvert and J.N. P1tts, Jr., Photochemistry, John Wiley and Sons, Inc., New York, N.Y., 1966, p.783.

235. A. Beckett and G. Porter, Trans. Faraday Soc. 52, 2038 (1963).

236. I.F. Fleser, J. Am. Chem. Soc. 46, 2639 (1924).

237. C.A. Bunton, D.R. Llewellyn, and G. Stadman, J. Chem. Soc. 568 (1958).

238. I. Dostrovsky and D. Samuel, Inorgan1c Isotope Symthes1s, edit. R.H. Herber, W. Benjamin, New York, N.Y. 1962, p.134.

239. C.K. Rollefson and C.F. Oldershaw, J. Am. Chem. Soc. 54, 977 (1932).

240. A.C. Hutenburg, J. Halperin, and H. Taube, J. Am. Chem. Soc. 73,4487 (1951).

241. R.L. Shriner, R.C. Fuson, and D.Y. Curtin, The Systematic Identification of Organic Compounds, 4 th edit., John W1ley and Sons, Inc., New York, N.Y., 1957, p.254.

242. C.A. Parker, Proc. Roy. Soc. (Iondon) A220, 104 (1953).

243. J.N. P1tts, J.D. Margerum, B.P. Taylor, and W. Brim, J. Am. Chem. Soc. 27, 5499 (1955).

244. G.S. Forbes and L.J. He1dt, J. Am. Chem. Soc. 56, 2363 (1934).

245(a)F.S. Johnson and K. Watanabe, R. Tousry, J. Opt. Soc. Am. 41, 402 (1951).

(b) R. Allison, J. Bums, and J. Tuzzolino, J. Opt. Soc. Am. 54,747 (1964).

(c)N. Kristianpoller, J. Opt. Soc. Am. 54, 1285 (1964).

(d)R. Allison, J. Burns, and J. Tuzzolino, J. Opt. Soc. Am. 54, 1381 (1964).

(e)N. Kristianpoller and R.A. Knapp, Appl. Opt1cs, 2, 915 (1964).

(f)R.A. Knapp, Appl. Opt1cs, 2, 1334 (1963).

(8) R.A. Knapp and A.M. Smith, Appl. Opt1cs, 2, 637 (1964). 